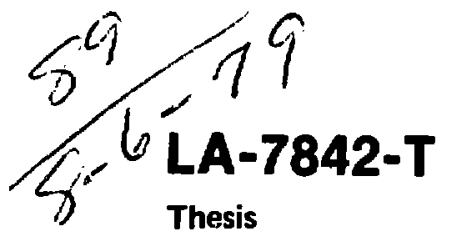

The Plasma Boundary Lajer and Magnetopause Layer of the Earth's Magnetosphere

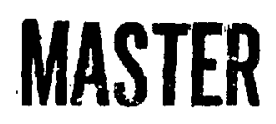

응

¿

齐

:

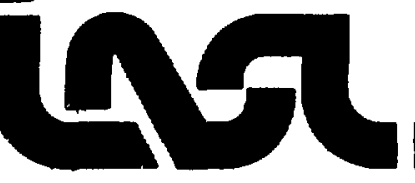




\title{
The Plasma Boundary Layer and Magnetopause Layer of the Earth's Magnetosphere
}

\author{
Timothy Eric Eastman*
}

"EG\&G, Inc., P.O. Box 809, Lo: Alamos, NM 87544.
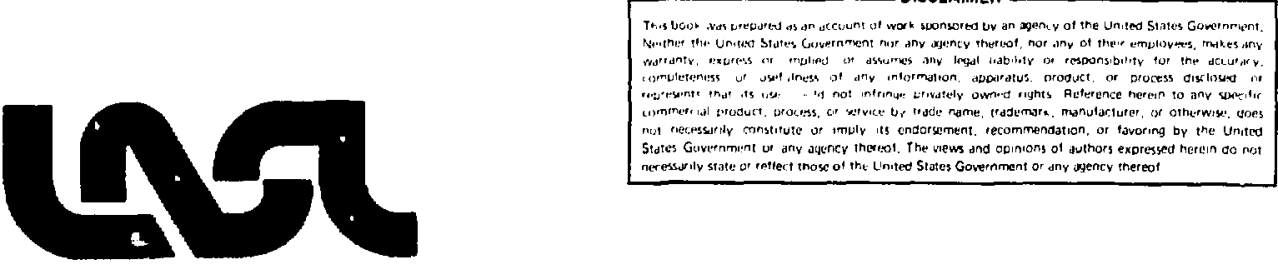
PREFACE

The principal objective of this thesis is the identification of the primary source of plasma for the plasma boundary layer (PBL) of the earth's magnetosphere. This objective will be pursued by a detailed study of IMP 6 observations of the PBL and by a comparison of these observations with various theories of the solar wind-magnetosphere interaction. This study has been motivated by several factors.

- No coordinated study of magnetic field and plasma parameters were reported for the low-latitude sunward PBL until Eastman et al. (1976)

- The first two basic problems posed for the international magnetospheric study (IMS) cannot be answered without a detailed knowledge of PBL characteristics. These problems are the "effect of the interplanetary magnetic field on topography, topology and stability of the magnetospheric boundary," and "the mechanisms for the entry of solar wind plasma into the magnetosphere" (Roederer, 1977).

- The magnetosphere is a unique laboratory for plasma physics. In particular, the PBL is a transition region in a medium- $\beta$, collisionless plasma in which the ion-to-electron temperature ratio is much greater than one. The parameter regime observed in the $\mathrm{PBL}$ is significant for current research in controlled thermonuclear fusion. As shown by recent ISEE dual satellite results (Paschmann et al., 1978), there are significant space and time variations of the magnetopause layer (MPL) and PBL. Movements of the MPL often exceed the spacecraft speed, typically about $2 \mathrm{~km} / \mathrm{s}$ near the MPL. Thus, a study of the MPL and PBL from a single satellite cannot fully separate space and time 
variations. To offset these limitations, this study, with its description of the MPL and PBL, is based on over 100 IMP 6 crossings of the magnetosphere's sunward surface and more than 100 additional crossings of the tajlward extension of these bo:andary regions.

The people who have contributed in some way to my thesis are too numerous to list. I am deeply grateful to Edward W. Hones for consenting to advise me in this work, for his steady support, and for providing an outstanding example of the work of a careful and systematic physicist. Thanks are due to S. J. Bame and coworkers of the Los Alamos Scientific Laboratory (LASL) Group P-4 for making available their outstanding IMP 6 plasma data set, and to N. F. Ness and D. H. Fairfield of Goddard Space Flight Center (NASA/GSFC) for IMP 6 magnetometer data, and to C.-I. Meng for providing energetic electron data obtained from the Unive:sity of California, Berkeley, energetic particle detector on the IMP 6 satellite. I gratefully acknowledge the support of LASL, P Division, and particularly J. Conner for providing support for attending some meetings that were crucial for my work and also for providing various materials and office space. Thanks are due to the Los Alamos office of EG\&G, Inc. for their cooperation during the four years that I worked on this program. Financial assistance that helped make this work possiole was provided by the EG\&G Employee Educational Training Assistance Program, supported by the US Department of Energy. Some of the people to whom I am grateful for helpful suggestions and criticism are Dan Baker, Don Baker, Alex Bratenahl, Nancy Crooker, Don Fairfield, Terry Forbes, S. Peter Gary, Walter Heikkila, Paul Higbie, Joe Kan, Joseph Lemaire, Don Lemons, C.-I. Meng, A. Nishida, Götz Paschmann, Karl 
Schindler, Bengt Sonnerup, G. S. (Dyke) Stiles, Vytenis Vasyliunas, and J. David Winningham. Thanks are due to several more members of Group P-4 at LASL including Earl Tech for data base management and software assistance, Jean DeField for typing, and Rose Watts for computer assistance. I am especially grateful to the University of Alaska Geophysical Institute and the National Science Foundation for sponsoring the Exteralal Degree Program which made this work possible while I continued my full-time work at EG\&G, Inc. This program provides an outstanding opportunity for employees who cannot leave their work for various reasons to fulfill a residency requirement. The present work is submitted in hopes that this excellent program can be continued and can afford similar opportunities to other people working in the geophysical science and related fields. This work was supported by the US Department of Energy. 
CONTENTS

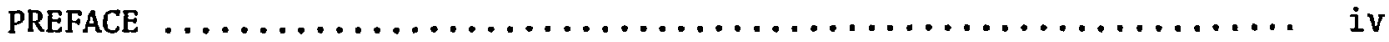

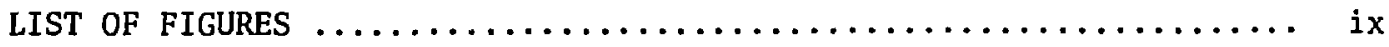

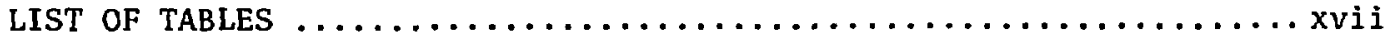

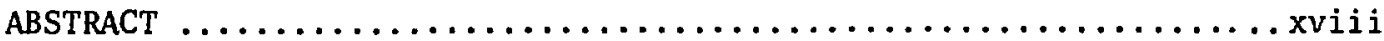

CHAPTER 1: INTRODUCTION

1.1 Scientific Objective and General Considerations ......... 1

1.2 Historical Notes $\ldots \ldots \ldots \ldots \ldots \ldots \ldots \ldots \ldots \ldots \ldots \ldots \ldots \ldots$

1.3 Selection Criteria and Observational Limitations ....... 6 CHAPTER 2: OBSERVATIONS

2.1 General Considerations $\ldots \ldots \ldots \ldots \ldots \ldots \ldots \ldots \ldots \ldots \ldots \ldots \ldots$

2.2 IMP 6 Trajectory and Instrumentation $\ldots \ldots \ldots \ldots \ldots \ldots \ldots$

2.3 Case-by-Case Study $\ldots \ldots \ldots \ldots \ldots \ldots \ldots \ldots \ldots \ldots \ldots \ldots \ldots \ldots \ldots$

2.3.1 Basic Data Set ........................ 14

2.3.2 20 June 1972 IMP 6 Crossing ................. 16

2.3 .31 May 1973 IMP 6 Crossing .................. 29

2.3.4 16 June 1973 IMP 6 Crossing ................ 38

2.3.5 14 April 1972 IMP 6 Crossing ................ 44

2.3.6 22 May 1972 IMP 6 Crossing ............... 52

2.3.7 4 March 1972 IMP 6 Crossing ................ 60

2.3 .8 ISEE Crossings $\ldots \ldots \ldots \ldots \ldots \ldots \ldots \ldots \ldots \ldots \ldots \ldots \ldots \ldots . \ldots \ldots$

2.4 Statistical study $\ldots \ldots \ldots \ldots \ldots \ldots \ldots \ldots \ldots \ldots \ldots \ldots \ldots \ldots \ldots$

2.4.1 Basic Questions $\ldots \ldots \ldots \ldots \ldots \ldots \ldots \ldots \ldots \ldots \ldots \ldots$ 
2.4 .2 Density Variations $\ldots \ldots \ldots \ldots \ldots \ldots \ldots \ldots \ldots \ldots \ldots$

2.4.3 Bulk Plasma Fl ow Characteristics ............. 79

2.4.4 PBL-MPL Thickness Ratio .................. 81

2.4.5 PBL Thickness Estimates $\ldots \ldots \ldots \ldots \ldots \ldots \ldots \ldots \ldots \ldots$

2.4.6 Energetic Electron Characteristics ............. 90

2.5 Laboratory Experiments $\ldots \ldots \ldots \ldots \ldots \ldots \ldots \ldots \ldots \ldots \ldots \ldots \ldots . \ldots 2$

2.6 Review of Observations $\ldots \ldots \ldots \ldots \ldots \ldots \ldots \ldots \ldots \ldots \ldots$

CHAPTER 3: THEORY AND OBSERVATIONS

3.1 Physical Characteristics of the MPL and PBL .......... 97

3.1.1 Plasma Characteristics ................. 97

3.1 .2 MHD Discontinuities .................... 101

3.1 .3 Comparisons of Spectra ................... 104

3.1.4 Particle and Energy Flux in the PBL ........... 107

3.2 Observations and Theory $\ldots \ldots \ldots \ldots \ldots \ldots \ldots \ldots \ldots \ldots \ldots$

3.2.1 Early Wodels .......................... 109

3.2 .2 Recent Models ........................ 116

3.3 Diffusion Due to Plasma Kinetic Instabilities .......... 124

3.4 Review of Theory and Observations $\ldots \ldots \ldots \ldots \ldots \ldots \ldots \ldots 131$

CHAPTER $4:$ CONCLUSIONS $\ldots \ldots \ldots \ldots \ldots \ldots \ldots \ldots \ldots \ldots \ldots \ldots \ldots \ldots \ldots \ldots$

APPENDIX A IMP 6 Instrumentation and Analysis Techniques ....... 136

A1 Electrostatic Analyzer $\ldots \ldots \ldots \ldots \ldots \ldots \ldots \ldots \ldots \ldots \ldots \ldots$

A2 Analysi, Techniques $\ldots \ldots \ldots \ldots \ldots \ldots \ldots \ldots \ldots \ldots \ldots \ldots \ldots \ldots$

APPENDIX B Supplementary IMP 6 MPL-PBL Crossings $\ldots \ldots \ldots \ldots \ldots \ldots \ldots$

APPENDIt $C$ Minimum Variance Analysis $\ldots \ldots \ldots \ldots \ldots \ldots \ldots \ldots \ldots$

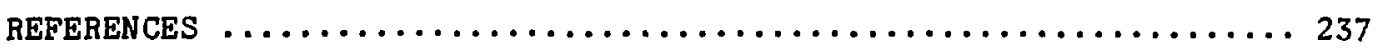




\section{LIST OF FIGURES}

1.1 A sketch of the earth's magnetosphere showing the plasma boundary layer (PBL) and magnetopause layer (MPL)........ 2

2.1.1 Two sample IMP 6 orbits plotted in solar ecliptic (SE)

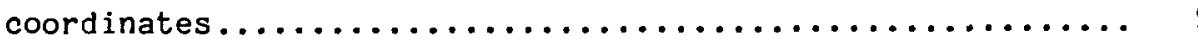

2.2.2 Satellite coverage of the sunward magnetopause and plasma boundary layer (PBL) for IMP 6, HEOS 2 and the ISEE 1 and ISEE 2 satellite pair. ISEE coverage is given only for

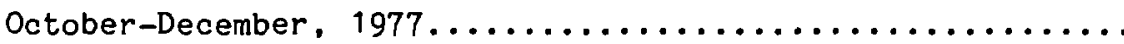

2.2.3 The locations, in geocentric solar magnetospheric (GSM) coordinates, of IMP 6 magnetopause crossings selected for high time resolution analysis. Crossings that showed distinct changes in electron density or spectra $(x)$ at the MPL are distinguished from crossings that showed no distinct changes (filled circle or square). Rapid crossings had a crossing time of 30 seconds to three minutes and either showed a distinct density change $(x)$ or no distinct density change ( ). Eight crossings (o) did not have a clear magnetopause signature.

2.3 .2 .120 June 1972 piasma data..................... 17

2.3.2.2 20 June 1972 plasma and $\hat{i} i e l d$ data................. 18

2.3.2.3 20 June 1972 plasma flow and field projections plotted from the magnetosheath (MS) to the magnetosphere(MG) earthward of the MPL-PBL region......................... 21

2.3.2.4 Magnetic field hodograms, field magnitude and electron density for the 20 June 1972 crossing of the MPL......... 22 
2.3.2.5 Azimuthal averages of the ion distribution function plotted for six to ten minute intervals for the 20 June 1972

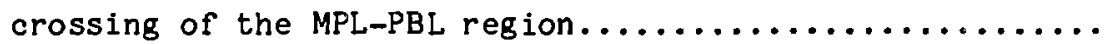

2.3.2.6 Azimuthal averages of the distribution function plotted for ^100 s intervals for the 20 June 1972 crossing........... 25

2.3.2.7 Electron differential spectra plotted four times evory $\sim 12.5 \mathrm{~s}$ at successive locations near the MPL for the 20 June 1972 crossing. Nearby magnetospheric spectra are also plotted for comparison....................

2.3 .3 .11 May 1973 plasma data...................... 30

2.3.3.2 1 May 1973 plasma and field data................. 31

2.3.3.3 1 May 1973 plasma flow and field projections.......... 32

2.3.3.4 Energetic electron (47- to 350-keV) pitch-angle distributions for the 1 May 1973 crossing.......................... 33

2.3.3.5 Magnetic field hodograms, field magnitude and electron density for the 1 May 1973 crossing of the MPL......... 34

2.3.3.6 Electron differential spectra plotted four times every $\$ 12.5 \mathrm{~s}$ at successive locations near the MPL for the 1 May 1973 crossing. Nearby magnetospheric spectra are also plotted for comparison...................... 36

2.3.3.7 Azimuthal averages of the distribution function plotted for s100 s intervals for the 1 May 1973 crossing............ 37

2.3.4.1 16 June 1973 plasma data........................ 39

2.3.4.2 16 June 1973 plasma and field data................ 40 2.3.4.3 16 June 1973 plasma flow and field projections......... 41 
2.3.4.4 Energetic electron (47- to $350-\mathrm{keV}$ ) pitch-angle distribu-

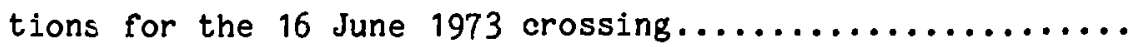

2.3.4.5 Azimuthal averages of the distribution function plotted for $\backsim 100 \mathrm{~s}$ intervals for the 16 June 1973 crossing......... 43

2.3.5.1 14 April 1972 plasma data..................... 45

2.3.5.2 14 April 1972 plasma and field data................ 46

2.3.5.3 14 April 1972 plasma flow and field projections......... 47

2.3.5.4 Magnetic field hodograms, field magnitude and electron density for the 14 April 1972 crossing of the MPL......... 48

2.3.5.5 Azimuthal averages of the distribution function plotted for five to seven minute intervals for the 14 April 1972

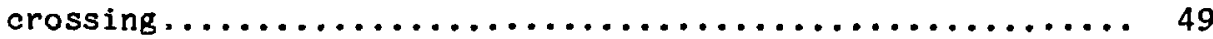

2.3.5.6 Electron differential spectra plotted four times every $\sim 12.5 \mathrm{~s}$ at successive locations near the MPL for the 14 April 1972 crossing. Nearby magnetospheric spectra are

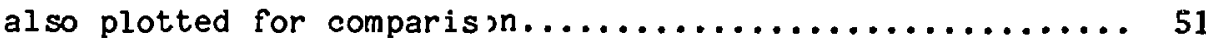

2.3 .6 .122 May 1972 plasma data...................... 53

2.3.6.2 22 May 1972 plasma and field data................. 54

2.3.6.3 22 May 1972 plasma flow and field projections........... 55

2.3.6.4 Magnetic field hodograms, field magnitude and electron density for the 22 May 1972 crossing of the MPL......... 56

2.3.6.5 Azimuthal averages of the distribution function plotted for $\checkmark 100 \mathrm{~s}$ intervals for the 22 May 1972 crossing 
2.3.6.6 Electron differential spectra plotted four times every $\backsim 12.5 \mathrm{~s}$ at successive locations near the MPL for the 22 May 1972 crossing. Nearby magnetospheric spectra are also plotted for comparison...................... 59

2.3.7.1 4 March 1972 plasma data....................... 61

2.3.7.2 4 March 1972 plasma and field data................ 62

2.3.7.3 4 March 1972 plasma flow and field projections.......... 63

2.3.7.4 Magnetic field hodograms, field magnitude and electron density for the 4 March 1972 crossing of the MPL......... 64

2.3.7.5 Energetic electron (47- to 350-keV) pitch-angle distributions for the 4 March 1972 crossing................. 65

2.3.7.6 Electron differential spectra plotted four times every $12.5 \mathrm{~s}$ at successive locations near the MPL for the 4 March 1972 crossing. Nearby magnetospheric spectra are also plotted for comparison.....................

2.3.7.7 Azimuthal averages of the distribution function plotted for s100 s intervals for the 4 March 1972 crossing.......... 68

2.3.8.1 Two- and three-dimensional plasma and field parameters for the ISEE 1 crossing of 3 November 1977. Solar magnetospheric (GSM) coordinates are used for spacecraft positions with $R$ (in $R_{E}$ ), local time ( $L T$ in hours), and latitude (in degrees). Solid lines denote protons and dotted lines denote electrons in the plots of density ( $\mathrm{N}$ in $\mathrm{cm}^{-3}$ ) and temperature ( $T$ in degrees Kelvin). Bulk flow directions are given in terms of azimuth $\left(\Phi_{p}\right)$ and elevation $\left(\Lambda_{p}\right)$ in spacecraft coordinates (close to solar ecliptic 
coordinates) based on both the 2D (solid line) and 3D (dots) moment analysis. $M, E, N$, or $S$ denote morning, evening, northward, or southward flow components, respectively. Total plasma pressure (solid line) and magnetic field pressure (dotted line) are given in units of $10^{-8}$ dynes $/ \mathrm{cm}^{2}$ (left scale). The dotted curve also gives the magnetic field strength (in gammas) by using the quadratic righthand scale. The magnetopause is marked by a solid vertical line and the inner surface of the plasma boundary layer is marked by a dashed line. Magnetosheath plasma is sampled before 0751 UT and magnetospheric plasma is sampled after

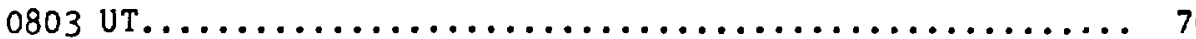

2.3.8.2 Two- and three-dimensional plasma and field parameters for the INEE 2 crossing of 8 November 1977. This figure uses the sam? format as Figure 2.3.8.1 except that an isolated p] asma boundary layer is marked of $f$ by vertical dashed lines at 0254:50 and 0258 UT. The magnetopause layer is centered on the vertical solid line at 0252 UT......... 72

2.4.2.1 Electron density data are plotted for selected PBL crossings characterized by a single, well-defined MP:... Satellite time in the PBL is denoted by $T_{B L}$. Data points that give averages of three-second electron density samples are separated by $\simeq 12.5 \mathrm{~s}$ time intervals unless noted by $\triangle T$. The $P B L$ is given the same apparent width in all plots with the MPL positioned on the right hand side. All locations are given in GSM coordinates............. 76 
2.4.2.2 Electron density values sampled in the magnetosheath adjacent to the MPL-PBL regions shown in Figure 2.4.2.1. Density values are plotted every $\backsim 12.5 \mathrm{~s}$ (i.e., averaged over four electron spectra sampled during each spin period) at equal time spacing for all crossings............ 78

2.4.3 Summary of plasma flow in the sunward plasma boundary layer as observed by IMP 6, HEOS 2, ISEE 1 and ISEE 2. Approximate projections of observed flow vectors are given on the noon-midnight meridian and equatorial plane cross-sections of the PBL based on satellite crossings with an earth centered angle of $<15^{\circ}$ from the $X-Z$ and $X-Y$ planes, respectively. Flow vectors marked between the two crosssections are observed PBL flow vectors projected onto the magnetopause surface. The magnetosheath bulk flow adjacent to the magnetopause was assumed to be parallel to the magnetopause surface for this plot. This drawing has been made to scale except that the PBL is enlarged $n 50 \% . . \ldots \ldots$

2.4.5.1 Plasma boundary layer thickness values are plotted versus the local $z$ component of the magnetic field, $B_{z}$, as measured by IMP 6 in the magnetosheath adjacent to the magnetopause. From the larger set of 90 crossings, only those crossings which permitted a rough estimate of $B_{z}$ were used................................... 
2.4.5.2 Statistics of nominal PBL thickness, $L_{\mathrm{PBL}}$ (a) and comparisons of $\mathrm{L}_{\mathrm{PBL}}$ with are length along the magnetopause measured from the subsolar point (b), IMF $B_{z}(c), \operatorname{IMF} B_{y}$ (d) and $X_{G S M}(e) . L_{P B L}$ peaks at $S=7$ vo $8 R_{E}$ (plot b) due to enhanced $\mathrm{PBL}$ thickness in the cusp regions............. 87

2.4.5.3 Nominal PBL thicknesses plotted on the sunward magnetopause surface. All results are based on IMP 6 crossings except for four points near local noon below $\lambda_{\mathrm{GSM}}=30^{\circ}$ which are

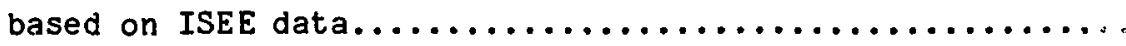

2.5 Convection pattern in the magnetospheric equatorial plane

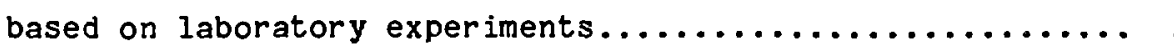

3.2.1.1 Plasma characteristics in the Ferraro (a) and Parker (b) magnetopause models...........................

3.2.1.3 Magnetospheric convection pattern produced by a viscous

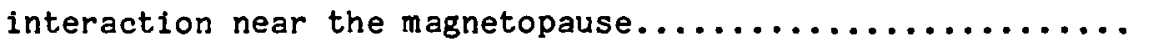

3.2.1.4 Plasma flow and field configuration in the Dungey recon-

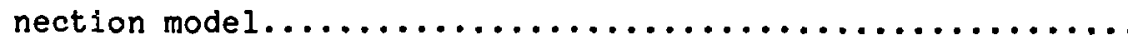

3.2.1.5 Calculated plasma and field parameters (solid lines) based on a reconnection model (Yang and Sonnerup, 1977) compared with observed parameters (dashed lines) for the 14 April 1972 IMP 6 crossing of the MPL-PBL region. Angles subtended relative to the subsolar point are plotted along the abscissa. The PBL thickness has been plotted to fit between the intermediate wave (IW) or magnetopause and the earthward edge of the slow expansion fan (SEF) 
3.2.2.1 Comparison of expected (a) and observed (b) proffles of mean energy per particle...................... 118

3.2.2.2 The impulsive penetration model using an equatorial crosssection of the magnetosphere.

A1 Errors due to counting statistics as a function of density. $N$,density: $E$, mean thermal energy: $u$, energy density: $V$, velocity magnitude; AN, anisotropy magnitude; $\phi_{v}$, velocity angle; $\phi_{A N}$, anisotropy angle (angles are read on the right scale)

A2 Variations of plasma parameters with total energy. The velocity distribution is assumed isotropic in the rest frame of the moving plasma which has a speed of $100 \mathrm{~km} / \mathrm{s} . .143$

A3 Variations of plasma parameters with the polar angle of the flow velocity.......................... 144

C1 The coordinate system obtained by the minimum variance

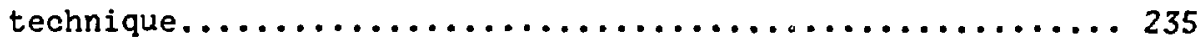




\section{LIST OF TABLES}

2. 3 Selected IMP 6 Crossings of the MPL-PBL Region.......... 15

2.4.4 PBL versus MPL Thickness..................... 82

2.4.5 Nominal Plasma Boundary Layer Thisknness Values and their Means verus $\mathrm{x}_{\mathrm{GSM}} \ldots \ldots \ldots \ldots \ldots \ldots \ldots \ldots \ldots \ldots \ldots \ldots \ldots \ldots$

3.1.1 Observed MPL and PBL Plasma Parameters............... 98

3.1.2 Classification of Shock Waves and Discontinuities........ 103

3.1.4 Ion Plasma Parameters for a Typical Dawn-Dusk Keridian

Cross-Section of the PBL which is Separated into Four

Zones to Illustrate Typical Variations within It........ 107

A1 Electrostatic Analyzer Parameters.................. 137

A2 Energy Levels Sampled by the LASL Electrostatic Analyzer... 137 
THE PLASMA BOUNDARY LAYER AND MAGNETOPAUSE LAYER

OF THE EARTH'S MAGNETOSPHERE

by

Timothy Eric Eastman

\begin{abstract}
IMP 6 observations of the plasma boundary layer (PBL) and magnetopause layer (MPL) of the earth's magnetosphere indicate that plasma in the low-latitude portion of the $P B L$ is supplied primarily by direct transport of magnetosheath plasma across the MPL and that this transport process is relatively widespread over the entire sunward magnetospheric boundary. The region of antisunward flowing solar wind plasma located downstream from the earth's bow shock is called the magnetosheath. Near the transition between the magnetosheath and the magnetosphere is a region of magnetosheath-like plasma called the PBL which is located on field lines with a magnetospheric orientation. I define the MPL as the current layer (separating the magnetosheath from the $P B L$ ) through which the magnetic field shifts in direction. High temporal resolution (3-s average) data reveal that in a majority of IMP 6 magnetopause crossings, no distinct changes in electron density or energy spectra are observed at the MPL. In all IMP 6 crossings, some magnetosheath-like plasma is observed earthward of the MPL, which implies the existence of the PBL. PBL electron energy specta are often virtually indistinguishable from the adjacent magnetosheath spectra. Bulk plasma flow in the low-latitude part of the PBL as observed by IMP 6 almost always has an antisunward component and often has a significant cross-field component. This region is located in a quasi-trapping zone that contains predominantly closed field lines. The PBL thickness is highly variable and, in general, is much larger than the MPL thickness. A brief analysis of the lower hybrid drift instability indicates that it can provide substantial cross-field diffusion and, thus, contribute to the PBL population. Observations suggest that significant plasma energy dissipation occurs in the low latitude portion of the PBL. A ermparison of various theoretical descriptions of the solar wind-magnetosphere interaction illustrates the problems posed for all theoretical models. The most significant problems are the high spatial and temporal variations of plasma and field parameters observed near the magnetopause. Realistic models of the magnetopause interaction must (1) account for the presence of the PBL and (2) account for transport of magnetosheath plasma across the MPL.
\end{abstract}


CHAPTER 1

INTRODUCTION

\subsection{Scientific Objective and General Considerations}

The principal objective of this work is to identify the primary source for the plasma boundary layer (PBL) of the magnetosphere by a detailed study of IMP 6 data collected in this boundary region and by a comparison of these observations with various theories of the solar wind-magnetosphere interaction. As shown in Figure 1.1, the PBL separates the magnetosheath, composed of solar wind plasma that has become more turbulent downstream oi the bow shock, from the magnetospheric plasma in which particle motion is controlled mainly by the geomagnetic field. Many confusing labels have been applied to the PBL because of a wide variety of spacecraft trajectories, data sets, and time resolution. Here $\mathrm{PBL}$ is intended as a generic term (having a neutral connotation) for the layer of magnetosheath-like plasma observed earthward of the magnetopause layer (MPL) everywhere near the magnetospheric boundary. Other terms applied to the PBL include plasma mantle for the highlatitude part, magnetotail boundary layer for the tailward part, and low-latitude boundary layer for the low-latitude region. Magnetospheric boundary layer is another generic term for the PBL. MPL denotes the current layer (separating the magnetosheath from th. PBL) through which the magnetic field shifts in direction. The current layer is often resolved by IMP 6 with both the plasma and magnetic field data so that MPL is more descriptive because of the limited denotation of "pause" as a bounding surface. 


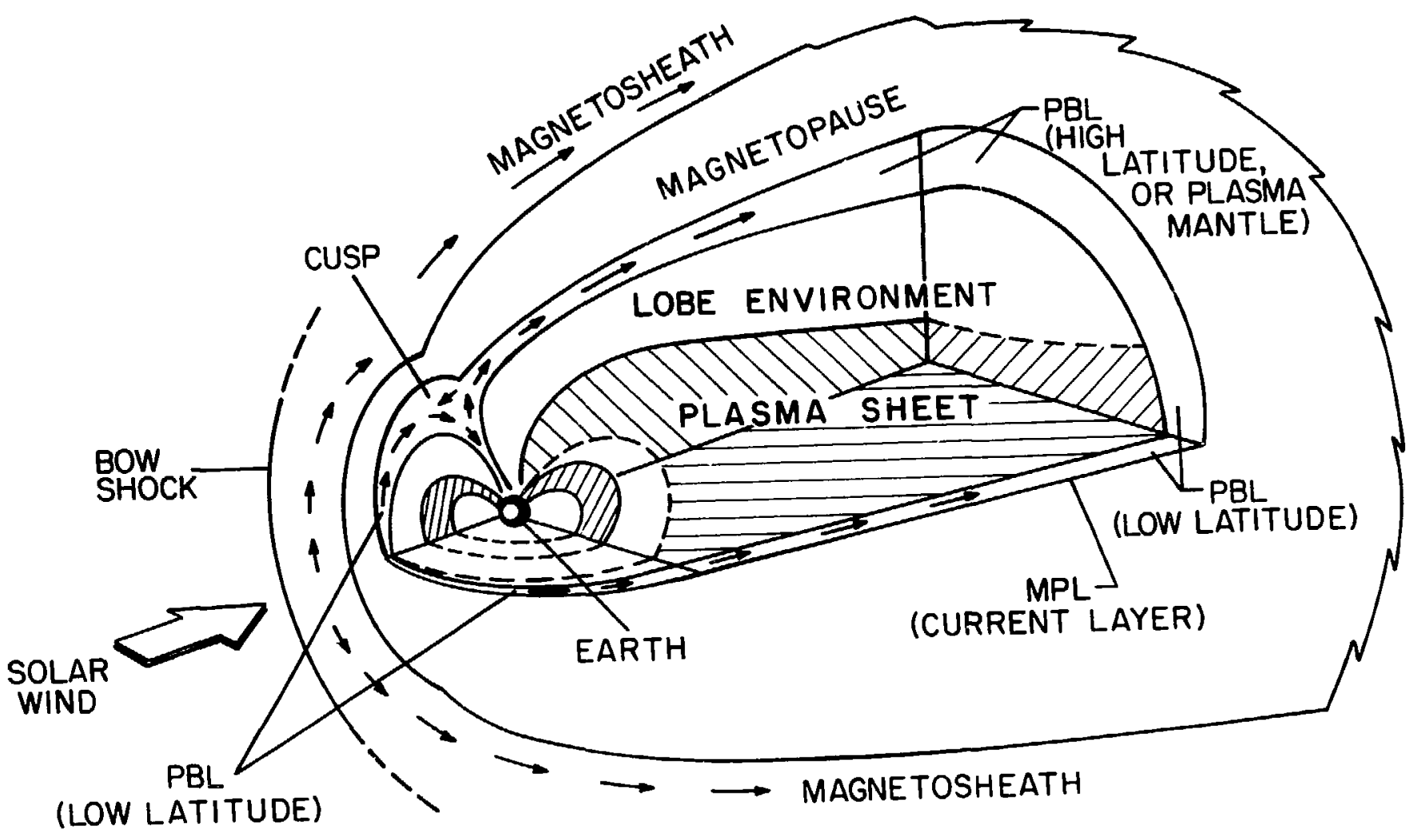

Figure 1.1 A sketch of the earth's magnetosphere showing the plasma boundary layer (PBL) and magnetopause layer (MPL). 
Any mass, momentum or energy transfer from the solar wind to the magnetosphere is accomplished by way of the MPL and PBL. The plasma sheet and cusp regions are in some way supplied by the PBL, and these regions in turn provide the source of plasma precipitation in the auroral ionosphere. To identify the primary source of plasma for the PBL a detailed description of MPL and PBL characteristics is given to provide an observational framework for improved theoretical descriptions. At present, no adequate theoretical description exists for solar wind-magnetosphere interaction processes; magnetotail dynamics studies are limiter by this lack of knowledge.

\subsection{Historical Notes}

Solar plasma was treated first as a neutral ionized stream by Lindemann (1919) and Chapman (1923) who discussed the effect of polarization charges established as this stream impacts the geomagnetic field. Chapran and Ferraro (1932) first noted that solar wind plasma would confine the geomagnetic field on the sunward side without substantial penetration beyond a current layer. During the first half of this century, this confinement of the geomagnetic field was considered an intermittent phenomenon limited to magnetic storm periods. It was not recognized until the discovery of the solar wind (Biermann, 1951) that the magnetosphere is present all the time. Although theoretical treatments of a current layer near the magnetospheric boundary were made as early as 1952 by Ferraro (1952), in situ observations had to wait until the adrent of satellite probes that could intersect the magnetopause.

Cahill and Amazeen (1963) reported the first clear identification of the sunward magnetopause made by a magnetometer on board the Explorer 12 
satellite. An uncertain magnetopause location was noted on at least one pass of IMP 1 "because the magnitude and direction of the magnetic field changed at different locations" (Hess, 1968). Earlier, Wolfe et al. (1966) noted a confusion from differences between magnetic and plasma magnetospheric boundaries. Freeman et al. (1968) made a more extensive description based on an unusual set of magnetopause crossings by the ATS 1 synchronous satellite and stated that "a new component of ion flow was found immediately inside the magnetospheric boundary. This flow had characteristics nearly identical to the magnetosheath ion flow; the flow was parallel to the boundary in the downstream direction, and the total ion flux was nearly the same as that found across the boundary in the magnetosheath." They considered this magnetosheath-like flow to be the convective return flow envisioned by Axford and Hines (1961) in their closed magnetospheric model (see section 3.2.1). The first identification and extensive discussion of plasma data of the PBL was made by Hones et al. (1972) based on Vela satellite observations along the flanks of the magnetotail. They called the observed transition region from the magnetosheath to the magnetosphere the "magnetotail boundary layer." Akasofu et al. (1973) extended this study of Vela data showing the PBL and included examples of crossings of both the high-latitude portion of the PBL bordering the tail lobe environment or extended polar caps region (the high-latitude boundary layer (HLBL) or plasma mantle) and the low-latitude boundary layer (LLBL) bordering the plasma sheet or outer ring current regions. Plasma and field measurements made by the HEOS 2 satellite made possible the first detailed description of the PBL in the polar magnetosphere. Rosenbauer et al. (1975) related their 
observations of the high-latitude portion of the $\mathrm{PBL}$, which they termed the plasma mantle (or plasma coat), to the Vela observations although most of the Vela crossings were made through the LLBL. Fc lowing Hones et al. (1972), and Akasofu et al. (1973), Rosenbauer et al. (1975) stated that "the most probable cause for the formation of the mantle is the dayside merging of terrestrial and interplanetary field lines." The first detailed specification of LLBL characteristics was provided by Eastinain et al. (1976) based on corre?ated plasma and field observations by IMP 6 . These data indicated that magnetosheath plasma could cross the MPL and contribute to the PBL plasma. Eastman et al. (1976) suggested an intermixing of magnetosheath and magnetospheric plasma in the PBL, which has been supported recently by ISEE ion measurements (Haerendal et al., 1978b). In the past few years an extensive set of PBL observations has been reported based on HEOS 2 (Paschmann et al., 1976; Haerendel et al., 1978a), Explorer 33 (Crooker, 1978), IMP 6 (Eastman and Hones, 1978; Eastman and Hones, 1979), IMP 7 (Scarf et al., 1977) and ISEE data (Paschmann et al., 1978; Russell and Elphic, 1978). These observations have established the generally continuous presence of the PBL over the entire magnetospheric surface. 


\subsection{Selection Criteria and Observational Limitations}

This thesis will provide a detailed study of more than 30 IMP 6 crossings of the MPL and PBL selected from more than 100 sunward crossings and more than 100 tailward crossings. (Most of the selected cases are sunward crossings.) Selection criteria were availability of generally complete plasma and magnetic field data, and a minimum number of magnetopause crossings. Crossings near the cusp region and near the noon meridian were also favored in this selection. For as many cases as possible, energetic electron (47- to $350-\mathrm{keV}$ ) data wore obtained for evaluation of pitch-angle distributions. Multiple magnetopause crossings are evident in a few of the crossings; however, the selected cases clearly show the principal characteristics of the MPL and PBL. They also show significant differences in detailed structure which is complicated by the presence of significant spatial and/or temporal variations. A single satellite such as IMP 6 cannot separate directly space and time variations. Multiple magnetopause crossings may be caused by waves propagating along the magnetopause boundary. Local magnetopause speeds are known to range to $>30 \mathrm{~km} / \mathrm{s}$ (Russell and Elphic, 1978; Williams, 1978), so that the IMP 6 satellite, traveling about 2 $\mathrm{km} / \mathrm{s}$ near the magnetopause, can be overtaken several times by the current layer during a single pass. Although multiple MPL crossings are common, multiple sunward MPL-PBL crossings are relatively rare in the IMP 6 data set. Throughout this work, possible implications of large spatial and temporal variations should be kept in mind. 


\section{CHAPTER 2}

\section{OBSERVATIONS}

\section{1 General Considerations}

In general, an experimental study of the magnetopause region is a case-by-case study or a statistical study. High spatial and temporal variations near the magnetopause 1 imit the value of case-by-case studies; however, they are required for detailed information about physical characteristics of the boundary regions. Because of the observed complexity of these regions, this thesis provides both a case-by-case and a statistical study including correlated plasma and field observations. Sections 2.3 and 2.4 present a case-by-case and statistical study, respectively, of the MPL and PBi. This chapter is then followed by a review of the implications of the IMP 6 observations for various theoretical descriptions of the solar wind-magnetosphere interaction.

Observations of changes in plasma and field characteristics across the PBL and MPL are crucial to determining the source of PBL plasma. The magnetopause region is a low density $\left(<50 \mathrm{~cm}^{-3}\right)$, collisionless, medium- $P$ plasma. $B$ is the ratio of plasma to magnetic field energy density. Values of magnetic induction near the MPL range from 10 to 35 $r\left(1 \gamma=10^{-5}\right.$ gauss $)$ and a typical ion cyclotron radius there is about $100 \mathrm{~km}$. The magnetosheath exhibits solar wind bulk flow characteristics, whereas particle motion within the magnetosphere is controlled mainly by the geomagnetic field. The MPL and PBL represent transition regions between the magnetosheath and the magnetospheric plasma regimes. 


\subsection{IMP 6 Trajectory and Instrumentation}

IMP 6 (alternate names are Explorer 43 and IMP I) was launched into a highly elliptic orbit (apogee $\approx 33 R_{E}$, perigee $\leqslant 21 / 2 R_{E}$, and inclination range of 28.8 to $38^{\circ}$ ) on March 13,1971 . The satellite was spin stabilized with the spin axis pointing toward the south ecliptic pole. Figure 2.2.1 from Fairfield et al. (1973) shows solar ecliptic $X-Y$ and $X-Z$ projections of two orbits which illustrate gradual orbital changes that take place in an inertial coordinate system. The orbital period is $\simeq 4.1$ days and two magnetopause crossings occur during each orbit except when the apogee is deep inside the magnetotail. As the orbit precesses, the satellite returns in one year to sample equivalent positions on the magnetopause. Consequently, the IMP 6 trajectory eventually provided magnetopause crossings over the entire sunward hemisphere at low latitudes as shown in Figure 2.2.2 (which uses GSM coordinates**). The subsolar region is deleted because, for the few crossings made near the subsolar point, the plasma data were gathered in a mode not used for this study. Fairfield (1976) has described

* The solar ecliptic coordinate system (SE) is earth-centered with the $\mathrm{X}$-axis along the earth-sun direction. The $\mathrm{Z}$-axis is taken perpendicular to the ecliptic plane and is positive toward the north ecliptic pole. A right-hand system then defines the Y-axis which is directed duskward.

* The geocentric solar magnetospheric coordinate system (GSM) is earthcentered with the $X$-axis directed along the earth-sun direction. However, the $Z$-axis is now taken perperdicular to the $X$-axis in the plane defined by the $X$-axis and the earth's dipole direction. With a righthand system, the $\mathrm{Y}$-axis is again directed duskward. 

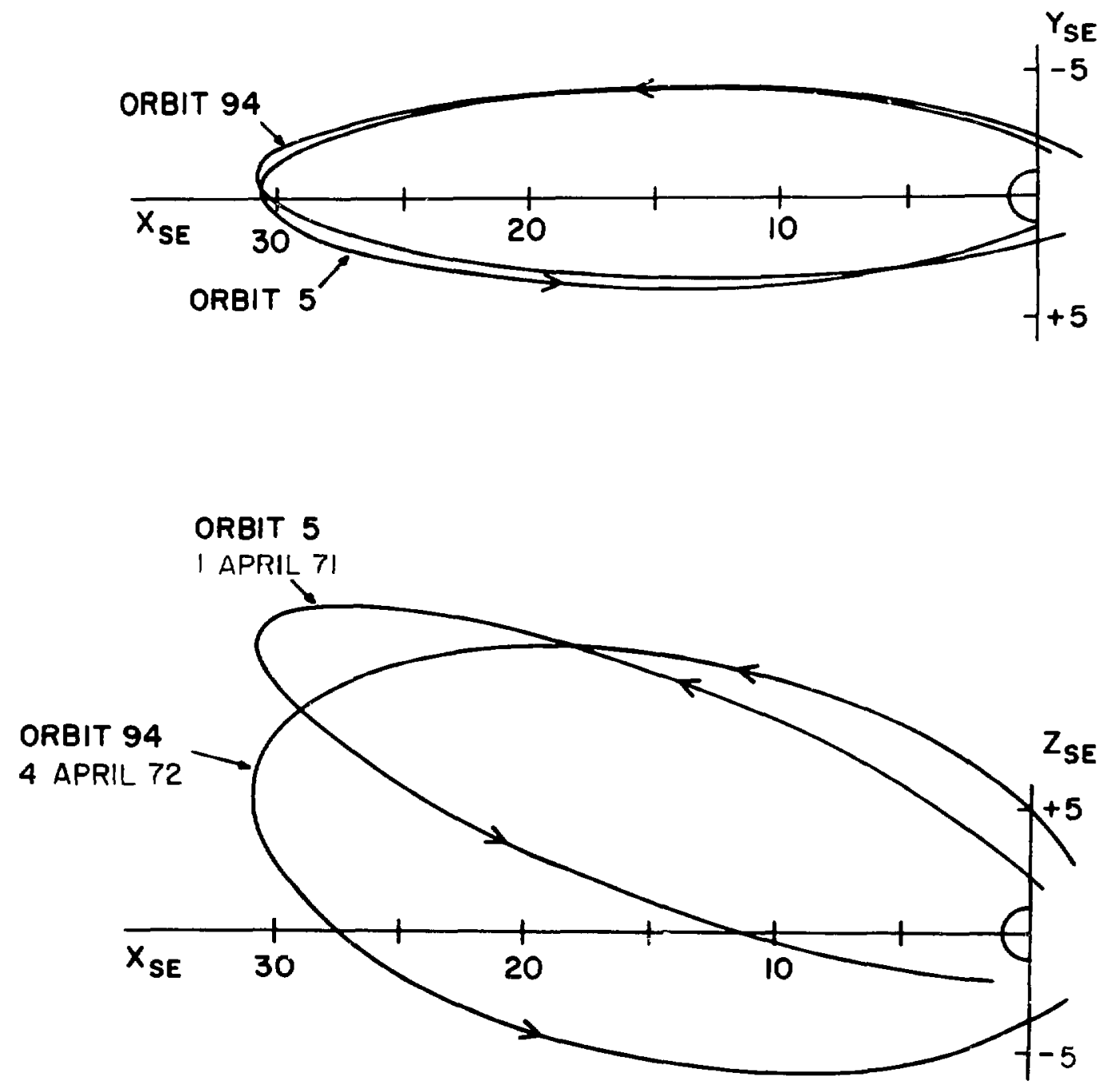

Figure 2.1.1 Two sample IMP 6 orbits plotted in solar ecliptis (SE) coordinates. 
magnetometer data for a few crossings in the subsolar region. The IMP 6 satellite continued to supply data until 2 October 1974.

Figure 2.2.2 shows the range of magnetopause crossing locations for IMP 6 as well as for HEOS 2 and the ISEE satellites. IMP 6 and HEOS 2 have complementary trajectories that result in nearly total coverage of the sunward magnetospheric boundary (c.f., Sckopke and Paschmann, 1978). ISEE 1 and ISEE 2 orbits have a smaller inclination $\left(\simeq 27^{\circ}\right)$ than the IMP 6 orbits and they penetrate the MPL and PBL at lower latitudes.

Selected IMP 6 MPL-PBL crossings for this study are presented in Figure 2.2.3 in GSM coordinates. The coverage is complete except for the subsolar region and low latitudes along the dawn side. A few crossings of the cusp region were made by IMP 6 and these are discussed in detail by Fairfield and Hones (1978).

The Los Alamos Scientific Laboratory (LASL) electrostatic plasma analyzer on board IMP 6 sampled ions in the energy range $140 \mathrm{eV}$ to 28.8 $\mathrm{keV}$ and electrons in the range $13.3 \mathrm{eV}$ to $18.1 \mathrm{keV}$. Ion and electron distributions were sampled with a three-degree-wide analyzer fan with a polar acceptance range of $\pm 45^{\circ}$ centered about the ecliptic plane. Plasma spectra were sampled every $3 \mathrm{~s}$ and a rough (four-angle) two-dimensional velocity distribution of both ions and electrons was obtained for each spacecraft rotation period (s $12.5 \mathrm{~s}$ ). Complete 32-angle, 16-energy velocity distributions of ions and electrons were obtained approximately every $100 \mathrm{~s}$. Our study also includes magnetic field data from the Goddard Space Flight Center (NASA/GSFC) magnetometer (courtesy of D. H. Fairfield) and energetic electron data obtained by 


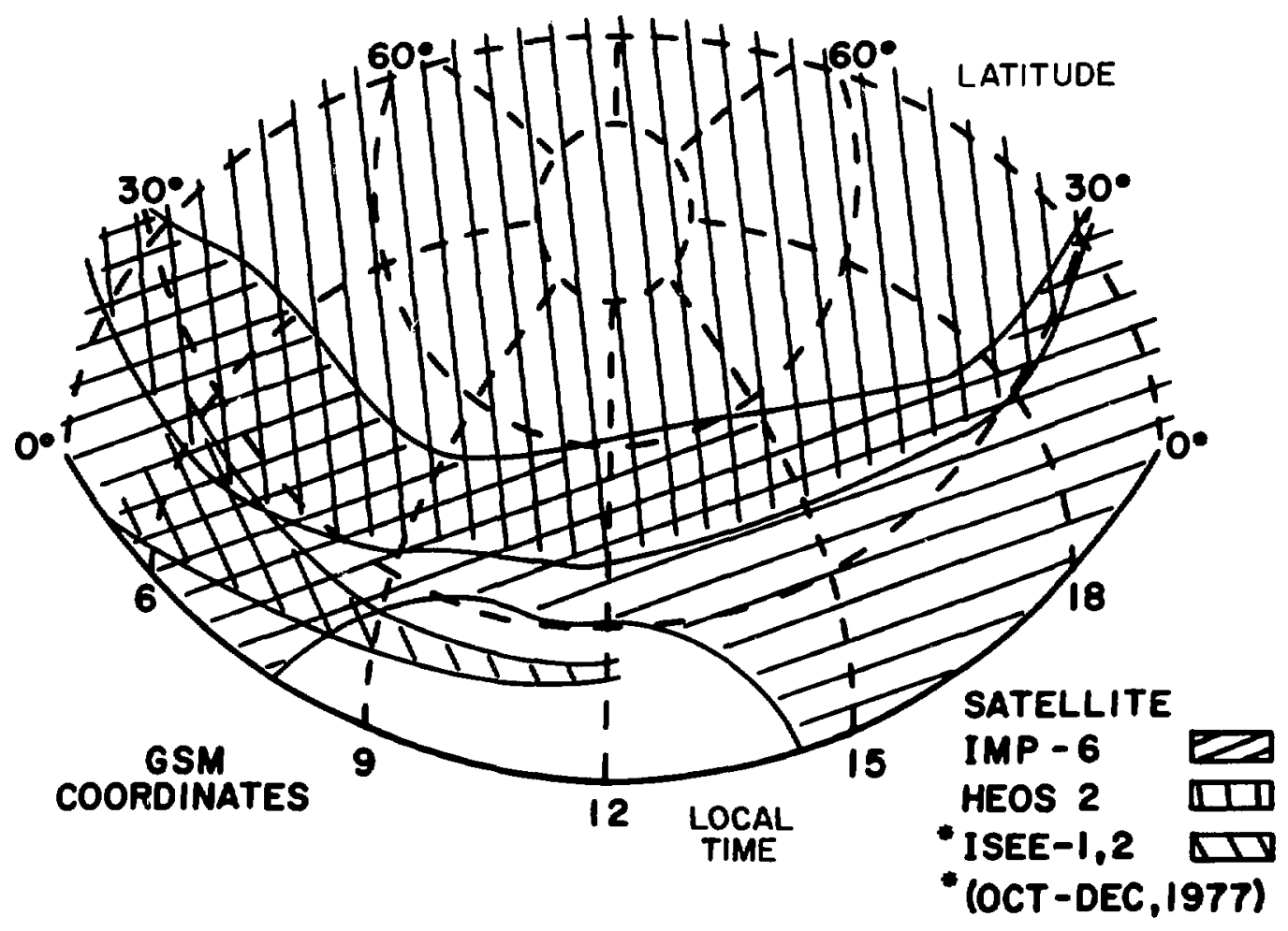

Figure 2.2.2 Satellite coverage of the sunward magnetopause and plasma boundary layer (PBL) for IMP 6, HEOS 2 and the ISEE 1 and ISEE 2 satellite pair. ISEE coverage is given only for October-December, 1977. 


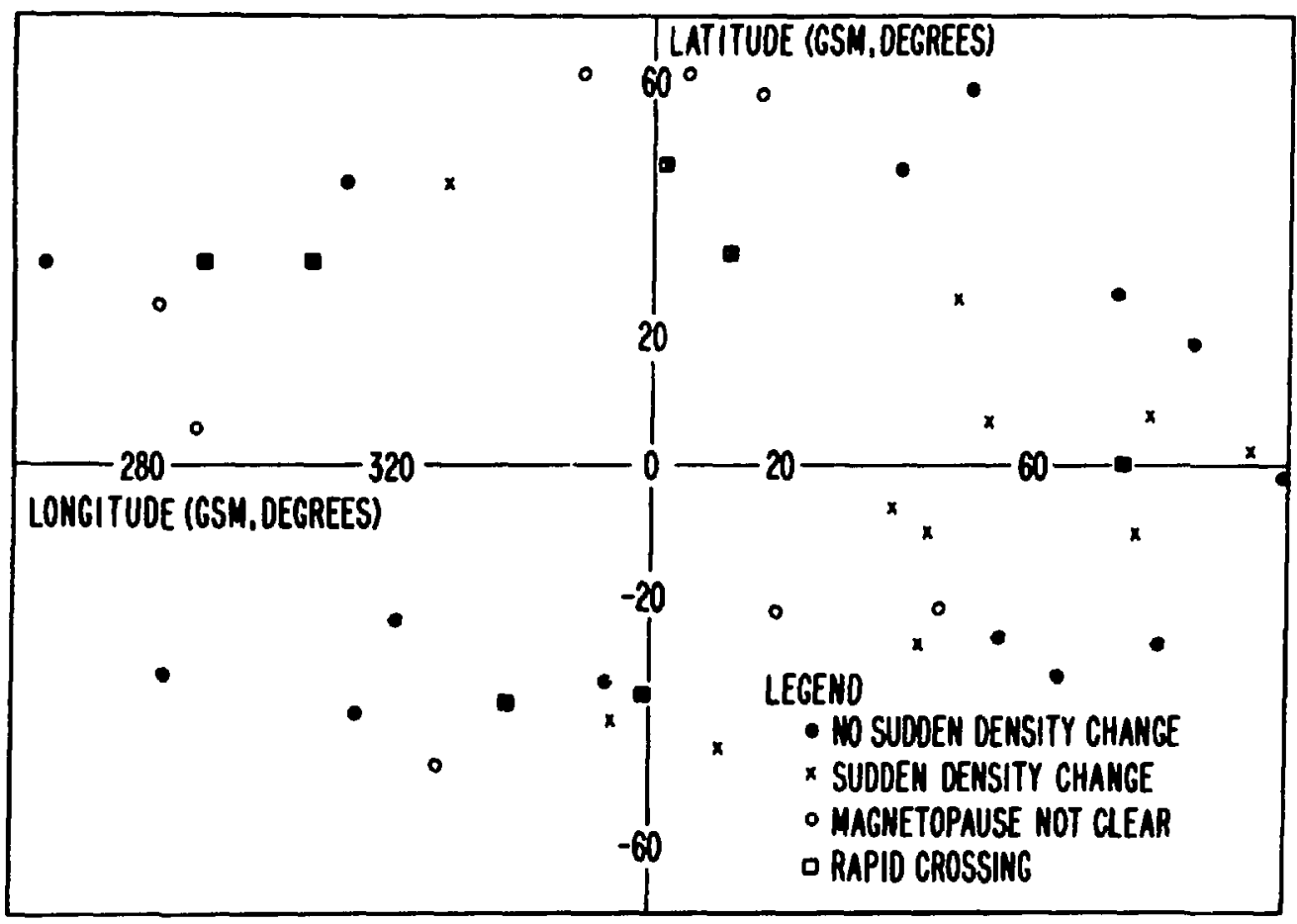

Firure 2.2.3 The locations, in geocentric solar magnetospheric (GSM) soordinates, of IMP 6 magnetopause crossings selected for high time resolution analysis. Crossings that showed distinct changes in eiectron density or spectra $(x)$ at the MPL are distinguished from crossings that showed no distinct changes (filled circle or square). Rapid crossings had 3 crossing time of 30 seconds to three minutes and either showed a distinct density change $(x)$ or no distinct density change ( $\bullet$. Eight crossings (o) did not have a clear magnetopause signature. 
solid-state detectors (courtesy of C.-I. Meng, University of California, Berkeley).

A detailed description of IMP 6 analysis techniques and instrumentation is provided in Appendix A. The format for plasma and field data presentation is thoroughly described for the first example given in section 2.3 . 


\subsection{Case-by-Case Study}

\subsubsection{Basic Data Set}

Selected IMP 6 MPL-PBL crossings included in this thesis are given in Table 2.3. Locations of these cases are marked in Figure 2.2.3. Values of $\mathrm{K}_{\mathrm{p}}$ and $\mathrm{B}_{\mathrm{z}}$ (King, i977) for the same hour and the previous hour, respectively, are also provided for reference. The $\mathrm{K}$-index is a measure (on a logarithmic scale) of fluctuations in magnetic activity from 0 to 9. $K_{p}$ is the worldwide weighted average of the $K$-index. $B_{z}$ denotes an hourly average of the Z-component of the interplanetary magnetic field.

A detailed description is provided for several MPL-PBL crossings that exhibit the principal features of these boundary regions. Data for the remaining crossings listed in Table 2.3 are presented in Appendix $B$. Two ISEE satellite crossings are presented in section 2.3 .8 for comparison with the IMP 6 data. 
Table 2.3. Selected IMP 6 Crossings of the MPL and PBL

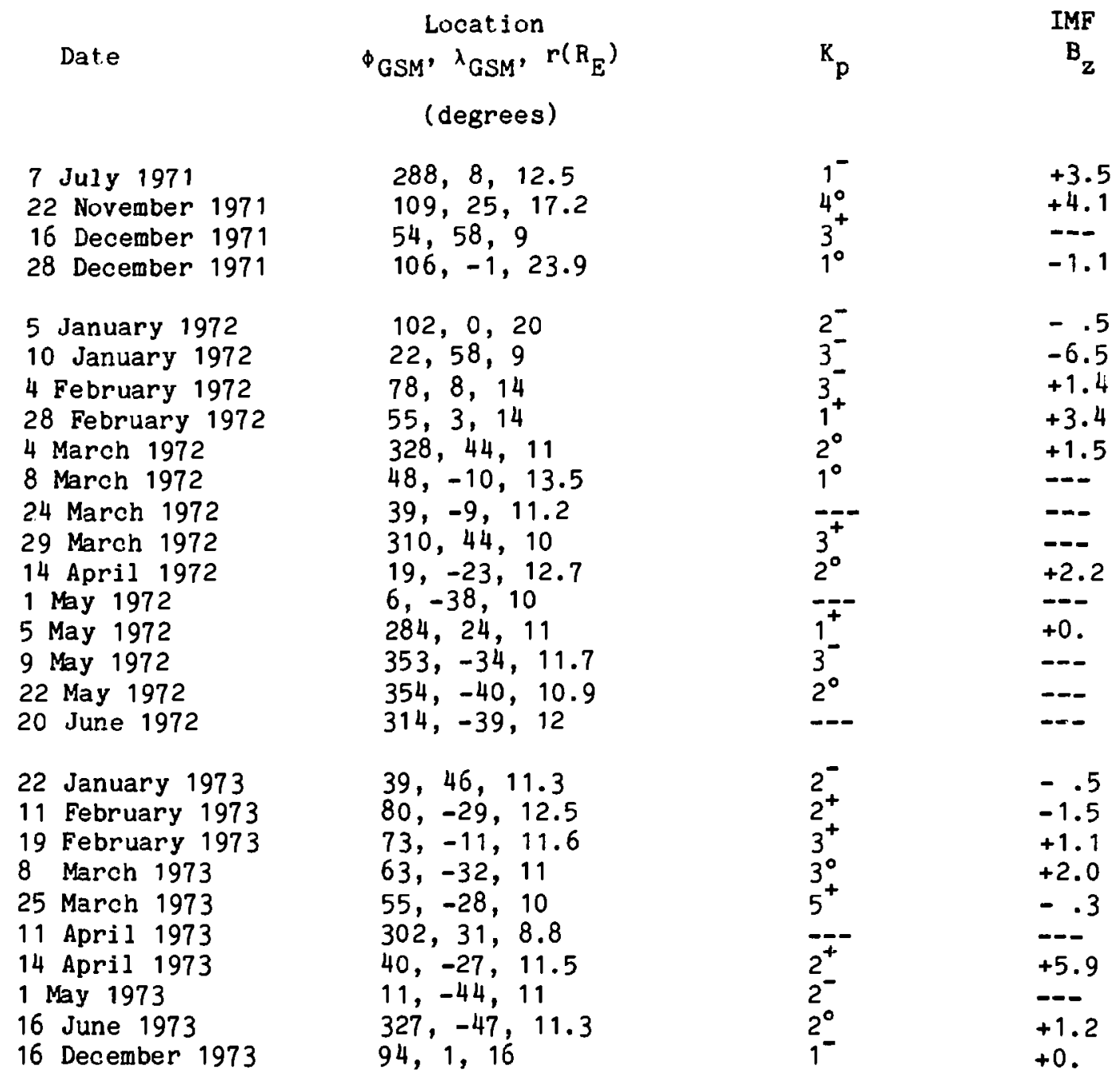




\subsubsection{June 1972 IMP 6 Crossing}

This dawn side crossing, shown in Figures 2.3.2.1 and 2.3.2.2, illustrates most of the characteristic MPL-PBL features. Near 0210 UT the magnetic field makes a clear transition from a southward directed magnetosheath field to a northward directed magnetospheric field. This transition in direction marks the magnetopause layer (MPL) or current layer (marked by a pair of solid vartical lines in the figures). In this case, a generally duskward directed magnetopause current is required to accompany the field direction change. It is significant that field magnitude or field fluctuations as well as the plasma parameters do not distinctly mark the MPL. Instead, the MPL is a region of finite thickness within which the change in magnetic field direction is accomplished.

The plasma boundary layer ( $\mathrm{PBL}$ ) is distinguished as the region of antisunward plasma flow located earthward of the MPL. Typically, the overall density and bulk flow velocity decrease from the MPL to the inner surface of the PBL (left to right in Figures 2.3.2.1 and 2.3.2.2) is accompanied by an increase in mean thermal energy and continued magnetosheath-level fluctuations of the magnetic field, given by the standard deviation $\mathrm{SD}_{\mathrm{B}}$ of field magnitude values. Some crossings, as in this case, show a continued increase of thermal energy for a brief interval earthward of the PBL. The plasma $B$ (see bottom of Figure 2.3.2.2 for plots of energy density) usually drops to $\leqslant 1$ in the inner part of the PBL consistent with the decay of field fluctuations. This is because, for low $B$, the magnetic field is dominant and is not readily perturbed by the plasma. In the plots of plasma energy density, the 


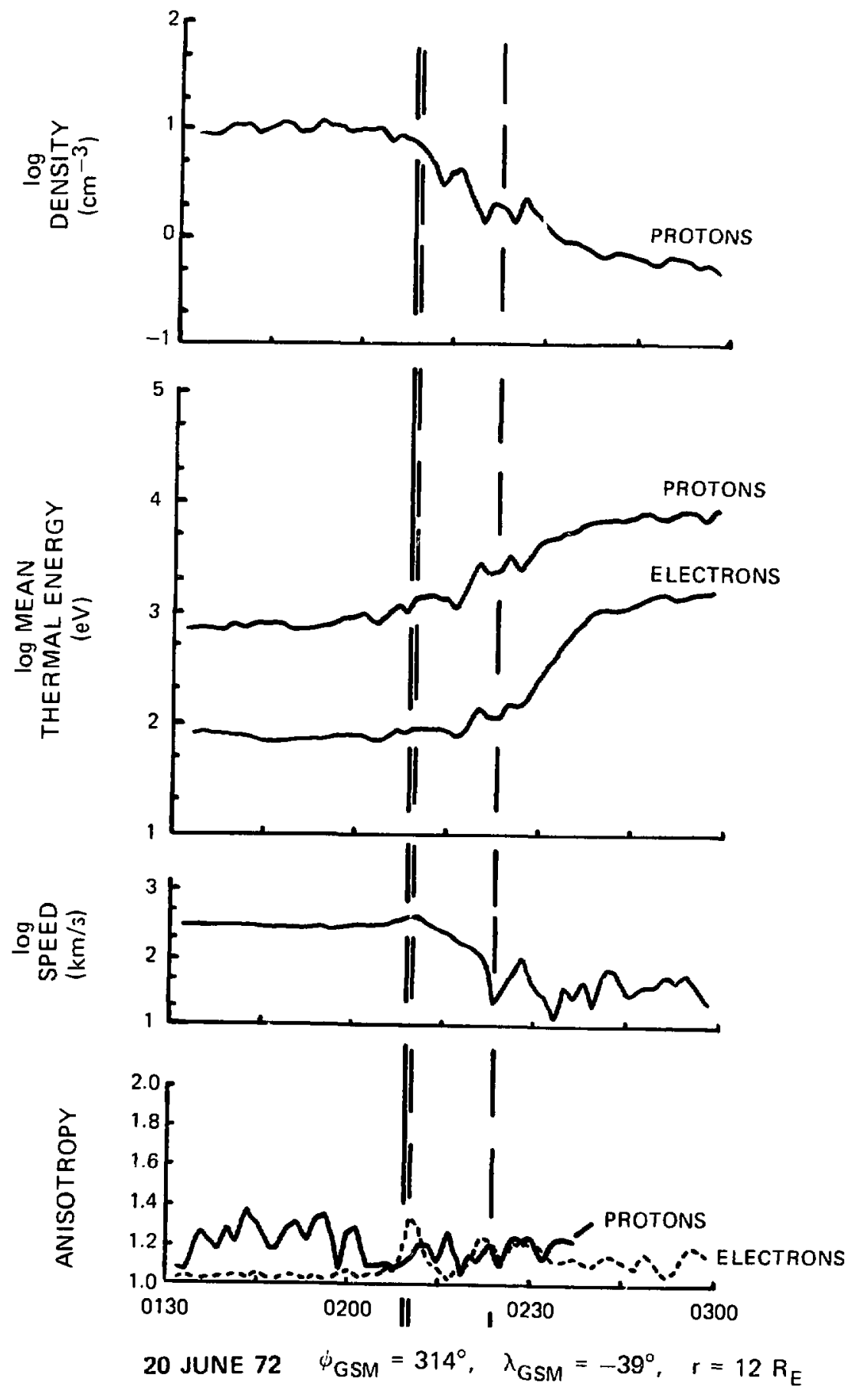

Figure 2.3.2.1 20 June 1972 plasma data. 


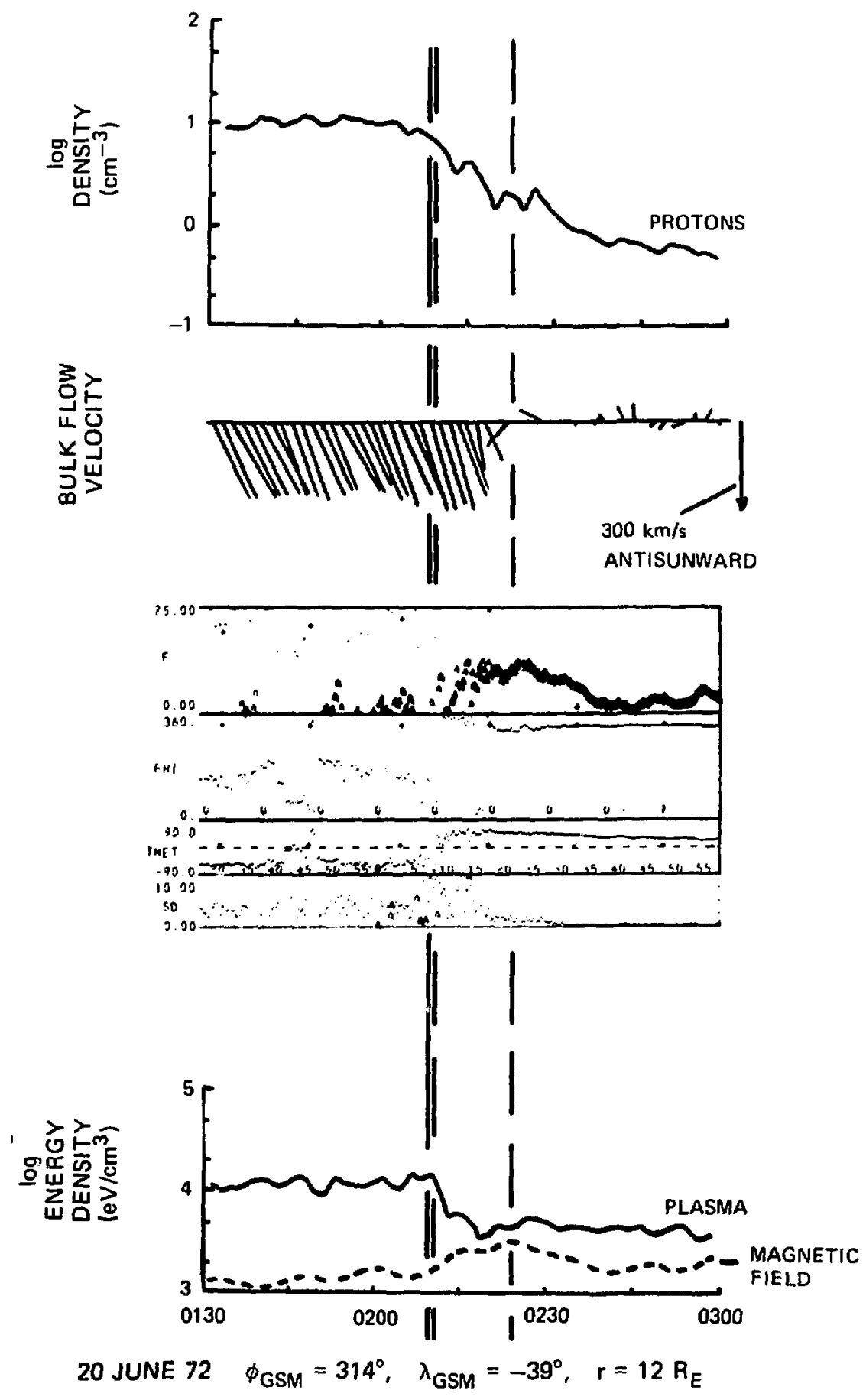

Figure 2.3.2.2 20 June 1972 plasma and field data. 
kinetic energy density is not included so that plasma $B$ can be estimated directly from the curves for plasma and field energy density. Within the PBL the plasma flow directions are more variable and usually shift into a direction (for the ecliptic plane flow component) that is farther from the $\mathrm{X}_{\mathrm{GSM}}$-axis than the direction of the nearby magnetosheath flow. This flow shift characteristic is much more clearly illustrated in other crossings to be discussed (e.g., the 1 May 1973 crossing, Figure 2.3.3.3). A slight enhancement in speed occurs near the MPL in this crossing (an increase of $\backsim 100 \mathrm{kw} / \mathrm{s}$ relative to the nearby magnetosheath flow).

The data for each IMP 6 PBL-MPL crossing are presented as pairs of correlated plots similar to Figures 2.3.2.1 and 2.3.2.2. Density plots are repeated at the top of each graph for comparison. Magnetic field parameters are presented in solar ecliptic (SE) coordinates unless otherwise noted as GSM coordinates in the figure. These plots include magnetic field magnitude (F) in $\gamma\left(=10^{-5}\right.$ gauss) (dots are $0-25 \gamma$, A for 25-50 $\gamma$, B for 50-75 $\gamma$, etc.), longitude (PHI), latitude (THET) and standard deviation of the magnitude (SD) (dots are 0-10 $\gamma$, A for 10-20 $\gamma$, etc.). Values of magnetic field parameters are $15.36 \mathrm{~s}$ averages of 80-ms time-interval data values. Positions marked by $a+$ in the F, PHI, and THET plots represent nominal dipole field values. Pressure anisotropy is calculated from the sampled velocity distribution as the ratio of maximum pressure to the pressure evaluated for the direction perpendicular to the maximum pressure axis. In the PBL and nearby magnetosheath the anisotropy direction for ions is, in general, perpendicular 
to the magnetic field so that $P_{1}>P_{\|}$(see Crooker et al., 1978). Electrons often have no significant anisotropy. Occasionally, the electron anisotropy becomes significant near the PBL, although the anisotropy direction relative to the magnetic field varies from srossing to crossing.

Many crossings show a significant cross-field bulk flow component in the PBL. For the 20 June 1972 crossing, simultaneous bulk flow and magnetic field vectors are plotted in Figure 2.3.2.3 which shows the solar ecliptic $Y-X$ and $Y-Z$ projections of the magnetic field vectors. (Axes are illustrated at the right-hand side of Figure 2.3.2.3.) On the $Y-X$ plot, bulk flow velocities are also given by vectors with arrowheads. Although the MPL is clearly defined, the flow vectors show no distinct change until the inner part of the PBL is reached. In the PBL, the angle between the flow vectors (effectively sampled in an ecliptic plane projection by the electrostatic analyzer) and the field vectors range from $\backsim 145$ to $\sim 175^{\circ}$ with an estimated angular error for the flow vectors of $\leqslant 5^{\circ}$ before 0217 UT. Flow vectors shown are 100-s averages and the field vectors are 1.25-s averages. Error estimates for plasma and field parameters are discussed in Appendix A.

Detailed characteristics of magnetic field changes through the MPL can provide information on the type of discontinuity represented ty the current layer (see section 3.1.2). A magnetic field hodogram that shows such detailed field changes is given in Figure 2.3.2.4 based on a minimum variance calculation (Sonnerup and Cahill, 1967). This technique assumes that the magnetic field component perpendicular to the layer remains constant during the satellite crossing. The minimum variance 


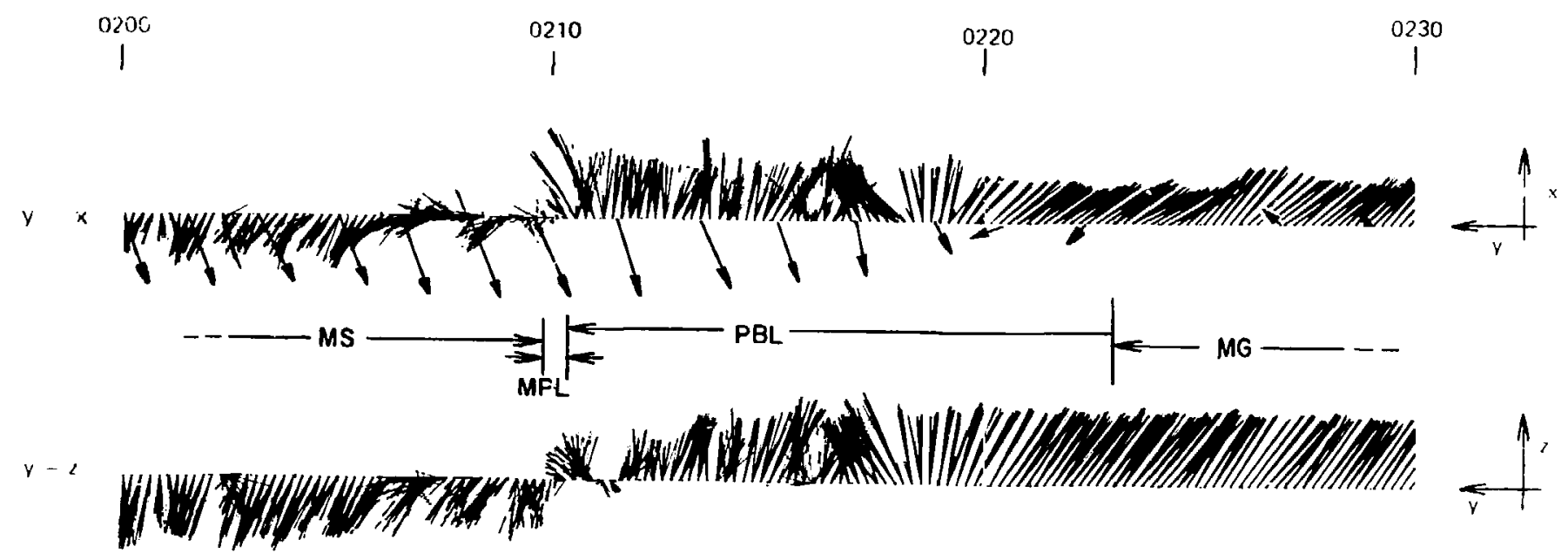

Figure 2.3.2.3 20 June 1972 plasma flow and field projections plotted from the magnetosheath (MS) to the magnetosphere (MG) earthward of the MPL-PBL region. 

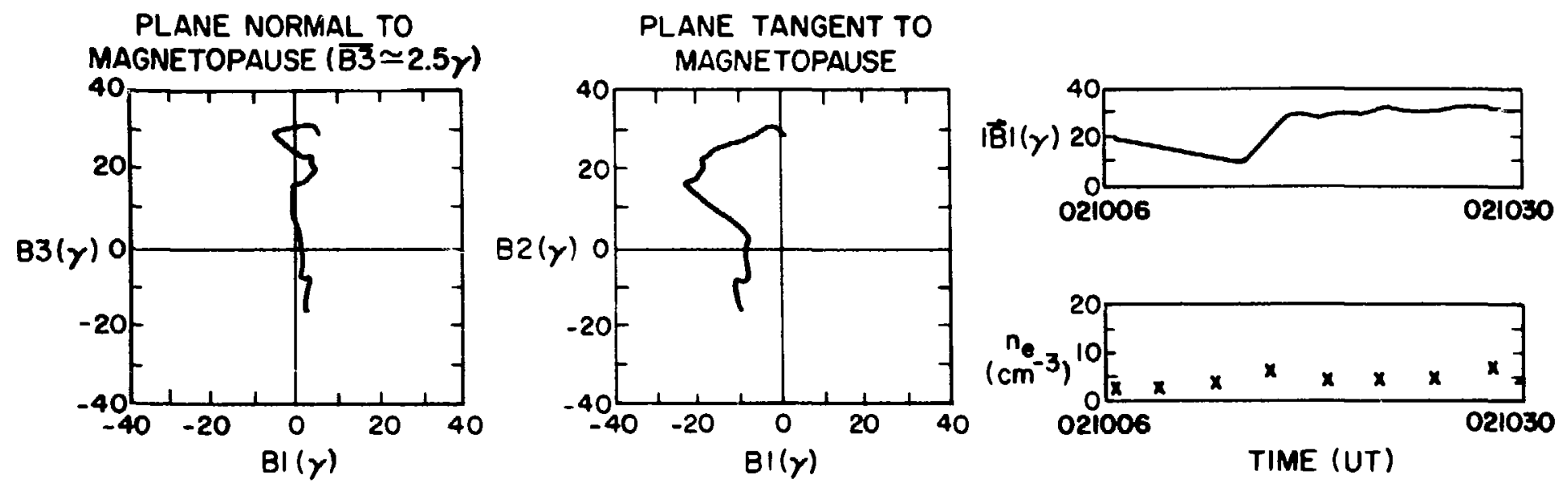

Figure 2.3.2.4 Magnetic field hodograms, field magnitude and electron density for the 20 June 1972 crossing of the MPL. 
analysis method is described in Appendix $C$. Magnetic field hodograms are computed in a local coordinate system in which B3 denotes the direction of minimum $\vec{B}$ (magnetic field) variance (an outward magnetopause normal direction). B1 denotes the direction of maximum $\mathrm{B}$ variance (enerally lirected northward along the sunward magnetopause) and B2 completes a right-hand coordinate system (thus, in general, B2 is directed westward along the sunward magnetopause).

The B3-B1 hodogram represents a view of the tip of the magnetic field vector as it moves in a plane positioned perpendicular to the current layer with $B 1$ directed roughly northward and B3 directed along the outward normal. The normal component of the magnetic field $B_{n}$ for this MPL crossing is not significantly different from zero. Generally, the mean of $B_{n}$ should exceed 2-3 0 to be significant. The B1-B2 hodogram shows a view of the tip of the magnetic field vector projected on to a plane tangent to the MPL. This projection shows how the field rotates in the plane tangent to the MPL through the inner half of the MPL. The field magnitude in the MPL drops no less than $10 \gamma$ while electron density values, sampled every $\sim 3.1 \mathrm{~s}$, remain near their magnetosheath values through the MPL. The magnetopause normal direction is $\phi_{\mathrm{SE}}=322^{\circ}$ and $\lambda_{\mathrm{SE}}=-21^{\circ}$, consistent with the dawn-side location of this crossing.

Figures $2 \cdot 3 \cdot 2.5$ and $2 \cdot 3 \cdot 2.6$ provide azimuthal averages of the velocity distribution from the magnetosheath (MS) to the magnetosphere (MG) for the 20 June 1972 crossing. These azimuthal averages are taken about the centroid of the distribution (i.e., these averages are made in the 

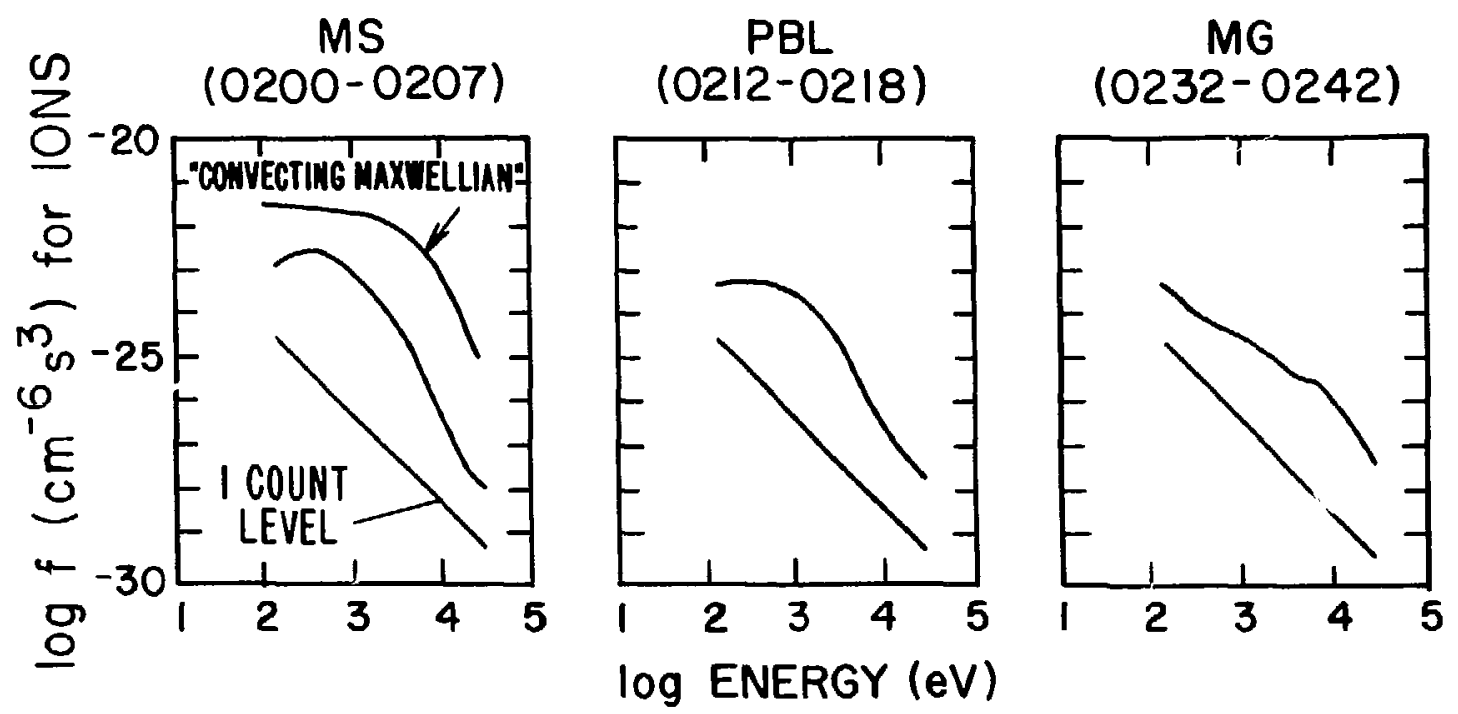

Figure 2.3.2.5 Azimuthal averages of the ion distribution function plotted for six to ten minute intervals for the 20 June 1972 crossing of the MPL-PBL region. 
IONS
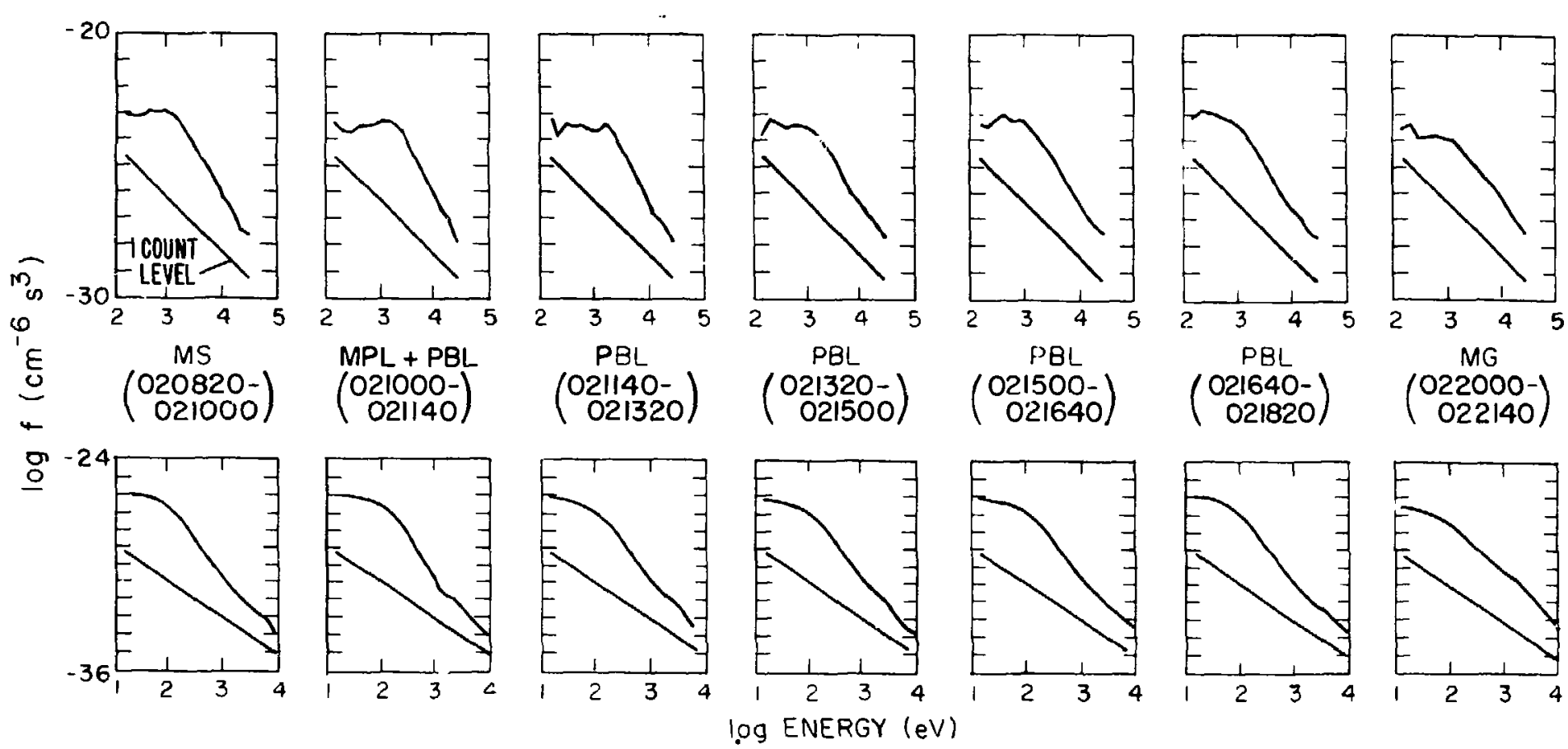

ELECTRONS

Figure 2.3.2.6 Azimuthal averages of the distribution function plotted for $\sim 100 \mathrm{~s}$ intervals for the 20 June 1972 crossing. 
frame of the moving plasma) and are plotted as phase space density $f$ versus energy. For comparison, a "convecting Maxwellian" distribution function (Maxwellian distribution calculated in the roving frame of the plasma) is marked on the first plot of the azimuthal average of $f$. Measured velocity distributions in the magnetosheath and PBL are distinctly non-Maxwellian. Above a given energy ( $100 \mathrm{eV}$ for electrons and $\sim 500 \mathrm{eV}$ for ions), the energy spectra often closely approximate a power law shape. Since this is a collisionless plasma environment, nonMaxwellian distributions can be maintained over large spatial scales depending on the plasma source and propagation characteristics. In the long time averages shown in Figure 2.3.2.5, the PBL spectra are much more similar to the magnetosheath spectra than they are to the magnetospheric spectra.

A sequence of ion and electron spectra is shown in Figure 2.3.2.6. These spectra show azimuthal averages of the two-dimensional ion and electron velocity distributions. The PBL and magnetosheath spectra are closely related in these $100 \mathrm{~s}$ averages. This spectral similarity is even more evident in the electron differential spectra, Figure 2.3.2.7, which are plotted every $\sim 3.1 \mathrm{~s}$ in groups that cover one spin period each. The striking continuity in spectra observed across the MPL is almost always observed in the IMP 6 data. By comparison, the nearby magnetospheric spectra show a higher mean energy. The velocity distributions and electron spectra for the 6 June 1972 crossing indicate a very close relationship of magnetosheath and PBL plasma. 

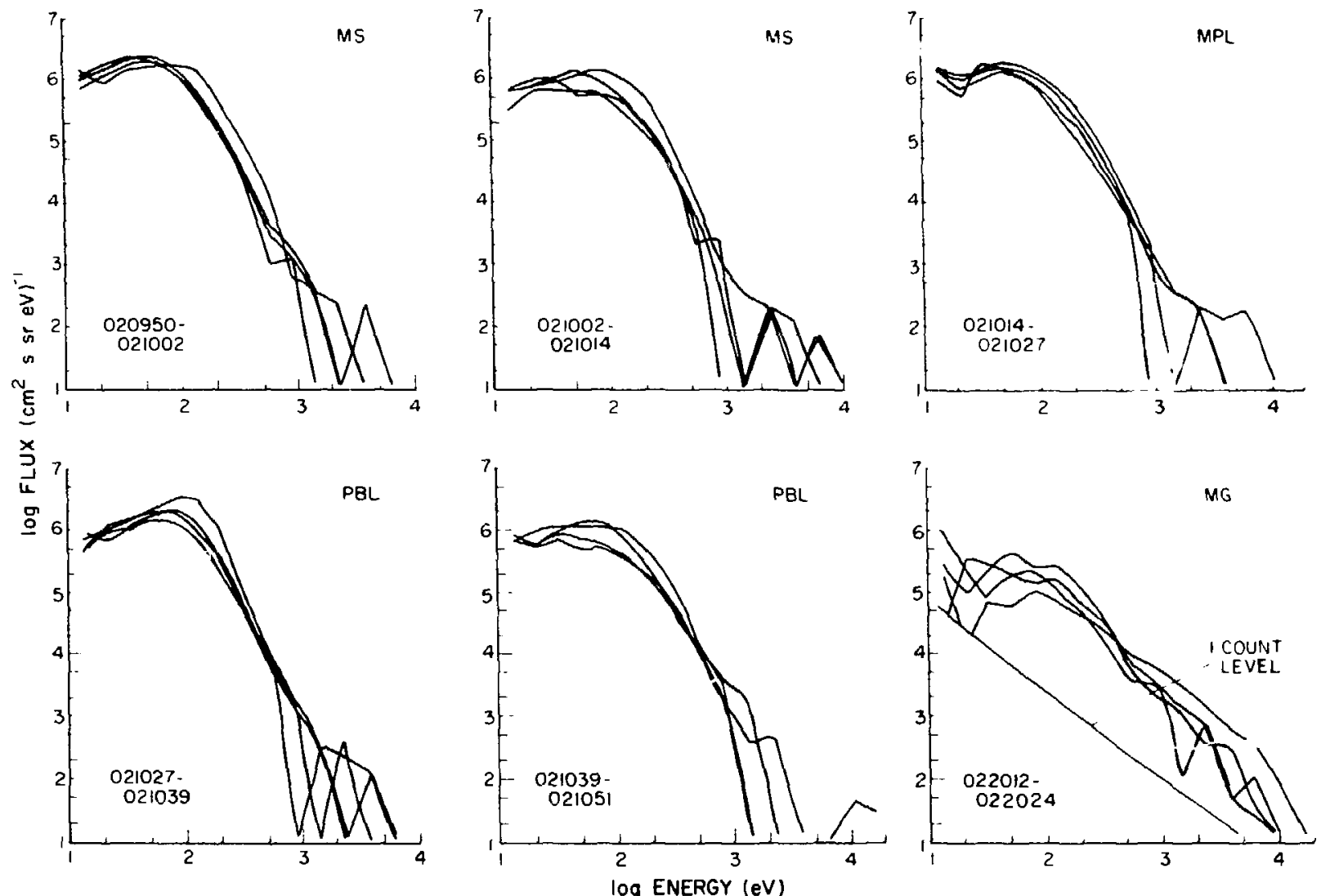

Figure 2.3.2.7 Electron differential spectra plotted four times every $\sim 12.5 \mathrm{~s}$ at successive locations near the MPL for the 20 June 1972 crossing. Nearby magnetospheric spectra are also plotted for comparison. 
Electron density and energy spectra at $s 3.1-s$ time resolution and velocity distributions show no distinctive change across the MPL. Whereas the proton cycloiron racius is $s 145 \mathrm{~km}$ in this PBL, the electron cyclotron radius is only $\sim 3 \mathrm{~km}$. Effective neutrality of the plasma is steadily enforced by the fast-moving (s $1 R_{E} / s$ ) electrons for any plasma in which the characteristic scale lengths are much greater than the Debye length, $\lambda_{D}(\approx .01 \mathrm{~km})$, which is much less than the ion cyclotron radius or any characteristic scale length. Thus, energy spectra of this quasi-neutral plasma closely reflect source populations. These observations indicate that a major plasma source for the PBL is the magnetosheath and that the PBL close to the MPL is composed primarily of plasma that has recently traversed the current layer.

A depletion in plasma density occurs in the magnetosheath close to the MPL in this crossing. Similar observations of a plasma depletion layer are fairly common and have been recently described by Crooker et al. (1978). Plasma depletion can be caused by magnetic flux tube compression as the field becomes draped against the magnetopause. Observed anisotropy values with $\mathrm{P}_{\perp}>\mathrm{P}_{\|}$are predicted by the flux tube compression model of Zwan and Wolf (1976). For the 20 June 1972 crossing, enhanced ion anisotropy is present in the nearby magnetosheath and a distinct enhancement in electron anisotropy occurs at the MPL. 


\subsubsection{The 1 May 1973 IMP 6 Crossing}

This low-latitude, dusk-side crossing by IMP 6 shows a marked density decrease (Figure 2.3.3.1) at the MPL. As noted for the previous two crossings, the scale length for changes in the plasma parameters is roughly equal to the PBL thickness, whereas the current layer thickness is significantly smaller.

The MPL is clearly visible in the magnetic field data (Figures 2.3.3.2 and 2.3.3.3). The cross-field flow component in the PBL is substantial in this crossing with flow directions that are 90 to $150^{\circ}$ away frow the field direction (even accounting for the possible flow directions that could be sampled by the $\pm 45^{\circ}$ electrostatic analyzer fan). Pitch-angle distributions presented in Figure 2.3.3.4 show a clear pancake-shaped or loss cone distribution in the PBL, the nearby magnetosphere and the magnetosheath as well. This example demonstrates that a loss cone distribution is not a sufficient condition for the presence of closed field lines (see section 2.4 .6 ). Considering the field direction and the energetic electron characteristics, however, these observations are most compatible w:th a PBL that is located primarily on closed field lines.

Magnetic field hodograms for this crossing are presented in Figure 2.3.3.5. The normal fiest component is near zero although the field magnitude remains high acl:ss the MPL. The magnetopause normal direction is $\phi_{\mathrm{SE}} \simeq 3^{\circ}$ and $\lambda_{\mathrm{SE}} \approx-63^{\circ}$, consistent with the crossing location. 


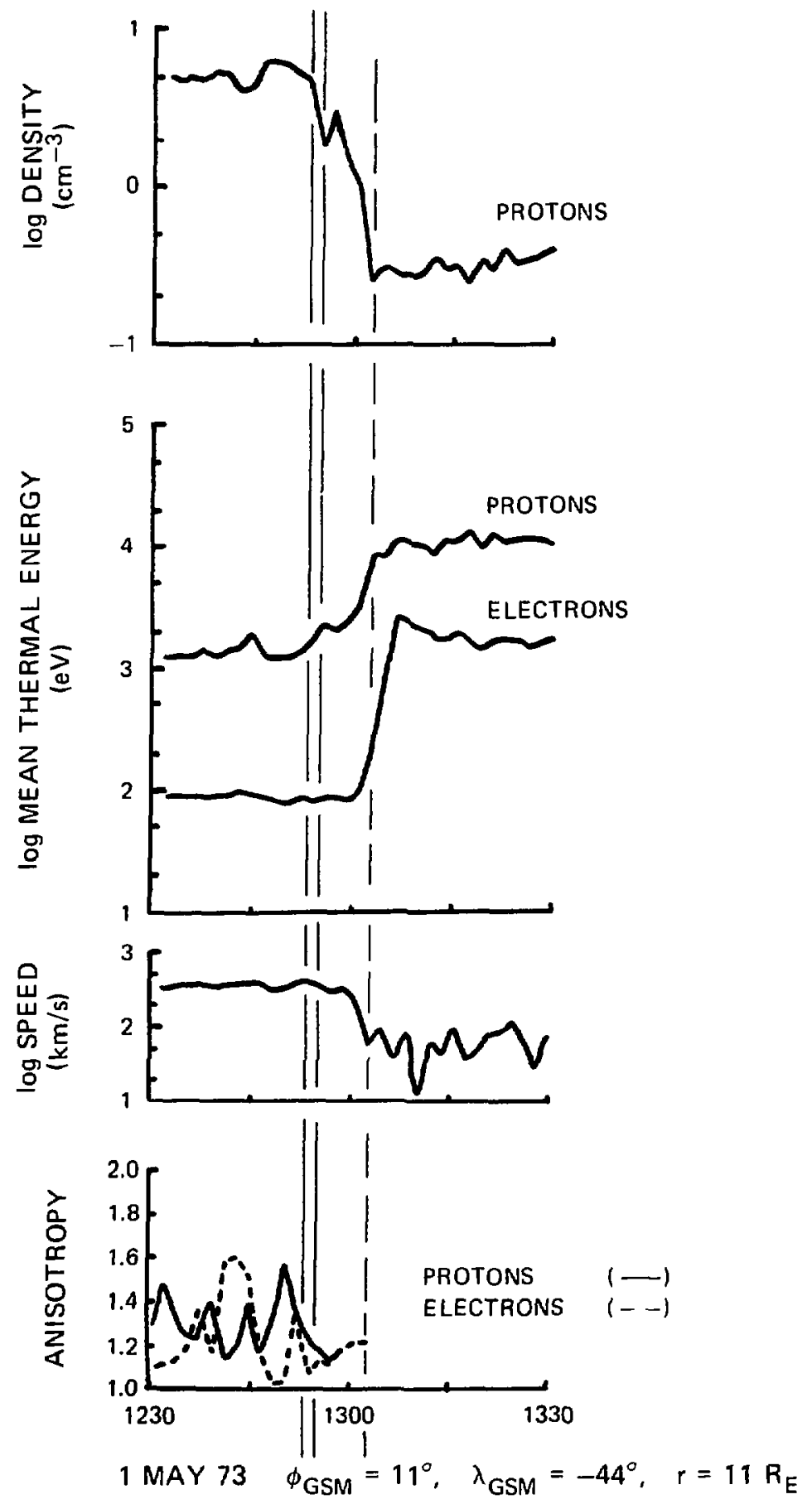

Figure 2.3.3.1 1 May 1973 plasma data. 


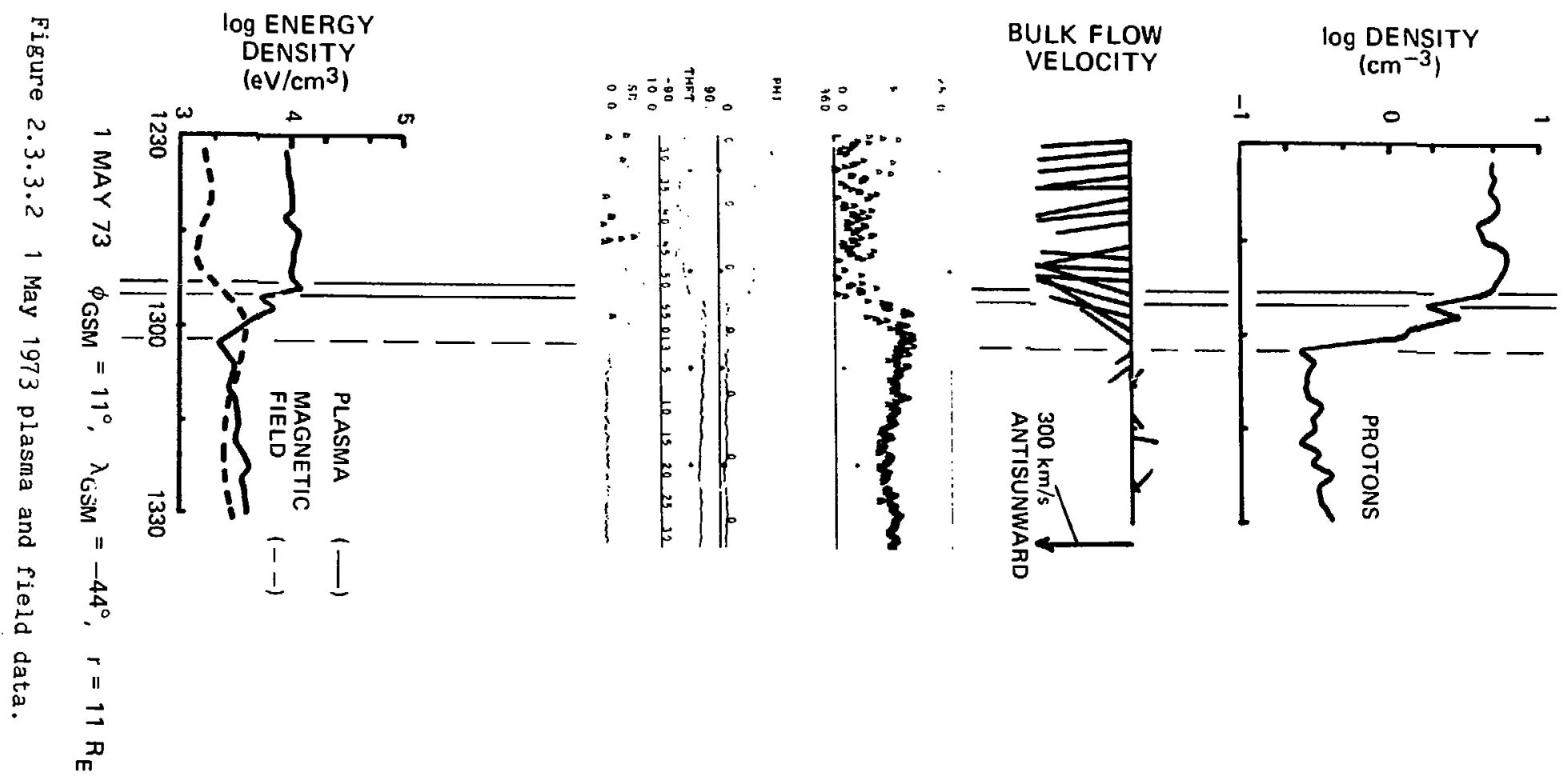



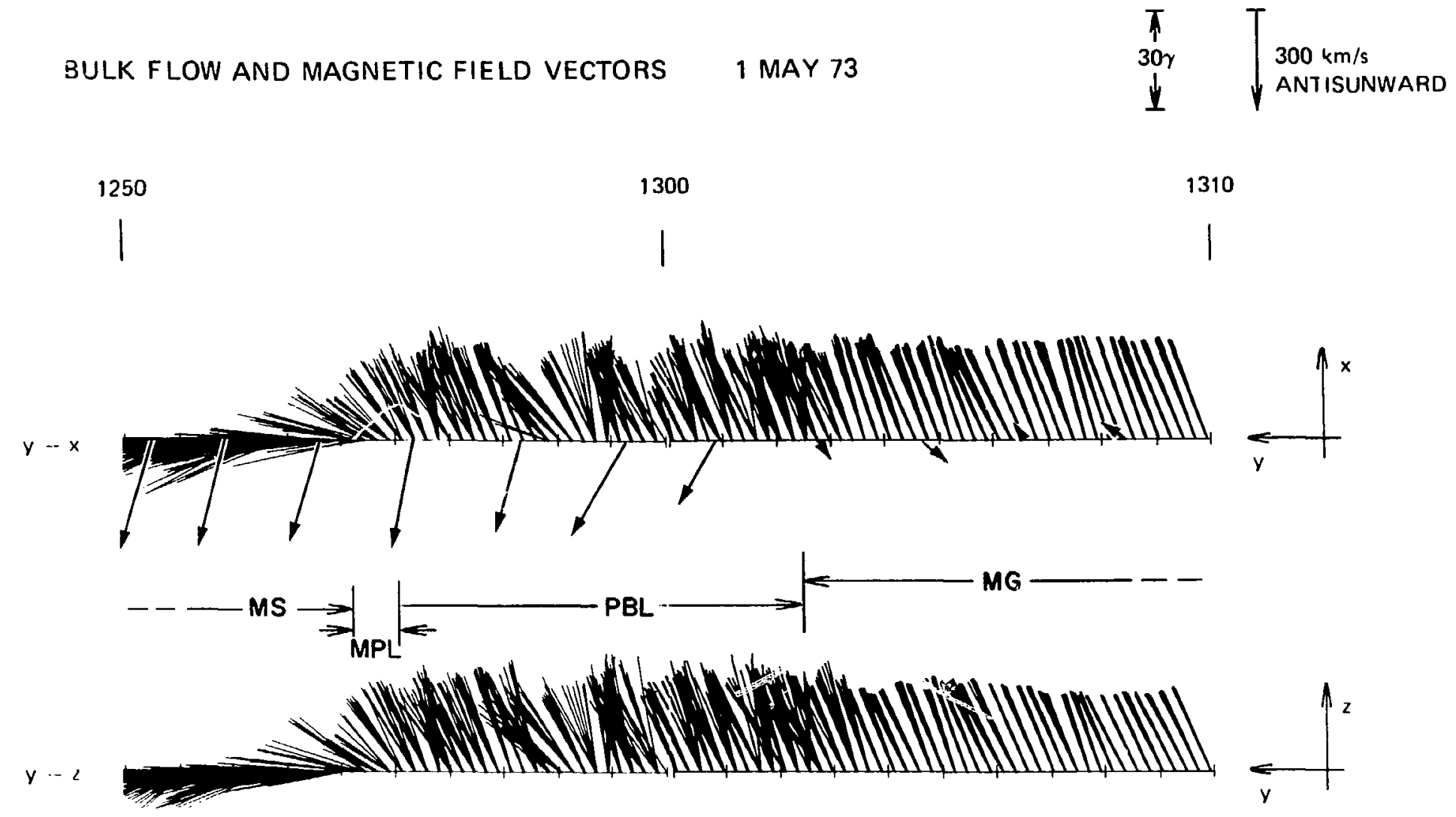


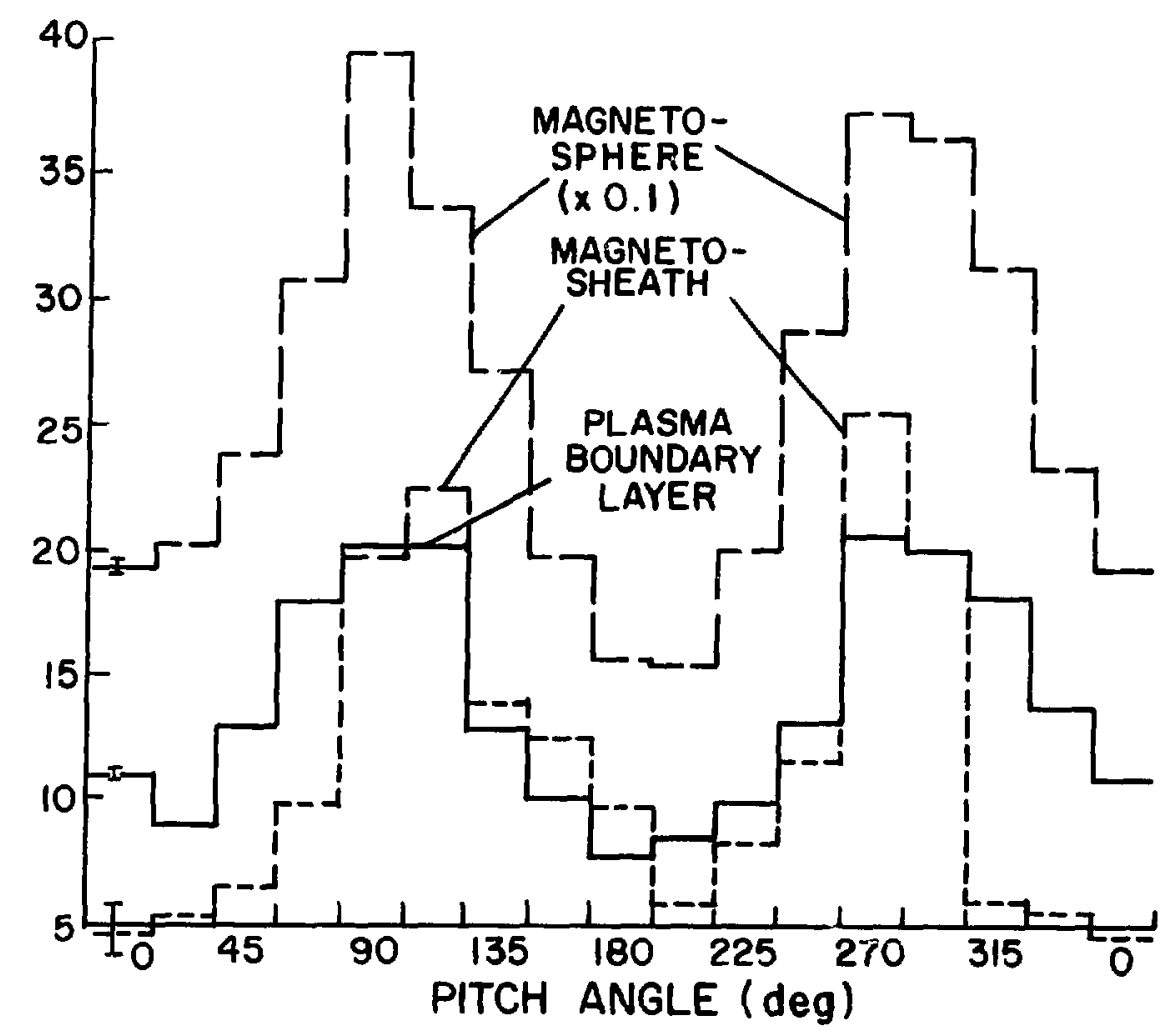

Figure 2.3.3.4 Energetic electron (47- to 350-keV) pitch-angle distributions for the 1 May 1973 crossing. 

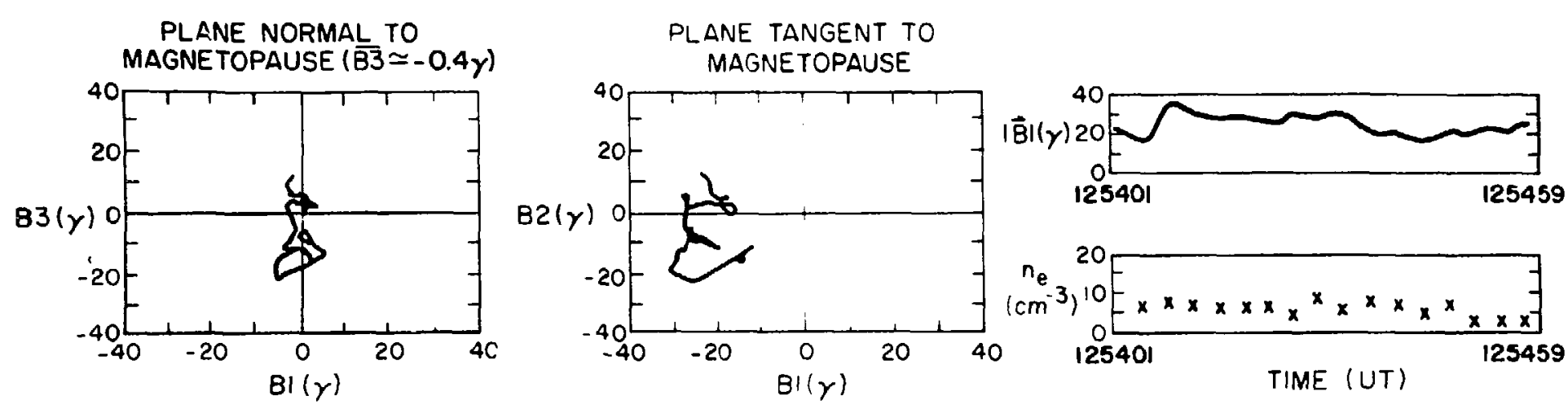

Figure 2.3.3.5 Magnetic field hodograms, field magnitude and electron density for the 1 May 1973 crossing of the MPL. 
Many other crossings also illustrate how the MPL is, in general, distinguished by a field direction change and not by changes in field magnitude. The continuity in electron density through the MPL shown in Figure $2.3 \cdot 3 \cdot 5$ is also a common feature.

Electron differential energy spectra sampled across the MPL are shown in Figure 2.3.3.6 and they show a clear continuity across the MPL. This signature would be very improbable if a relatively distant source supplied the PBL immediately inside the current layer. Magnetospheric spectra are distinctly different and show a higher mean energy. Ion and electron energy spectra, plotted as azimuthal averages of the velocity distribution, are shown in Figure 2.3.3.7. These spectra trace plasma characteristics from the magnetosheath, through the MPL and PBL, to the magnetosphere. In general, the MPL and PBL spectra are similar to the nearby magnetosheath spectra, although the PBL spectrum sampled adjacent to the magnetosphere is similar to the magnetospheric spectrum. This effect may be caused by mixing with magnetospheric plasma. 

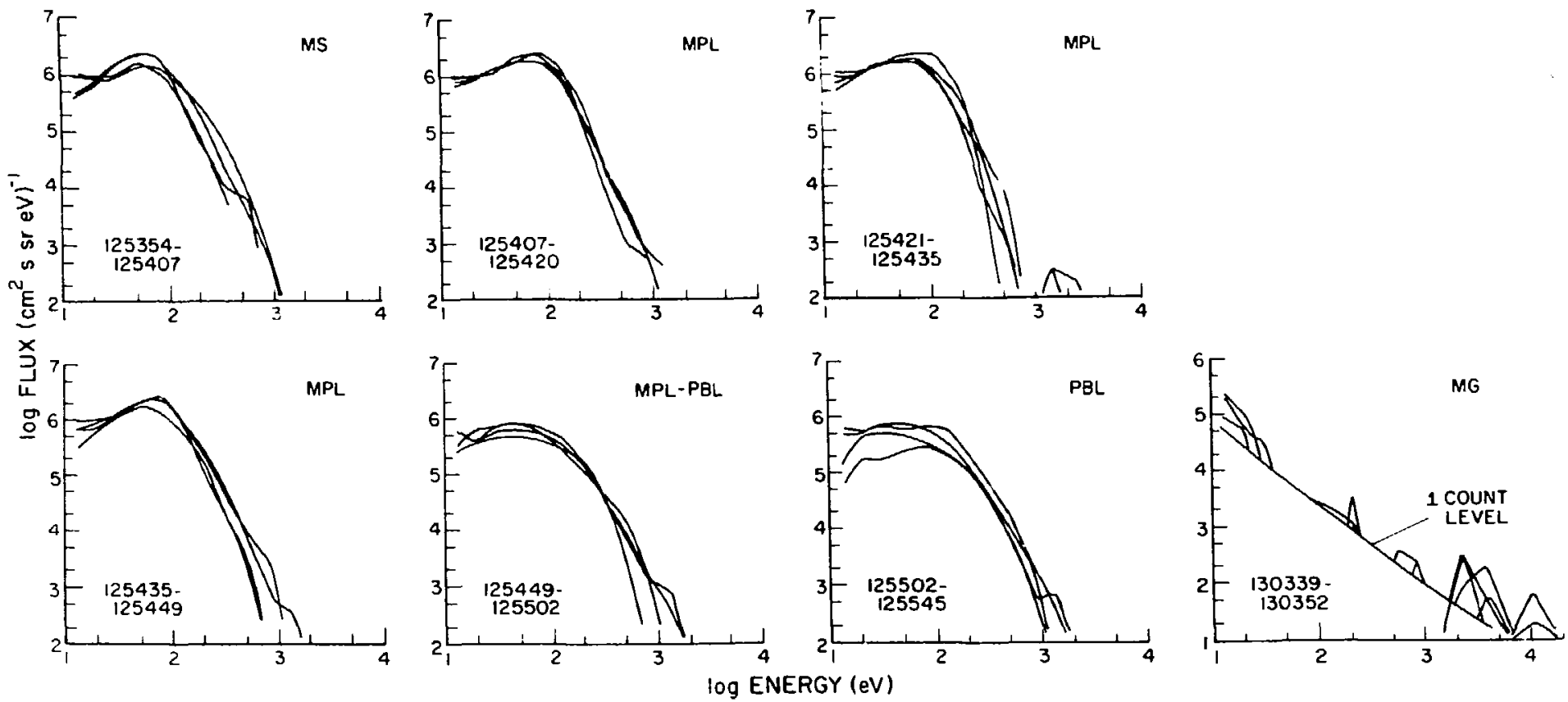

Figure 2.3.3.6 Electron differential spectra plotted four times every $\sim 12.5 \mathrm{~s}$ at successive locations near the MPL for the 1 May 1973 crossing. Nearby magnetospheric spectra are also plotted for comparison. 
IONS
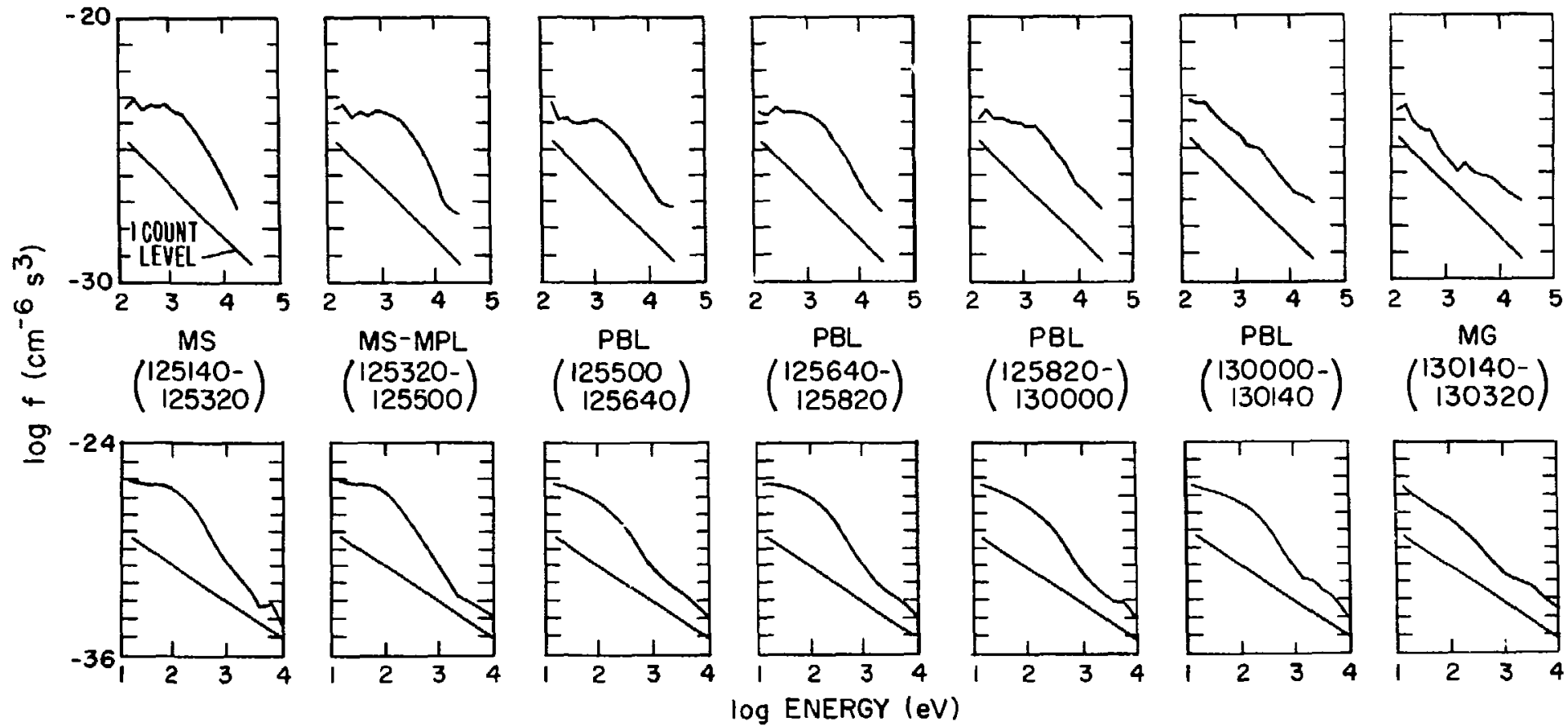

ELECTRONS

Figure 2.3.3.7 Azimuthal averages of the distribution function plotted for $\sim 100 \mathrm{~s}$ intervals for the 1 May 1973 crossing. 


\subsubsection{The 16 June 73 IMP 6 Crossing}

This low latitude dawn side crossing (Figures 2.3.4.1-.3) does not have a clear MPL. A series of field direction changes before 0333 UT could mark a multiple magnetopause crossing. Based on plasma density, however, an outer boundary near 0402 UT should be selected. Tnis latter identification is consistent with energetic electrons that show pancake-shaped pitch angle distributions after 0402 UT and an antisunward streaming of energetic electrons before that time (Figure 2.3.4.4). Energetic electron intensities in the PBL progressively increase while the satellite continues inward. These pitch-angle distributions suggest that the PBL (after 0403 UT) is on closed field lines (see section 2.4.6). One could, however, choose the outer PBL boundary at $\backsim 0330$ UT and state that the inner PBL is on closed field lines while the outer PBL is on open field lines.

Plasma flow directions in the closed field line region of the PBL (in either interpretation discussed above) show a substantial crosefield component as shown in Figure 2.3.4.3. Typical cross-iield flow angles are $\sim 90$ to $130^{\circ}$, depending on the exact flow direction considered that could be sampled by the $\pm 45^{\circ}$ aperture of the electrostatic analyzer.

Azimuthally averaged ion and clectron energy spectra sampled across the PBL (Figure 2.3.4.5), show the similarity of spectral shapes from the magnetosheath through the PBL and the contrast with the nearby magnetospheric spectra. The lack of a distinct inner or outer boundary to the PBL suggests the presence of a diffusion mechanism that can contribute to the plasma population of the PBL (section 3.3). 


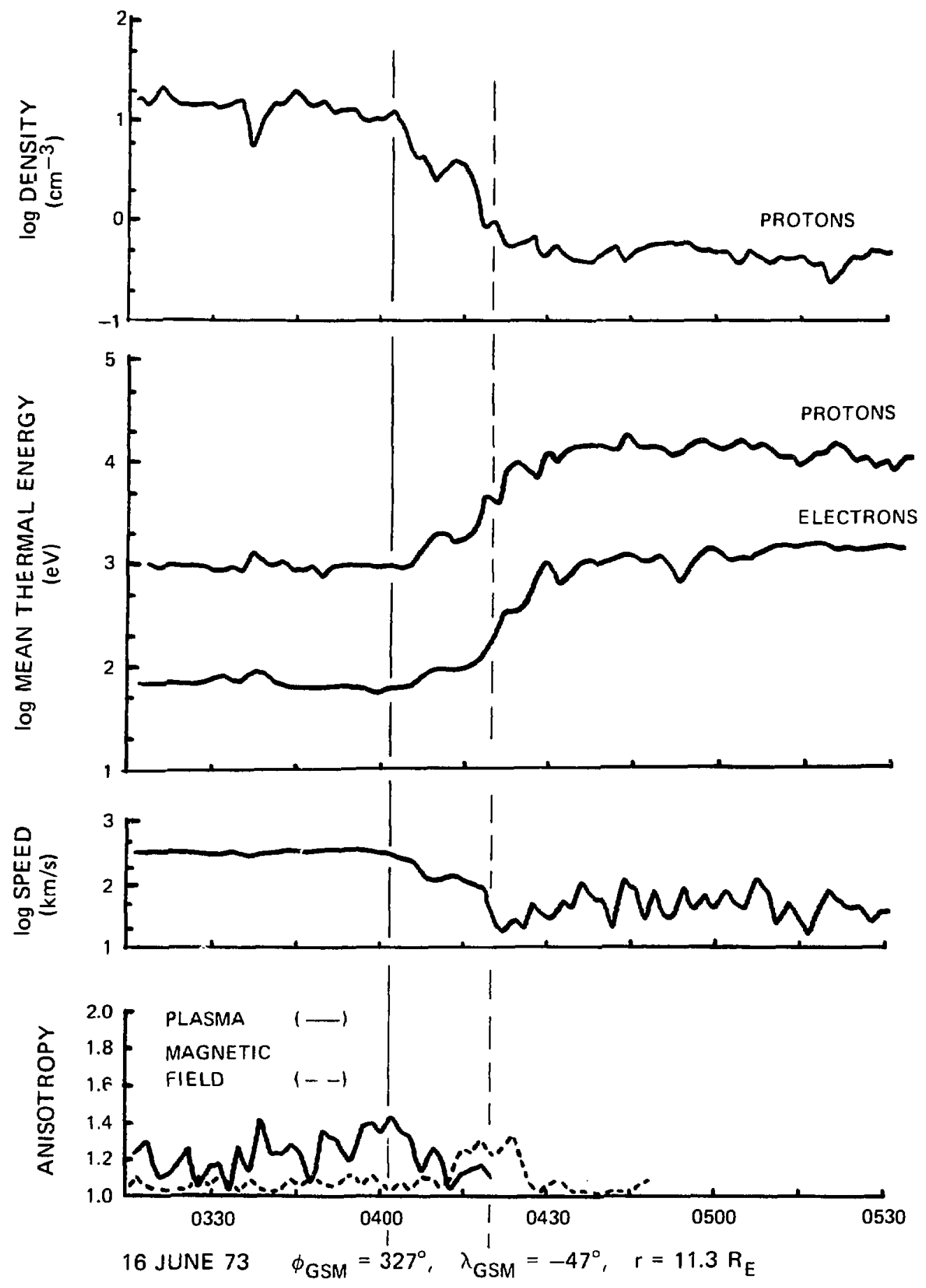

Figure 2.3.4.1 16 June 1973 plasma data. 


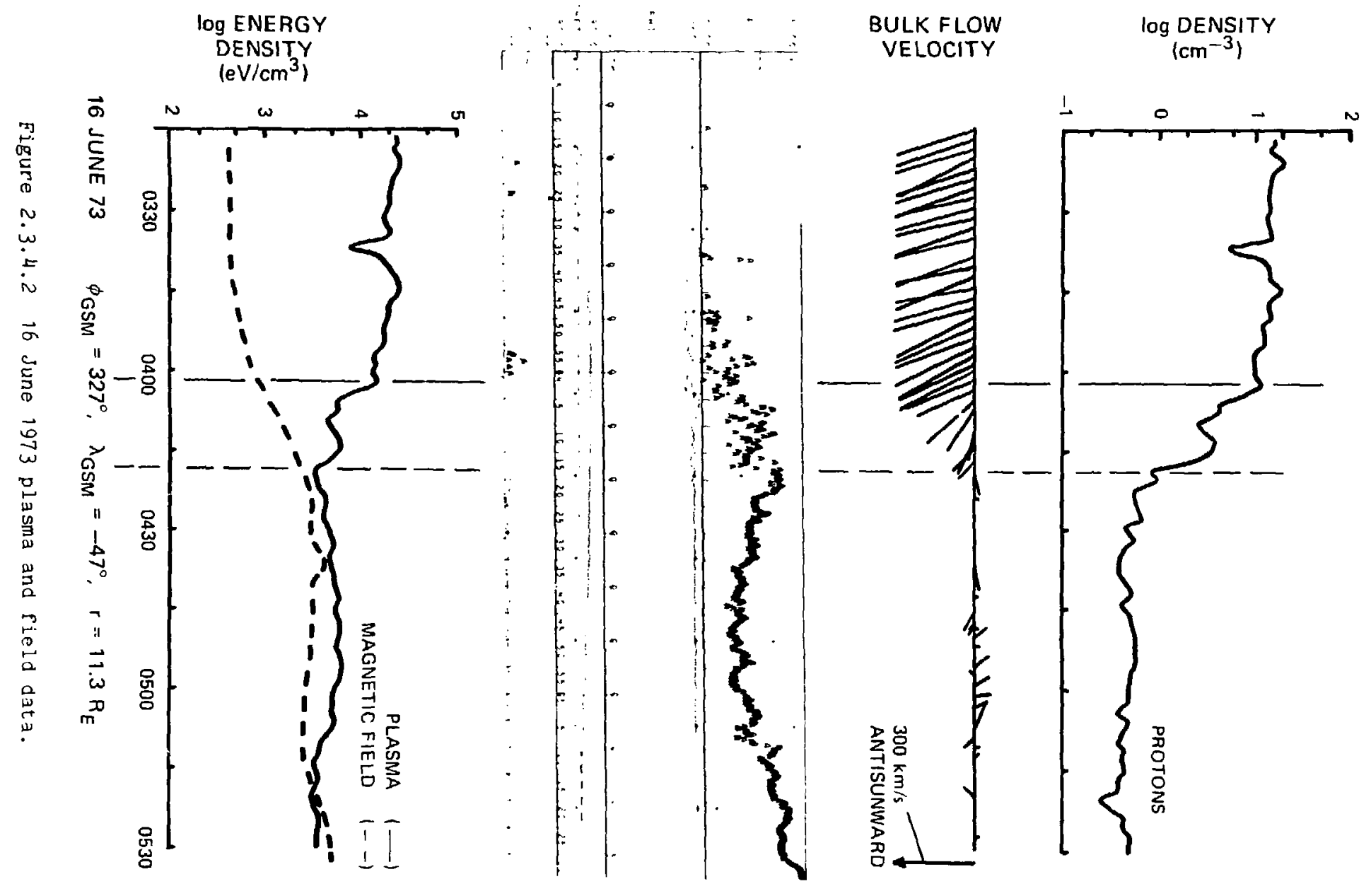


BULK FLOW AND MAGNETIC FIELD VECTORS 16 JUNE 73
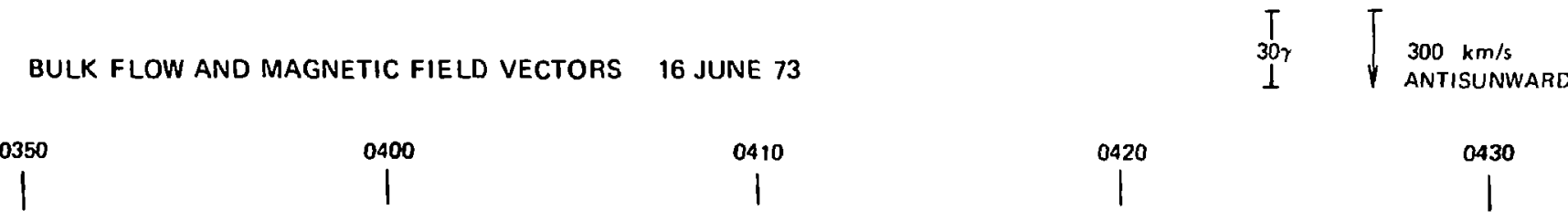

1

0420

0430

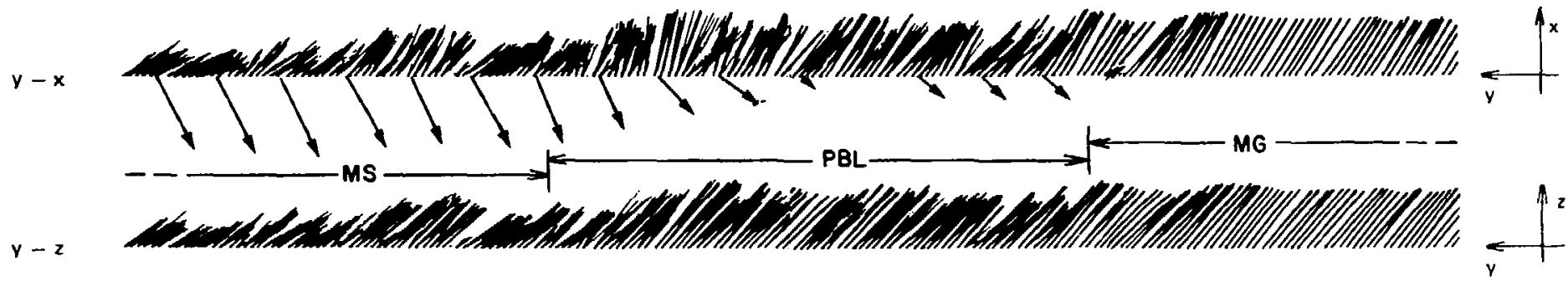

Figure 2.3.4.3 16 June 1973 plasma flow and field projections. 

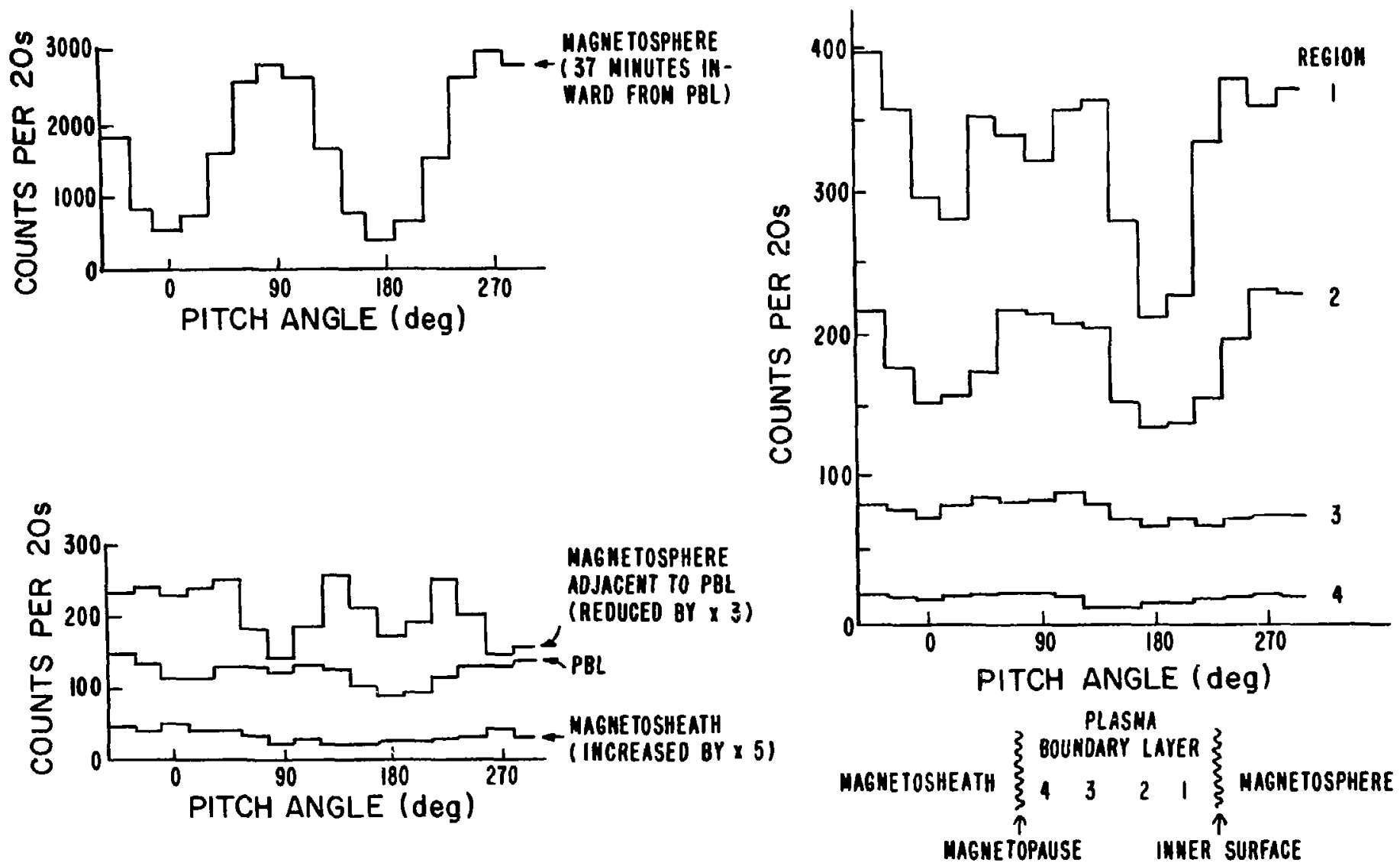

Figure 2.3.4.4 Energetic electron (47- to 350-keV) pitch-angle distributions for the 16 June 1973 crossing. 
IONS
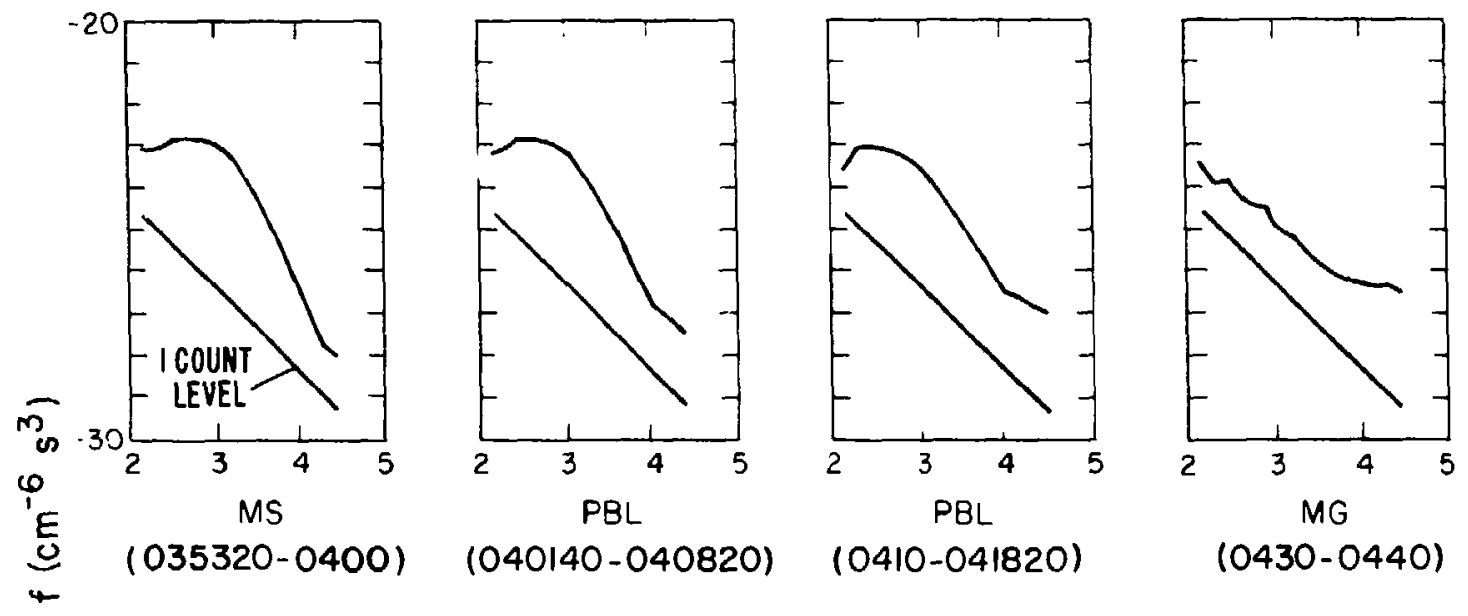

(0430-0440)
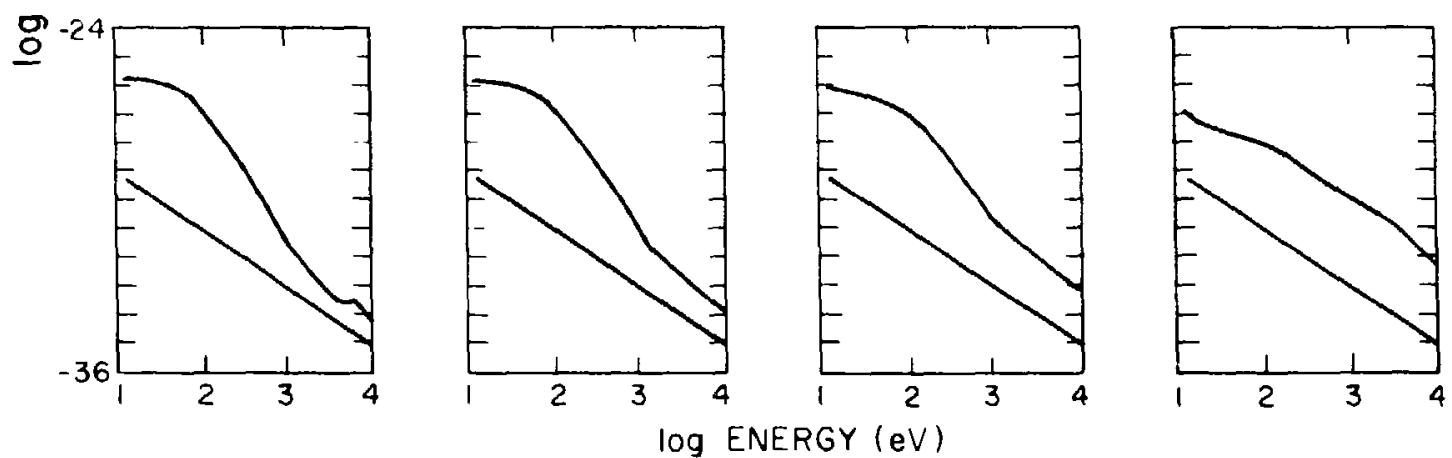

ELECTRONS

Figure 2.3.4.5 Azinuthal averages of the distribution function plotted for $\sim 100 \mathrm{~s}$ intervals for the 16 June 1973 crossing. 


\subsubsection{April 1972 IMP 6 Crossing}

This dusk side crossing (Figures $2 \cdot 3 \cdot 5.1$ and 2.3 .5 .2 ) is in the subsolar region within about $20^{\circ}$ or $\sim 5 \mathrm{R}_{E}$ of the subsolar point. A clear MPL signature is present in the magnetic field data as a direction change of $\vec{B}$ near 1617 UT. There is no distinct change in the plasma parameters at the MPL except for increased deflection of bulk flow direction relative to the $\mathrm{X}_{\mathrm{GSM}}$ axis.

Figure 2.3.5.3 shows ecliptic plane projections of the magnetic field and bulk plasma flow. A substantial normal component of plasma flow is indicated, irrespective of the flow component out of the ecliptic plane.

A significant normal field component $(\overline{B 3} \simeq 8 \gamma$ ) is indicated by the minimum variance analysis as shown by the hodograms in Figure 2.3.5.4. Both electron density and field magnitude remain high through the MPL as shown on the right side of Figure 2.3.5.4. Field magnitude changes over a length scale comparable to that of the PBL, whereas the field direction change occurs over a relatively small distance. Similarly, the plasma and field energy density change over a length scale comparable to that of the PBL.

The mean thermal energy and plasma density retain their magnetosheath levels until the satellite approaches the inner PBL surface. This suggests that the $P B L$ in this case is made up primarily of magnetosheath plasma. Ion and electron energy spectra, shown in Figure 2.3.5.5, illustrate the close similarity of PBL and magnetosheath spectra and the contrast with spectra for the nearby magnetospheric plasma. The progression of $3.1-\mathrm{s}$ time resolution electron differential spectra 


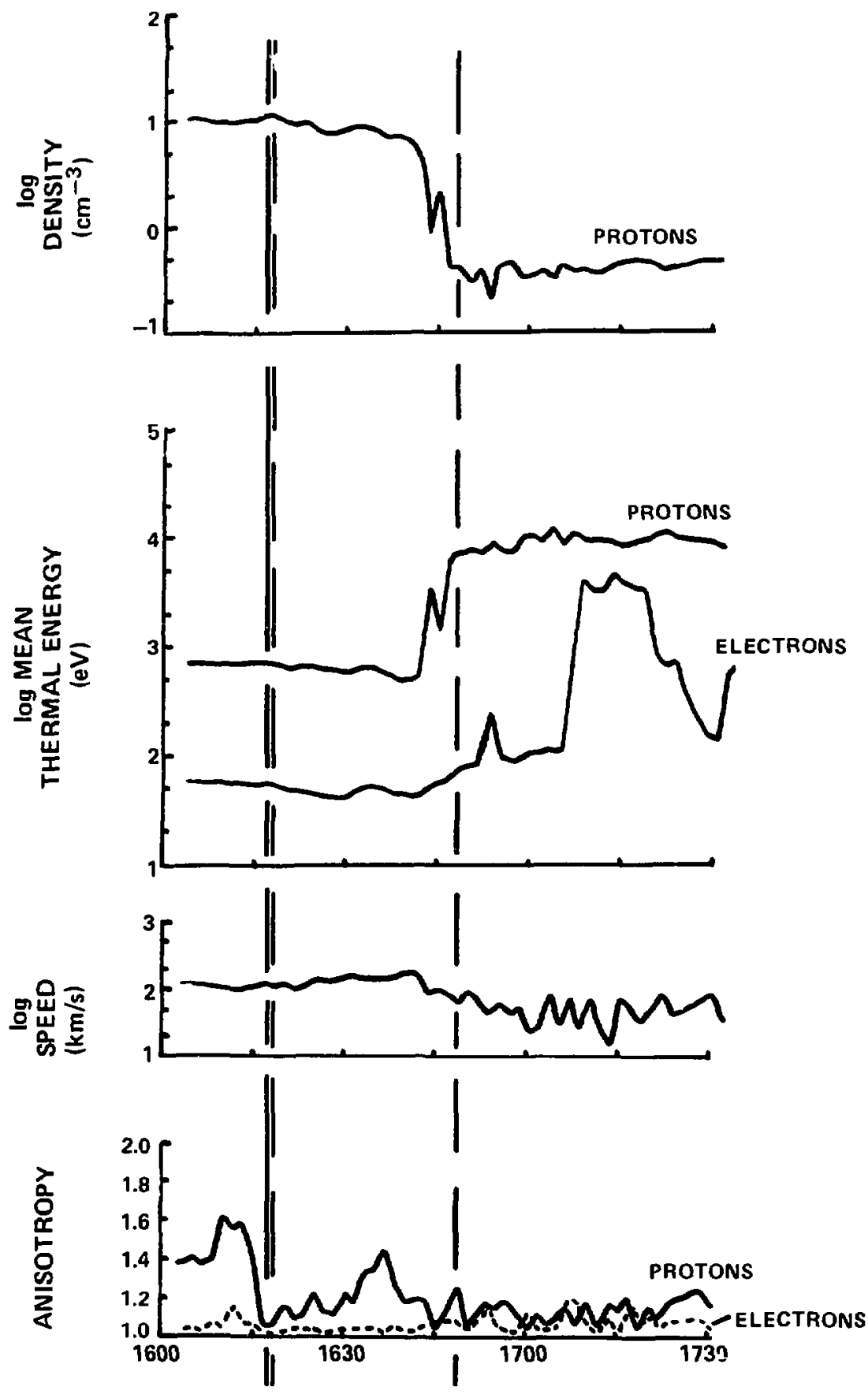

14 APRIL $72 \phi_{G S M}=19^{\circ}, \lambda_{G S M}=-23^{\circ}, r=12.7 R_{E}$

Figure 2.3.5.1 14 April 1972 plasma data. 


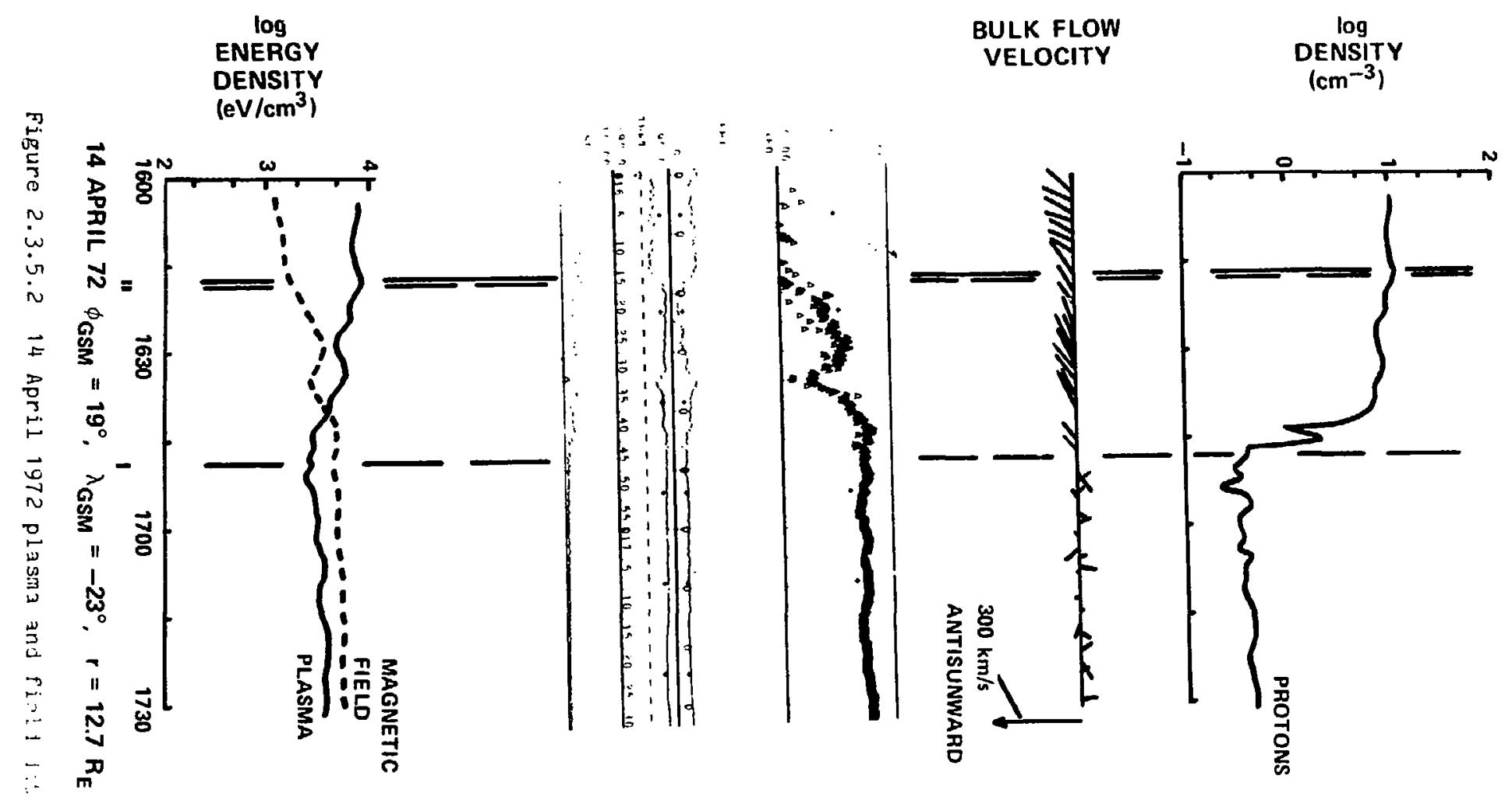


BULK FLOW AND MAGNETIC FIELD VECTORS

14 APR 72

$\underset{1}{30 \%}\left\lceil\begin{array}{l}300 \mathrm{~km} / \mathrm{s} \\ \text { ANTISUNWARD }\end{array}\right.$

$\begin{array}{ccccc}1610 & 1620 & 1630 & 1640 & 1650\end{array}$

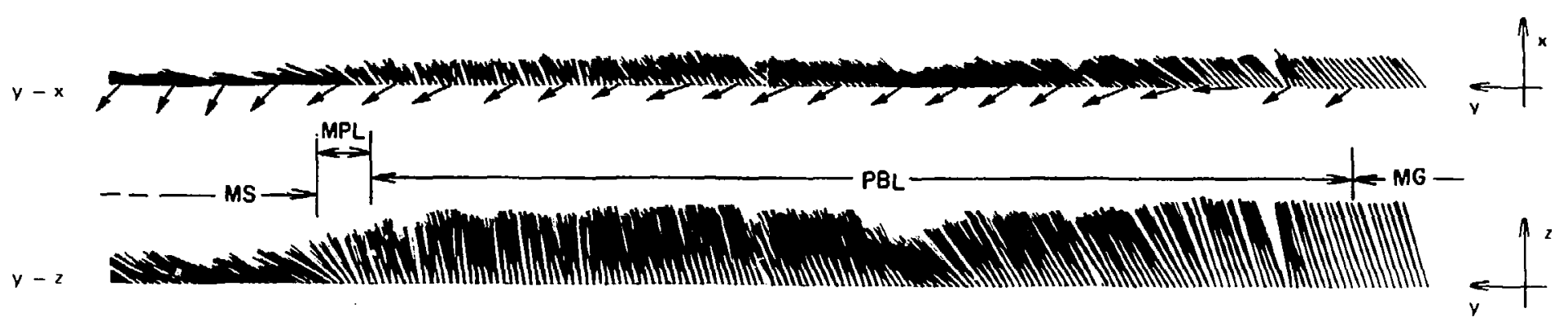

Figure 2.3.5.3 14 April 1972 plasma flow and field projections. 

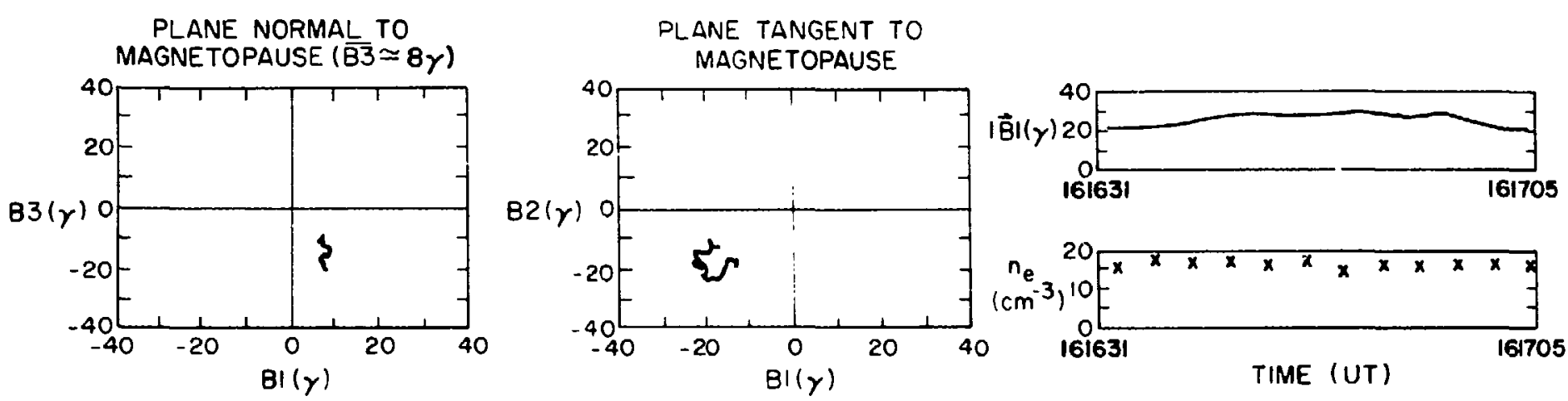

Figure 2.3.5.4 Magnetic field hodograms, field magnitude and electron density for the 14 Apr:1 1972 crossing of the MPL. 

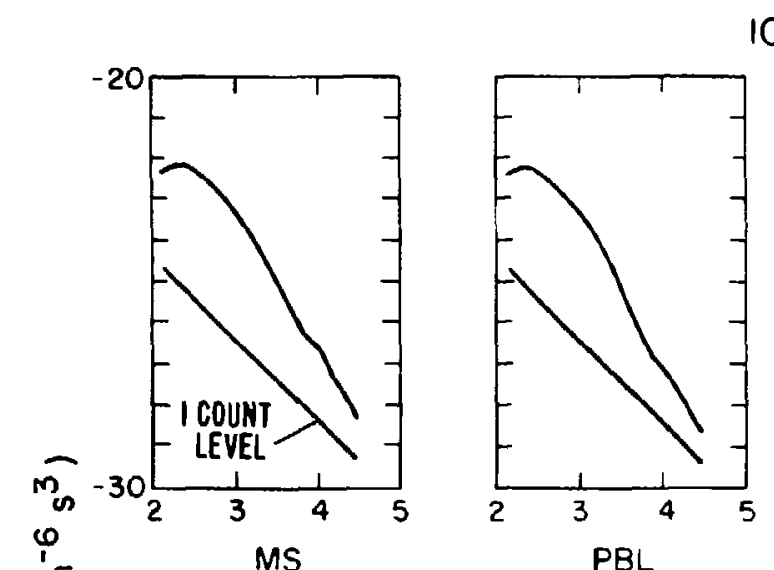

IONS

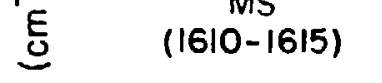

(162320-1630)
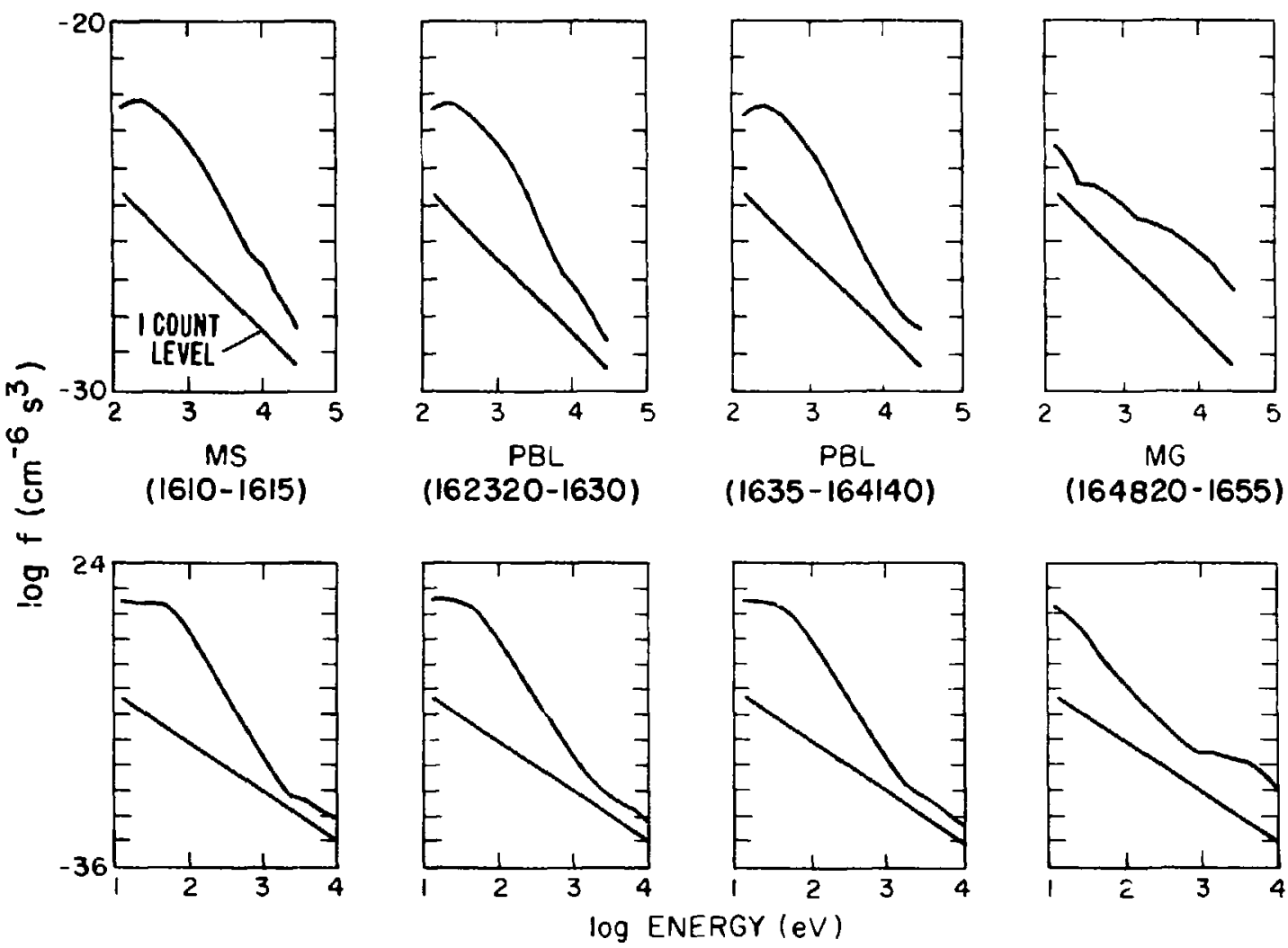

(164820-1655)

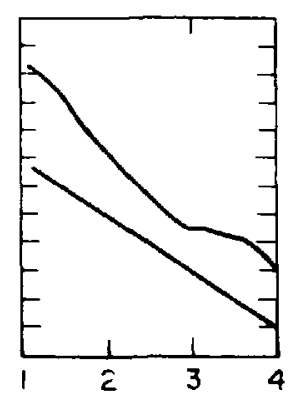

ELECTRONS

Figure 2.3.5.5 Azimuthal averages of the distribution function plotted for five to seven minute intervals for the 14 April 1972 crossing. 
across the MPL is illustrated in Figure 2.3.5.6 for four spin periods. These spectra again indicate the close relationship of PBL and magnetosheath plasma in the presence of a clear current layer signature. Magnetospheric spectra are very different from spectra sampled near the MPL.

The antisunward-somponent plasma flow observed in the PBL in this crossing is not compatible with a cusp region source because of (1) the crossing location, which requires a local or subsolar region source for the magnetosheath-like plasma observed in the PBL, and (2) the continuity in plasma parameters, including energy spectra, across the MPL. Locally observed magnetospheric plasma cannot be a significant contributor to the PBL plasma population because the magnetospheric plasma density is reduced by more than a factor of 50 relative to the outer PBL, and because the mean thermal energy is nearly at the magnetosheath level except for the innermost $20 \%$ of the PBL. 


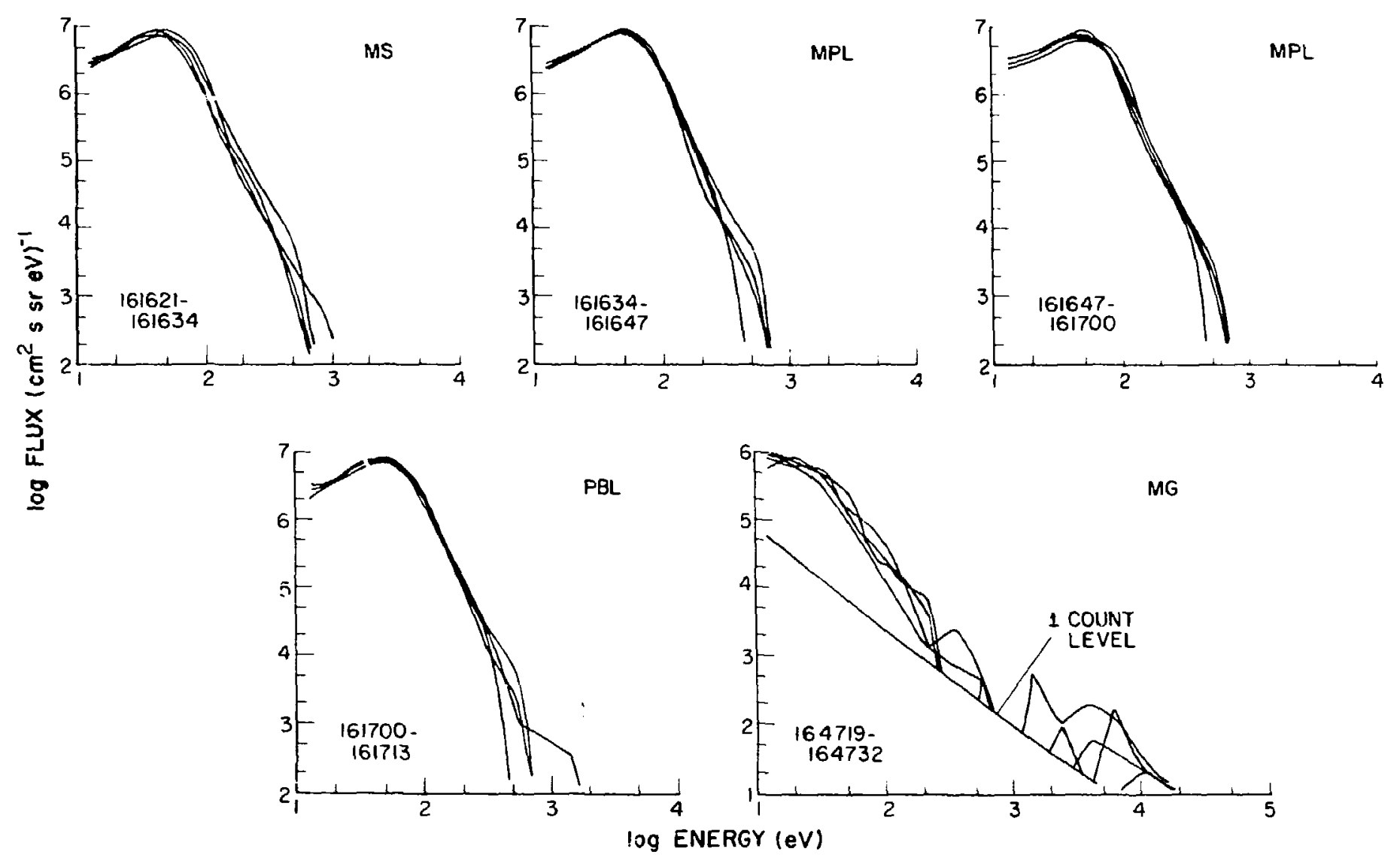

Figure 2.3.5.6 Electron differential spectra plotted four times every $\sim 12.5 \mathrm{~s}$ at successive locations near the MPL for the 14 April 1972 crossing. Nearby magnetospheric spectra are 


\subsubsection{May 1972 IMP 6 Crossing}

This is a noon-meridian, Low latitude MPL-PBL crossing (Figures 2.3.6.1-3) that is in an exceptional location for observing plasma flow relative to the cusp region. Based on the field direction $\left(\phi_{B} \sim 20^{\circ}, \lambda_{B}\right.$ $\sim 40^{\circ}$ ) the satellite position is near the noon meridian immediately equatorward of the cusp. The bulk plasma flow is nearly field-aligned as shown in Figure 2.3.6.3, and is directed toward the cusp. A clear MPL is located near 0202 UT with two other possible excursions into the MPL within five minutes before this time.

There is a significant density decrease near the MPL, as shown in Figure 2.3.6.4, with $\sim$ 3.1-s time resolution electron density samples. This density decrease is accompanied by an unusually abrupt increase in magnetic field magnitude near the MPL (Figure 2.3.6.2), in contrast with the common observation thät, the length scale of the $\mid$ B $\mid$ gradient is often closer to the PBL thickness than to the MPL thickness. The magnetic field hodogram exhibits a near-zero normal field component considering the variations in B3. The direction of minimum $B$ variasce, B3, was $\phi_{\mathrm{SE}} \curvearrowright 174^{\circ}$ and $\lambda_{\mathrm{SE}}=44^{\circ}$.

Figure 2.3.6.5 shows the range of observed ion and electron energy spectra from the magnetosheath through the PBL and also compares the spectra of the higher mean thermal energy magnetospheric plasma. PBL spectra in this crossing exhibit a local decrease near $500 \mathrm{eV}$. This decrease indicates that PBL plasma not immediately adjacent to the MPL could have been supplied from more distant points in the subsolar region.

PBL plasma immediately adjacent to the MPL has spectra essentially 


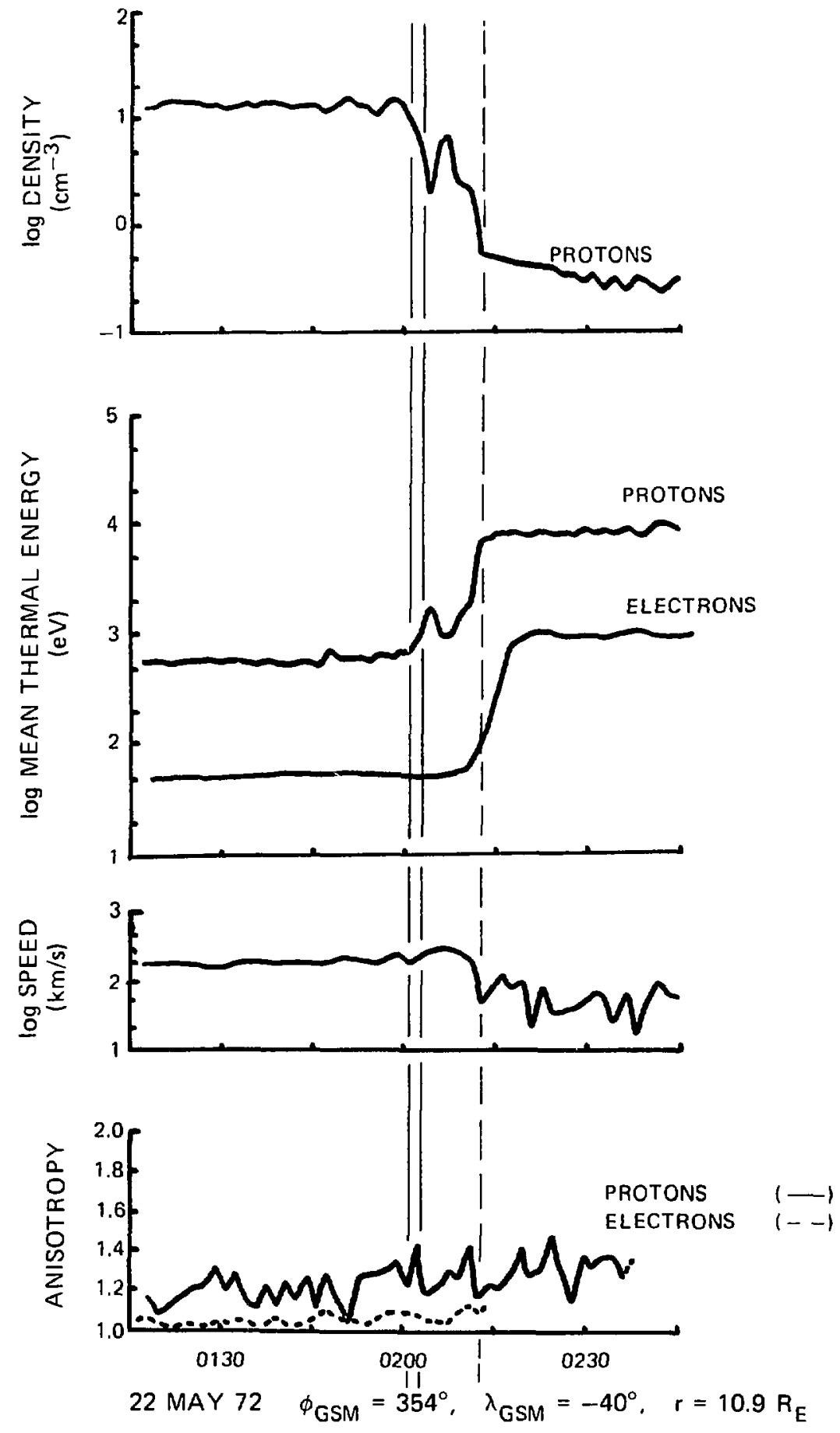

Figure 2.3.6.1 22 May 1972 plasma data. 

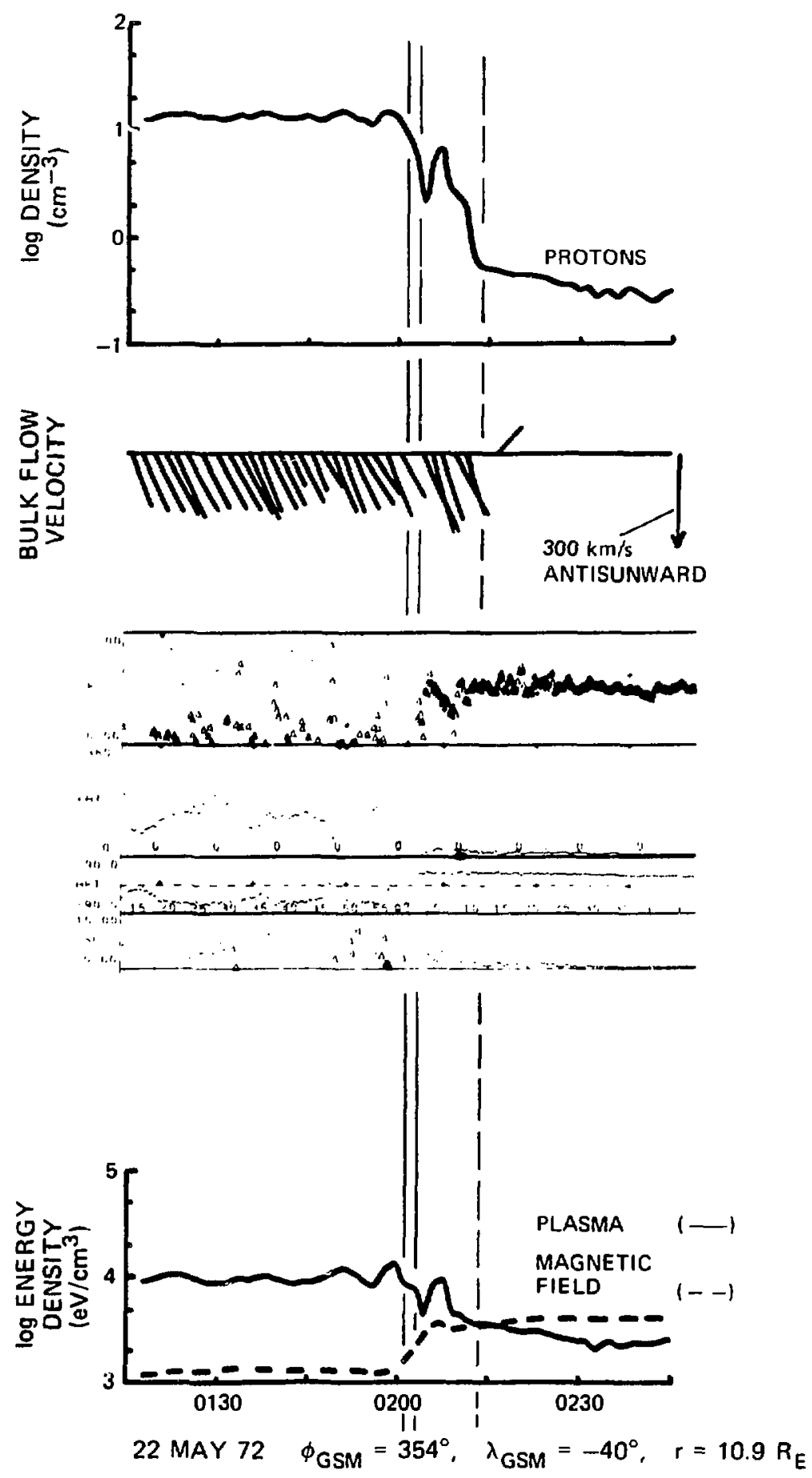

Figure 2.3.6.2 22 May 1972 plasma and field data. 
$0150 \quad 0200$

| 1

0210

0220

| 1

1

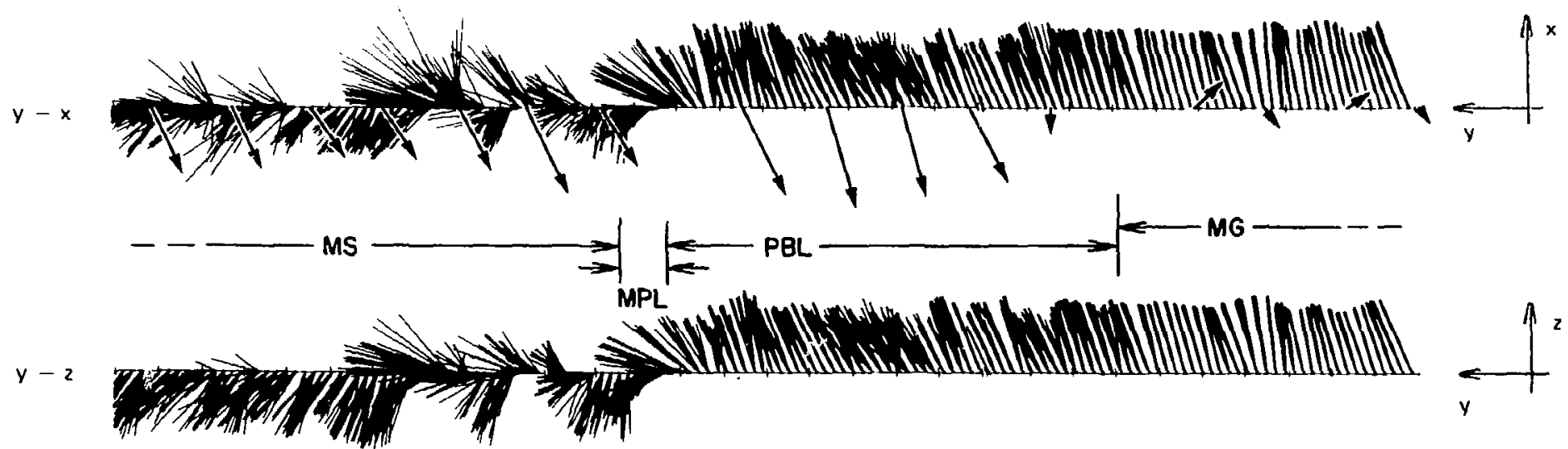

Figure 2.3.6.3 22 May 1972 plasma flow and field projections. 

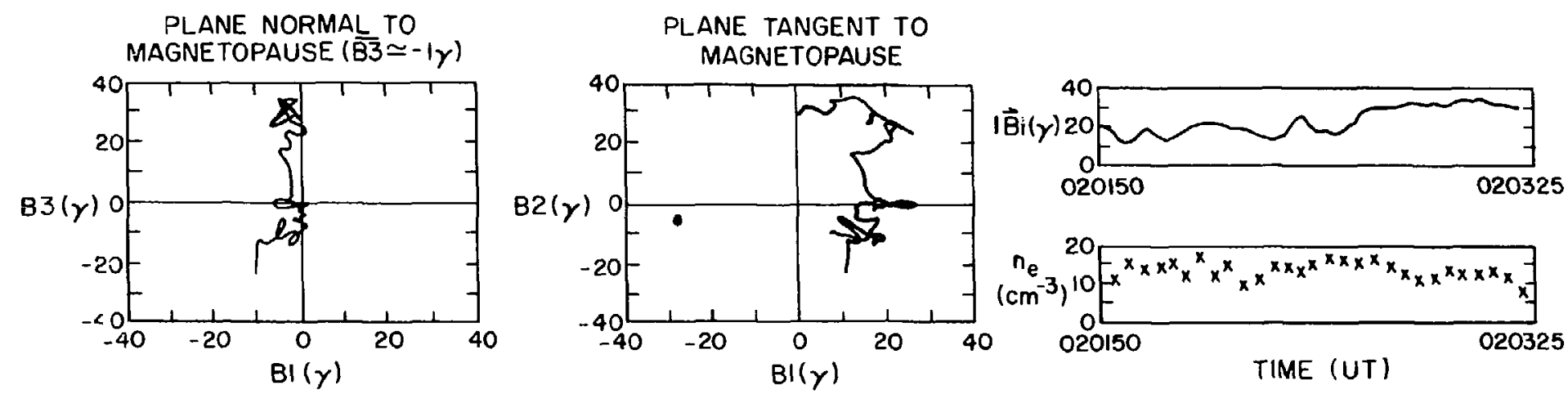

Figure 2.3.6.4 Maznetia field nojograms, field magnitude and electron density ror the 22 May 1972 erossing of the MPL. 
IONS
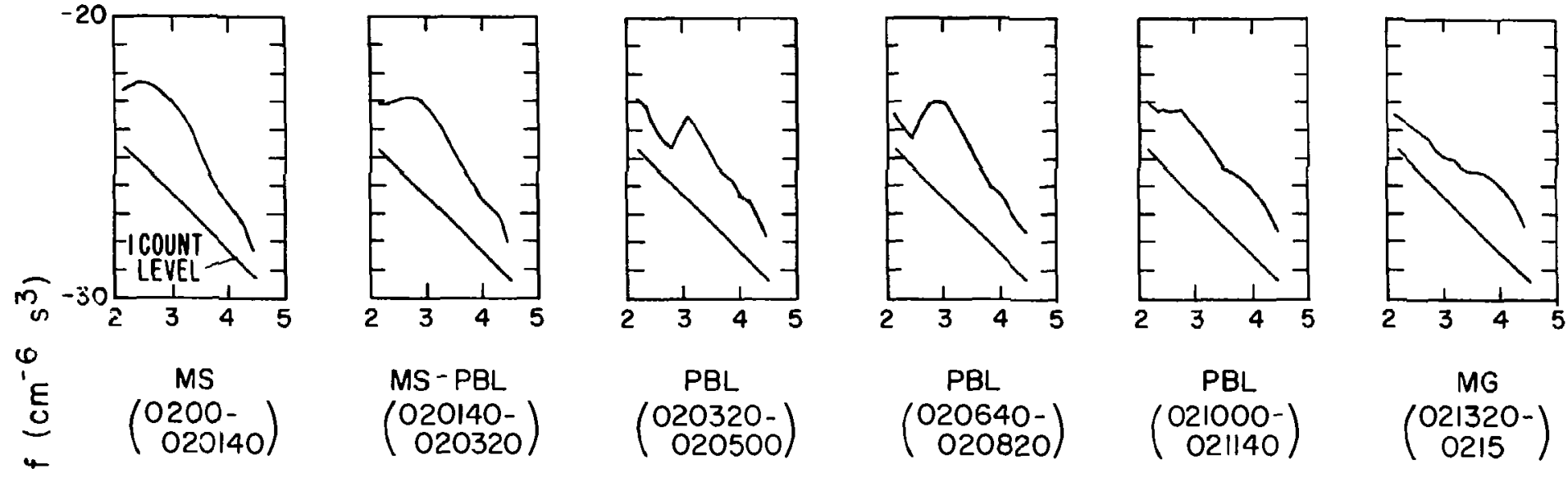

MS - PBL

$\left(\begin{array}{r}020140- \\ 020320\end{array}\right)$

PBL

$\left(\begin{array}{c}020320- \\ 020500\end{array}\right)$

PBL

$\left(\begin{array}{r}020640- \\ 020820\end{array}\right)$

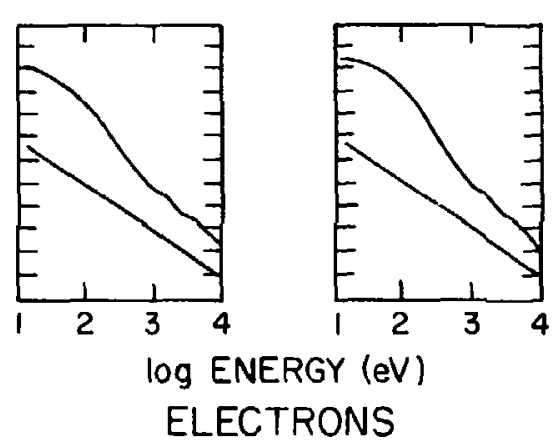

PBL

$\left(\begin{array}{c}021000- \\ 021140\end{array}\right)$

MG

$\left(\begin{array}{c}021320- \\ 0215\end{array}\right)$
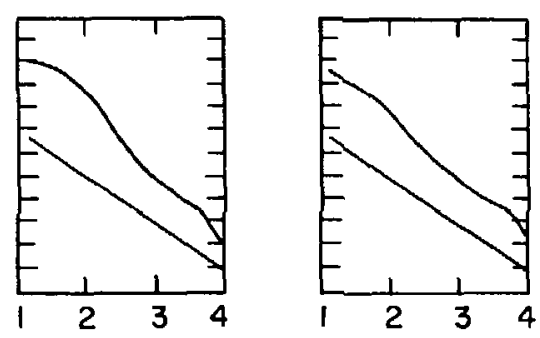

Figure 2.3.6.5 Az imuthal averages of the distribution function plotted for $\sim 100 \mathrm{~s}$ intervals for the 22 May 1972 crossing. 
indistinguishable from the nearby magnetosheath plasma, as shown in Figure 2.3.6.6. For eight spin periods spanning the MPL, electron differential spectra remain unchanged, which indicates relatively local entry of PBL plasma close to the MPL. The magnetospheric spectra have a lower flux at low energies and show a higher mean energy than spectra sampled near the MPL. 

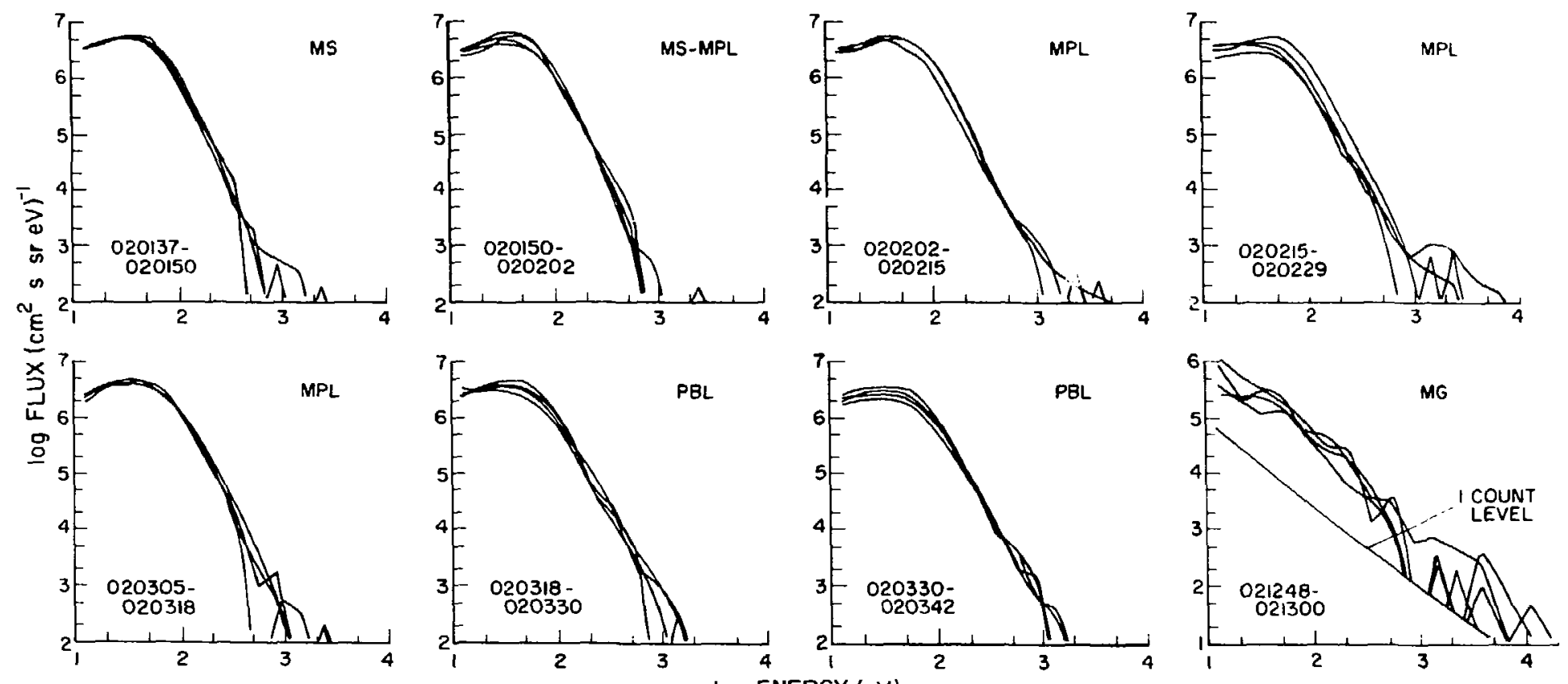

Figure 2.3.6.6 Electron differential spectra plotted four times every $\sim 12.5 \mathrm{~s}$ at successive locations near the MPL for the 22 May 1972 crossing. Nearby magnetospheric spectra are also plotted for comparison. 


\subsubsection{March 1972 IMP 6 Crossing}

This high-latitude, dawn-side IMP 6 crossing (Figures $2.3 \cdot 7,1-3$ ) shows field-aligned plasma flow directed into the cusp region. The flow is also enhanced by $\wedge 100 \mathrm{~km} / \mathrm{s}$ in speed relative to the nearby magnetosheath plasma. A minimum variance analysis of the magnetic field indicates a normal field component of $8 \mathrm{r}$, as shown by the hodograms in Figure 2.3.7.4. There is a local minimum of electron density and field magnitude withir, the MPL as shown on the right-hand plot of Figure 2.3.7.4. The plasma flow direction shifts suddenly near the MPL into a direction that is nearly field-aligned within the PBL, as shown in Figure 2.3.7.3. Such field-aligned flow is common in the cusp region (c.f., the 22 May 1972 crossing).

The plasma and field parameters observed in the PBL suggest that magnetosheath plasma has crossed the MPL on the subsolar side of this crossing location and is flowing into the cusp region. This cuspwarddirected flow may be related to the earthward-directed flows observed in the cusp by IMP 6 at smaller radial distances (Fairfield and Hones, 1978).

Energetic electron pitch angle distributions, shown in Figure 2.3.7.5, show a pancake-shaped distribution in the PBL and the nearby magnetosphere, whereas the nearby magnetosheath shows a streaming distribution directed away from the cusp region. The pitch-angle distributions as well as the energetic electron intensities are clearly different on each side of the MPL. These results suggest that the PBL is on closed field lines and that part of the energetic electron 


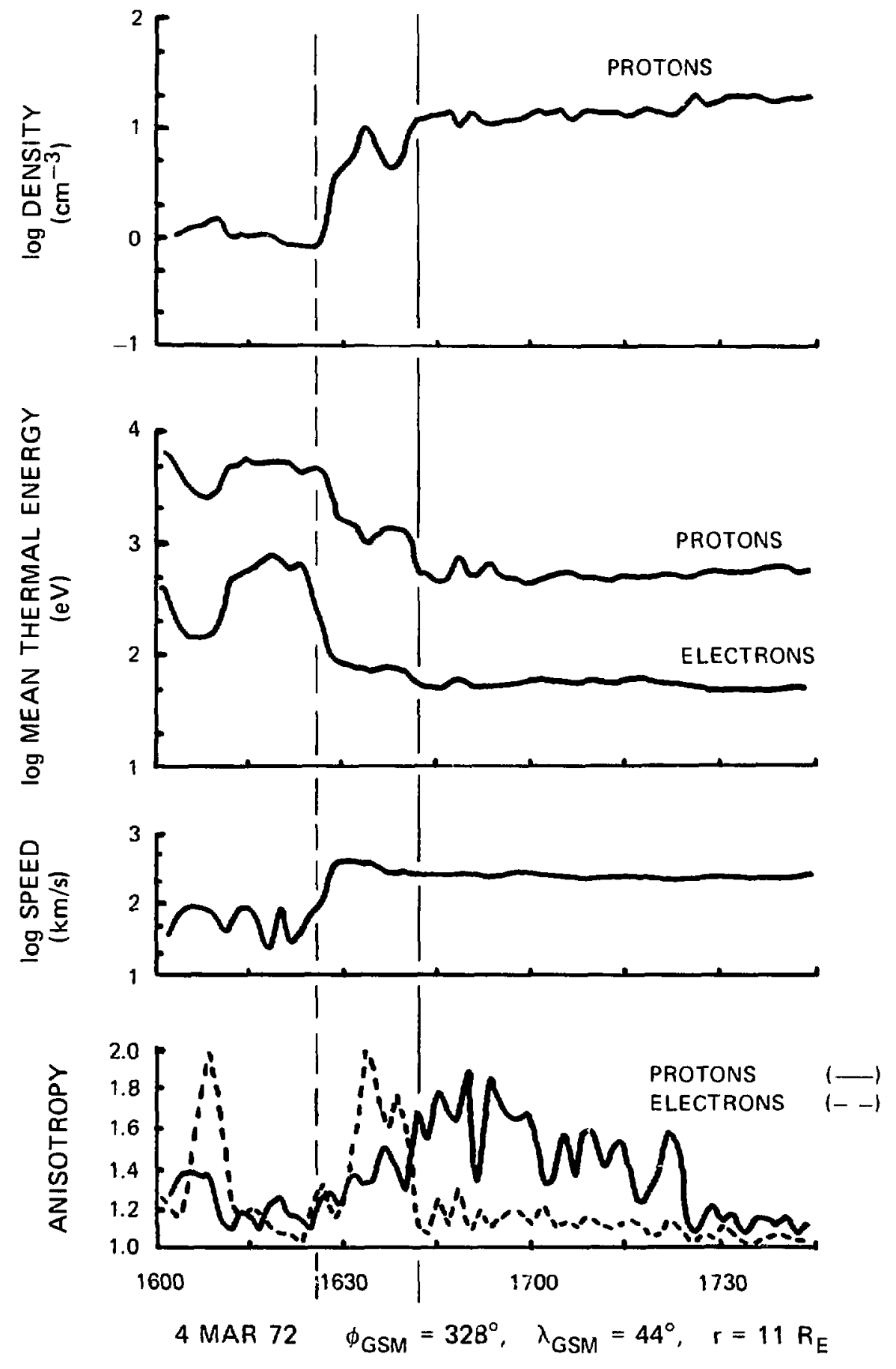

Figure 2.3.7.1 4 March 1972 plasma iata. 


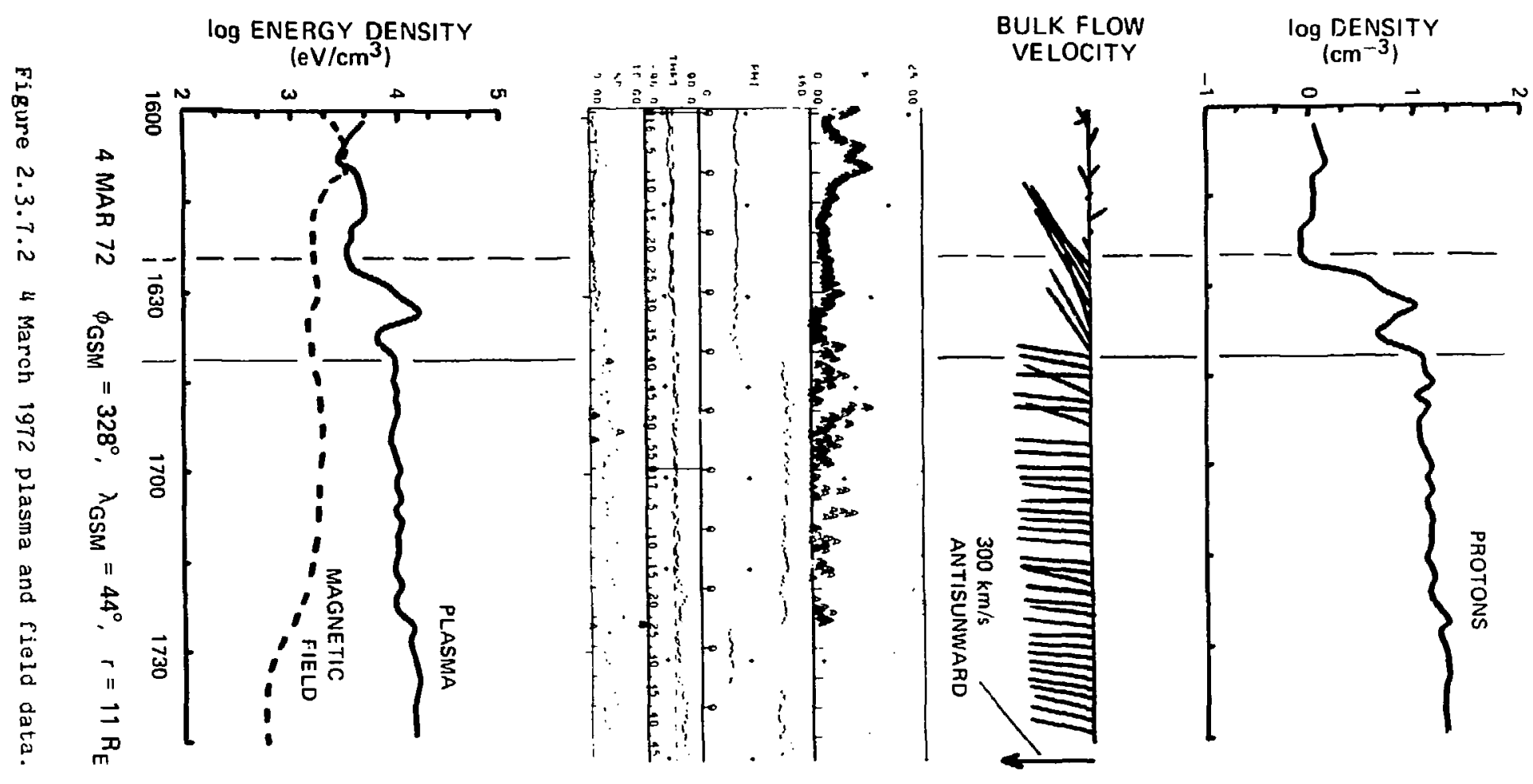




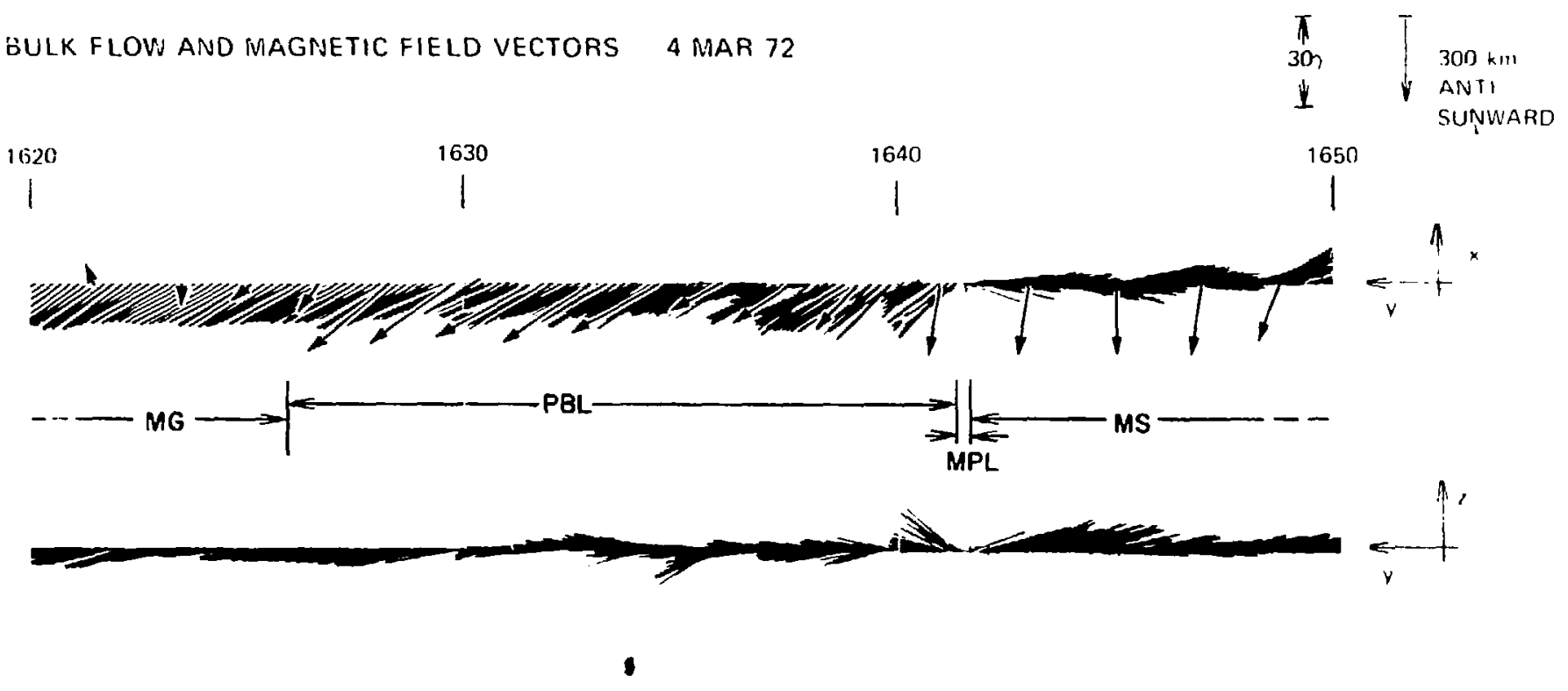

Figure 2.3.7.3 4 March 1372 plasma flow and field projections. 


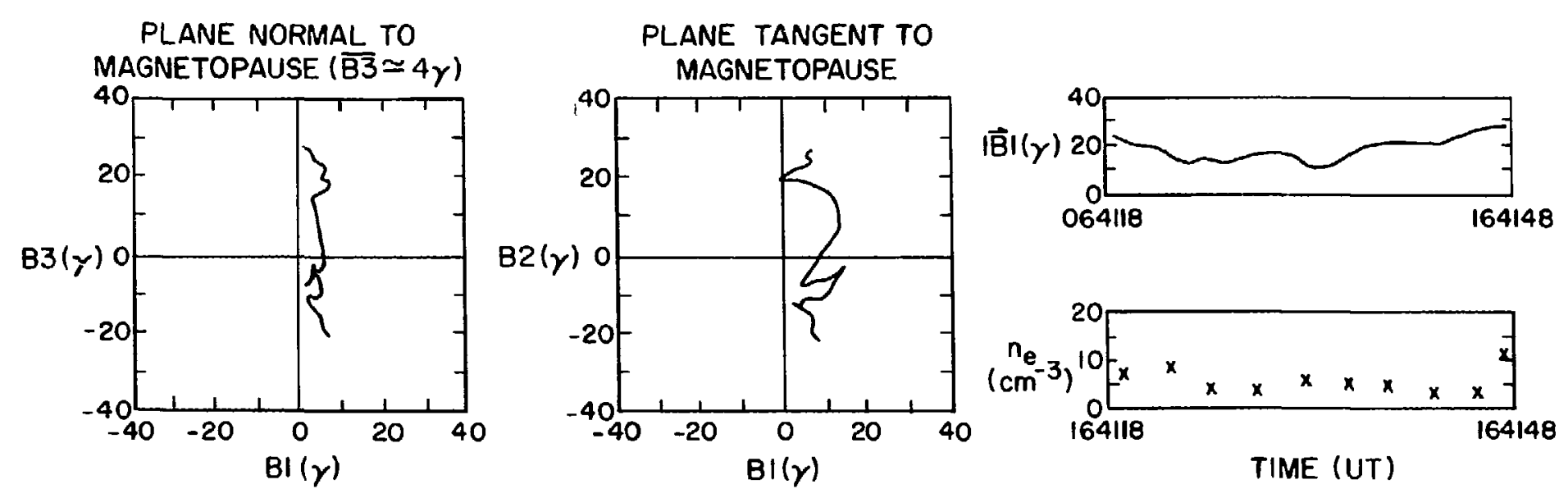

Figure 2.3.7.4 Magnetic field hodograms, field magnitude and electron density for the 4 March 1972 crossing of the MPL. 


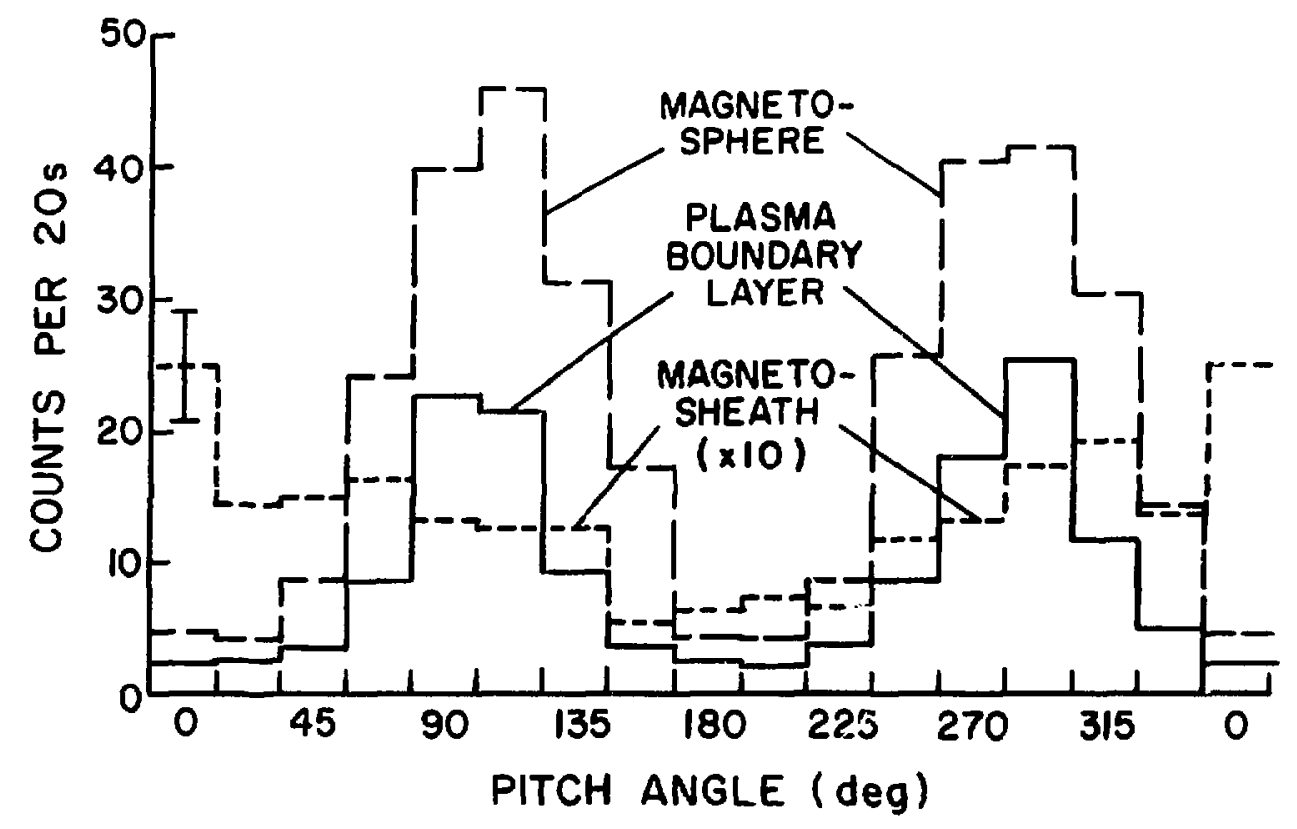

Figure 2.3.7.5 Energetic electron (47- to 350-keV) pitch-angle distributions for the 4 March 1972 crossing. 
population can cross the MPL and contribute to a layer of energetic electrons in the magnetosheath adjacent to the MPL.

Electron differential energy spectra, (Figure 2.3.7.6) show the same close similarity of PBL and magnetosheath spectra as is usually observed, as well as the usual contrast with the nearby magnetospheric spectra. At a local density minimum near 1638 UT, however, the energy spectra are close to those observed in the nearby magnetosphere in the same region where a local increase in mean thermal energy is observed. These observations are consistent with a mixing of magnetosheath and magnetospheric plasma in the PBL. Azimuthal averages of the ion and electron velocity distributions are presented in Figure 2.3.7.7. The magnetosheath and $\mathrm{PBL}$ spectra are very closely related and are mutually different from the nearby magnetospheric spectra. Although the energetic electron pitch angle distributions change dramatically across the MPL, the low-energy plasma spectra show a distinctive continuity across the MPL. 

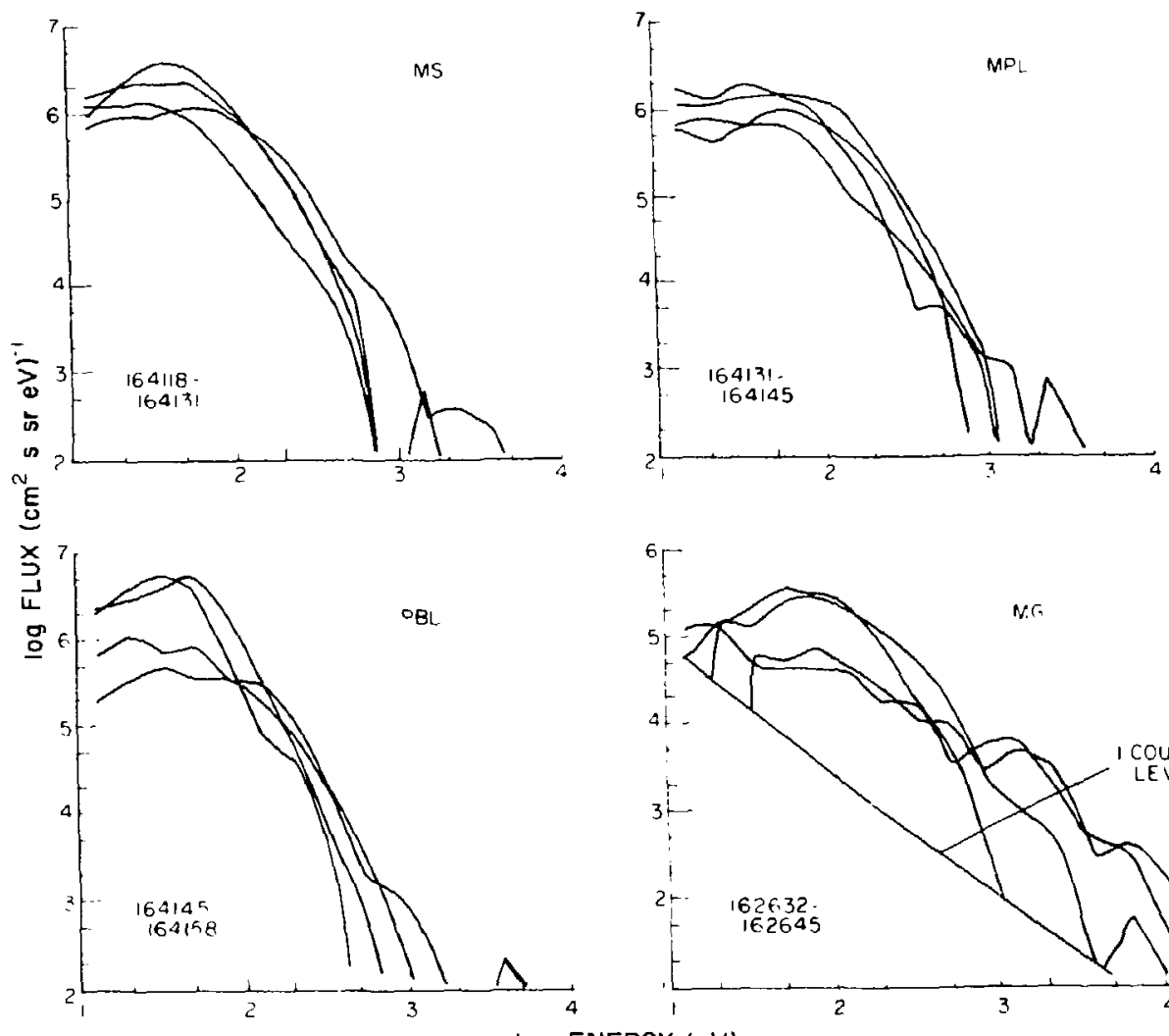

log ENERGY (eV)

Figure 2.3.7.6 Electron differential spectra plotted four times every

$-12.5 \mathrm{~s}$ at successive locations near the MPL for the 4 March 1972

crossing. Nearby magnetuspheric spectra are also plotted for comparison. 
i

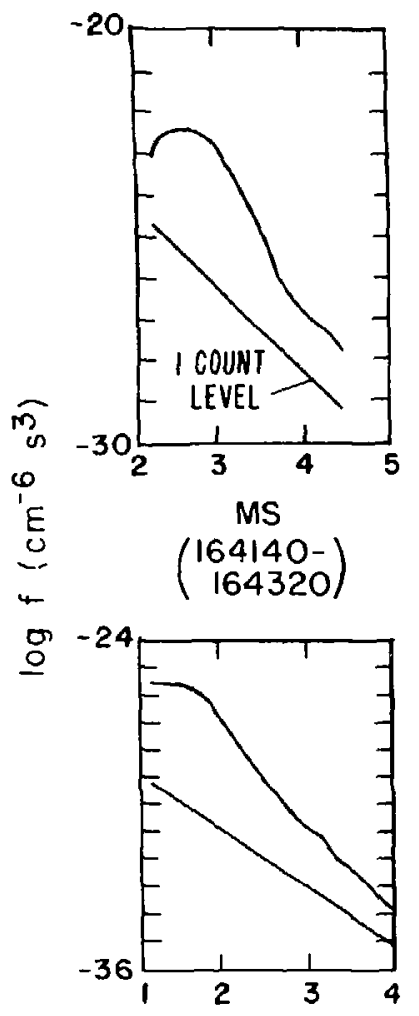

IONS
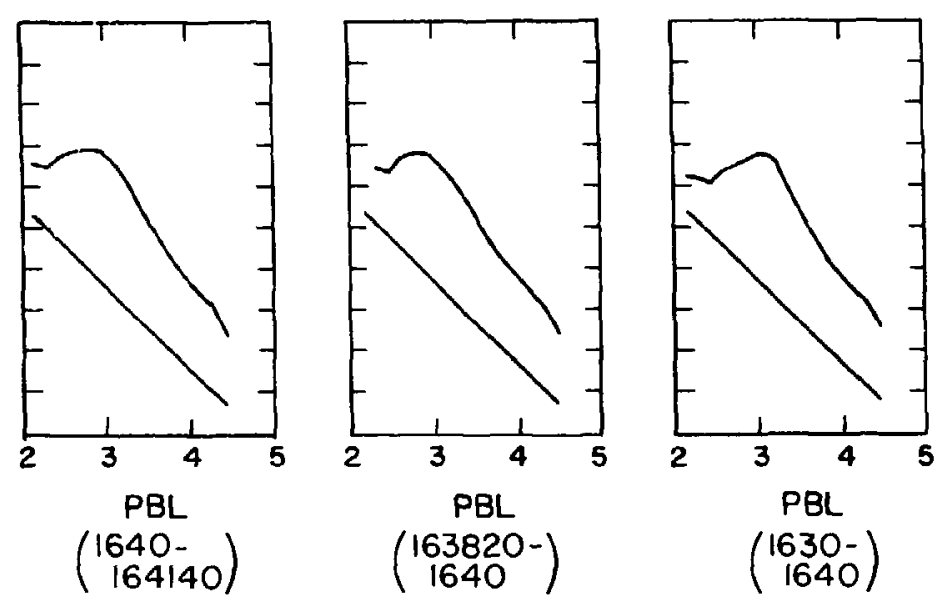

$(1630-$

$\left.\begin{array}{r}1630- \\ 1640\end{array}\right)$
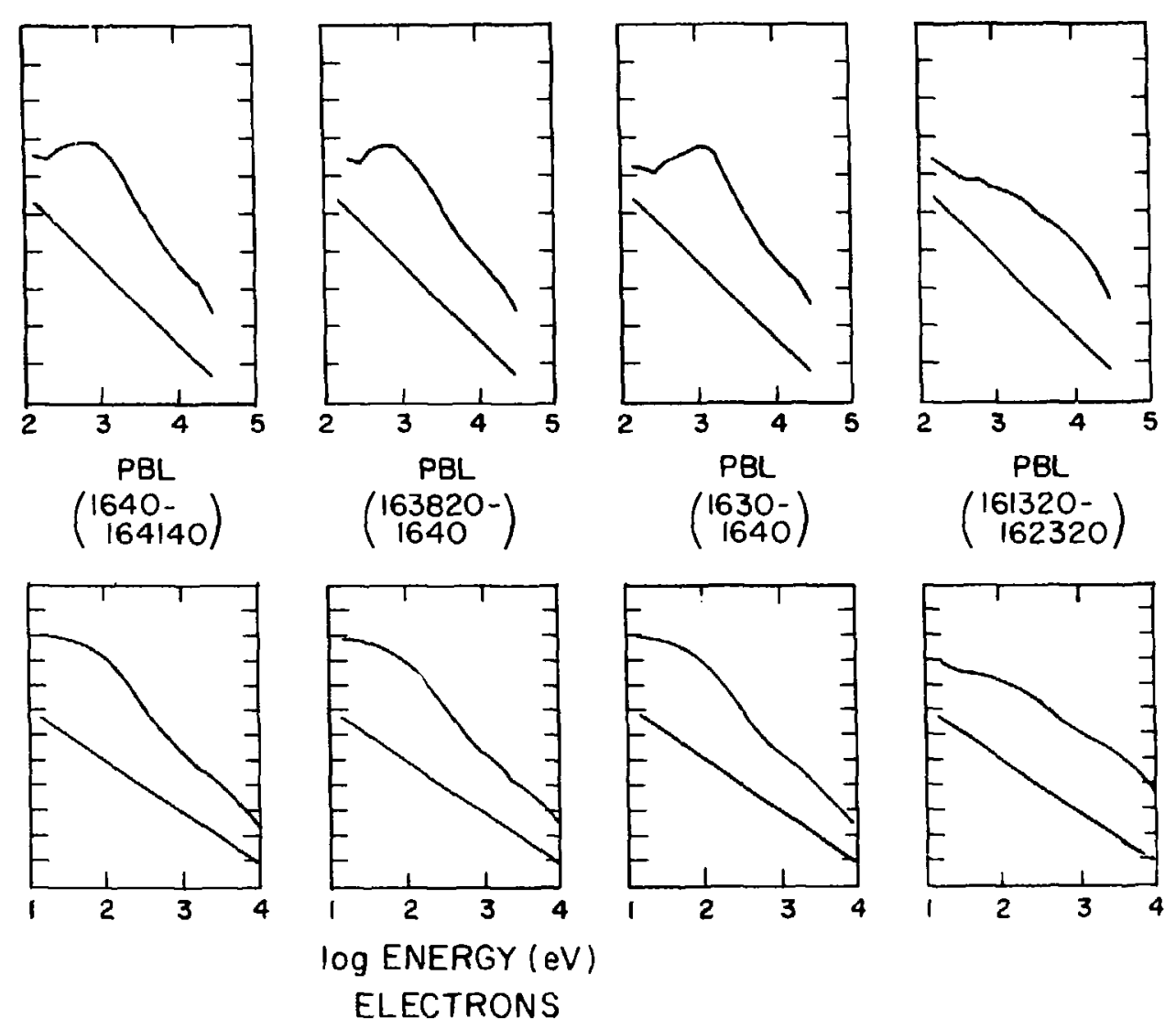

$\log \operatorname{ENERGY}(\mathrm{eV})$

ELECTRONS

Figure 2.3.7.7 Azimuthal averages of the distribution function plotted for $\sim 100 \mathrm{~s}$ intervals for the 4 March 1972 crossing. 


\subsubsection{ISEE Dual Satellite Crossings}

Haerendel et al. (1978) report that a plateau in derisity values is often observed in the PBL as seen by HEOS 2. A plateau in density is marked by a sudden density change at the magnetopause layer and at the inner extent of the PBL. An ISEE 1 crossing on 3 November 1977 (Paschmann et al., 1978) that shows a plateau signature is shown in Figure 2.3.8.1 (captions face Figures 2.3.8.1-2). This signature is rarely observed by IMP 6; instead, the majority of IMP 6 crossings reveal no distinct changes in density or electron spectra at the magnetopause layer. The ISEE observations suggest a spatial change in PBL structure with increasing distance from the subsolar region when compared to the IMP 6 observations. This is further emphasized in the ISEE 2 crossing of 8 November 1977 (Paschmann et al., 1978), shown in Figure 2.3.8.2. This crossing shows a PBL detached from the magnetopause layer, a situation that has not yet been clearly found in the IMP 6 data. The isolated PEL in this ISEE 2 crossing has magnetosheath-like spectra (Paschmann et al., 1978), although it is located on magnetospheric field lines (Russell and Elphic, 1978). Paschmann et al. (1978) describe a possible temporal model for these isolated PBL regions in which "plasma entry and/or transport along the boundary are 'switched' on and off." The flow parameters shown in Figure 2.3.8.2 during the detached PBL segment show a dawn-dusk flow reversal that suggests a vortex of plasma flow. Such possible vortices are evident in other segments of the ISEE PBL crossings near the noon meridian at $25^{\circ}$ latitude. Paschmann et al. (1978) point out that the apparent PBL thickness may be produced by "temporally limited plasma entry" and/or the passage of plasma vortices. 
Figure 2.3.8.1 Two- and three-dimensional plasma and field parameters for the ISEE 1 crossing of 3 November 1977. Solar magnetospheric (GSM) coordinates are used for spacecraft positions with $R$ (in $R_{E}$ ), local time (LT in hours), and latitude (in degrees). Solid lines denote protons and dotted lines denote electrons in the plots of density ( $N$ in $\mathrm{cm}^{-3}$ ) and temperature ( $T$ in degrees Kelvin). Bulk flow directions are given in terms of azimuth $\left(\phi_{p}\right)$ and elevation $\left(\Lambda_{p}\right)$ in spacecraft coordinates (close to solar ecliptic coordinates) based on both the 2D (solid line) and 3D (dots) moment analysis. $M, E, N$, or $S$ denote morning, evening, northward, or southward flow components, respectively. Total plasma pressure (solid line) and magnetic field pressure (dotted line) are given in units of $10^{-8}$ dynes $/ \mathrm{cm}^{2}$ (left scale). The dotted curve also gives the magnetic field strength (in gammas) by using the quadratic right-hand scale. The magnetopause is marked by a solid vertical line and the inner surface of the plasma boundary layer is marked by a dashed line. Magnetosheath plasma is sampled before 0751 UT and magnetospheric plasma is sampled after 0803 UT. 


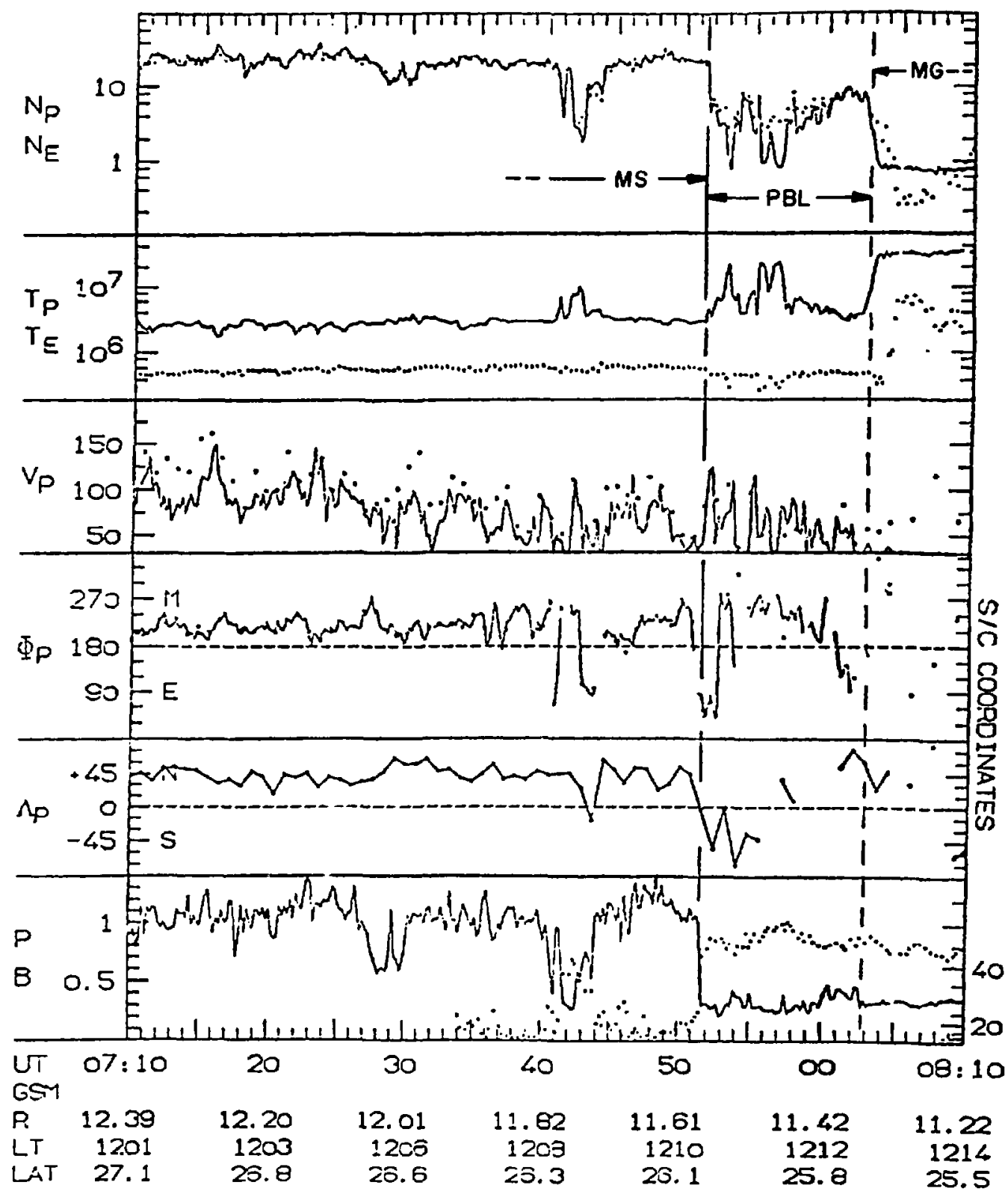


Flgure 2.3.8.2 Two- and three-dimensional plasma and fleld parameters for the ISEE 2 crossing of 8 November 1977. This figure uses the same format as Figure 2.3.8.1 except that an isolated plasma boundary layer 1s marked off by vertical dashed 11nes at 0254:50 and 0258 UT. The magnetopause layer is centered on the vertical solid line at 0252 UT. 


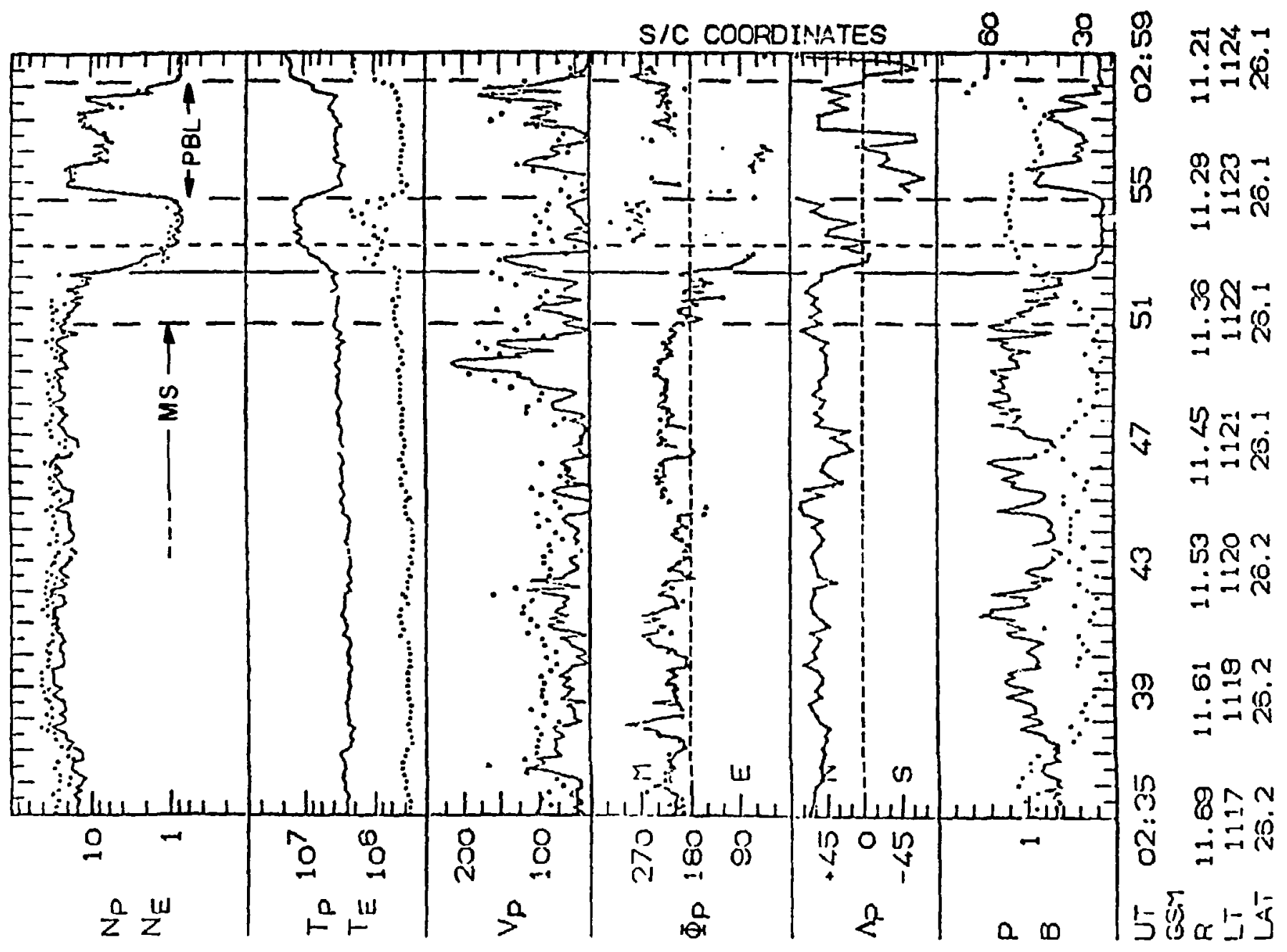


A comparison of the ISEE and IMP 6 results shows that the PBL structure within $\backsim 30^{\circ}$ of the subsolar point is probably different from its structure along the flanks farther from the subsolar region. The relationship among plasma flow observations by IMP 6 , HEOS 2 and the ISEE satellites is evident when flow directions for various crossings are compared (see section 2.4.3). 


\subsection{Statistical Study}

\subsubsection{Basic Questions}

Because of the substantial spatial and temporal variations of plasma and field parameters observed near the magnetospheric boundary, several important observational questions can be answered only by evaluating data from satellite crossings in a collective manner. Some questions important for determining the primary source of PBL plasma are

(1) How do plasma densities and energy spectra usually vary near the MPL and across the PBL?

(2) What are typical bulk plasma flow characteristics in the PBL and nearby magnetosheath?

(3) What are nominal thicknesses of the PBL, how thick is the PBL relative to the $\mathrm{MPL}$, and how does nominal $\mathrm{PBL}$ thickness vary with the local z-component of the magnetosheath magnetic field and other parameters?

(4) What are typical characteristics of energetic electrons near the MPL and PBL?

\subsubsection{Density Variations}

Figure 2.4.2.1 shows observed variations of electron density samples in high time resolution (time between samples is $\$ 12.5 \mathrm{~s}$ unless noted by $\Delta T$ ) in the MPL and PBL for eight IMP 6 crossings. For comparison, every crossing is plotted to show the same apparent PBL width. The MPL is often not distinguished by large changes in plasma density. In fact, local electron densities and energy spectra, sampled every $n 3.1 \mathrm{~s}$ by IMP 6, showed no distinct changes at the MPL in 24 of 40 selected crossings. Three of the seven most rapid crossings that have been 


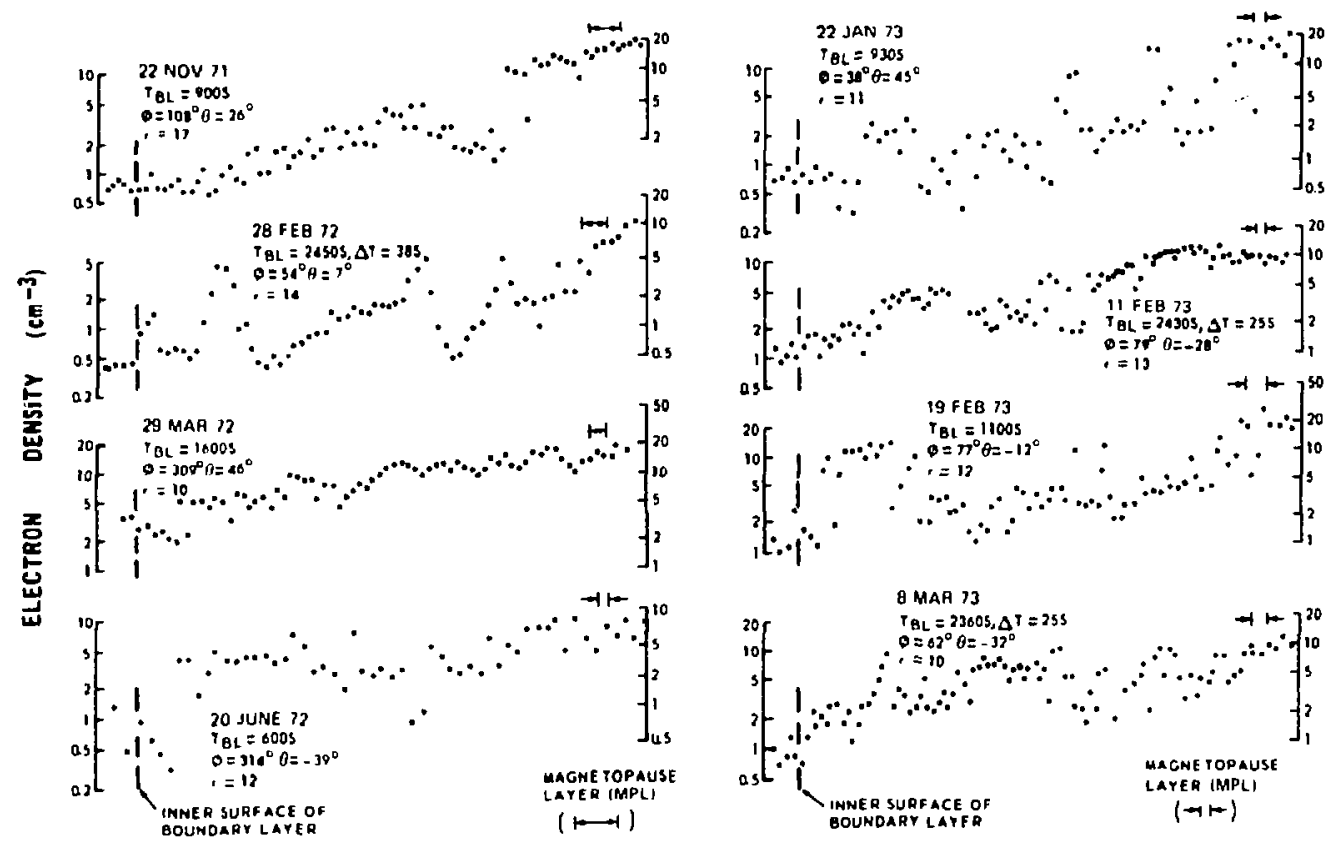

Figure 2.4.2.1 Electron density data are plotted for selected PBL crossings characterized by a single, well-defined MPL. Satellite time in the $P B L$ is denoted by $T_{B L}$. Data points that give averages of three-second electron density samples are separated by $\cong 12.5 \mathrm{~s}$ time incervals unless noted by $\triangle T$. The PBL is given the same apparent wisth in all plots with the MPL positioned on the right-hand side. All locations are given in GSM coordinates. 
identified also showed no distinct changes in electron density or energy spectra at the MPL. These crossings show energy spectra within the PBL that are often virtually indistinguishable from those of the nearby magnetosheath. In addition, electron spectra measured through the MPL are much more similar to each other than electron spectra compared between different crossings (e.g., compare Figures 2.3.5.6 and 2.3.7.6). This spectral similarity of plasma on opposite sides of the MPL with a general gradation toward. magnetospheric spectra farther into the PBL (especially during periods of relative density minima) has also been noted in data from HEOS 2 (e.g., the 10 October 1973 crossing, Haerendel et al., 1978).

In 40 crossings selected for examination in the highest possible time resolution, the basic PBL signatures were never found to be completely absent. This set includes the seven most rapid IMP 6 crossings where the nominal PBL thickness was as small as one ion gyroradius (typically $\backsim 100 \mathrm{~km}$ for protons near the magnetopause).

Significant density fluctuations are observed by IMP 6 in the PBL-MPL region (Figure 2.4.2.1) as well as the nearby magnetosheath (Figure 2.4.2.2). Changes in electron density greater than a factor of two are often observed for two consecutive (3-s interval) samples in these regions, especially in the PBL. These variations indicate the presence of significant small-scale inhomogeneities in the PBL and nearby magnetosheath. Single satellite measurements can provide only a rough estimate of gradient scale lengths (see Table 3.1.1) since the flow field is sampled only locally. Density fluctuations in the PBL-MPL 


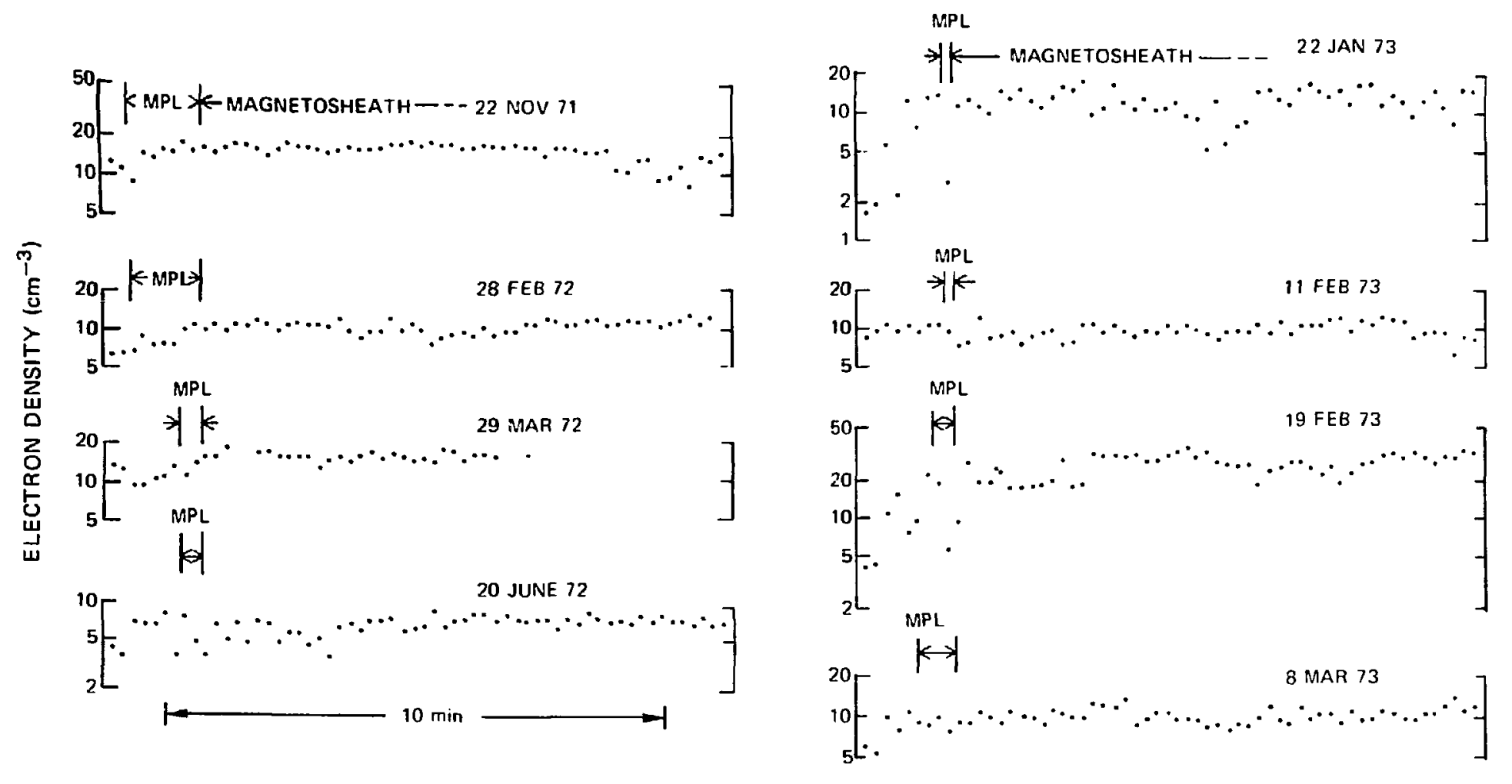

Figure 2.4.2.2 Electron density values sampled in the magnetosheath adjacent to the MPL-PBL regions shown in Figure 2.4.2.1. Density values are plotted every-12.5 s (i.e., averaged over four electron spectra sampled during each spin period) at equal time spacing for all crossings. 
region could lead to cross-field plasma diffusion because of plasma kinetic instabilities (see section 3.3 ).

2.4.3 Bulk Plasma Flow Characteristics

The spatial distribution of source(s) for the PBL should be reflected in PBL bulk flow characteristics, which are reviewed in Figure 2.4.3. Flow vectors are drawn based on IMP 6, HEOS 2 (Rosenbauer et al., 1975) and ISEE observations (Paschmann et al,, 1978). All flow vectors drawn in the $\mathrm{PBL}$ in the equatorial plane or noon-midnight meridian plane are based on projecting observed flow vectors for crossings that occurred within $15^{\circ}$ of the GSM X-Y or X-Z plane, respectively. Other flow vectors are estimated projections of observed flow vectors projected onto the magnetopause surface. Measurements by IMP 6 near the noon meridian and in the cusp are represented by flow vectors that are assumed to be aligned with the general field configuration shown in Figure 2.4.3. Since IMF 6 measures an effective two-dimensional velocity distribution centered on the ecliptic plane (see section 2.2 and Appendix $A$ ), flow vectors marked in the $x-z$ plane represent only speed and the $x$-component of flow. Flow components out of the ecliptic are not measured by IMP 6 although they are usually measured by HEOS 2 . Substantial changes in plasma flow directions are frequently observed in the subsolar region (as sampled by ISEE) and the cusp regions. The high-latitude boundary layer (HLBL) and lnw-latitude boundary layer (LLBL), see Figure 1.1, consistently show antisunward flow. The LLBL plasma flow often has a significant cross-field component, especially near the magnetospheric equatorial plane and in the subsolar region. Antisunward component flow observed in the near-noon 


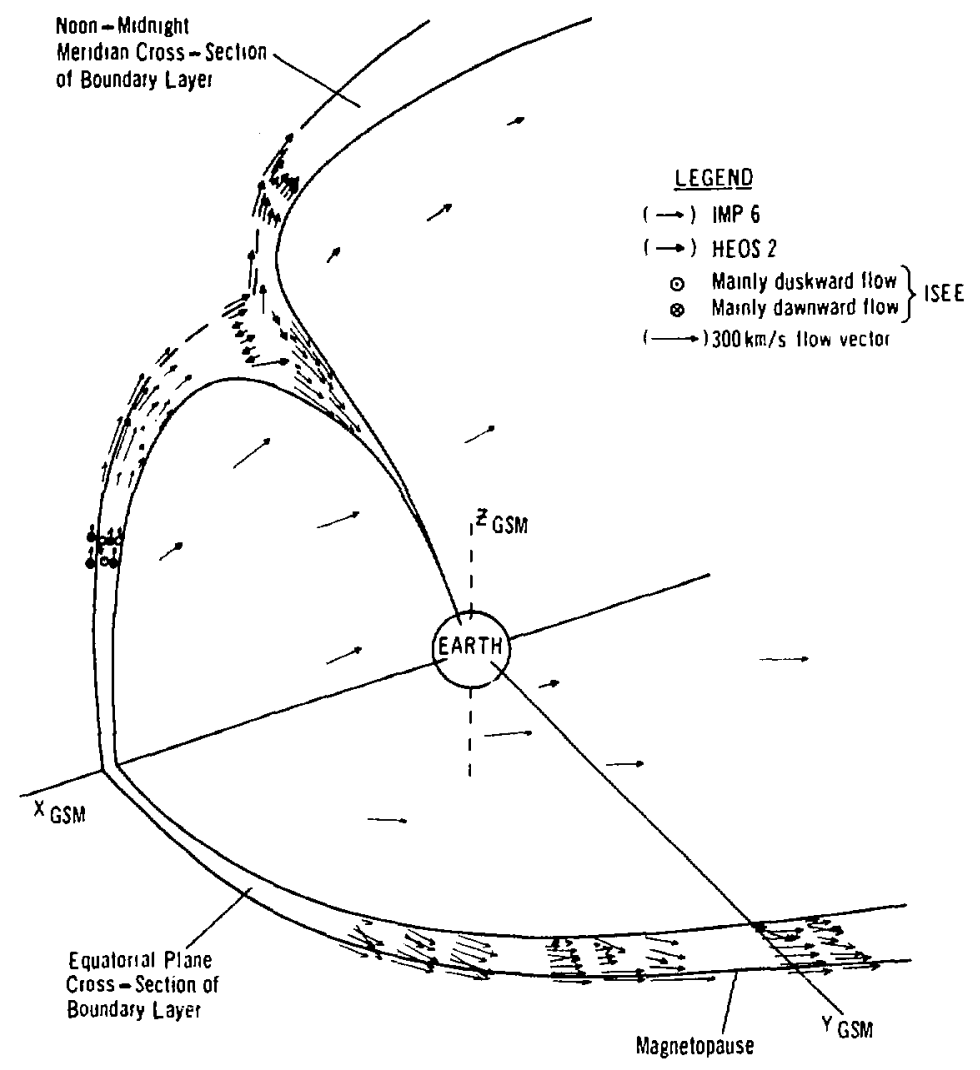

Figure 2.4.3 Summary of plasma flow in the sunwar 3 plasma boundary layer as observed by IMP 6, HEOS 2, ISEE 1 and ISEE 2. Approximate projections of observed flow vectors are given on the noon-midnight meridian and equatorial plane cross-sections of the PBL based on satellite crossings with an earth centered angle of $<15^{\circ}$ from the $X-Z$ and $X-Y$ planes, respectively. Flow vectors marked between the two cross-sections are observed PBJ. flow vectors projected onto the magnetopause surface. The magnetosheath bulk flow adjacent to the magnetopause was assumed to be parallel to the magnetopause surface for this plot. This drawing has been made to scale except that the $\mathrm{PBL}$ is enlarged $\sim 50 \%$. 
meridian PBL, equatorward of the cusp regions, is not consistent with sources limited solely to the cusp regions.

The IMP 6 measurements indicate that the FBL is supplied by direct transport of magnetosheath plasma across the MPL and that this transport process is relatively widespread over the entire sunward magnetospheric bouncary. IMP 6 observations, including that of plasma flow, show no evidence that the PBL can be filled only by magnetosheath plasma entering the cusp regions or by highly localized sources in the subsolar region.

\subsubsection{PBL-MPL Thickness Ratio}

The effect that is most clearly incompatible with classical diffusion (see section 3.3) is the frequent observation that the $\mathrm{PBL}$ thickness $\mathrm{L}_{\mathrm{PBL}}$ is usually substantially larger than the current layer thickness $\mathrm{L}_{\mathrm{MPL}}$ - Table 2.4 .4 lists estimated $\mathrm{L}_{\mathrm{PBL}} / \mathrm{L}_{\mathrm{MPL}}$ ratios. These ratios are independent of uniform magnetopause motions. For nine selected crossings at low latitudes $\left(\left|\theta_{\mathrm{GSM}}\right| \leq 30^{\circ}\right)$ that show a clear MPL signature, the mean $L_{P B L} / L_{M P L}$ ratio was 18.2 , with values ranging from 4 to 74 with a standard deviation of the mean of 22 . This result indicates that the PBL thickness is highly variable and, in generai, $\mathrm{L}_{\mathrm{PBL}}$ is significantly larger than $\mathrm{L}_{\mathrm{MPL}}$. If we take one ion gyroadius ( $*$ $140 \mathrm{~km}$ for protons), as the approximate MPL thickness (as expected for a Parker magnetopause, see section 3.2), then a typical thickness of the FBL is $\sim 0.4 R_{E}$. 
Table 2.4.4 PBL versus MPL Thickness

\begin{tabular}{|c|c|c|c|}
\hline Date & MPL Interval (s) & PBL Interval (s) & $\begin{array}{l}\text { Estimated } \\
\mathrm{L}_{\mathrm{PBL}} / \mathrm{L}_{\mathrm{MPL}}\end{array}$ \\
\hline 22 November 1971 & 93 & 850 & 9.1 \\
\hline 5 January 1972 & 60 & 1230 & 20.5 \\
\hline 4 February 1972 & 60 & 360 & 6.0 \\
\hline 28 February 1972 & 88 & 680 & 7.7 \\
\hline 4 March 1972 & 15 & 890 & 59.3 \\
\hline 24 March 1972 & 72 & 260 & 3.6 \\
\hline 14 April 1972 & 24 & 1780 & 74.2 \\
\hline 13 May 1972 & 13 & 165 & 12.7 \\
\hline 22 May 1972 & 85 & 550 & 6.5 \\
\hline 20 June 1972 & 12 & 546 & 45.5 \\
\hline 22 January 1973 & 12 & 906 & 75.5 \\
\hline 16 February 1973 & 3 & 156 & 52.0 \\
\hline 19 February 1973 & 45 & 975 & 21.7 \\
\hline 1 May 1973 & 48 & 506 & 10.5 \\
\hline 16 December 1973 & 100 & 1020 & 10.2 \\
\hline 24 December 1973 & 90 & 960 & 10.7 \\
\hline
\end{tabular}




\subsubsection{PBL Thickness Estimates}

IMP 6 plasma experiments provide good coverage of the dayside magnetopause, as shown in Figure 2.2.2, except within $\sim 25^{\circ}$ of the subsolar point. From a larger set of 90 well-defined, nonmultiple crossings, we have evaluated the nominal thickness of the low-latitude portion of the PBL (or LLBL) by projecting the spacecraft trajectory in each boundary layer segment onto a local normal to the magnetopause surface based on the model of Howe and Binsack (1972). Table 2.4.5 lists these nominal thickness values versus $X_{G S M}$ (distance along the earth-sun axis) along with their mean values. Nominal thickness values are expected to vary considerably because of the unknown magnetopause motion relative to the spacecraft. Table 2.4 .5 also shows that the mean LLBL thickness tends to increase with increasing distance from the subsolar region (i.e., with decreasing $x_{G S M}$ ). A local increase in mean thickness for $X_{G S M}=4$ to $5 R_{E}$ is due to the inclusion of some crossings near the cusp regions at high latitudes, although the IMP 6 orbit rarely provides a passage directly into the cusp. A few cases of IMP 6 crossings in the cusp region are discussed in detail by Fairfield and Hones (1978).

From the set of 90 crossings used here to evaluate the nominal boundary layer thickness, no statistically significant correlation has been found between LLBL thickness and latitude (except for the cusp regions where the PBL tends to be thicker), geomagnetic activity, or with the locally measured z-component of the magnetosheath magnetic field. Figure 2.4.5.1 shows the PBL thickness values plotted versus the 
Table 2.4.5. Nominal Plasma Boundary Layer Thickness Values and their Means Versus $\mathrm{X}_{\text {GSM }}$

\begin{tabular}{|c|c|c|}
\hline$X_{G S M}\left(R_{E}\right)$ & Boundary Layer Thickness, $L_{P B L}\left(R_{E}\right)$ & L \\
\hline $0-1$ & $0.35,0.44,0.16,0.06,0.66$ & 0.35 \\
\hline $1-2$ & $0.33,0.74,0.39,0.46,1.02,0.12,0.83$ & 0.56 \\
\hline $2-3$ & $0.42,0.14,0.40,1.2,0.20,0.08,1.1$ & 0.51 \\
\hline $3-4$ & $\begin{array}{l}0.33,0.13,0.02,0.50,1.29, .012,0.07,0.08,0.19,0.61, \\
0.59,0.33\end{array}$ & 0.36 \\
\hline $4-5$ & $\begin{array}{l}0.60,1.32,0.37,1.36,0.45,1.47,0.04,0.48,0.40,0.47,0.27, \\
0.42,0.14\end{array}$ & 0.60 \\
\hline $5-6$ & $\begin{array}{l}0.41,0.98,0.56,0.26,0.12,0.16,0.08,0.28,0.32,0.24, \\
0.17,0.52\end{array}$ & 0.34 \\
\hline $6-7$ & $0.93,0.21,0.48,0.16,0.07,0.10,0.09,0.05,0.30$ & 0.25 \\
\hline $7-8$ & $\begin{array}{l}0.10,0.15,0.05,0.004,0.04,0.06,0.05,0.02,0.04,0.07, \\
0.03,0.43,0.09,0.04\end{array}$ & 0.08 \\
\hline $8-9$ & $0.16,0.13,0.03,0.003,0.005,0.36$ & 0.11 \\
\hline $9-12$ & $0.01,0.001,0.005,0.23$ & \\
\hline
\end{tabular}




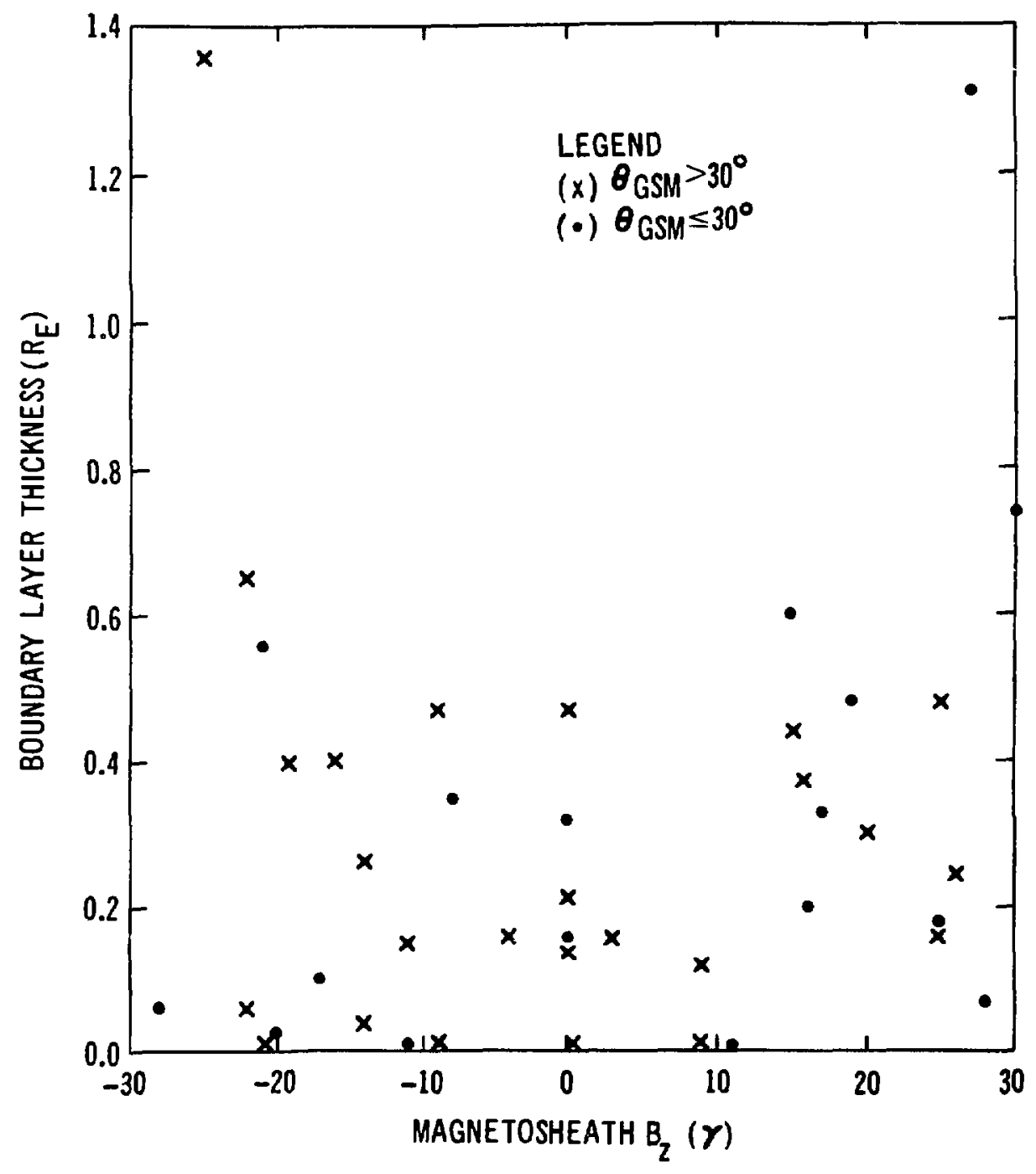

Figure 2.4.5.1 Plasma boundary layer thickness values are plotted versus the local 2 component of the magnetic fleld, $B_{2}$, as measured by IMP 6 in the magnetosheath adjacent to the magnetopause. From the larger set of 90 crossings, only those crossings which permitted a rough estimate of $B_{2}$ were used. 
local z-component of the magnetic field measured by IMP 6 in the magnetosheath region adjacent to the magnetopause. Crossings for which $\left|\theta_{\text {GSM }}\right|$ is greater than $30^{\circ}$ are separated in this figure which shows no significant correlation between nominal boundary layer thickness and the local z-component of the magnetosheath magnetic field. Space and/or time variations of actual boundary layer thickness might suppress any raal correlation. Such variations have been described by Williams (1978) who used ISEE measurements of three-dimensional distributions based on $24-44.5 \mathrm{keV}$ ions. These measurements were consistent with boundary motions toward and away from the earth of up to $20 \mathrm{~km} / \mathrm{s}$ along with surface waves on the boundary.

The LLBL thickness progressively increases with increasing distance from the subsolar point as shown in Figure 2.4.5.2b. Nominal thickness values near the dawn-dusk meridian plane $\left(X_{G S M}=0\right)$ are $s 0.5 R_{E}$. The increased thickness for $4<X\left(R_{E}\right) \leq 5$ shown in Figure 2.4.5.2e is due to enhanced PBL thickness at high latitudes near the cusp regions. Nominal thickness values are distributed over a wide range (up to 1.5 $R_{E}$ ) as shown in Figure 2.4.5.2a, with a mean value of $0.34 R_{E}$ and a standard deviation of the mean of $0.35 \mathrm{R}_{\mathrm{E}}$. The thickness distribution for negative versus positive IMF $B_{z}$ (or IMF $B_{y}$ ) as shown in Figures 2.4.5.2c,d shows that the frequency of a thin LLBL is reduced for negative IMF $B_{z}$ (or positive IMF $B_{y}$ ). HEOS 2 measurements have not shown any correlation between IMF $B_{z}$ and LLBL thickness (Haerendel et al., 1978) although they have shown a distinct correlation with HLBL thickness (Sokopke et al., 1976). PBL thicknesses for crossings tailward of the dawn-dusk meridian plane $\left(x_{G S M}=0\right)$ have lower mean values 

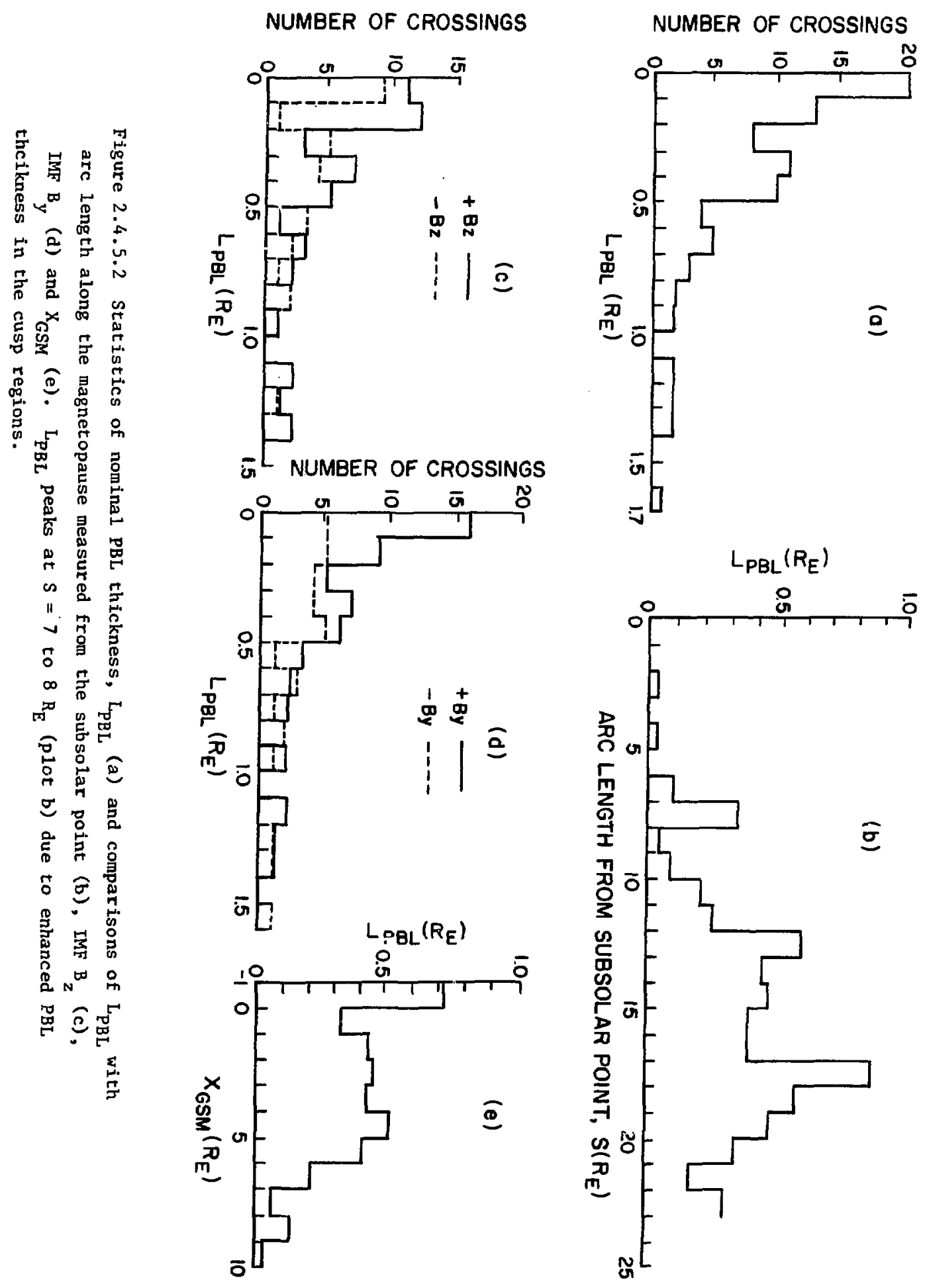
and are highly variable jue to enhanced motion of the tailward boundary relative to motions of the sunward magnetospheric boundary.

A spatial composite of nominal PBL thickness values based on IMP 6 data is given in Figure 2.4.5.3. Three thickness ranges are designated and the symbol type distinguishes two types of PBL descriptions:

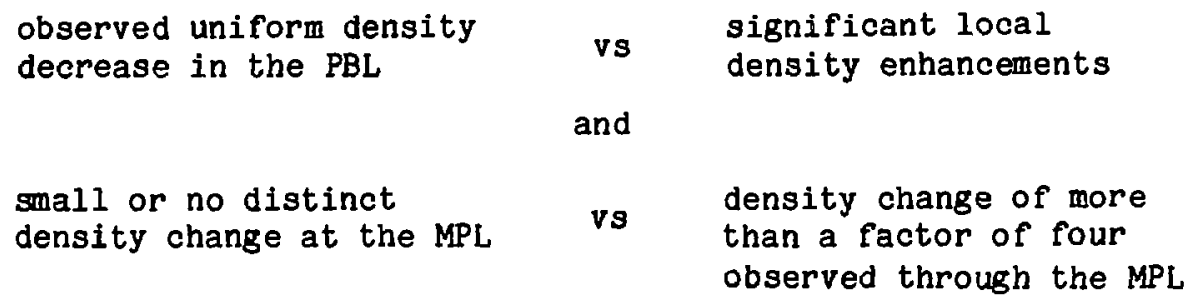

Diffusive processes are expected to produce a boundary layer that increases in thickness with increasing distance downstream; however, this prediction is not unique because a reconnection process also would lead to a steadily increasing thickness (Sonnerup, 1977). The two most significant results obtained by single satellite observations of nominal PBL thickness are (1) the high space and/or time variations observed near the magnetospheric boundary, and (2) a PBL that is typically much thicker than the MPL. 


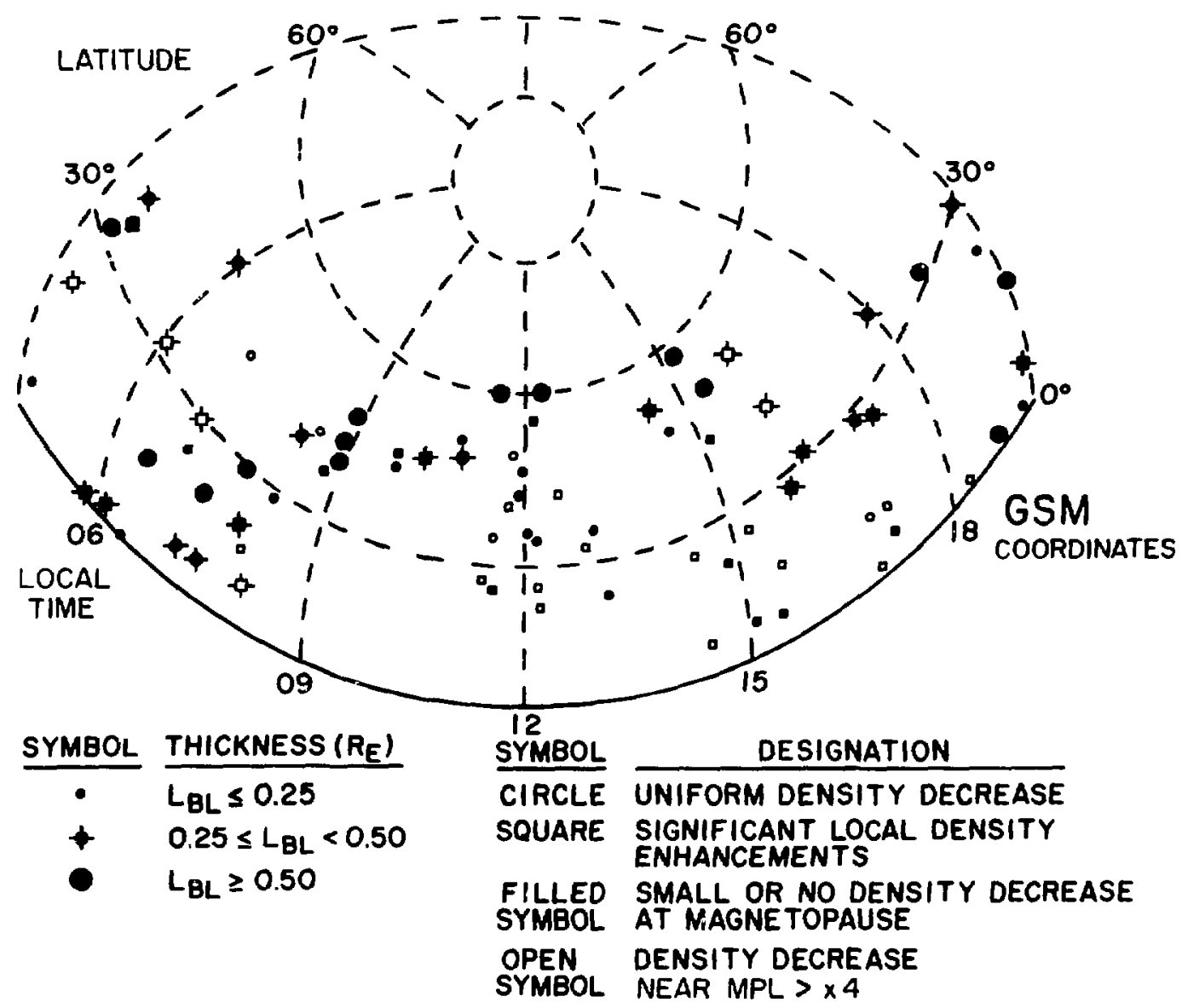

Figure 2.4.5.3 Nominal PBL thicknesses plotted on the sunward magnetopause surface. All results are based on IMP 6 crossings except for four points near local noon below $\lambda_{G S M}=30^{\circ}$ which are based on ISEE data. 


\subsubsection{Energetic Electrons Characteristics}

For several crossings, the energetic electron pitch angle Cistributions display a pancake-shaped or loss cone distribution in the PBL and in the adjacent magnetosphere. Such a pitoh-angle distribution often indicates a quasi-trapping or closed field line configuration. Minimum B pockets in the outer magnetosphere (Roederer, 1970) can lead to pancake-shaped pitch-angle distributions in the dayside region of quasi-trapping. The adjacent magnetosheath contains energetic electrons that are streaming along the magnetic field and away from the highlatitude, subsolar region of the magnetosheath. These pitch-angle distributions were obtained by sorting the 16-angle energetic electron data with the azimuthal angle of the local magnetic field based on 1.25-s averages of the magnetometer data. From 15 cases, five cases (all at local times earlier than 1300) show pancake-shaped distributions in the 1ow-latitude portion of the PBL (or LLBL). The remaining crossings had LLBL pitch-angle distributions that were more similar to the adjacent magnetospheric distributions than to the adjacent magnetosheath distributions or else the counting statistics were inadequate to make a comparison.

The usual continuity in energetic electron distributions observed in passing into the PBL from the magnetospheric side, combined with the magnetospheric-like orientation of the PBL field, provides stronger support for the presence of closed field lines in the PBL than does the occasional presence of a pancake-shaped pitch-angle distribution. This presence of closed field lines in the PBL is supported by ISEE measurements of three-dimensional distributions for $24-44.5 \mathrm{keV}$ ions. Williams 
(1978) found that trapped type distributions could be maintained "within a fraction ( $s 0.3 R_{i}$ ) of a gyroradius from the magnetosheath field where no trapping is observed."

For the five IMP 6 crossings that showed statistically significant streaming of energetic electrons in the magnetosheath, the streaming direction was consistent with a source in the subsolar region. The energetic electrons observed from IMP 6 were either streaming tailward along the magnetic field away from the subsolar region (three of the five cases) or were streaming along the magnetic field away from the cusp region (two of the five cases).

Two of the crossings showed a pancake-shaped pitch-angle distribution of energetic electrons in the magnetosheath. Large-scale inhomogeneities in the magnetosheath could temporarily produce such a distribution by setting up a mirror geometry for the field between plasma clouds that convect past the spacecraft (Fairfield, 1976). In general, the IMP 6 energetic electron data indicate that the low-latitude part of the PBL is in a closed field line region (as suggested by the observed field directions), whereas the HLBL is probably in an open field line region (Palmer and Hones, 1978). 


\subsection{Laboratory Experiments}

Laboratory experiments that provide an adequate simulation of the magnetosphere are difficult to perform. A collisionless plasma state combined with an ion gyroradius that is small compared to the scale size of the artificial magnetosphere is especially difficult to simulate. Scientists at the Space Research Institute of the USSR Academy of Sciences in Moscow have reported on a series of significant experiments using a terrella embedded in a plasma flow stream with a high Alfvén Mach number. In particular, Dubinin et al. (1976) have reported on the results of direct electric field probe measurements and the corresponding $\vec{E} \times \vec{B}$ convection pattern (Figure 2.5). A region of antisunward plasma flow is present near the magnetospheric boundary, while plasma convection with a sunward component is observed farther inward toward the terrella. Because the ion gyroradius in this experiment was on the order of a few cm, an extrapolation to the earth's magnetosphere should have a relatively thinner region of antisunward plasma flow, as observed for the PBL. Direct plasma flow measurements were also taken as an independent check of the convection results shown in Figure 2.5. Dubinin et al. (1976) conclude that measurements of the electric and magnetic field distribution show that a boundary layer with antisolar convection exists within the outer portion of the closed field line region of the laboratory magnetospheric model.

For a southward magnetic field in the plasma incident on the laboratory simulated magnetosphere, Podgorny et al. (1978a,b) report that a "visor" forms in the magnetosheath adjacent to the sunward magnetopause due to magnetic field reconnection in the cusp regions. In 


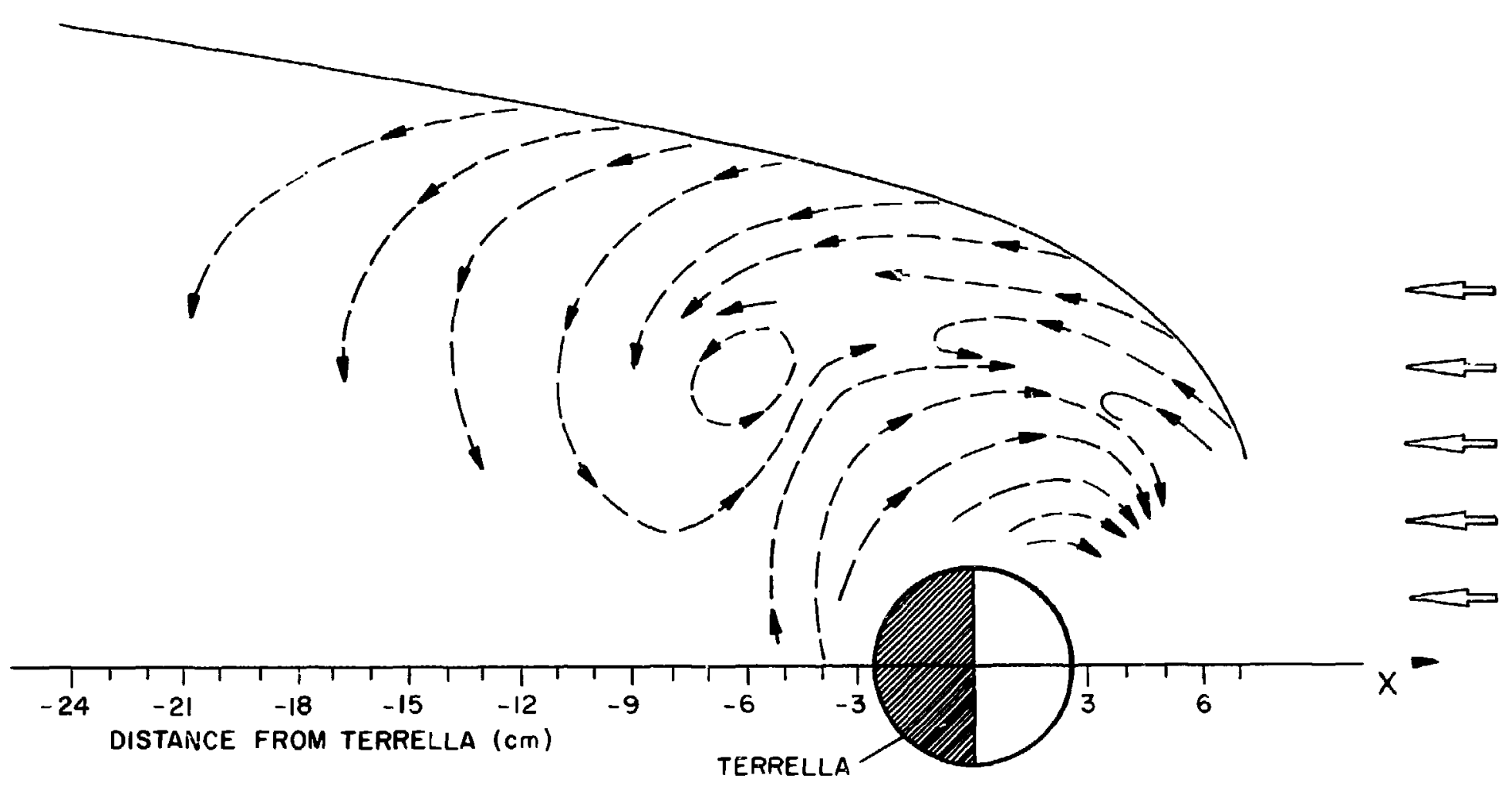

Figure 2.5 Convection pattern in the magnetospheric equatorial plane based on laboratory experiments (Dubinin et al., 1976). 
this picture, the field reversal boundary is embedded in a closed field line region external to the magnetosphere. This "visor" region is described as a region of closed field loops adjacent to the magnetopause and equatorward of the cusp regions. The PBL crossing of 4 March 1972 (see section 2.3.7) shows plasma and magnetic field signatures which indicate that the IMP 6 satellite is crossing slightly duskward of a dawnward displaced cusp region. In contrast to the visor picture, this crossing shows a clear field reversal adjacent to a PBL that has a pancake-shaped energetic electron distribution, which suggests the presence of closed field lines. The convective flow pattern predicted for the visor region includes plasma flow in the sunward direction that is not consistent with the observed flow pattern shown in Figure 2.4.3. B.U.Ǒ. Sonnerup (private communications, 1978) pointed out that, because the visor had a thickness on the order of one ion gyroradius in the laboratory experiment, the visor should be relatively thin in the real magnetosphere (where $1 \mathrm{R}_{i}=140 \mathrm{~km}$ ) due to the scaling. Sunward-component flow in this thin viscr could escape detection, however, because of the $\pm 45^{\circ}$ analyzer fan and the few available noon meridian IMP 6 crossings. These observations suggest that there is no evidence for a visor region within the limited measurement capability of the IMP 6 satellite. 


\subsection{Review of Observations}

IMP 6 plasma, energetic electron, and magnetic field observations provide several results about the structure of the MPL and low-latitude portion of the PBL (or LLBL) in the region covered by IMP 6 (see Figures

\section{2 .2 and 2.2.3)}

1. In all IMP 6 crossings, some magnetosheath-like plasma is observed earthward of the magnetopause layer (section 2.4.2). The spectral intensities of PBL electrons close to the MPL are often virtually indistinguishable from those of the adjacent magnetosheath electrons (sections 2.3.1-.8 and 2.4.2). Ion spectral intensities in the PBL and local magnetosheath are also very similar (section $2.3 .1-.8$ ). Many crossings show a significant magnetospheric contribution to the ion and electron spectra, especially farther earthward from the MPL and near relative density minima within the PBL (section 2.3 .3 ).

2. High temporal resolution (3-s average) data reveal that in 24 out of 40 IMP 6 magnetopause crossings, no distinct changes in density or electron spectra are observed at the MPL (section 2.4.2).

3. The PBL thickness is highly variable and, in general, is much greater than the MPL thickness (section 2.4.4). PBL thickness tends to increase with increasing distance from the subsolar region (section 2.4 .5$)$

4. Nominal LLBL thickness values based on 90 IMP 6 boundary crossings show no statistically significant correlation with latitude (excepting the cusp region where the PBL tends to be thicker), $K_{p}$, or with the locally measured z-component of the magnetosheath magnetic 
field. Space and/or time variations of the LLBL thicknesses may conceal any real correlation (section 2.4 .5 ).

5. Bulk plasma flow observed in the PBL by IMP 6 almost always has an antisunward component, even in the near-noon region equatorward of the cusp, and often has a significant cross-field component (section 2.4.3). IMP 6 measurements indicate that the PBL is supplied by direct transport of magnetosheath plasma across the MPL (see 2.3 sections) and that this transport is relatively widespread over the entire sunward magnetospheric boundary.

6. Energetic electron (47- to 350-keV) pitch-angle distributions suggest that the LLBL is on closed field lines (section 2.4.6). This conclusion is supported by the magnetospheric field directions observed in the PBL.

Implications of these observations will be evaluated and compared with predictions of several theoretical descriptions of the solar windmagnetospheric interaction. This will limit the possible primary sources of PBL plasma. IMP 6 observations suggest that magnetosheath plasma is the primary source of PBL plasma; however, available observations must be supplemented by a comparison with possible entry mechanisms to assess where and how the PBL is supplied. 
CHAPTER 3

THEORY AND OBSERVATIONS

\section{1 Physical Characteristics of the MPL and PBL}

\subsubsection{Plasma Characteristics}

Before making a summary of comparisons between MPL-PBL observations and theoretical models we will now review the physical characteristics of these boundary regions. Ranges in values for observed MPL and PBL plasma parameters are presented in Table 3.1.1. These values show that the plasma in these regions is a medium- $\beta$ collisionless plasma with $T_{1} / T_{e} \gg$ 1. The length scales for spatial variations of these parameters is difficult to assess based on the single-satellite measurements of IMP 6 . Estimates of length scales are given near the bottom of Table 3.1.1.

In many space science applications the simplifled form of Ohm's law is ass!med to hold so that

$$
\vec{E}+\frac{1}{c} \vec{V} \times \vec{b} \simeq \vec{J} / \sigma
$$

The "frozen-in field" approximation results in the limit of infintte conductivity $(\sigma+\infty)$ so that $\vec{E}=-\frac{1}{c} \times \vec{B}$ where $V$ is the plasma bulk velocity. This is often a good approximation because the electrical conductivity along magnetic field lines is usually very high in spac plasmas. Terms of the generalized Ohm's law neglected in the MHD approximation (see Krali and Trivelpiece, 1973, Chapter 3) include 
Table 3.1.1. Observed MPL and PBL Plasma Parameters

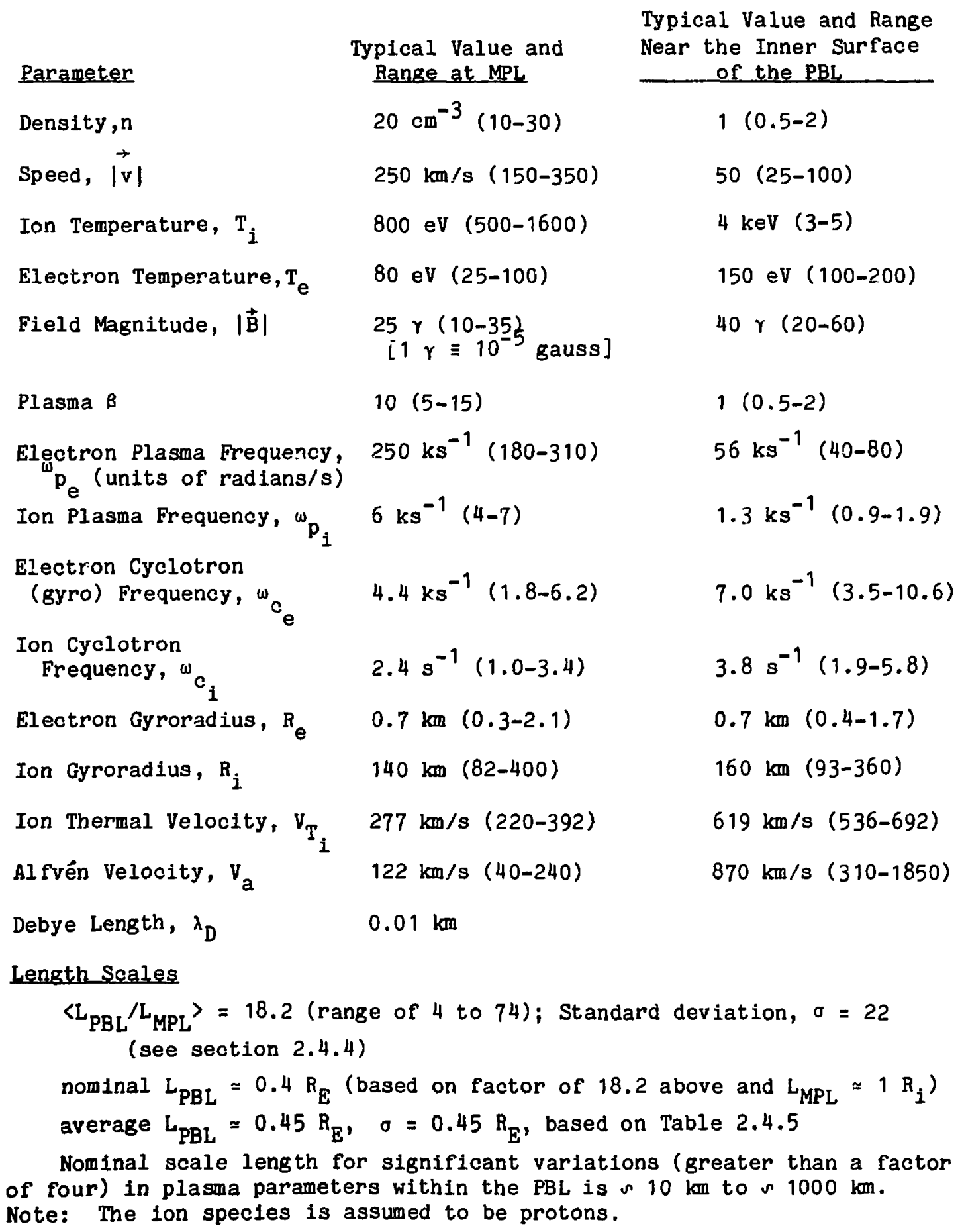




$$
\frac{\vec{J} \times \vec{B}}{n e c}, \frac{m_{e}}{n e^{2}}, \frac{\partial \vec{J}}{\partial t}, \text { and } \nabla P_{e}
$$

Their significance can be evaluated in terms of the length scale $L$ for spatial variations of the plasma parameters and the characteristic velocity $V$ of the plasma. Based on a dimensional analysis of the generalized Ohm's law and the parameter ranges observed in the MPL and PBL (see Table 3.1.1) we can evaluate the conditions under which these terms can be neglected.

$$
\frac{J \times \vec{B}}{n e c} \text { can be neglected if } \frac{L_{p_{e}}^{2}}{\omega_{c}} \frac{v_{0}}{c^{2}} \gg 1 \text {. }
$$

From Table 3.1.1,

$$
0.05<\frac{{ }_{p_{e}}^{2}}{{ }_{{ }_{c}}^{2}} \frac{v_{0}}{c^{2}}<8 \text {, using typical values across the PBL. }
$$

Thus, $\vec{J} \times \vec{B} /(n e c)$ cannot, in general, be neglected.

$$
\frac{m_{e}}{n_{e}^{2}} \frac{\partial J}{\partial t} \text { can be neglected if } \frac{L^{2} \omega_{p}^{2}}{c^{2}} \gg 1
$$


From Table 3.1.1,

$2<\frac{L^{2} \omega_{p}^{2}}{c^{2}}<1 \times 10^{6}$, using the full range of values across the

PBL.

Thus, $\frac{m_{e}}{n_{e}{ }^{2}} \frac{\partial J}{\partial t}$ can be neglected except for fluctuations involving

length scales less than $\mathrm{s} 10 \mathrm{~km}$ near the inner surface of the PBL.

$\nabla P_{e}$ can be neglected iff $\frac{i . \omega_{c} c_{e}}{\mathrm{kT}_{\mathrm{e}} \mathrm{m}_{\mathrm{e}}} \gg 1$

From Table 3.1.1,

$0.025<\frac{L V \omega_{c}}{k \mathrm{~T}_{e} / \mathrm{m}}<500$, with a typical value of 14 , using the full range of values across the PBL.

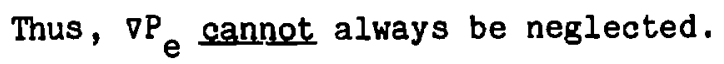

Finite Larmor radius effects can be neglected if $\frac{{ }^{L \omega} c_{i}}{\sqrt{\mathrm{KT}_{i} / \mathrm{m}_{i}}} \gg 1$.

From Table 3.1.1,

$0.04<\frac{L \omega_{c}}{\sqrt{\mathrm{kT}_{1} / \mathrm{m}_{i}}}<11$, with a typical value of 1.3 , using the full range of parameters observed in the PBL. Thus, finite Larmor radius corrections cannot, in general, be neglected. 
These results suggest that the frozen-in-field approxiamtion as well as the simplified form of ohm's law are not reliable for scale lengths and plasma parameter ranges that are often observed in the MPL-PBL region.

\subsubsection{MHD Discontinuities}

The MPL represents a discontinuity in a magnetized plasma. An analysis of such discontinuities is based on magnetohydrodynamic (MHD) conservation equations (e.g., Landau and Lifshitz, 1960, Chapter VIII, or Nishida, 1978). For the MHD discontinuity analysis, a frame of reference is chosen in which the discontinuity is stationary. Several assumptions are then made about the plasma and field characteristics, including

(1) the ion gyroradius is small compared to all other scale lengths (Lynn, 1967), and

(2) the pressure is sufficiently isotropic that it can be expressed as a scalar."

The first assumption results from the MHD ordering of scale lengths (see Krall and Trivelpiece, 1973, section 3.7). IMP 6 observations indicate that the MPL thickness is typically on the order of one ion gyroradius. Consequently, MHD discontinuity analyses have limited applicability to the MPL.

Hudson (1971) and Iranov (1974) have evaluated the effect of anisotropic pressures on a discontinuity analysis. The general effect is to complicate the identification of a particular discontinuity. These are also one fluid treatments and are limited by the assumption of stationarity. 
Brackets [] are used to denote differences of a given quantity from the downstream to the upstream state. Thus, $[P]=$ (downstream pressure - upstream pressure). Components along the normal to the boundary are given the subscript $n$. Using this notation, conservation of mass can be expressed as $\left[\rho v_{n}\right]=0$ and conservation of magritic flux can be writter as $\left[B_{n}\right]=0$.

Two types of MHD discontinuities result depending on whether $v_{n}$ is zero or nonzero. For $v_{n}=0$ (a tangential or contact discontinuity), there is no mass flow across the boundary and the discontinuity is convected along with the flow. If, in addition, $B_{n}=0$, then it is a tangential discontinuity for which the total plasma and field pressure across the discontinuity is conserved $\left(1 . e .,\left[P+\left(B^{2} / 8 \pi\right)\right]=0\right)$. In a contact discontinuity, $B_{n} \neq 0$ and plasma would tend to rapidly smooth the discontinuity by diffusion along field lines.

For $v_{n} \neq 0$ plasma flows across the discontinuity which then propagates through the medium. The resulting waves are large amplitude, sharp structures, or shock waves. From the momentum equation and conservation of mass, three solutions obtained are (1) intermediate shocks (Alfyén shock or rotational discontinuity), (2) fast shocks, and (3) slow shocks.

Jump conditions for these shock waves and discontinuities are given in Table 3.1.2 (from Nishida, 1978, p. 17). An asterisk denotes an arbitrary but nonzero change and a blank marks an unspecified change. 
Table 3.1.2. Classification of Shock Waves and Discontinuities

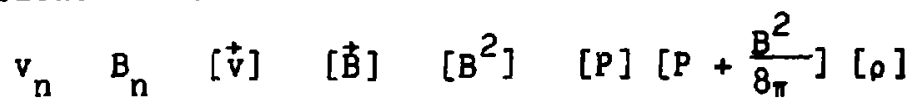

\begin{tabular}{|c|c|c|c|c|c|c|c|c|}
\hline Tangential discontinuity & 0 & 0 & & & & & 0 & \\
\hline Contact discontinuity & 0 & $\star$ & 0 & 0 & 0 & 0 & 0 & * \\
\hline Intermediate shock & 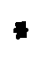 & 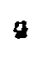 & 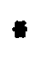 & $*$ & 0 & 0 & c & 0 \\
\hline Fast shock & - & & 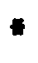 & $*$ & + & + & + & + \\
\hline Slow shock & 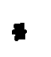 & * & $*$ & $\neq$ & - & + & + & \\
\hline
\end{tabular}

[]: jump in the given variable

In general, observed characteristics across the MPL are most compatible with a rotational or a tangential discontinuity. At the highest available time resolution, it is still difficult to clearly describe the discontinuity. This difficulty is probably due to the lack of stationarity during the sampling period.

Observed plasma and field characteristics across the MPL are often consistent with a rotational discontinuity. From a set of 57 MPL crossings with OGO 5, Sonnerup (1977) found that 19 cases ( $533 \%$ ) had a significant normal component $B_{n}$ for which $B_{n}>3 \sigma_{B}$. Similarly, two IMP 6 crossings presented in section 2.3 (14 April 1972 and 4 March 1972) had a significant normal field component and could be consistent with a rotational discontinuity. 


\subsubsection{Comparisons of Spectra}

Liouville's theorem requires that the phase space density $f$ of a plasma remains constant along dynamical trajectories, provided the colIisionless Boltzmann equation $[\mathrm{df} / \mathrm{dt}=0]$ applies.

The differential flux $J$ is related to $f$ by

$$
f=m J / v^{2}
$$

(Rossi and Olbert (1970), Chapter 1). In the case of PBL plasma, the electron differential flux spectra observed on each side of the MPL are nearly indistinguishable. We have also compared some measured velocity distributions plotted in terms of phase space density on each side of the MPL and found a similar close relationship. Both of these observations indicate that $J / \mathrm{V}^{2}$ and, hence, $f$ are often nearly equivalent on opposite sides of the MPL. These observations suggest that the dynamical trajectories for PBL plasma particles that are located close to the MPL map back into the nearby magnetosheath. However, because $f=$ $f(\vec{x}, \vec{v}, t)$ includes seven variables, arguments concerning source populations based on a comparison of phase space distributions is difficult to extend beyond tine above qualitative discussion.

If the PBL plasma source is supplied only by a remote entry point, the commonly observed continuity in plasma parameters across the IAPL would be very improbable. Available magnetosheath observations (e.g., Siscoe et al., 1968; Kaufmann and Horng, 1971) indicate that it consists of intertwined filamentary plasma structures or plasma clouds with a scale size of $1 \mathrm{R}_{E}$ or less. IMP 6 observations suggest that the scale 
size of inhomogeneities in the PBL ranges from $10 \mathrm{~km}$ to $\mathrm{s} 1000 \mathrm{~km}$ in a direction transverse to the magnetopause surface. Even for a remote entry point for magnetosheath plasma, inhomogeneities in the PBL are likely to have a length of $1 R_{E}$ or less, depending on the source mechanism. Thus, the continuity of plasma parameters across the MPL indicates that PBL plasma located close to the MPL is supplied by transport of magnetosheath plasma across the MPL at positions that are not far removed from the points of observation.

For PBL plasma observed farther earthward from the MPL, the source regions can be relatively remote. How remote the plasma source region is in a given PBL crossing is not clear from available information. However, the more closely related the PBL density and spectra are to the nearby magnetosheath values, the closer the source region is likely to be. Usually, PBL spectra observed across the MPL and through the PBL during a given crossing are more closely related than spectra observed near the MPL during different crossings (e.g., compare Figures 2.3.5.6 and 2.3.7.6). During local density minima in the PBL the spectra become more magnetospheric-like.

These observations suggest that magnetosheath plasma supplies the major portion of PBL plasma by direct transport across the MPL and that magnetospheric plasma becomes simply mixed in with this continuously replenished plasma population. Values of $\bar{E}$ (mean thermal energy per particle) often remain constant across the MPL, even with the beginning of a decrease in plasma density (e.g., see Figure 2.3.2.1). $\vec{E}$ then increases, mainly in the inner portion of the $\mathrm{PBL}$, in a manner consistent with simple mixing. Recent ISEE ion measurements (Haerendel et 
al., 1978b) indicate that the PBL comprises magnetosheath plasma (identified by the presence of energetic $\mathrm{He}^{++}$) and magnetospheric plasma (identified by the presence of $\mathrm{He}^{+}$as well as $\mathrm{O}^{+}$for one reported case). By considering simple mixing of magnetosheath and magnetospheric plasma in the PBL, it is unnecessary to hypothesize any energization process (which would be required if the sole source for the PBL is the adjacent magnetospheric plasma population). In fact, the energy density always decreases in passing from the magnetosheath, through the MPL and PBL, into the magnetosphere. The general similarity of PBL and magnetosheath spectra suggests that the reduced flow velocity normally observed in the PBL corresponds to a net dissipation of plasma energy [note that

the total plasma energy density is given by $\left.u=n\left(\frac{3}{2} k T+\frac{1}{2} m v^{2}\right)\right]$. During MPL-PBL crossings, a tailward "draping" of the outer magnetospheris field is often indicated by the crossing location and measured field directions. This effect is expected in the presence of crossfield plasma flow in the PBL because some of the momentum of the oncoming magnetosheath plasma can be taken up by stress in a tailward draping of the field while some of the momentum can be transferred to the ionosphere by field-aligned currents (Eastman et al., 1976). 


\subsubsection{Particle and Energy Flux in the PBL}

The PBL thickness is highly variable and has a characteristic thickness that is generally much greater than that of the MPL (current layer). PBL plasma with typical values of density, velocity, and energy flowing through a partial annulus of thickness $\sim 1 / 3 R_{E}$ located at the dawn-dusk meridian plane will be considered including a factor of 0.3 to restrict the annulus to the low-latitude portion of the PBL - see Figure 1.1. Table 3.1 .4 gives ion plasma parameters for a typical PBL crosssection at the dawn-dusk meridian plane. This cross-section is separated into four radially symmetric zones to illustrate typical variam tions of parameters within the PBL.

Table 3.1.4. Ion Plasma Parameters for a Typical Dawn-Dusk Meridian Cross-Section of the PBL

\begin{tabular}{|c|c|c|c|c|c|c|}
\hline $\begin{array}{c}\text { Plasma } \\
\text { Parameter }\end{array}$ & $\begin{array}{l}\text { Magneto- } \\
\text { sheath }\end{array}$ & $\begin{array}{c}\text { PBL } \\
1\end{array}$ & $\begin{array}{c}\text { PBL } \\
2\end{array}$ & $\begin{array}{c}\text { PBL } \\
3\end{array}$ & $\begin{array}{c}\text { PBL } \\
4\end{array}$ & $\begin{array}{l}\text { Magneto- } \\
\text { sphere }\end{array}$ \\
\hline$n\left(\mathrm{~cm}^{-3}\right)$ & 18 & 14 & 10 & 6 & 2 & 0.5 \\
\hline$|\overrightarrow{\mathrm{v}}|(\mathrm{km} / \mathrm{s})$ & 300 & 280 & 200 & 120 & 50 & $<30$ \\
\hline $\bar{E}(\mathrm{keV})$ & 0.5 & 1 & 2.5 & 4 & 6.5 & 7 \\
\hline $\begin{array}{l}\mathrm{n}|\overrightarrow{\mathrm{V}}|\left(\mathrm{cm}^{-2} \mathrm{~s}^{-1}\right) \\
\quad\left(\begin{array}{ll}\left.\mathrm{x} 10^{8}\right)\end{array}\right.\end{array}$ & 5.4 & 3.9 & 2.0 & 0.72 & 0.10 & $\sim 0$ \\
\hline $\begin{array}{c}\mathrm{n}|\overrightarrow{\mathrm{V}}| \overline{\mathrm{E}}\left(\frac{\mathrm{keV}}{\mathrm{cm}^{2} \mathrm{~s}}\right) \\
\left(\because 10^{8}\right)\end{array}$ & 2.7 & 3.9 & 5.0 & 2.9 & 0.7 & $\sim 0$ \\
\hline
\end{tabular}


For an annulus of $16 \mathrm{R}_{\mathrm{E}}$ radius, the cross-sectional area of our truncated annulus is $4.1 \times 10^{18} \mathrm{~cm}^{2}$. Based on the values in Table 3.1 .4 and averaging the four PBL subdivisions, the total particle flux flowing tailward into the flanks of the magnetotail is $\sim 6.9 \times 10^{26} \mathrm{~s}^{-1}$, and the total energy flux is $\sim 2 \times 10^{11} \mathrm{~W}$, which is more than adequate to compensate the estimated quiet time loss of plasma from the plasma sheet (Hill, 1974) and the energy dissipation in the quiet time aurora. If we assume that tailward flowing PBL plasma comprises s20\% mixed-in magnetospheric plasma and magnetosheath plasma that was transported across the sunward MPL at positions no more than a few $R_{E}$ upstream from the points of observation, we can characterize the PBL source by the locally measured magnetosheath values given in Table 3.1.4. For this situation, the $6.9 \times 10^{26}$ particles/s flowing into the magnetotail had $\backsim 6 \times 10^{11} \mathrm{~W}$ more power before entering the LLBL. By comparison, a few times $10^{10} \mathrm{~W}$ is considered adequate to drive the steady-state magnetospheric convection circuit (Hill and Wolf, 1978). This estimate depends on the sources ascrihed to the PBL plasma. 


\subsection{Observations and Theory}

3.2.1 Early Models of the Solar Wind-Magnetosphere Interaction

This section provides a comparison of IMP 6 observations of the MPL and PBL with models of the solar wind-magnetosphere interaction. Some early models of this interaction are

(1) two-fluid (cold plasma) models (e.g., Ferraro, 1952),

(2) viscous fluid models (e.g., Axford and Hines, 1961), and

(3) large-scale, steady-state reconnection (e.g., Dungey, 1961). A brief description of these early models along with specific predictions is itemized and compared with our observations.

Two-Fluid (Cold Plasma) Models. The first basic model of the magnetopause layer was developed by Ferraro (1952). It (Figure 3.2.1.1a) considers the "boundary layer" between an unmagnetized, collisionless cold plasma stream and a vacuum geomagnetic field. As magnetosheath Ions and electrons enter the MPL they are deflected in opposite directions by the Lorentz force, $\frac{g}{c} \vec{v} \times \vec{B}$. The plasma particles return to the magnetosheath with an equal and oppositely directed velocity (i.e., specular reflection). A current (the "Chapman-Ferraro" current) is generated by transverse motion of plasma in the MPL. Because of their larger mass-to-charge ratio, the ions penetrate farther into the geomagnetic field than the electrons. The resultant polarization eleccric field opposes the charge separation and leads to a current layer that has a thickness comparable to the plasma skin depth $(\approx 1 \mathrm{~km})$. Whereas the ions are mainly deflected by the polarization electric field, the alectrons are accelerated by this field as they are deflected by the magnetic field. Thus, the Chapman-Ferraro current is carried 


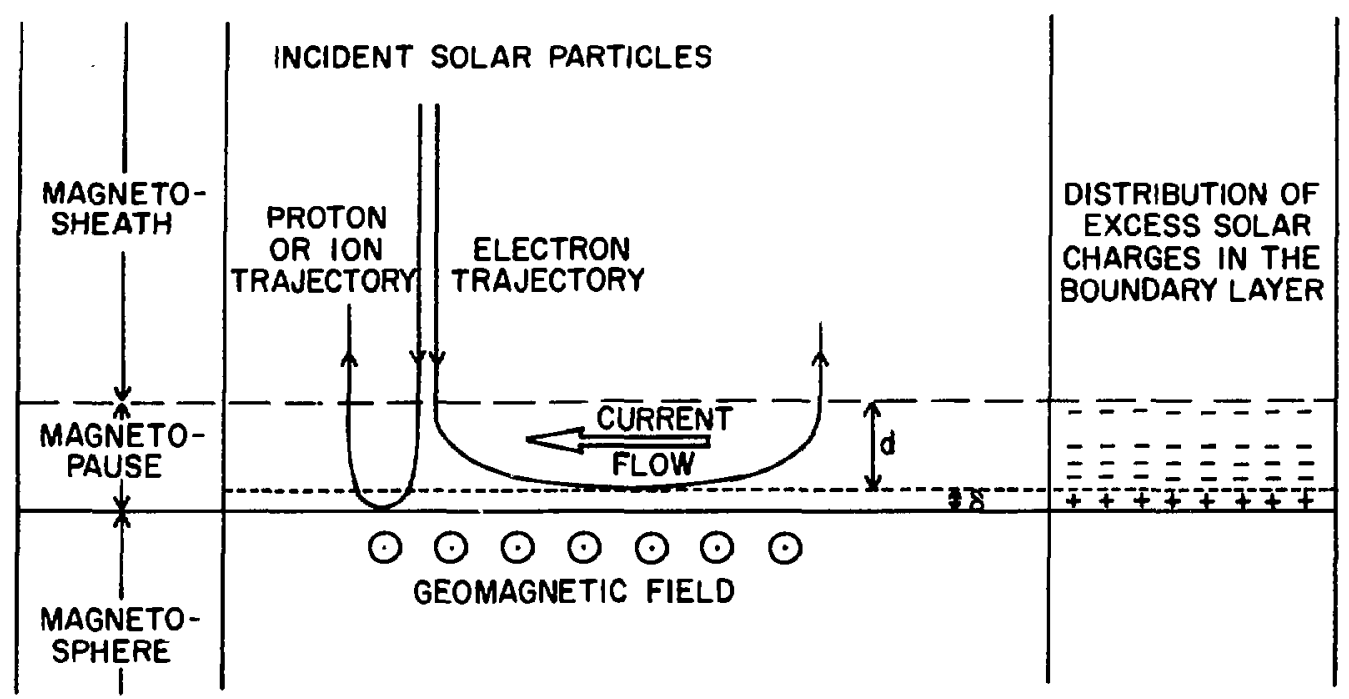

(a) FERRARO MAGNETOPAUSE MODEL

$$
(d \sim \mid k m, \delta \sim 1 \mathrm{~m})
$$

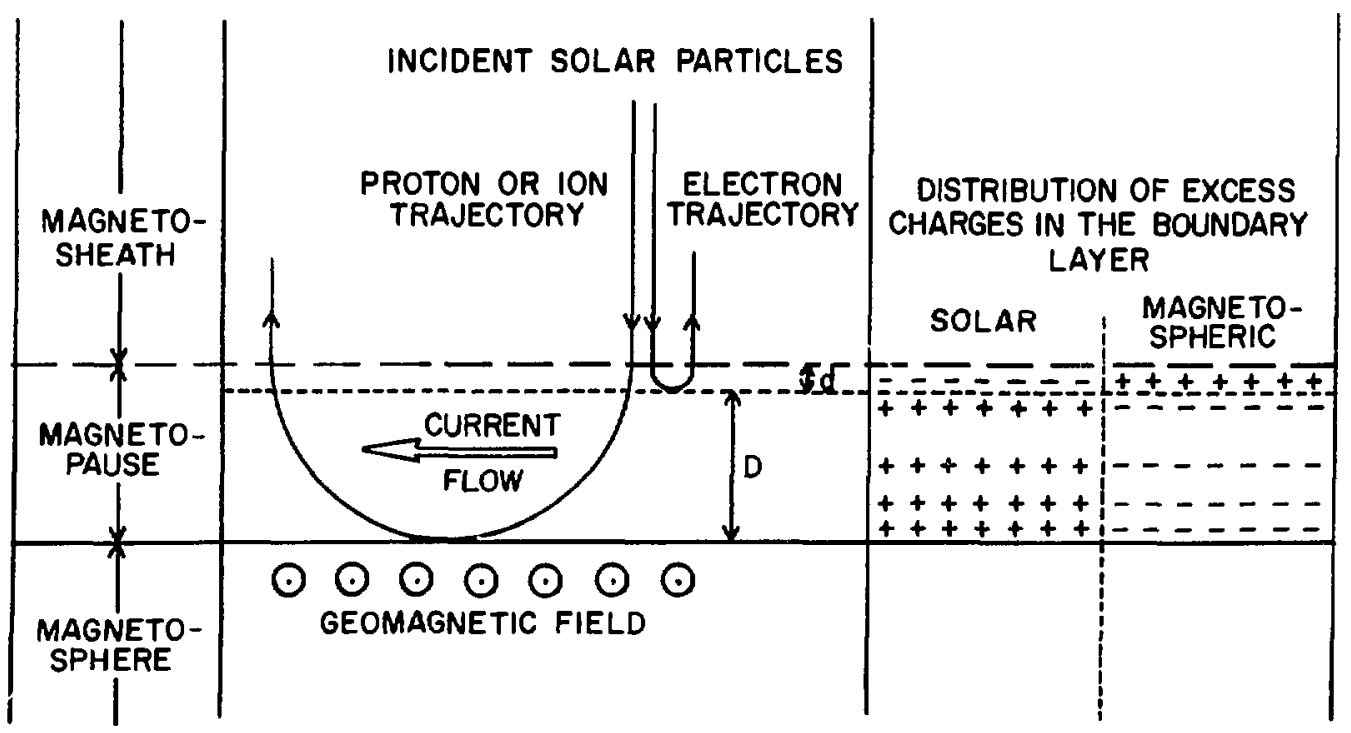

(b) PARKER MAGNETOPAUSE MODEL

$$
(D \sim 100 \mathrm{~km}, \mathrm{~d} \sim 1 \mathrm{~km})
$$

Figure 3.2.1.1 Plasma characteristics in the Ferraro (a) and Parker (b) magnetopause models (Willis, 1971). 
primarily by the electrons and energy is iransferred from ions to the electrons. These results were obtained for an idealized plane MPL in which magnetosheath plasma is incident normal to the MPL. The "Ferraro magnetopause" is useful in visualizing the generation of the current layer.

Parker (1967) considered the magnetopause structure in the limit of complete neutralization of the polarization field by ambient magnetospheric plasma. The "Parker magnetopause" model is shown in Figure 3.2.1.1b for a plane boundary and normal incidence of magnetosheath plasma. No energy is transferred from ions to electrons in the steady state and the ions carry the main part of the current due to their much larger cyclotron radii. The Parker magnetopause thickness is i $1 R_{i}$, which is fairly consistent with observations.

Both models neglect the magnetosheath field and the magnetospheric plasma. The continuity in field magnitude and plasma parameters that is commonly observed across the MPL is inconsistent with these models; neither can explain the presence of the PBL because they do not provide for plasma transfer across the MPL.

Viscous Fluid Models. Axford and Hines (1961) introduced a viscous fluid interaction model as part of a general description of magnetospheric convection as illustrated in Figure 3.2.1.3. This model involves momentum transfer, without plasma transfer, across the magnetospheric boundary. Freeman, et al. (1968) applied this model in explaining their observations of the PBL with the Rice University suprathermal 
Ion detector on the ATS 1 satellite. The existence of the PBL is consistent with a viscous interaction mechanism; however, the unique similarity of plasma density and spectra across the MPL can only be considered as fortuitous in this model since only momentum transfer (and not plasma transfer) is considered. However, magnetospheric plasma which replaces the magnetospheric plasma that gets mixed with magnetosheath plasma in the PBL could be involved in a general convection pattern as shown in Figure 3.2.1.3.

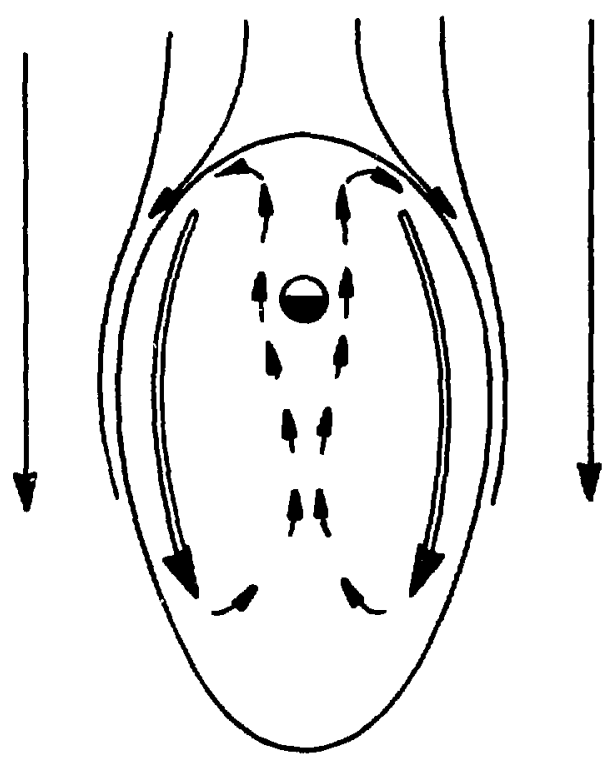

Figure 3.2.1.3 Magnetospheric convection pattern produced by a viscous interaction near the magnetopause (Axford and Hines, 1961). 
Large-scale, steady-state reconnection The reconnection model (Dungey, 1961; Levy et al., 1964; also see the review by Vasyliunas, 1975), as piatured in Figure 3.2.1.4, predicts a correlation between the z-component of the magnetosheath field and geomagnetic activity, and also prediats rapid access of solar flare particles into the polar cap. After observations were made supporting these predictions the reconnestion model became firmly established. Johnson (1978) noted that, between 1968 and 1975, the reconnection model was "virtually unchallenged." As described by Vasyliunas (1975), there are many reconnection theories based on either a single-particle or a hydromagnetic approach. We will discuss only the most commonly stated predictions of the hydromagnetic approach .

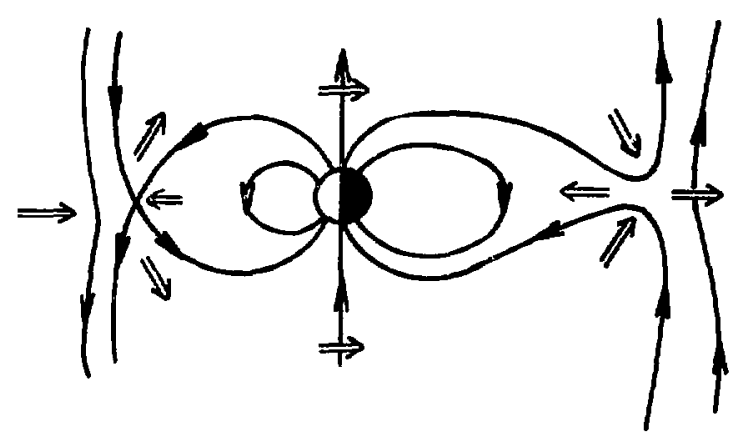

Figure 3.2.1.4 Plas na flow and field configuration in the Dungey reconnection model (Dungey, 1961). 
Magnetic reconnection involves plasma flow across a separatrix, a surface that separates topologically distinct magnetic field line regions, accompanied by a transport of magnetic flux through a small diffusion region in which a magnetic neutral poirst is embedded. Associated with the reconnection process is an electric field directed at right angles to $B$ and located along the separatrix surface. This reconnection electric field necessarily accelerates the plasma and most reconnection models predict a high-speed jet of plasma directed away from the diffusion region and traveling near the magnetospheric boundary .

Figure 3.2.1.5 shows predicted plasma and field parameter changes near the magnetopause based on the model of Yang and Sonnerup (1977). In this example the plasma jet velocity is $500 \mathrm{~km} / \mathrm{s}$ (above the nearby magnetosheath flow velocity) just inside the magnetopause and increases to $900 \mathrm{~km} / \mathrm{s}$ at the inner surface of the expansion fan. The PBL in this model ilocated between the magnetopause and the inner surface of the slow expansion fan) has a total angular width of $2^{\circ}$. Relative values of plasma parameters for this IMP 6 crossing are plotted as dashed lines in Figure 3.2.1.5. Here, the intermediate wave (IW) is tht MPL location and the beginning of the slow expansion fan (SEF) is taken as the inner surface of the PBL. Predicted temperature and velocity profiles are nearly opposite to the observed variations.

The high-speed outflow from the subsolar region predicted by most versions of the reconnection picture (Heikkila, 1978) has not been clearly observed in any of more than 225 IMP 6 crossings at a time resolution of $\simeq 100$ seconds. At a time resolution of $\simeq 12.5 \mathrm{~s}$, none of 

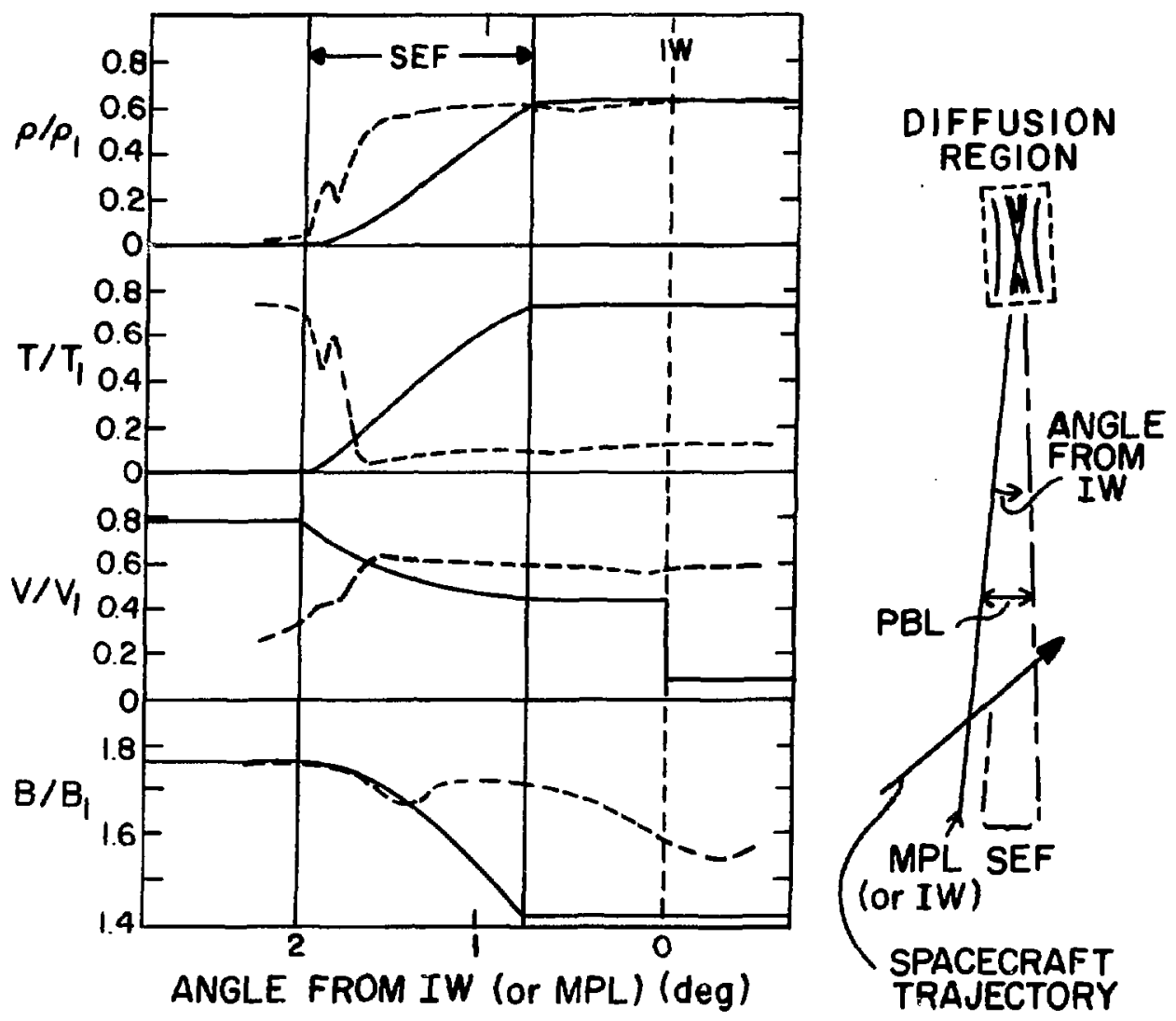

Figure 3.2.1.5 Calculated plasma and field parameters (solid lines) based on a reconnection model (Yang and Sonnerup, 1977) compared with observed parameters (dashed lines) for the 14 April 1972 IMP 6 crossing of the MPL-PBL region. Angles subtended relative to the subsolar point are plotted along the abscissa. The PBL thickness has been plotted to fit between the intermediate wave (IW) or magnetopause and the earthward edge of the slow expansion fan (SEF). The geometry for this comparison is illustrated at the right side of this figure. 
40 dayside MPL-PBL crossings selected for high time resolution analysis exhibited an obvious accelerated flow. Velocity enhancements (generally s $50 \%$ in magnitude) are observed in less than $10 \%$ of the IMP 6 crossings. Many of these cases can be explained in terms of directional shifts of the Ion velocity distribution upon entering the $\mathrm{PBL}$.

3.2.2 Recent Models of the Solar Wind-Magnetosphere Interaction Difficulties encountered in the early interaction models and the significance of the interaction for magnetospheric processes in general, have inspired a resurgence of interest in developing improved models. Most of the recent interaction models emphasize the three-dimensional, non-steady-state character of the MPL and PBL. "Mechanisms that provide for space and time variations of magnetosheath plasma penetration into the PBL could explain many IMP 6 observations, including the observation that the PBL thickness is highly variable $=n d$, in general, is much larger than the MPL thickness. However, although the PBL plasma flow has signiflcant fluctuations compared to the nearby magnetosheath plasma flow, the flow field is still almost always antisunward and is generally well ordered.

Some recent models of the solar wind-magnetosphere interaction are

(1) sporadic reconnection (Sonnerup, 1978; Schindler, 1978)

(2) convective turbulence (e.g., Haerendel, 1978)

(3) particle orbit theory (e.g., Cole, 1974)

(4) impulsive penetration (e.g., Lemaire and Roth, 1977)

(5) diffusion by plasma kinetic instabilities (e.g., Eviatar and Wolf, 1968; section 3.3), and

(6) bimodal interaction model (Crooker, 1977). 
Brief descriptions of these models with specific predictions are itemized and compared with our observations.

Speradic reconnection Because the large scale laminar reconnection flow pattern has not been observed, Sonnerup (1978) and Schindler (1979) have recently proposed a "patchy" forced reconnection picture to avoid this difficulty. In this mechanism, magnetosheath irregularities near the magnetopause are considered to induce time-limited, localized merging regions. Because these merging regions are highly time variable they would not lead to a large-scale laminar reconnection pattern. Schindler (1979) points out that sporadic merging patches would, in general, not allow for an ordered flow field inside the magnetopause. The observed PBL flow, however, is generally well ordered apart from the cusp regions, although it has enhanced fluctuations in plasma bulk flow compared to the nearby magnetosheath.

Considering the lack of notable accelerated flow, perhaps the dissipation involved in the reconnection process leads to enhanced thermal energy instead of increased kinetic energy. In this case the mean thermal energy per particle should increase near the MPL resulting in a profile similar to that shown in Figure 3.2.2.1a. However, the observed profile of mean energy per particle is usually similar to the profile shown in Figure 3.2.2.1b. 


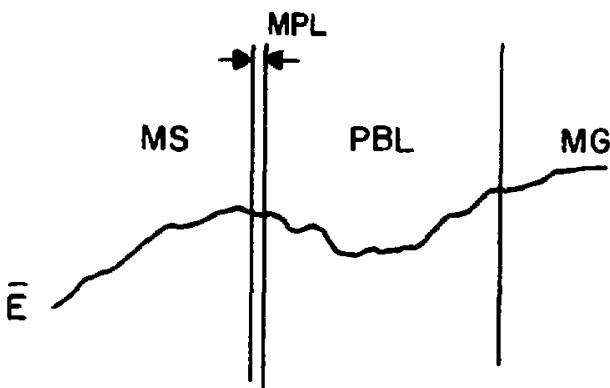

(a)

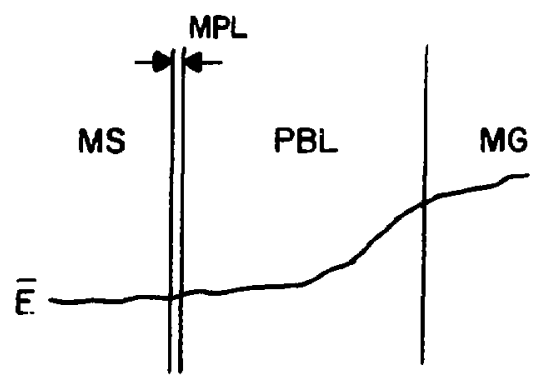

(b)

Eigure 3.2.2.1 Comparison of expected (a) and observed (b) profiles of mean energy per partiole.

In contrast to the prediction of reconnection theories that tnere should be net plasma energization at the expense of eleatromagnetis field energy, IMP 6 observations suggest that there is net de-encrgization of PBL plasma (see sections 3.1 .3 and 3.1 .4 ). Heikkilz (1973) noted that available observations of the PBL, inoluding the lask of net plasma energization, indicate that the PBL acts as a generator of electroinagnetis energy and not as a load.

Convective Turbulence observations from HEOS 2 and IMP 6 often show irregular flow patterns in the cusp regions (Fizure 2.4.3). These observations have led Haerendel (1979) to propose tnat eddy convection in tho cusp regions san lagd to signifizant anomalous diffusion of magnetosheath plasma. Haerendel (1973) nolis that reconnection processes are expected to be assosiated witn tnis turbulence. In these 
reconnection events, free energy in the magnetic configuration is converted into kinetic energy or turbulent wave energy. These events are expected because the local magnetosheath magnetic field will always be antiparallel to some set of field lines in the cusp indentation (see Figure 1.1). In addition, Haerendel (1978) stated that "there are various additional sources of free energy [i.e., not involving reconnection] which can feed microscopic turbulence."

The eddy convection picture is supported by the observed irregular flow pattern in the cusp regions and by physically reasonable comparisoxis with observed fluid turbulence phenomena. Haerendel et al. (1978) and Johnson (1978) both consider the cusp regions to be the basic PBL source. Johnson considers a PBL populated by magnetosheath plasma by way of the cusp regions only. Haerendel suggested, in addition, that heat conduction and wave propagation with subsequent dissipation along the magnetic field could supply the observed energy and ion mobility of the LLBL. This source mechanism could often lead to plasma flow in the noon meridian, equatorward of the cusp regions, that has a sunward flow component.

We have found only four isolated cases of plasma flow in the PBL with a sunward flow component and three of these crossings were located near the cusp regions. The exceptional case occurred on 9 May 1972 (see Appendix B). In each case the plasma flow with a sunward component was of short duration and was preceded and followed by the predominant antisunward flowing plasma. In all other IMP 6 crossings, PBL plasma with a sunward flow component has not been observed. Even though the IMP 6 plasma analyzer only samples up to $\pm 45^{\circ}$ about the ecliptic plane, five 
crossings near the noon meridian have been identified where the field direction is within the electrostatic analyzer aperture. This orientation insures that a field-aligned flow would be sampled even with unusually low temperature of the flowing plasma. In a sixth crossing (14 April 1972, located at - $23^{\circ}$ latitude - see section 2.3.5) the field angle was only $10^{\circ}$ out of the sampling cone, which still allows a reliable determination of the ecliptic plate component of the bulk flow for magnetosheath-like ion distributions (see Appendix A). These flow characteristics are summarized in Figure 2.4.3.

Flow directions observed in the PBL by IMP 6 suggest that plasma in the cusp regions is supplied in part by PBL plasma transported across the MPi somewhere equatorward of the cusp regions. In addition, the source of PBL plasma near the noon meridian, equatorward of the cusp regions, must be primarily magnetosheath plasma transported across the MPL in this same region (Eastman and Hones, 1978). Particle Orbit Theory $K$. Cole (1974) investigated the effects of nonadiabatic particle motion near the magnetopause and found that particles with angles of approach to the magnetopause that are less than a critical angle with respect to the magnetopause surface can be transported across the MPL. This theory predicts a hardening of the PBL differential flux spectrum immediately earthward of the MPL because particles with higher thermal energy and larger gyroradii also have larger critical entry angles. Although this prediction is not clearly consistent with IMP 6 observations, further studies of nonadiabatic particle motion that include the PBL are needed. 
Impulsive Penetration $\mathrm{J}$. Lemaire and coworkers have developed $a$ theory of impulsive penetration of magnetosheath plasma elements (Lemaire and Roth, 1978; Lemaire, 1977, 1979; Lemaire et al., 1978) that is depicted in Figure 3.2.2.2. Large-scale inhomogeneities in the magnetosheath are considered which would include plasma clouds with excess mass and momentum. As these plasma clouds interact with the magnetospheric boundary, polarization charges are established that can be neutralized by depolarizing currents that link the MPL-PBL with the ionosphere. Kinetic energy of the penetrating plasma cloud is dissipated in the ionosphere by Joule heating. Local dissipation effects may also be

\section{LARGE-SCALE INHOMOGENEITIES} IN THE MAGNETOSHEATH

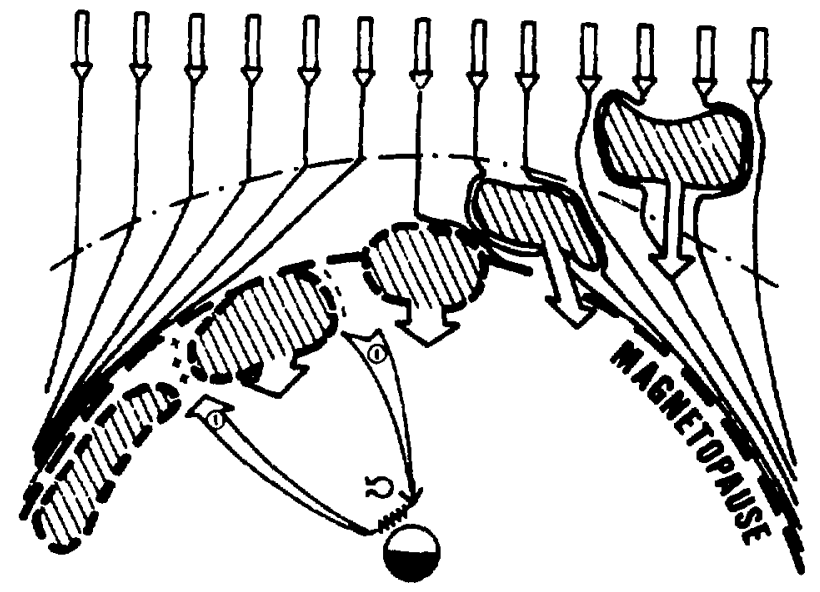

Figure 3.2.2.2 The impulsive penetration model using an equatorial cross-section of the magnetosphere (Lemaire and Roth, 1978). 
present that are associated with the penetration process. Although the details of the MPL interaction and the associated dissipation mechanisms are not fully developed in this theory, it represents an intriguing possibility based on a comparison of its prediotions with observations (Lemaire, 1979).

The impulsive penetration model considers the PBL to be a magnetohydrodynamic (MHD) generator or unipolar dynamo (Coleman, 1971). Eastman et al. (1976) have discussed the implications of IMP 6 as well as low altitude satelite observations for the MHD generator modei of the PBL. This mechanism is implied by IMP 6 observations of

(1) significant cross-field flow in the PBL (e.g., section 2.3.5),

(2) energetic electron and magnetic field characteristics that indicate the presence of closed field lines in the LLBL (sections 2.3 and 2.4 .6 ),

(3) de-energization of PBL plasma (sections 3.1 .3 and 3.1 .4 ), and

(4) tailward draping of the outer magnetospheric field. Although the impulsive penetration model emphasizes the effect of large-scale inhomogeneities in the magnetosheath plasma source, the MHD generator mechanism can operate effectively with small scale inhomogeneities such as those observed by IMP 6 as large density fluctuations in high time resolution (section 2.4 .2 ). These density gradients can provide a source of free energy to drive plasma instabilities (section 3.3). The resultant field fluctuations can scatter plasma particles and lead to significant cross-field diffusion of magnetosheath plasma into the PBL. 
Bimodal interaction model. Crooker (1977) has introduced a geometrically updated reconnection model of the dayside solar windmagnetosphere interaction that does not predict net velocity enhancements along the sunward magnetopause. This model incorporates the observed (Hedgecock and Thomas, 1976) symmetric susp configuration of the outer polar cusp regions. Reconnection lines in this model are expected to emanate from the cusp regions instead of the subsolar region. Accelerated flows are not predicted along the dayside MPL because reconnection acts to decelerate and redirect the magnetosheath flow.

Crooker's model is bimodal vecause the HLBL would be primarily fed by reconnection processes near the cusp although the LLBL is considered to be primarily populated by nonreconnection processes. Thus, bimodal denotes the likely presence of more than one interaction process. The bimodal model is consistent with the observation that the HLBL thickness is correlated with IMF $B_{z}$ (Sckopke et al., 1976; Paschmann, et al.: 1976) whereas the LLBL thickness has not revealed a similarly clear correlation (Haerendel et al., i978; Eastman and Hones, 1978; see section 2.4 .5 for a possible correlation). This model does not specify a source mechanism for the LLBL. In general, the IMP 6 satellite is not in a position to test this model because observations in the HLBL and the cusp regicns are required. Observations of the HLBL and LLBL by Heos 2 (Rosenbauer et al., 1975; Haerendel et al., 1978) are consistent with the bimodal model. 


\subsection{Diffusion Due to Plasma Kinetic Instabilities}

The observations discussed in Chapter 2 indicate that the PBL is supplied by direct transport of magnetoshsath plasma across the MPL and that this transport is relatively widespread over the entire sunward magnetospheric boundary. A finite magnetic field component normal to the magnetopause surface (section 3.1.2) could account for some Darticle transport across the MPL. However, this mechanism cannot account for the presence of magnetosheath-like plasma on closed field lines in the PBL. These results suggest a diffusion mechanism and we will now consider whether an appropriate diffusion mechanism is available. For spatial diffusion of plasma,

$$
\frac{d n}{d t}=\nabla \cdot(D V n)
$$

where $D$ denotes the diffusion coefficient. A diffusive process is consistent with an overall decrease in density when passing earthward through the PBL with no isolated discontinuities in density. The near-noon crossing at $\lambda_{\mathrm{GSM}} \simeq 26^{\circ}$ by ISEE 1 (Figure 2.3.8.1) shows a sharp density drop at the MPL and at the inner surface of the PBL. The PBL in this case cannot be explained by local diffusion alone. However, the IMP 6 PBL crossings, which are farther from the subsolar point (see Figure 2.2.2), usually show no distinct change in density at the MPL or the inner surface of the PBL. In addition, the observed density has an overall decrease when passing earthward through the PBL. Significant small-scale fluctuations (section 2.4.2) are superimposed on this overall density decrease. Variations in plasma parameters as observed 
by IMP 6 are frequently consistent with a diffusion mechanism that transports plasma across the MPL into tine PBL.

Classical diffusion is characterized by a diffusion coefficient that is proportional to the electron-ion collision frequency ${ }_{c}$. For number densities near the MPL, $\nu_{c} \simeq 10^{-6} \mathrm{~s}^{-1}$ (i.e., essentially collisionless) and the classical diffusion coefficient is much too low to account for any significant diffusion. Furthermore, Gary (1979) has demonstrated that, for classical transport of a plasma with $B \sim 1$, the current layer and plasma density gradient should have comparable thicknesses. Thus, classical diffusion cannot explain the presence of a PBL.

In contrast to classical transport, diffusion caused by plasma kinetic instabilities can contribute plasma to a PBL. Gary (1979) has shown that the plasma diffusion rate across $B$ is determined by $n_{1}$, whereas the rate of cross-field penetration of a parallel current depends on $\eta_{\|}$where $\eta_{1}$ and $n_{\|}$denote the perpendicular and parallel resistivity, respectively. The mean ratio of PBL to MPL thickness $\left(\mathrm{L}_{\mathrm{PBL}} / \mathrm{L}_{\mathrm{MPL}}\right.$ ) observed by IMP 6 is $\simeq 18$ (section 2.4.4). If a diffusion mechanism is a major contributor of PBL plasma, the observed large ratio of PBL to MPL thickness requires that $n_{1} \gg n_{\|}$.

Density gradients perpendicular to the magnetic field can be a source of free energy that drive plasma microinstabilities. The enhanced field fluctutions that result can scatter particles and can lead to rates of cross-field plasma transport that considerably exceed classical values. Corresponding "anomalous" transport coefficients often satisfy $\eta_{\perp} \gg \eta_{\|}$(Gary, 1979). Field-aligned currents associated 
with the MPL could also be a source of free energy to drive microinstabilities.

IMP 6 observations of the MPL-PBL region indicate that

(1) $B \geq 1$,

(2) $T_{i} \gg T_{e}$,

(3) in general, $\mathrm{L}_{\mathrm{PBL}} \gg \mathrm{L}_{\mathrm{MPL}}$,

(4) the density gradient has a sign opposite to that of the magnetic field magnitude gradient and the temperature gradient,

(5) the density gradients are generally perpendicular to $\vec{B}_{0}$ where $\vec{B}_{0}$ denotes the ambient magnetic field, and

(6) the electron-ion relative drift speed $V_{D}<0.3 V_{i}$ where $V_{i}$ denotes the ion thermal speed.

Plasma wave observations in the MPL-PBL region by the ISEE satellites (Gurnett et al., 1979) show significant magnetic and electrostatic fluctuations at frequencies much greater than $\omega_{c}$. This paper reports broad-band electric fluctuations from a few $\mathrm{Hz}$ to $\wedge 100 \mathrm{kHz}$ and magnetic field turbulence from a few $\mathrm{Hz}$ to $n \mathrm{~g} \mathrm{kz}$ with maximum wave intensities in the MPL-PBL region. A polarization of $\overrightarrow{\delta E} \perp \vec{B}_{0}$ was also observed where $\delta \vec{E}$ denotes fluctuations of the electric field.

From these observations, the plasma microinstability that is probably dominant in the MPL-PBL region can be identified by a process of elimination. Instabilities that operate when $\beta\rangle_{\nu} 1$ and $T_{i} \gg T_{e}$ (with a current or gradient as a source of free energy) are the lower hybrid drift (LHD), the Alfuen drift, the ion cyclotron current, and the electromagnetic current instability (S. Peter Gary, private communications, 1979). For $V_{D} \leqslant 0.3 V_{i}$, as observed near the MPL, the 
ion cyclotron current instability is not excited (Forslund et al., 1978). At these $V_{D}$ values the electromagnetic "kink-like" current instability can grow, but it is a long-wavelength MHD-like mode that cannot enhance cross-field diffusion by the microscopic processes considered here (Gary et al., 1976) although it may be significant for processes involving large-scale inhomogeneities (see section 3.2.2). The Al fuen drift instability may be present; however, its growth rate is small compared to that of the LHD instability for plasma conditions observed in the MPL-PBL region.

Therefore, by elimination, the LHD instability is probably the most important microscopic instability in the MPL-PBL region. The LHD instability is driven by diamagnetic currents perpendicular to $\vec{B}$ (Davidson and Gladd, 1975). It propagates in the direction of the ion diamagnetic drift (i.e., $\vec{k} \perp \vec{B}_{0}$ ) with a characteristic wave number $k$ given by $k R_{e} \sim\left(T_{e} / T_{i}\right)^{1 / 2}$ (Lemons and Gary, 1978). The frequency of the LHD instability is not strictly at the lower hybrid frequency $\Omega_{\mathrm{LH}}$ $\left[=\omega_{p_{i}} /\left(1+\omega_{p_{e}}^{2} / \omega_{c}^{2}\right)^{1 / 2}\right]$; instead, it is proportional to the local diamagnetic drift speed and thereby proportional to the local cross-field density gradient. Considering the density fluctuations observed in the MPL-PBL region (section 2.4.2), a broad spectrum of waves should be excited by the LHD instability ranging from $\omega_{c}$ to above $\Omega_{\text {LH }}$ (S. Peter Gary, private communications, 1979). From Table 3.1.1 we note that $\omega_{c_{j}} \simeq 2 \mathrm{~s}^{-1}$ and $\Omega_{\mathrm{LH}} \cong 105 \mathrm{~s}^{-1}$, which comprises the low frequency range of the ISEE observations. The growti rate is enhanced for increasing values of $T_{i} / T_{e}$ and $\nabla n$ as well as for opposite signs for Pn and $\nabla T$ (Gary and Sanderson, 1979). Davidson et al. (1977) have 
studied the effect of finite $\beta$ on the LHD instability and have found that $i t$ is not readily stabilized for $T_{i} / T_{e} \sim 10$ (see Figure 7 of Davidson et al., 1977) even for $B>2$.

Applying available theoretical results (Liewer and Davidson, 1977; Davidson et al., 1977; Caponi and Krall, 1975; Davidson et al., 1978), the MPL-PBL region will now be evaluated as a possible locale for the L.HD instability. The effective collision frequency for the LHD instability is given by

$$
v_{L H D}=\sqrt{\frac{\pi}{2}}\left(1+\frac{\omega_{p_{c}}^{2}}{\omega_{c}^{2}}\right) \Omega_{L H}\left(\frac{v_{E}}{v_{i}}\right)^{2} \frac{\epsilon_{F}}{n m_{e} v_{E}^{2}:}
$$

(Liewer and Davidson, 1977, equation 1) where $V_{E}$ is the electron $\vec{E} \times \vec{B}$ drift speed, $v_{i}\left(=\left(2 k T_{i} / m_{i}\right)^{1 / 2}\right)$ is the ion thermal speed and $\epsilon_{F}\left(=\left\langle\delta E^{2}\right\rangle / 8_{\pi}\right)$ is the energy density in the fluctuating electric fields. Liewer and Davidson (1977) assumed that the currents are characterized by an electron $\vec{E} \times \vec{B}$ drift speed, $V_{E}$; however, their treatment was developed for rapidly pulsed theta pinch experiments in which the ions could be assumed as unmagnetized and the electrons as magnetized. Near the magnetopause, however, the ions will normally complete their gyromotions and the $\vec{E} \times \vec{B}$ drift will not result in a net current because the $\vec{E} \times \vec{B}$ drift affects both ions and electrons equally. Thus, the oppositely directed diamagnetic drift of ions and electrons,

$$
v_{D}\left(=\frac{c|\nabla P x \vec{B}|}{e B^{2}} \simeq v_{i}^{2} / 2 \omega_{c_{i}} L_{p}\right),
$$


will be the dominant source of free energy to drive the instability. For ions, $V_{D} \approx 62 \mathrm{~km} / \mathrm{s}$ using $L_{p} \approx 250 \mathrm{~km}$ for the pressure gradient scale length near the MPL (see section 2.4.2 and Table 3.1.1). The electron diamagnetic drift is substantially lower. $V_{D}$ may now be used in equation (1) in place of $V_{E}$. Using typlcal values of plasma parameters for the MPL (also applicable to the outer extent of the PBL) we obtain VLHD $\simeq 52 \mathrm{~s}^{-1}$. $\epsilon_{F}$ has been evaluated using a value for $\delta E$ of $3 \mathrm{mV} / \mathrm{m}$ (see Gurnett et al., 1979). The corresponding anomalous resistivity is then

$$
\eta^{\text {LHD }}=\frac{4 \pi \nu}{\omega_{p_{e}}^{2}}=1.04 \times 10^{-8} \mathrm{~s}
$$

(Liewer and Davidson, 1977, equation 41). This result is slightly larger than the effective resistivity (or viscosity) value obtained by Eviatar and Wolf (1968).

The observed increase in nominal PBL thickness, $\mathrm{L}_{\mathrm{PBL}}$, with arc length, $S$, from the subsolar point, measured along the magnetopause, is presented in Figure 2.4.5.2b. At the dawn-dusk meridian $\left(X_{G S M}=0\right)$ the values are $\left\langle L_{P B L}\right\rangle=0.5 R_{E}$ and $S \cong 16 R_{E}$. A typical bulk flow speed in the PBL, $|\vec{\forall}|$, is $200 \mathrm{~km} / \mathrm{s}$. If magnetosheath plasma can diffuse to the inner extent of the PBL before reaching the dawn-dusk meridian, the implied diffusion time is $\tau_{D} \simeq S /|\vec{V}|=510 \mathrm{~s}$.

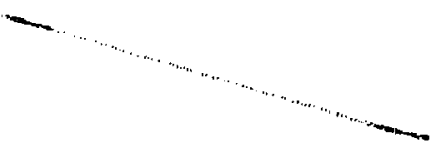


The diffusion time predicted for the LHD instability is found by using equation (2) above for $\eta_{\perp}$ in an expression derived by Gary (1979) for the density diffusion time.

$$
\tau_{\text {LHD }} \backsim \frac{8 \pi L_{p}^{2}}{n_{\perp c^{2} B}}=167 \mathrm{~s}
$$

A plasma $\beta$ of 10 is used for the MPL-PBL region (see Table 3.1.1). This approximate result suggests that the LHD instability could play a sig-

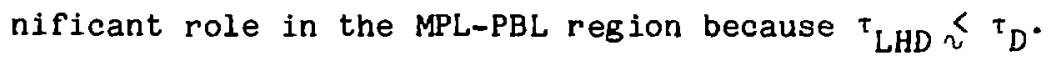

The LHD instability could be responsible for significant cross-field diffusion in the MPL-PBL region in the presence of a spatially limited current layer. This result is consistent with

(1) the observation of a PBL that is highly variable in thickness and comprises small-scale density fluctuations, and

(2) the observation of broad-band electrostatic emission and magnetic noise.

The last result is a consequence of the electromagnetic properties of drift wave instabilities (Davidson et al., 1977), which are particularly important at relatively long wavelengths (Lemons and Gary, 1977). 


\subsection{Review of Theory and Observations}

Because of the considerable complexities of the magnetopause region, determining the best theoretical description can most effectively proceed by a process of elimination. A review of the implications of satellite observations for various solar wind-magnetosphere interaction models is shown in the table below. This list should assist efforts in the falsification of certain theoretical descriptions and should thus provide some direction in the construction of improved theories.

EARLY MODELS (see section 3.2.1)

1. Two-fluid (cold plasma) models

2. Viscous fluid models
Non-tranfer models

No PBL included

No plasma transfer (only momentum transfer)

Density discontinuity expected across MPL

Discontinuity in spectra expected across MPL

High-speed outflow expected

PBL thickness should correlate with magnetosheath field orientation

Finite $B_{n}$ expected

PBL expected to be on open field lines
Transfer is very probable

PBL observed

Plasma transfer very probable

Frequent density continuity near the MPL

Spectra are almost always very similar across the MPL

No clear observations of high speed outflow have been made

Clearly observed only for HLBL

Frequently observed

Not consistent with available data on the LLBL 


\section{RECENT THEORIES (see section 3.2.2)}

1. Sporadic reconnection

2. Convective turbulence

3. Particle orbit theory

4. Impulsive penetration
Disordered flow field expected in $\mathrm{PBL}$

Local plasma heating expected near MPL

Sunward flow component often expected in PBL

Irregular flow pattern expected in cusp regions

Hardening of spectra expected immediately earthward of the MPL

Requires large scale inhomogeneities

Predicts $L_{\text {PBL }} \gg L_{M P L}$

Predicts significant cross-fleld plasma flow in $\mathrm{PBL}$

5. Diffusion via the lower hybrid drift instability
Requires small-scale inhomogeneities

Predicts wave frequencies $>\omega_{c_{i}}$ and $\vec{k} \perp \overrightarrow{\mathrm{B}}_{0}$

Predicts the presence of a PBL
Disordered flow field observed only in the cusp regions and the subsolar region

Not clearly observed by IMP 6

Sunward flow component in PBL rarely observed outside cusp regions.

Irregular flow observed in cusp regions and also in subsolar region by ISEE

Not clearly observed

Large-scale inhomogeneities probable

Observed

often observed

Frequently observed

Observed

Observed 
CHAPTER 4

CONCLUSIONS

The objective of this thesis has been the identification of the primary plasma source for the plasma boundary layer (PBL) of the magnetosphere. Using the IMP 6 low-energy plasma data set with a time resolution in some parameters as short as $n 3.1 \mathrm{~s}$, I have used both a case-by-case and a statistical study to provide detailed descriptions of PBL and MPL characteristics. This analysis has demonstrated that

(1) PBL plasma within a few ion gyroradii of the MPL is often indistinguishable in density and spectra from the nearby magnetosheath plasma and, consequently, must be supplied by magnetosheath plasma by transport across the MPL at positions that are not far removed from the points of observation. IMP 6 measurements indicate that this transport process is relatively widespread over the entire sunward magnetospheric boundary.

(2) Magnetosheath plasma provides the major source of all PBL plasma although some magnetospheric plasma is steadily mixed with the magnetosheath plasma. Thus, the PBL is a mixing region that separates two plasma regimes.

(3) The PBL thickness is highly variable and, in general, $L_{P B L} \gg$ $\mathrm{L}_{\text {MPL }}$ Observed PBL signatures indicate that it is supplied by a highly variable process, both temporally and spatially.

(4) The low-latitude portion of the PBL (or LLBL) is located in a quasi-trapping region that contains predominantly closed field lines. Significant cross-field flow of plasma is frequently 
observed, indicating that the PBL can act as an MHD generator for field aligned currents linking it to the cusp ionosphere.

(5) The observed decrease in energy density, combined with the similarity in energy spectra, in passing from the magnetosheath through the LLBL suggests that plasma energy is dissipated in the PBL.

Observation (3) is incompatible with a classical diffusion process that would diffuse the current and density sheaths at approximately the same rate so that the PBL could not be distinguished from the MPL. A brief analysis of the lower hybrid drift instability indicates that it can be a significant contributor to the PBL. Furthermore, such a density gradient driven instability can lead to significant cross-field diffusion independent of the local magnetosheath field orientation. Considering the variability of the impinging magnetosheath plasma flow, cross-field diffusion by way of the lower hybrid drift instability could explain many observed PBL characteristics.

Observations (4) and (5) are inconsistent with the presence of reconnection processes, not excluding reconnection processes in the cusp regions that can subsequently supply the HLBL (or plasma mantle). The bimodal model of Crooker (1977) and the convective turbulence model of Haerendel (1978) are promising theoretical descriptions that are not necessarily inconsistent with available IMP 6 observations (which are primarily limited to the LLBL). In fact, occasional reconnection processes are supported by accelerated flow observed by IMP 6 in two cusp crossings (Fairfield and Hones, 1978). 
A comparison of theoretical descriptions of the solar windmagnetosphere interaction illustrates the problems posed for all theoretical models. The most significant problems are the high spatial and temporal variations observed near the magnetopause. Realistic models of the magnetopause interaction must account for the presence of the PBL and account for transport of magnetosheath plasma across the MPL. 
APPENDIX A

IMP 6 INSTRUMENTATION AND ANALYSIS TECHNIQUES

\section{A1. Electrostatic Analyzer}

The Los Alamos Scientific Laboratory (LASL) electrostatic analyzer on board IMP 6 is the hemispherical-plate type [discussed in detall by Theodoridis and Paolini (1969) and Vasyluinas (1971)]. The analyzer accepts particles through an entrance aperture with an azimuthal acceptance angle $\Delta \phi\left(=2.41^{\circ}\right)$ and a range of polar angles from ${ }^{-\theta_{m}}$ to $\theta_{\mathrm{m}}$. Thus, the long dimension of the analyzer fan is parallel to the spin axis. For a given electrostatic potential between the two concentric hemispherical plates, particles that reach the detect or have a limited energy range $\Delta E_{i}$ centered on 16 discrete energy values $E_{\mathbf{i}}$. Sweeps through the various energies are accomplished by bringing the plate potential up to an intial high level and then exponentially decreasing the potential (hence, the accepted range of particle energies). Analyzer parameters are summarized in Table A1.

The IMP 6 satellite is spin-stabilized with the spin vector directed toward thə south ecliptic pole. Ions and electrons are sampled alternately four times per spin period, so that in eight rotations ( $s 100 \mathrm{~s}$ ), 32 equally spaced azimuthal angles are sampled for each charge sign. Thus, energy spectra are obtained for both ions and electrons every s $3.1 \mathrm{~s}(1 / 4$ of a spin period). Table A2 lists the 16 logarithmically spaced energy values sampled, which range from 140 ev to $28.8 \mathrm{keV}$ for ions and from $13.3 \mathrm{eV}$ to $18.1 \mathrm{keV}$ for electrons. 
Table A1. Electrostatic Analyzer Parameters

\begin{tabular}{|c|c|c|c|}
\hline & \multirow{2}{*}{$\begin{array}{l}\text { Parameter } \\
\text { Description }\end{array}$} & \multicolumn{2}{|c|}{ Parameter Values } \\
\hline Parameter & & Protons & Electrons \\
\hline$\Delta \phi$ & $\begin{array}{l}\text { azimuthal acceptance } \\
\text { angle (degrees) }\end{array}$ & 2.41 & 2.41 \\
\hline$\theta_{m}$ & $\begin{array}{l}\text { hal } \mathrm{f} \text {-width of polar } \\
\text { acceptance angle (degrees) }\end{array}$ & 45.0 & 45.0 \\
\hline$\gamma$ & $\begin{array}{l}\text { relative width of energy } \\
\text { window, } \Delta E / E\end{array}$ & 0.0217 & 0.0217 \\
\hline$\alpha$ & ratio of $E_{i}$ to $E_{i-1}$ & 1.426 & 1.613 \\
\hline$\tau$ & $\begin{array}{l}\text { integration time at each } \\
\text { sample point (s) }\end{array}$ & 0.02 & 0.03 \\
\hline $\mathbf{a}$ & area of aperture $\left(\mathrm{cm}^{2}\right)$ & 0.0476 & 0.0476 \\
\hline
\end{tabular}

Table A2. Energy Levels Sampled by the LASL Electrostatic Analyzer

\section{Energy \\ Channel}

1

2

3

4

5

6

7

8

9

10

11

12

13

14

15

16

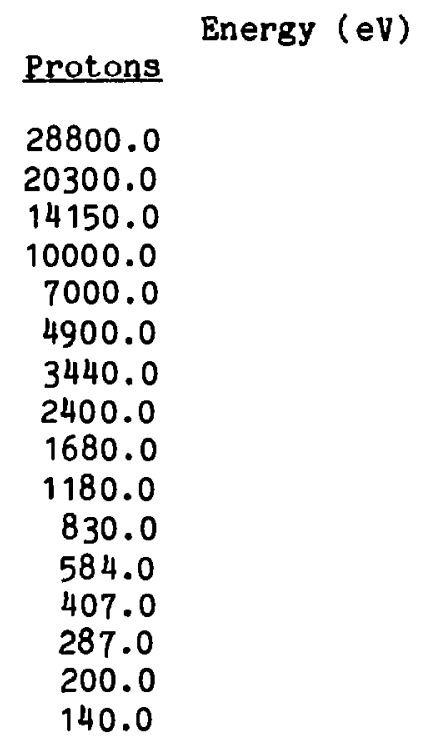

18100.0

10900.0

6450.0

3880.0

2330.0

1435.0

880.0

545.0

340.0

212.0

130.0

82.3

51.5

32.7

20.7

13.3 
Energy-angle count arrays sampled by IMP 6 represent an approximate twodimensional cross section of velocity space, although the $\pm 45^{\circ}$ analyzer fan samples about $79 \%$ of the full sphere in velocity space with the polar regions deleted. Complete 32-angle, 16-energy level velocity distributions are obtained approximately every $100 \mathrm{~s}$ although a rough (4-angle) velocity distribution is obtained from the ion measurements for every spin period ( $\sim 12.5 \mathrm{~s}$ ).

A2. Analysis Techniques

The counting rate $c(\vec{r}, \vec{v})$ of the detector for a given measurement location and velocity can be derived in terms of known parameters and the distribution function $f(\vec{r}, \vec{v})$ or phase space density $\left(\# / \mathrm{cm}^{6} s^{-3}\right)$. $f(\vec{r}, \vec{v})$ can then be solved in terms of these known parameters and the measured counting rate. A detailed description of this analysis technique as it is applied to data from LASL electrostatio analyzers on the IMP satellites is given by Stiles (1976). If we denote the magnitude of $\vec{v}$ by $v$ and drop the position vector, $\vec{r}$, the differential flux $j$ is given by

$$
j(\vec{v})=\vec{v} f(\vec{v}) d \vec{v} \text {, and }[j]=\# / \mathrm{cm}^{2}-s^{1} \text {, }
$$

where the units of $j(\vec{v})$ are represented by $[j]$.

In spherical coordinates $j(\vec{v})$ can be written as

$$
j(\vec{v})=v f(v, \phi, \theta) v^{2} d v \cos \theta d \phi d \theta \text {. }
$$

If we use energy in place of velocity, using $E=\frac{1}{2} m v^{2}$, this becomes

$$
f(E, \phi, \theta)=\frac{2}{m^{2}} f(E, \phi, \theta) E d E \cos \theta d \theta d \phi,
$$

and the differential flux per unit solid angle $(d \Omega=\cos \theta d \theta d \phi)$ is 


$$
j^{\prime}=\frac{2}{m^{2}} \operatorname{Er}, \quad\left[j^{\prime}\right]=\# / \mathrm{cm}^{2} \cdot s \cdot s r \cdot E \text {. }
$$

The differential counting rate $d c_{i k}$ represents the number of plasma particles entering the analyzer during time $\tau$ through an aperture of area $a$. These particles have energy $E_{j}$ and enter the analyzer from the location $\left(\phi_{k}, \theta\right)$.

$$
d c_{i k}=\tau a \cos \theta j \cdot\left(E_{i}, \phi_{k}, \theta\right) d E_{i} \cos \theta d \theta d \phi_{k}
$$

The total number of particles sampled at energy $E_{i}$ and location $\phi_{k}$ is then found by integrating over the angular ranges of acceptance. If $f(E, \phi, \theta)$ is assumed to be approximately constant over each energy window and is independent of $\phi$ and $\theta$, then the integration results in

$$
c_{i k} \simeq \frac{4 \tau a \Delta \phi \sin \theta m_{i}^{\Delta E_{i} E_{i}}}{m^{2}} f\left(E_{i}, \phi_{k}, \theta\right) .
$$

Three main sources of error affect this result.

(1) The detectors respond to the higher energy cosmic ray background. The average number of cosmic ray counts per sample is independent of energy or angle and is simply subtracted from the measured counts.

(2) Photoelectrons produced by sunlight impinging on the surface of the satellite can add significantly to low-energy $(<100 \mathrm{eV})$ electron counts. Photoelectron corrections are routinely made in IMP 6 plasma analysis.

(3) The particle counter efficiencies depend on energy and were 
measured experimentally before launch. All measured counts are corrected by these efficiency factors.

The distribution function is then given approximately by

$$
f\left(E_{i}, \phi_{k}\right)=\frac{m^{2} C_{i k}}{4 \pi a \Delta \phi \sin \theta_{m} E_{i} \Delta E_{i}}
$$

where $\mathrm{C}_{i k}$ includes any appropriate corrections. Various plasma parameters are now calculated by taking velocity moments of this distribution function in terms of measured quantities. One of these moments is the pressure tensor from which "temperature" is derived. This temperature does not imply a condition of thermal equilibrium or a Maxwellian velocity distribution.

A3. Evaluation of Errors and Accuracy

Errors resulting from counting statistics are calculated by assuming that the samples are taken from a normal distribution and that the standard deviation of the mean for each measurement of counts $C_{i k}$ is given by $C_{i k}{ }^{1 / 2}$. Counts for different values of $i$ and $k$ are assumed to be independent.

Errors caused by counting statistics for several plasma parameters are shown in Figure $A 1$ as a function of density. For typical magnetosheath and PBL density values, the relative errors are generally less than .05 to 0.1 .

A simulation of the count array $C_{i k}$ is used to evaluate the accuracy of the analysis procedure. A program was developed by G. S. Stiles for IMP 8, and subsequently modified by me for IMP 6 , to perform this simu- 


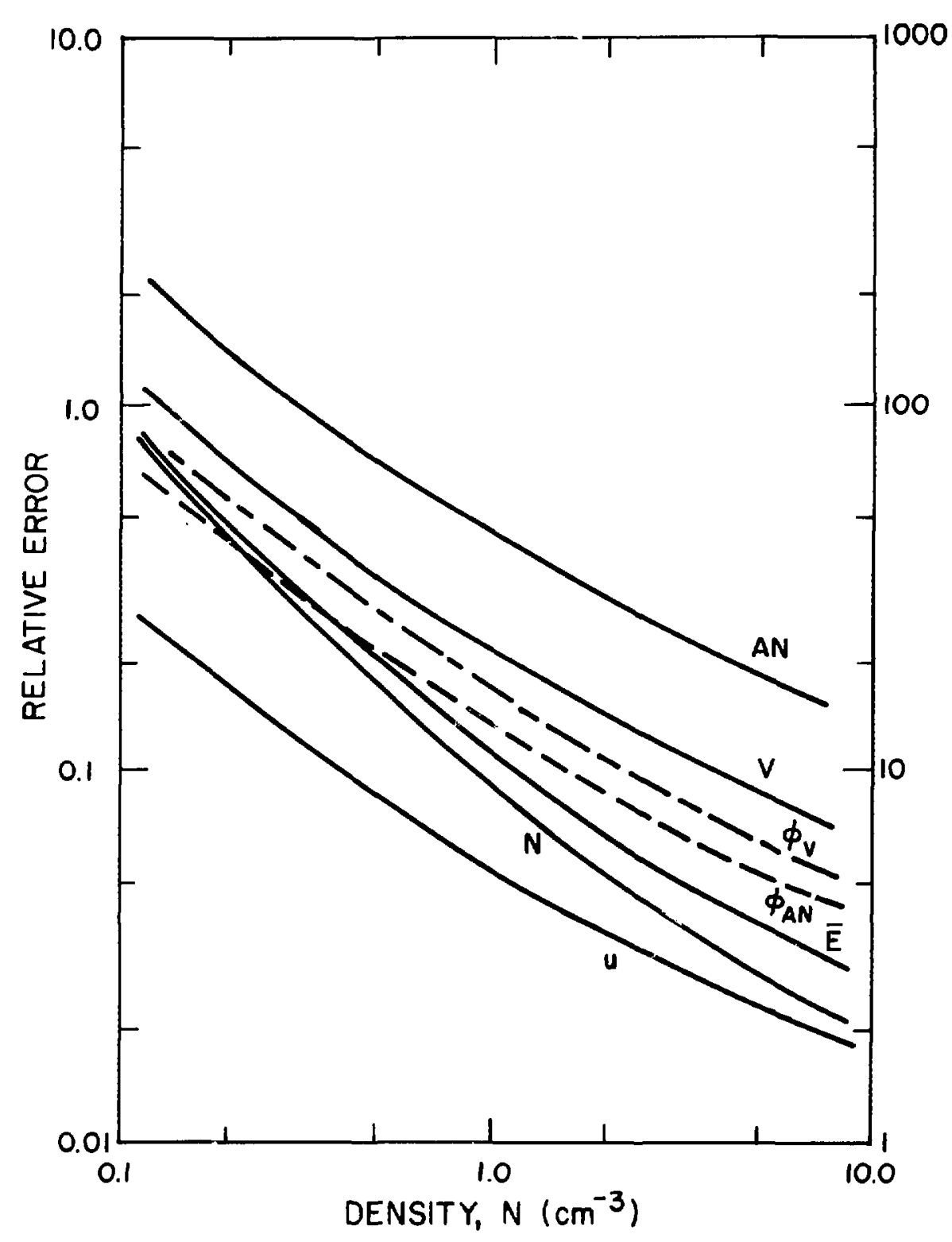

Figure A1 Errors due to counting statistics as a function of density. $N$,density; $\bar{E}$, mean thermal energy; $u$, energy density; $V$, velocity magnitude; AN, anisotropy magnitude; $\phi_{V}$, velonity angle; $\phi_{A N}$, anisotropy angle (angles are read on the right scale). 
lation. The program evaluates errors produced by the assumption that $f$ is independent of polar angle and that $f\left(E_{i}, \phi_{i k}\right)$ accurately represents the average of $f$ over the logarithmic energy interval centered on $E_{i}$ and the azimuthal interval of angle between samples.

Variations in several plasma parameters as a function of total energy are plotted in Figure A2. Perfectly accurate analysis would result in a value of 1.0 for a given parameter at a given energy. Values above or below 1.0 represent anomalously high or low measured values, respectively.

Variations for several plasma parameters as a function of polar angle of the "true" velocity vector are plotted in Figure A3. The ecliptic plane component for all parameters is measured very accurately; however, the velocity and anisotropy are significantly underestimated for polar angles of flow above $\sim 30^{\circ}$.

The analysis procedure is generally accurate to at least $10 \%$ over (1) an average energy range of $s 400 \mathrm{eV}$ to $\sim 6 \mathrm{keV}$, (2) a range of velocities from 0 to $n 500 \mathrm{~km} / \mathrm{s}$ and (3) over a range of energy distributions such that the average energy along any given axis does not exceed twice that along any other axis. 


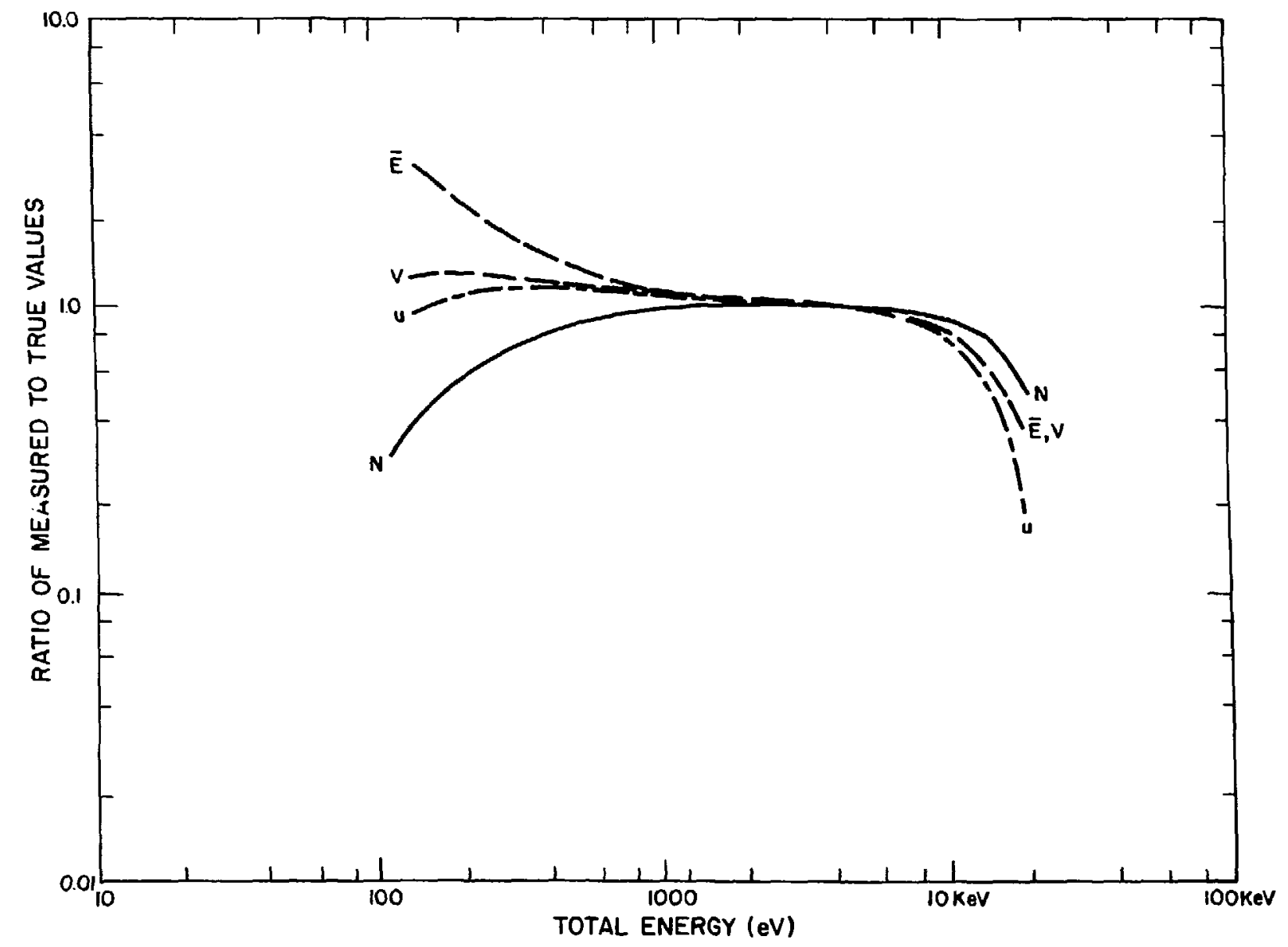

Figure A2 Variations of plasma parameters with total energy. The velocity distribution is assumed isotropic in the rest frame of the moving plasma which has a speed of $100 \mathrm{~km} / \mathrm{s}$. 


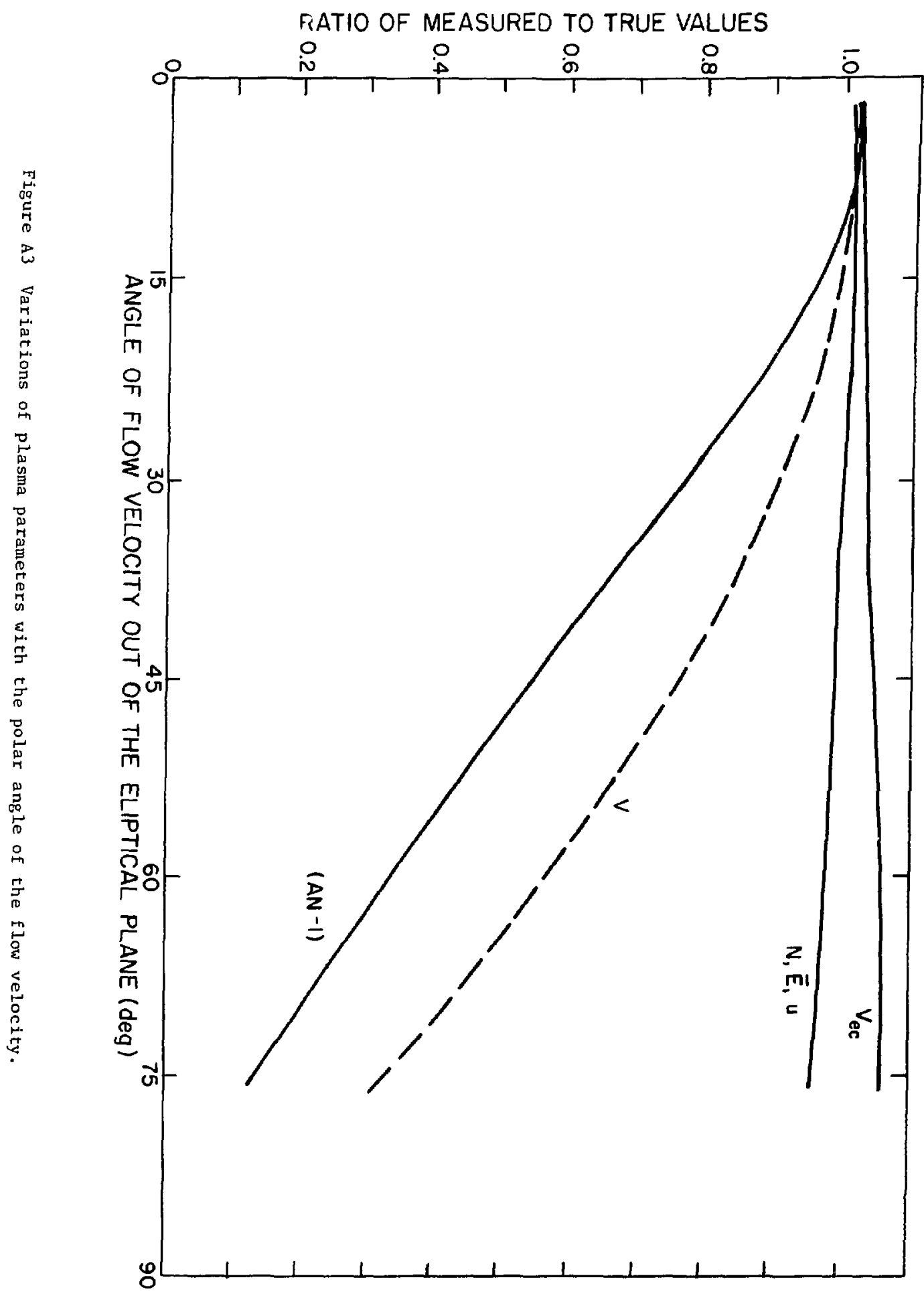




\section{APPENDIX B}

\section{SUPPLEMENTARY IMP 6 PBL EXAMPLES}

This appendix provides data on 20 additional IMP 6 MPL-PBL crossings with brief commentary for each crossing. The text (especially section 2.3) should be consulted for detailed descriptions of the data as applied to other crossing examples. Table 2.3 in the text lists the crossings that are presented.

\section{July 1971 IMP 6 Crossing}

This dawn-side PBL crossing shows no clear MPL signature. Selection of the outer position of the PBL is somewhat arbitrary. The location at 1318 UT marks the beginning of the decrease in plasma density to the magnetospheric level as well as the cessation of significant energetic electron intensity. However, the energetic electrons are not a reliable indicator of boundary positions. Enhanced ion anisotropy in the PBL and nearby magnetosheath is accompanied by a clear magnetosheath plasma depletion signature consistent with the presence of a mirror instability (Crooker et al., 1978). The inner PBL surface is no more clearly distinguished than in most other IMP 6 crossings. Only the cessation of magnetosheath-like bulk plasma flow and magnetic field fluctuations provides a check of its location. The bulk plasma flow may have some cross-field component based on the z-component of the field; however, the z-component of the plasma flow cannot be determined by the electrostatic analyzer (see Appendix A). Similar to many other PBL examples, the magnetic field direction in this crossing indicates a substantial tailward draping near the magnetospheric boundary. 


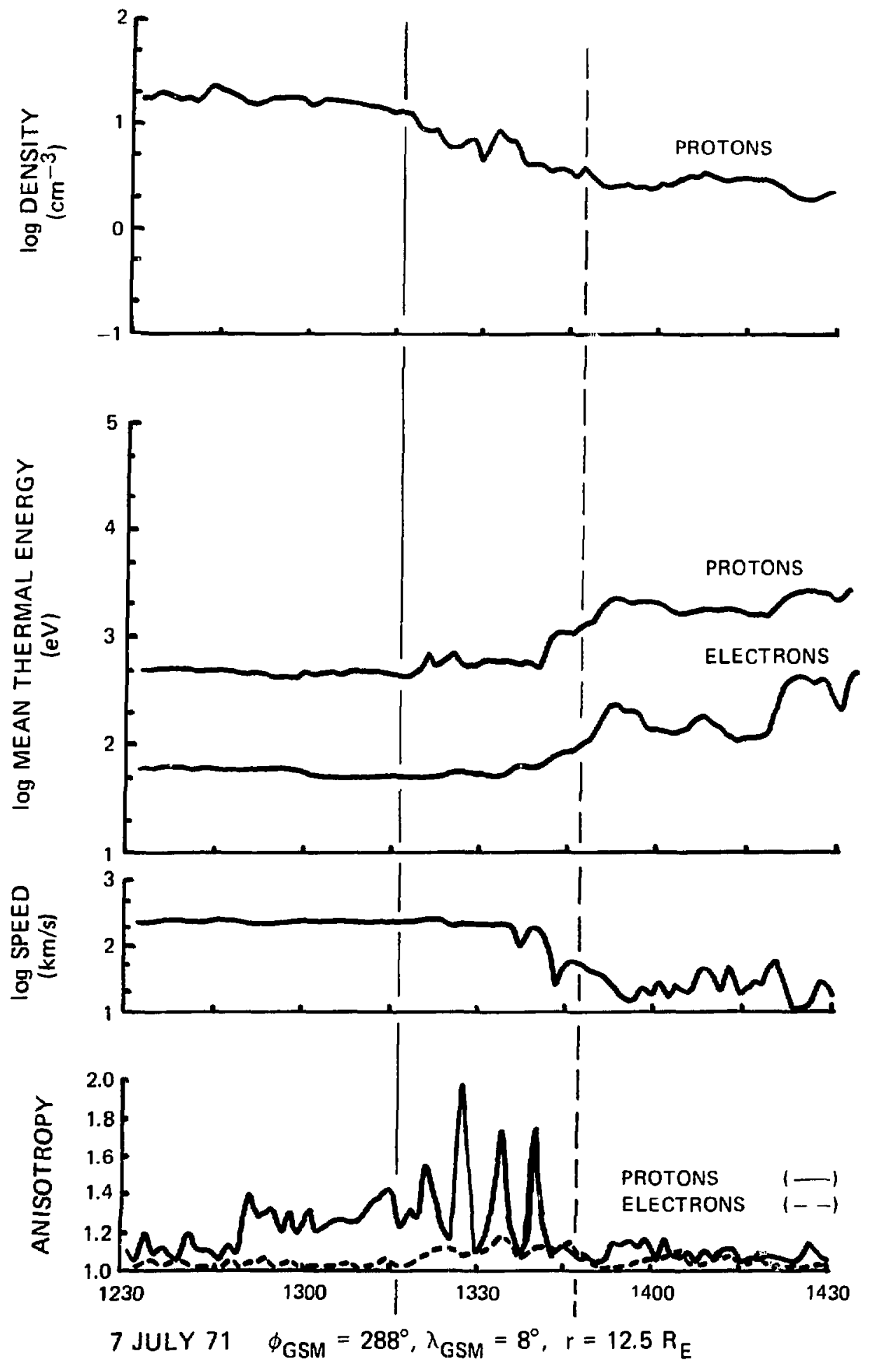




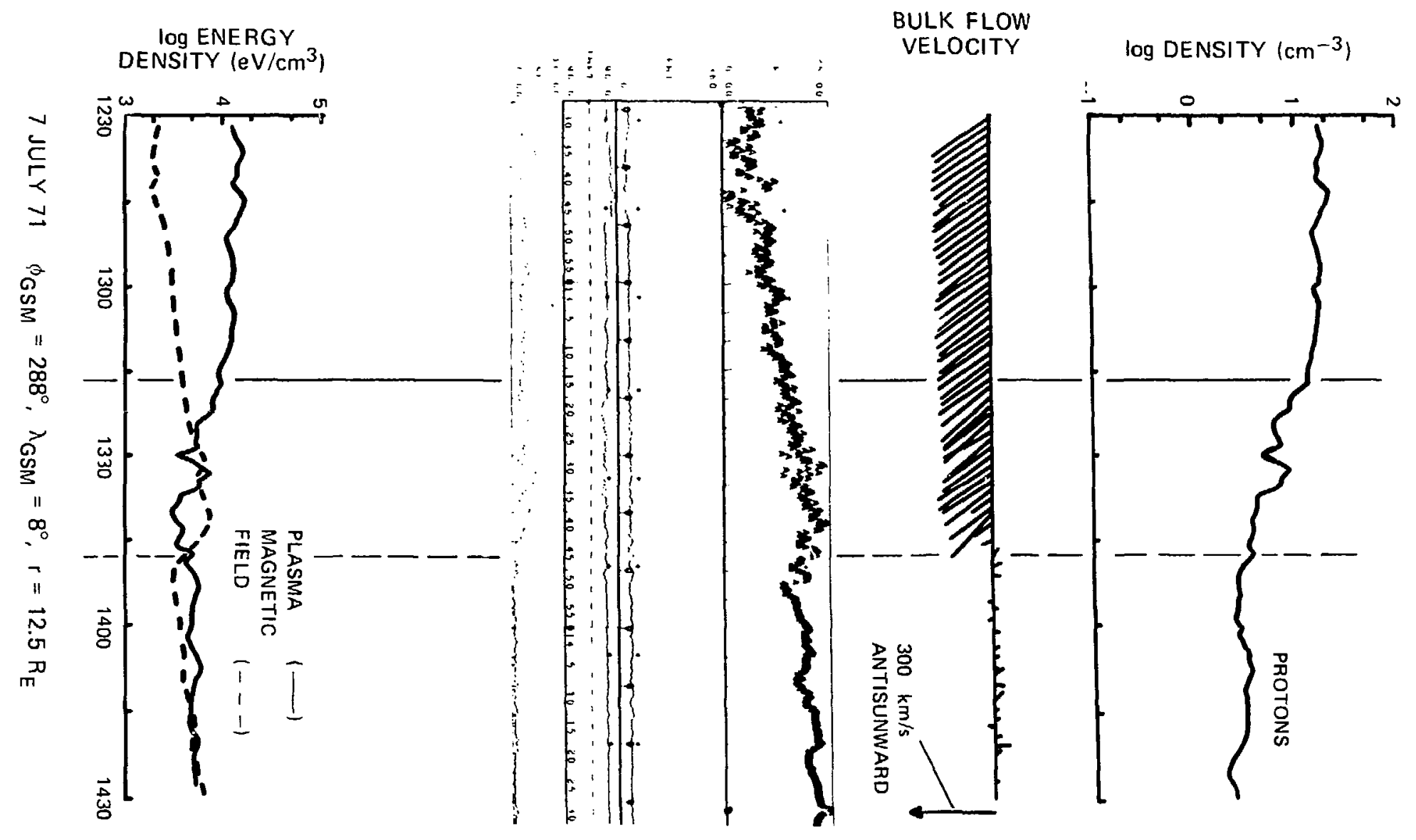


BULK FLOW AND MAGNETIC FIELD VECTORS 7 JULY 71

$$
30 \gamma \downarrow \prod_{\substack{300 \mathrm{~km} / \mathrm{s} \\ \text { ANTISUNWARD }}}^{T}
$$

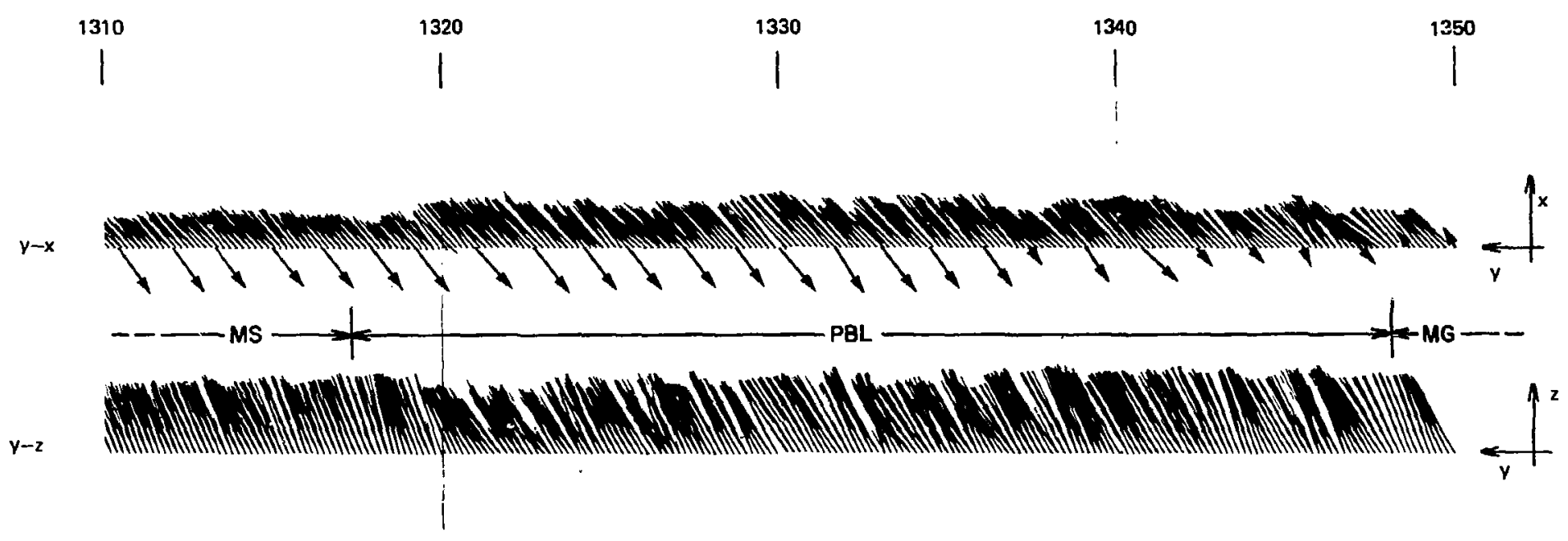


22 November 1971 IMP 6 Crossing

This dusk-side crossing near the dawn-dusk meridian shows a region of antisunward flowing plasma sampled more than one hour before the PBL. Isolated regions of sustained antisunward $\mathrm{r}^{\circ} \mathrm{l}, \mathrm{W}$ are often observed along the flanks of the magnetosphere far away fro'a the subsolar point (e.g., see al so the 5 January 1972 IMP 6 crossing in this appendix). The isolated region near 0315 UT may be caused by an earlier entry and exit of the PBL; however, the relative plasma characteristics and field direction are not fully consistent with this interpretation.

The intermediate mean thermal energy values observed in the PBL indicate that more magnetospheric plasma is mixed with the PBL at this location than is usually observed closer to the subsolar region. This commonly observed feature suggests that the entry mechanism for magnetosheath plasma into the PBL is less efficacious with increasing tailward distance.

Observed bulk plasma flow has a significant cross-field component throughout the PBL, although plasma flow in the detached region near 0315 UT has larger directional fluctuations and does not have a significant cross-field component. 


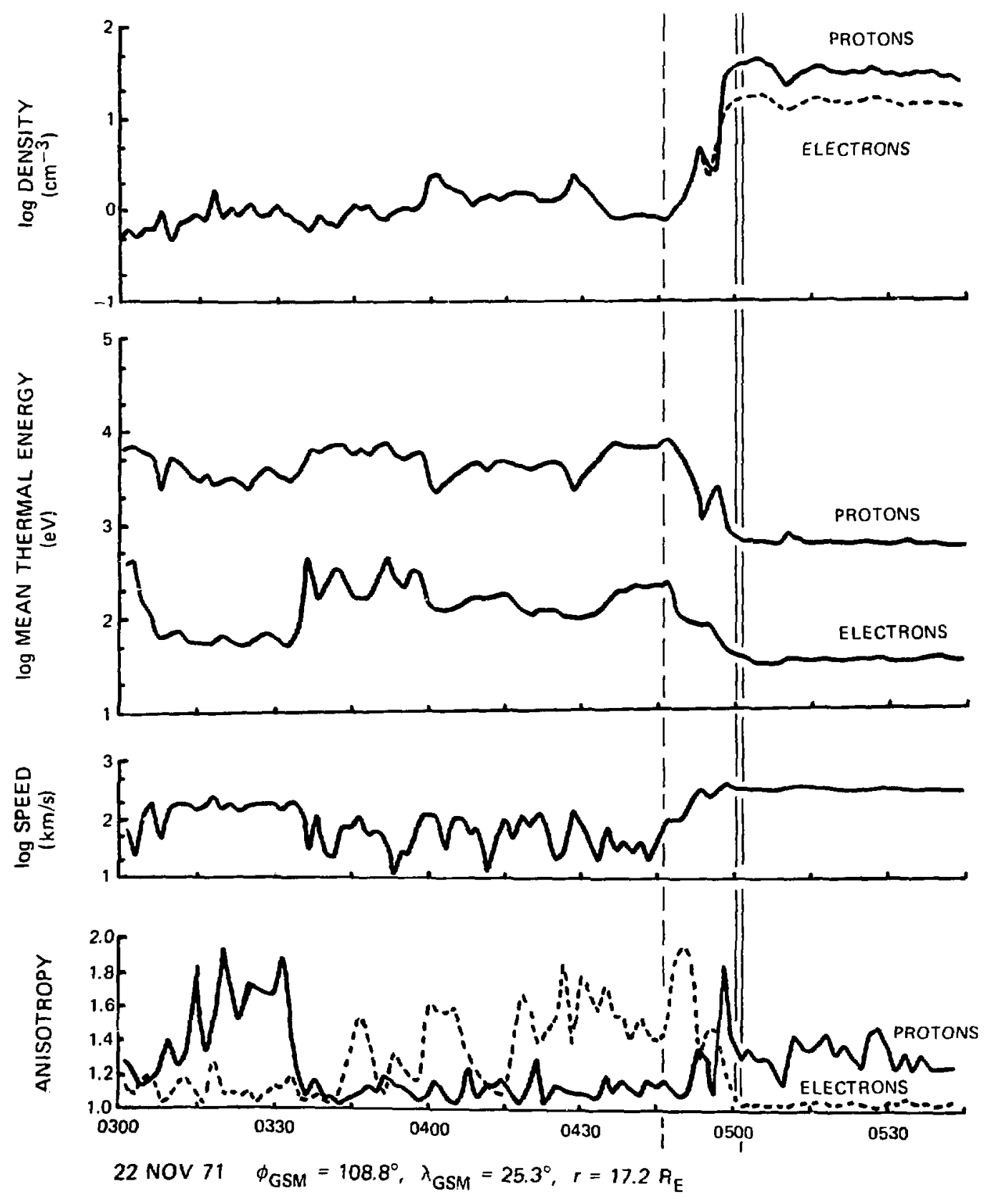




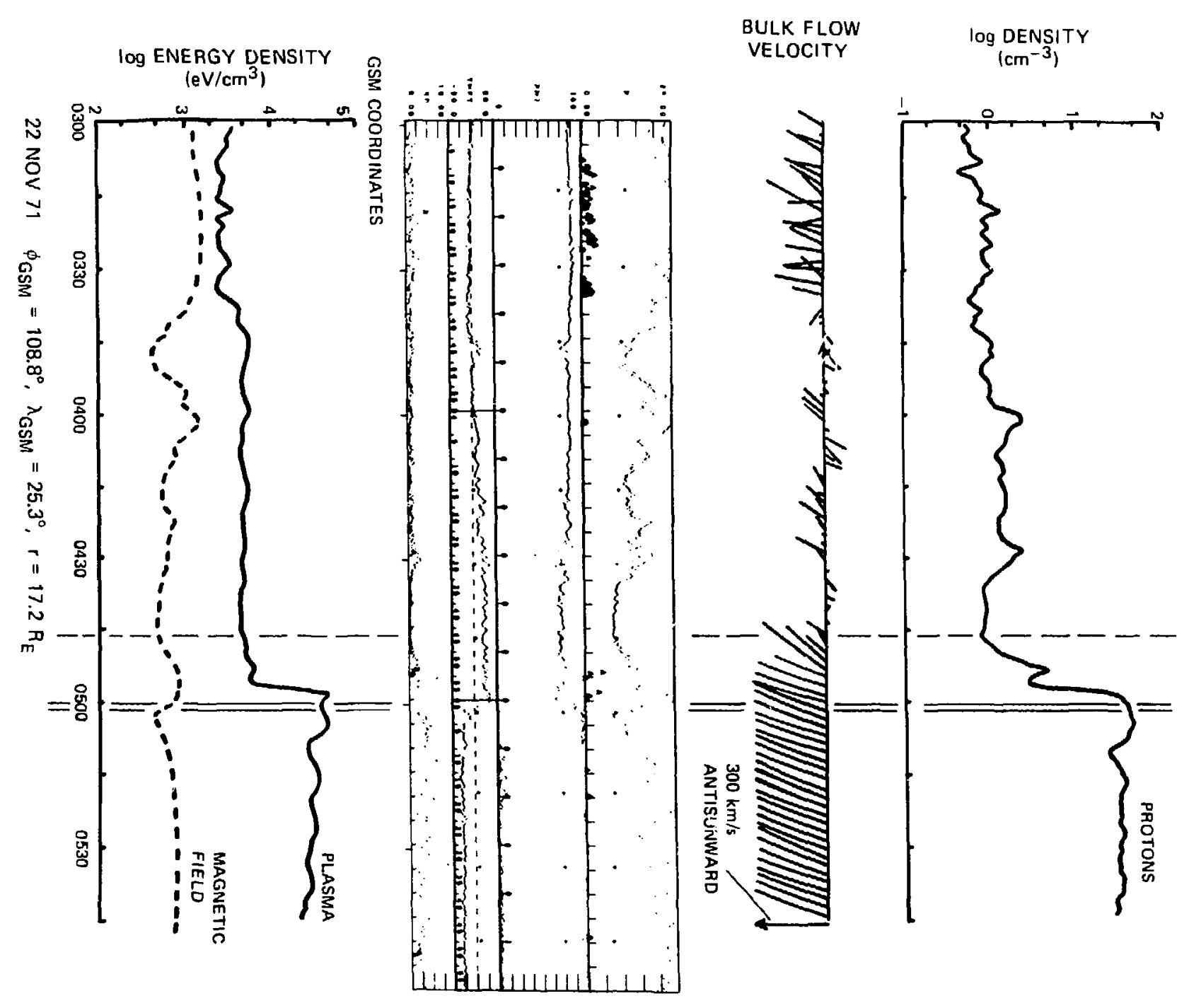



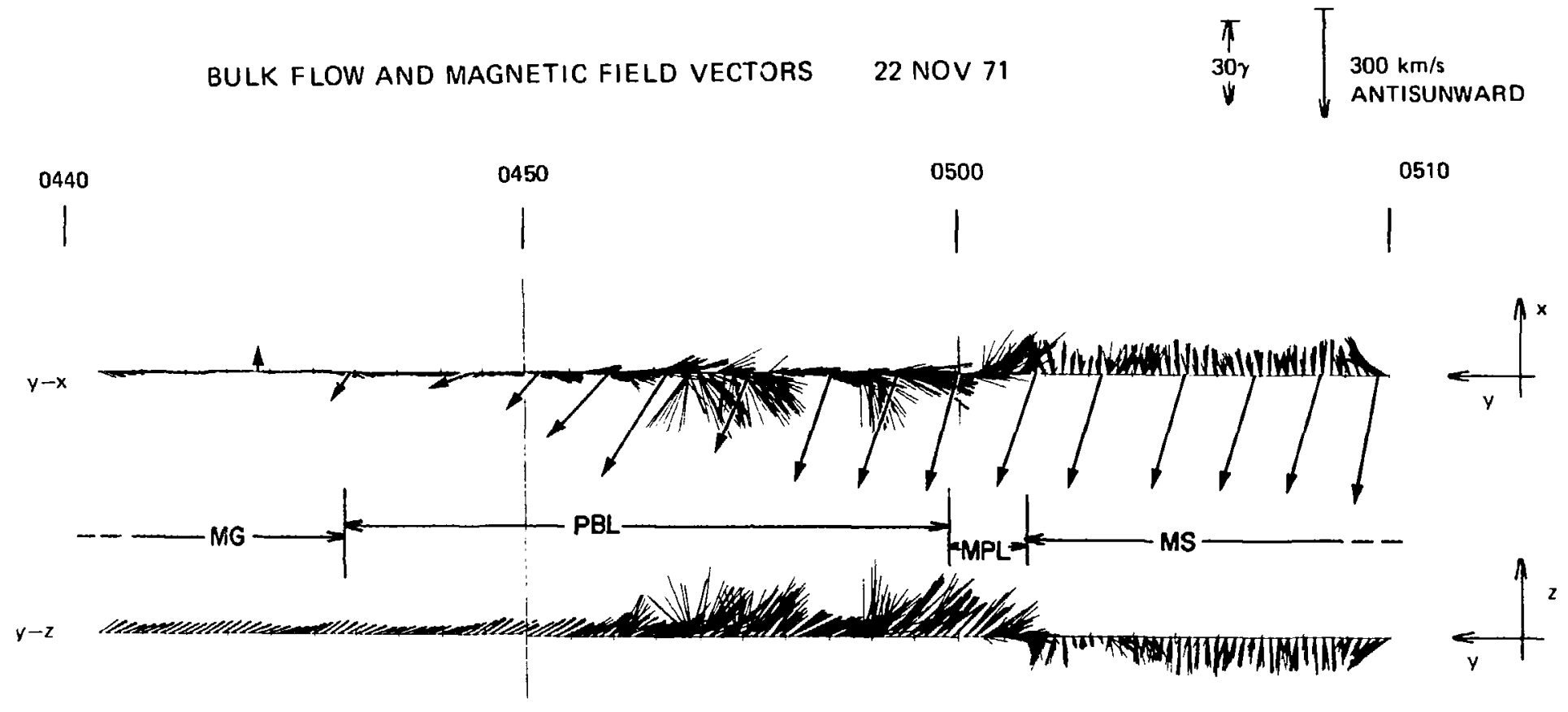
16 December 1971 IMP 6 Crossing

This higk-latitude dusk-side IMP 6 crossing does not have a clear MPL, although a possible location is marked near 2000 UT. The radial distance is only $9 R_{E}$ and corresponding large field magnitudes are observed. Field direction and location indicate a position immediately duskward of the outer cusp region with significant tailward draping of field lines in the PBL. Enhanced field fluctuations occur in the PBL that sometimes exceed the field magnitude.

An unusual sunward component flow vector appears in the PBL at 1915 UT. The PBL bulk flow field has enhanced directional fluctuations with high anisotropies cbserved in the PBL and nearby magnetosheath. These characteristics are common in the outer cusp region. 

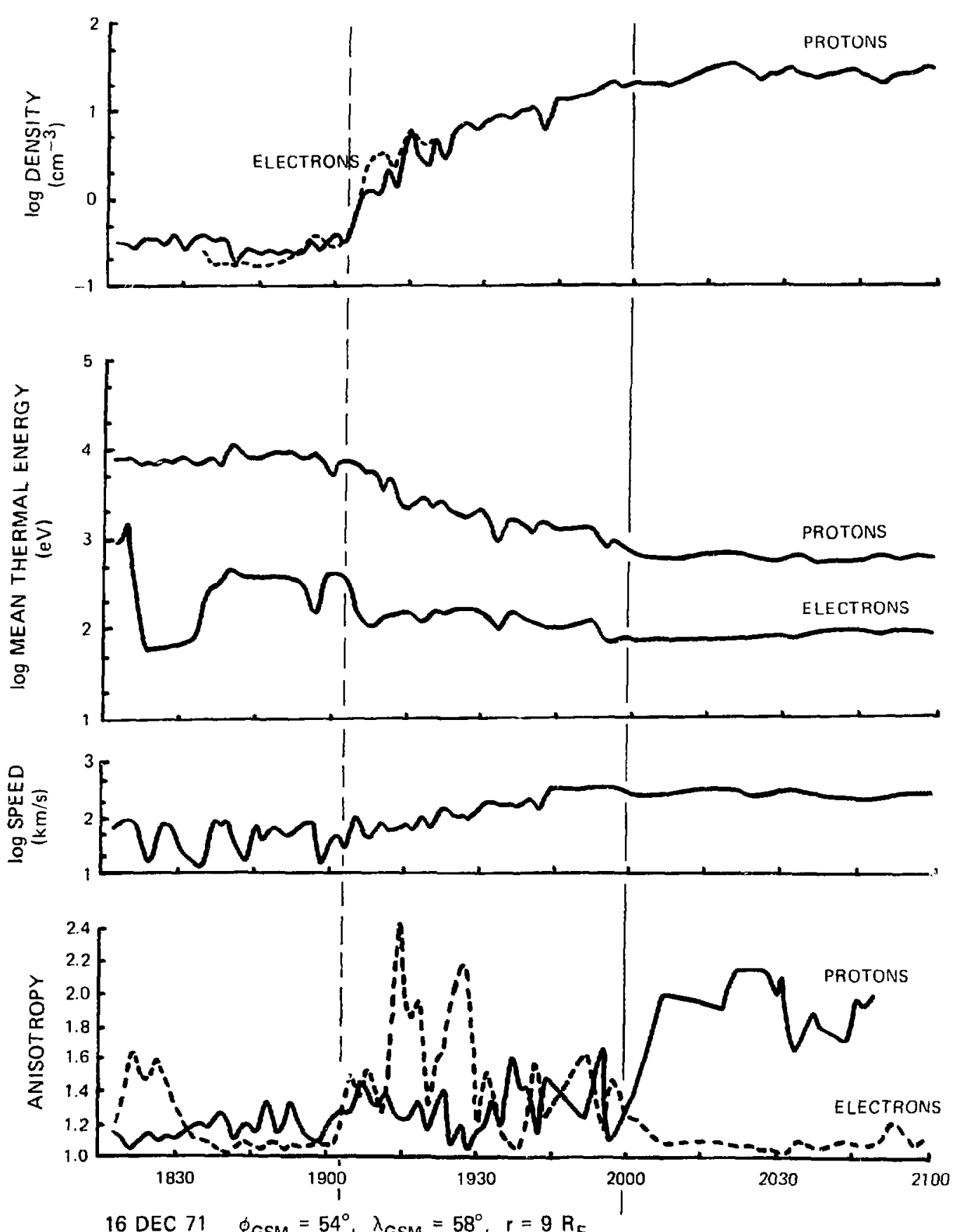

16 DEC $71 \phi_{G S M}=54^{\circ}, \lambda_{G S M}=58^{\circ}, r=9 R_{E}$ 

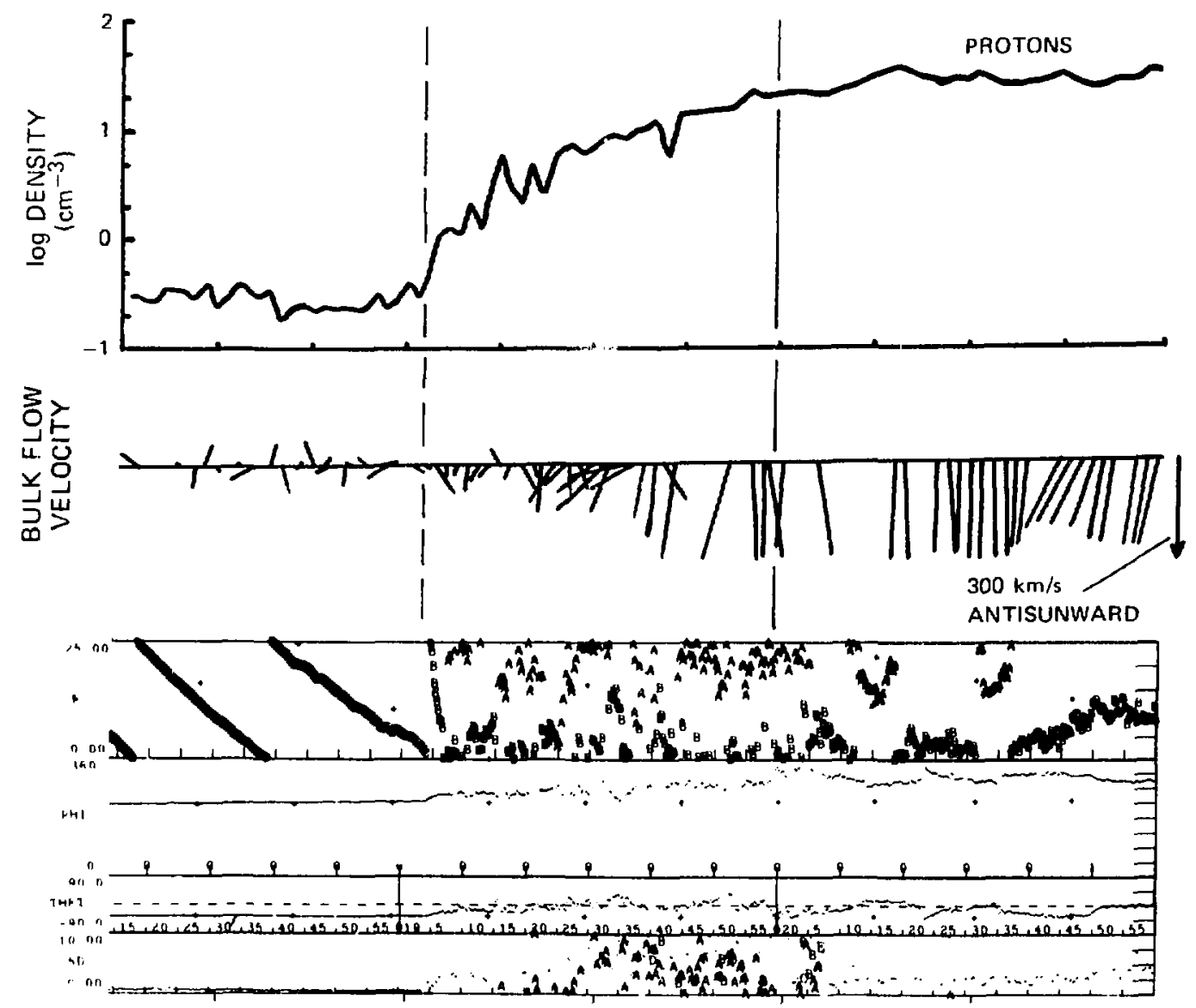

GSM COORDINATES

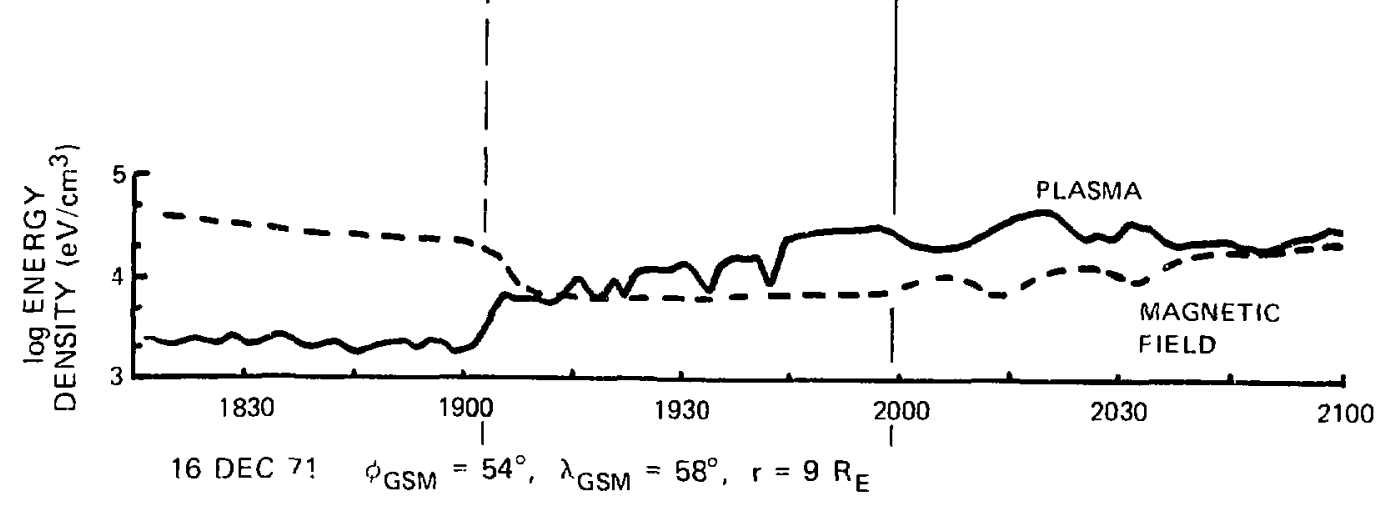




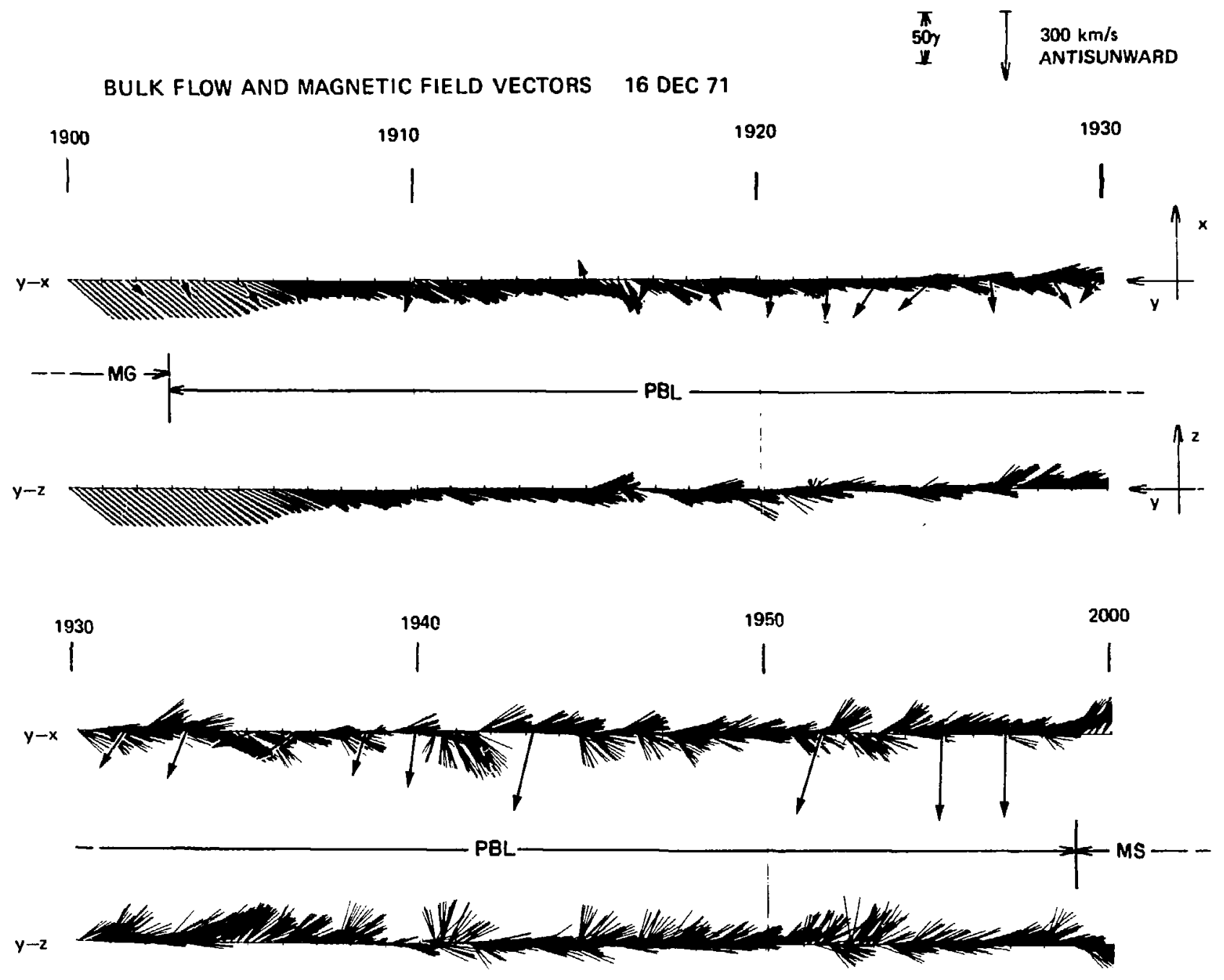


28 December 71 IMP 6 Crossing

Thiis dusk-side crossing is located near the equator tailward of the dawn-dusk meridian. It includes two complete MPL-PBL crossings; a rare circumstance for crossings of the sunward magnetospheric boundary but more common with increasing tailward distance. This difference results from larger fluctuations of the tailward magnetopause position relative to the sunward magnetopause position. The MPL and PBL are clearly present in all three marked crossing positions. Enhanced anisotropy values partly identify the PBL locations. Plasma flow in the PBL is generally within $\Omega 40^{\circ}$ of the field direction. 


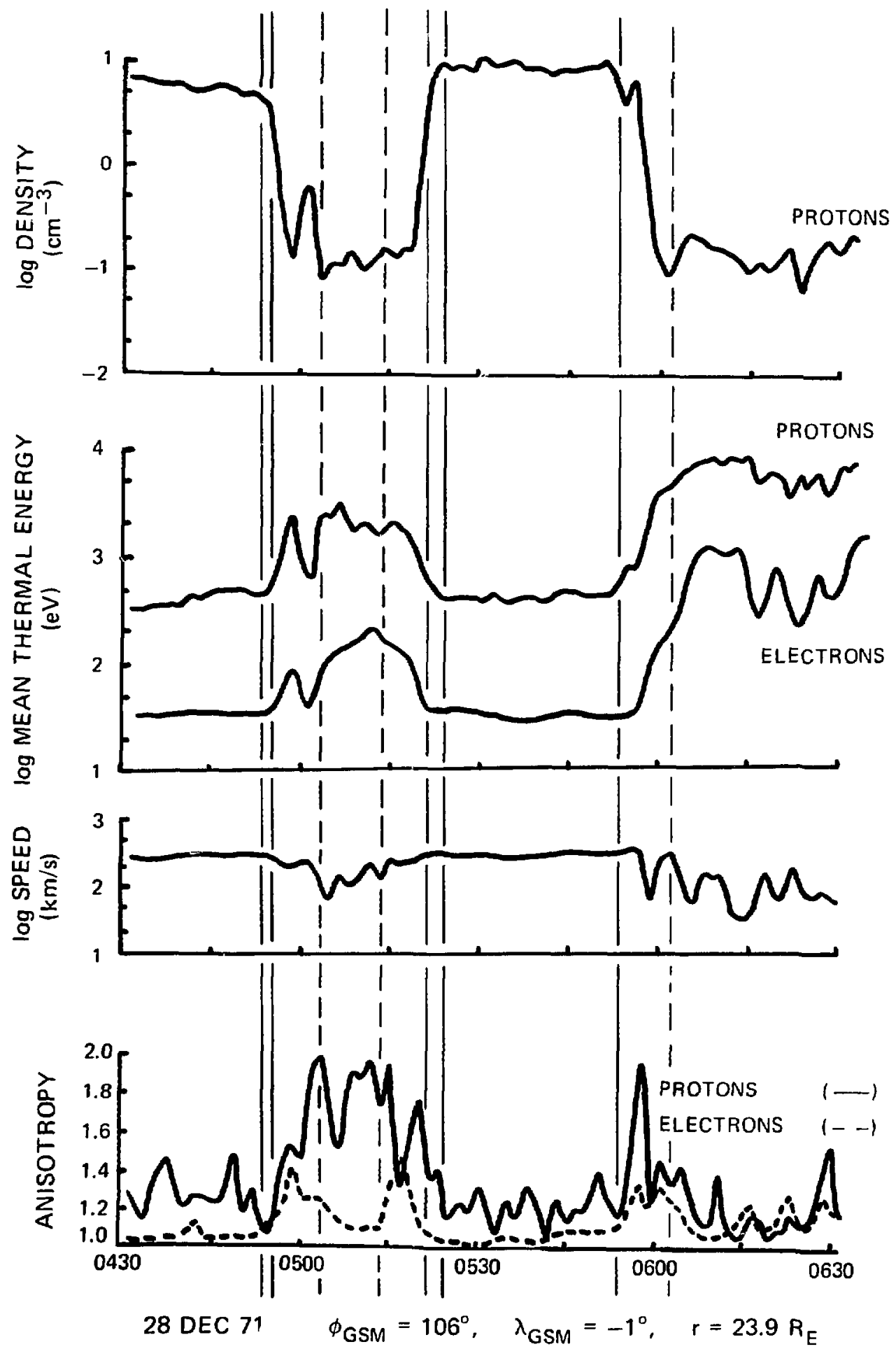




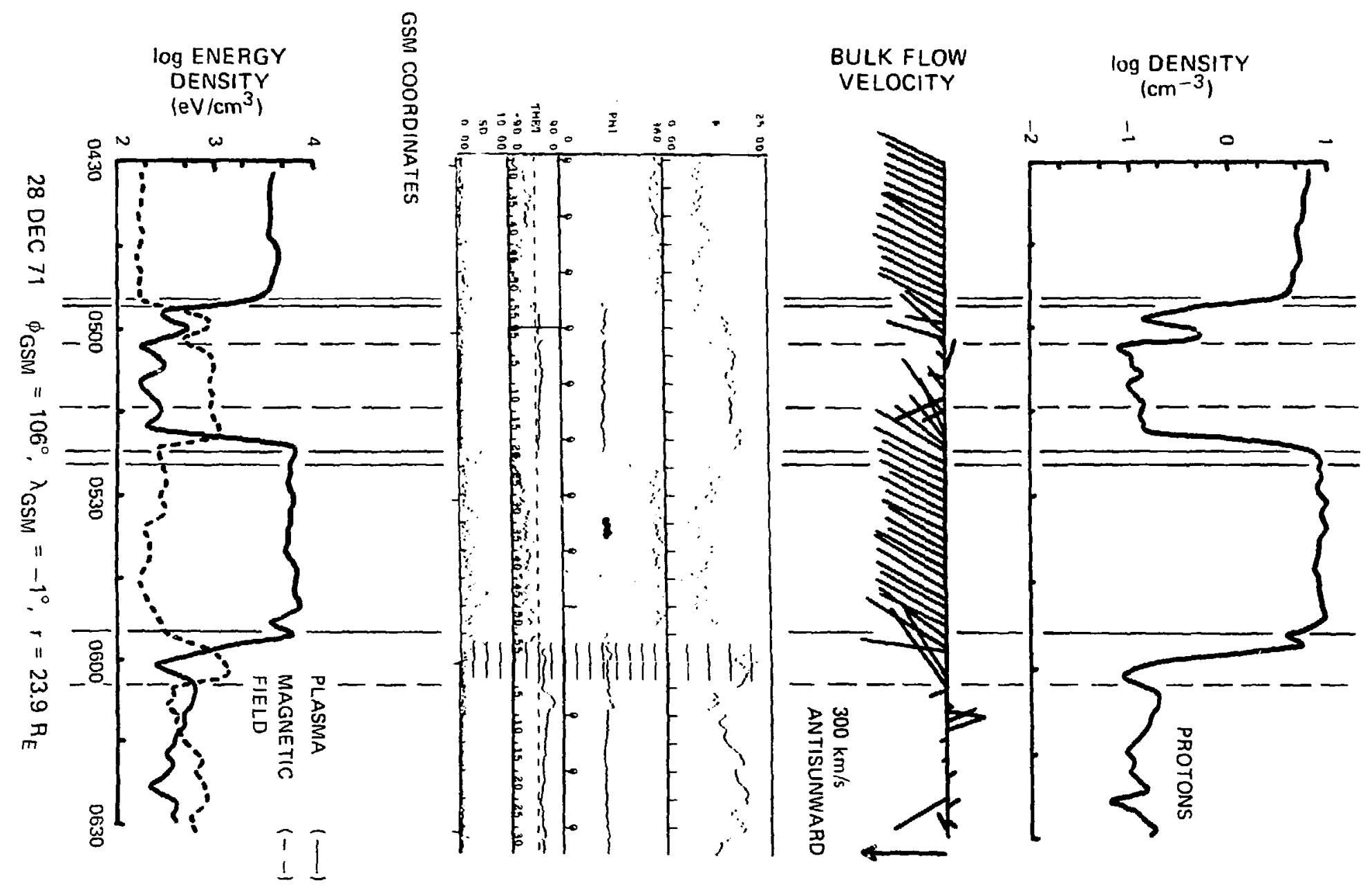




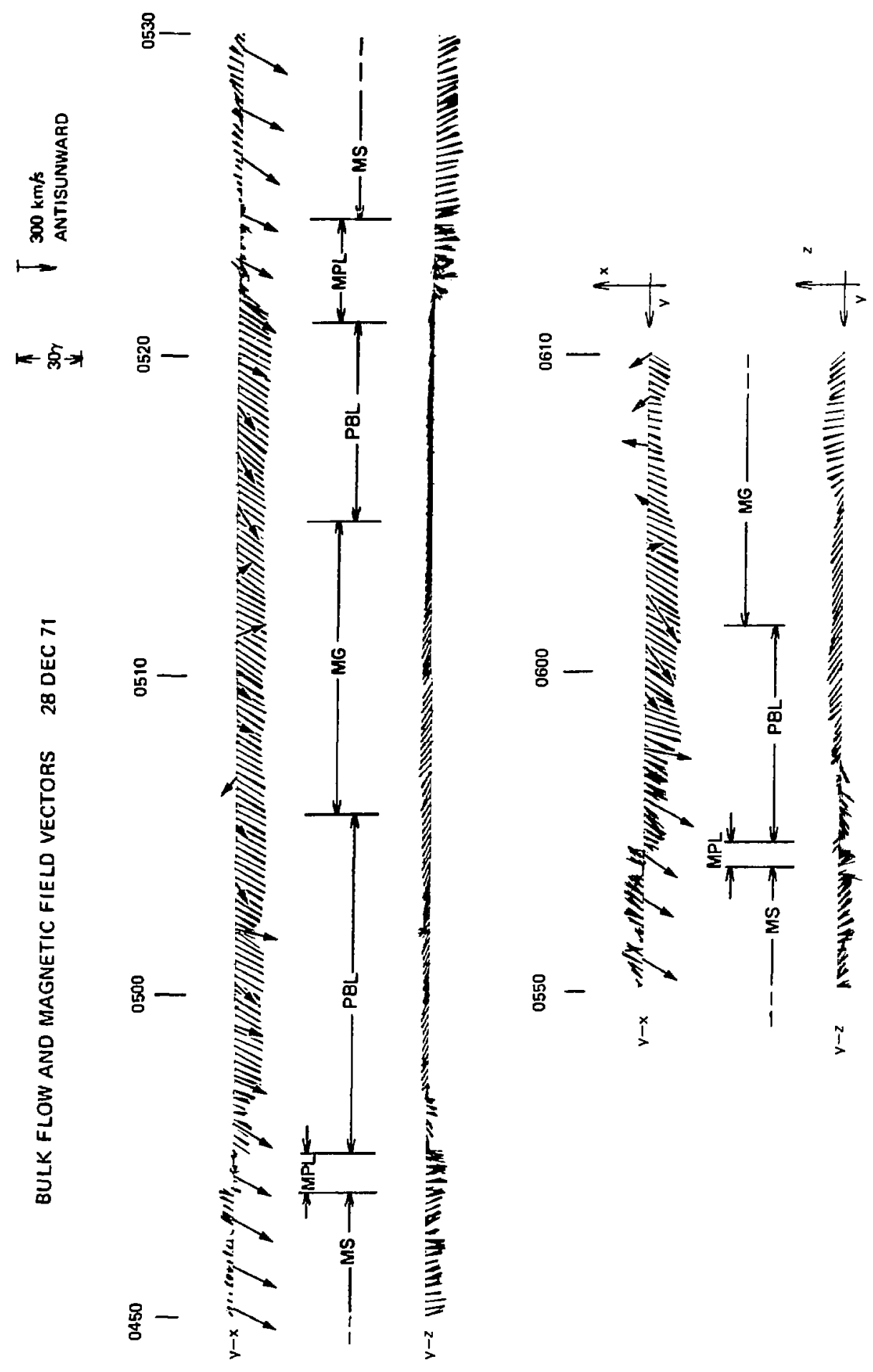


5 January 1972 IMP 6 Crossing

This dusk-side crossing near the equator shows a "detached" plasma region that persists for more than two hours. At times the field is directed almost perpendicular to the ecliptic plane, while magnetosheath-like antisunward flow is observed. Similar to the 22 November 1971 crossing, anisotropy values are highest in the detached plasma region. This region could be truly detached or could reflect a substantial change in PBL thickness.

Significant cross-field bulk plasma flow, as observed in this crossing, is generally observed in the subsolar region or at low latitudes, especially near the geomagnetic equator. Such cross-field flow is generally associated with tailward draping of the outer magnetospheric field. 


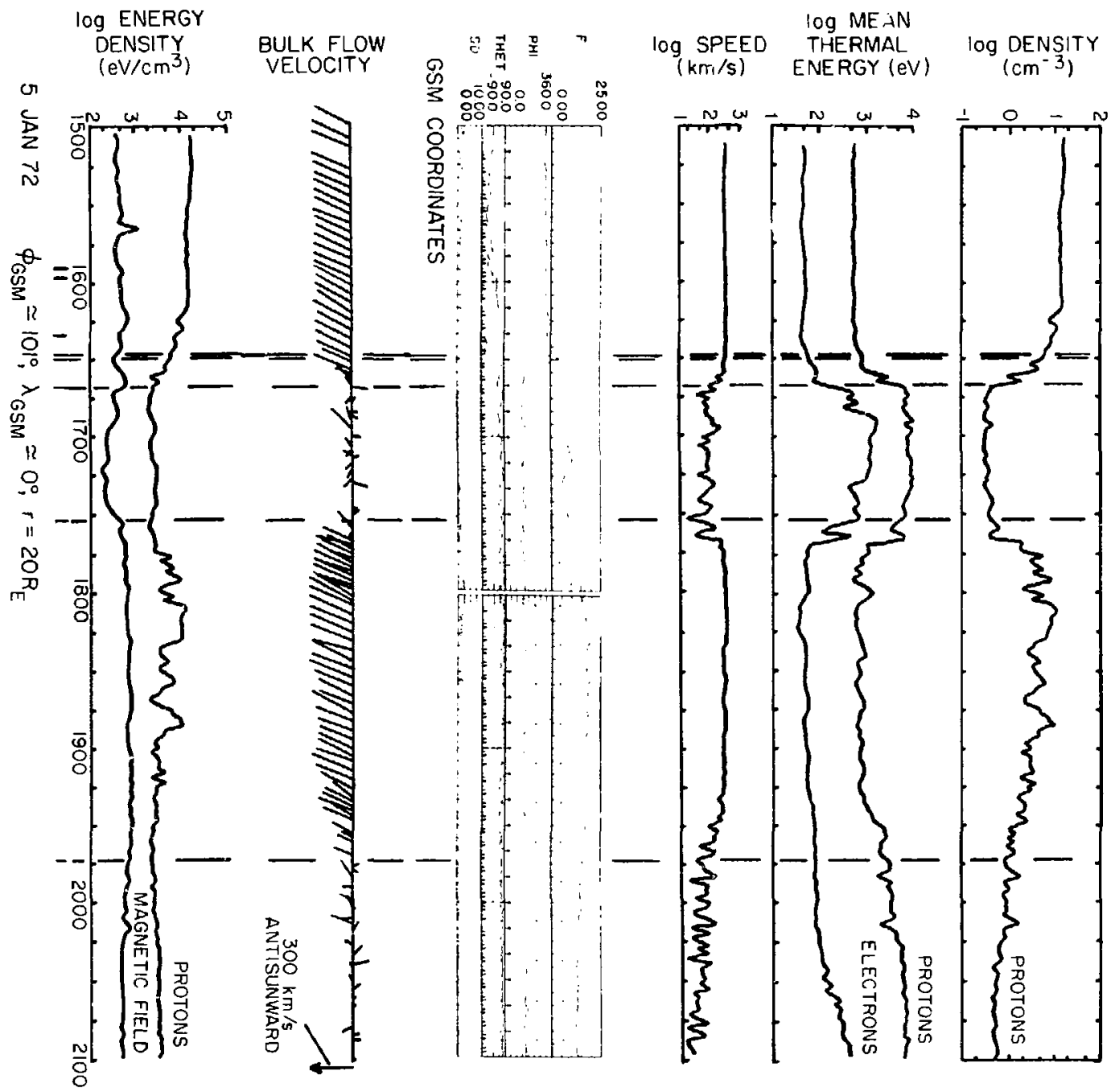


BULK FLOW AND MAGNETIC FIELD VECTORS 5 JAN 72

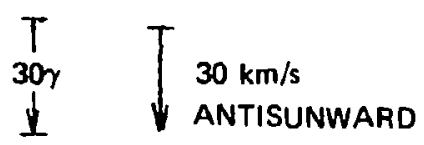

1620

1630

1640

1650

1

1

1

1

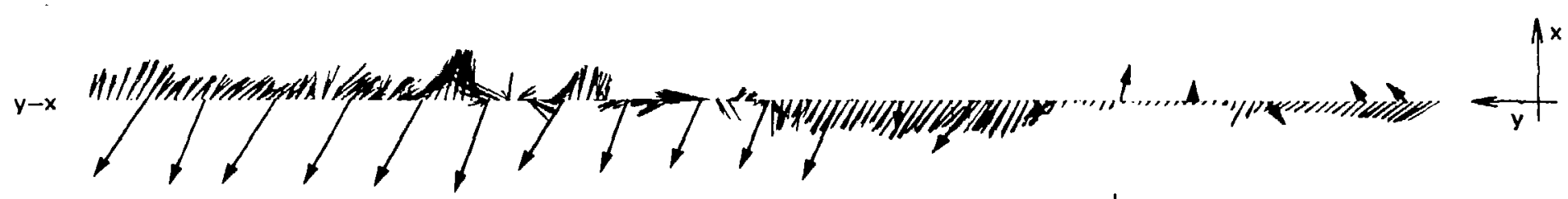

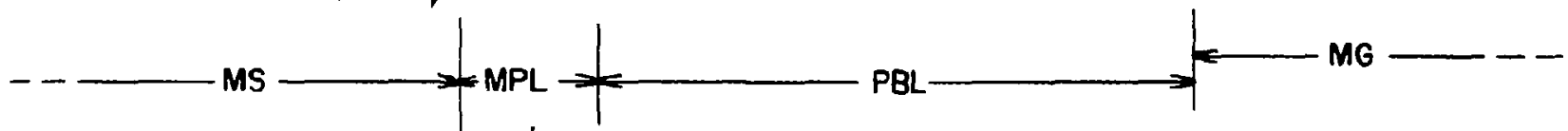

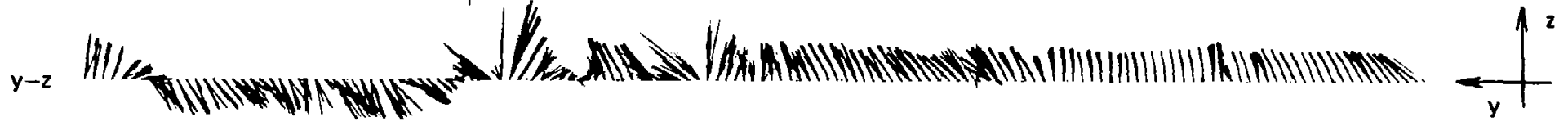


10 January 1972 IMP 6 Crossing

This high-latitude crossing is located on the dusk side of the outer cusp region based on the field direction $\left(\phi_{B}=200^{\circ}, \lambda_{B}=-30^{\circ}\right)$. No clear MPL is evident and the flow field is highly variable and includes sunward component flow consistent with HEOS 2 observations in the cusp region (see Haerendel, 1978). This crossing is also discussed by Falrfield and Hones (1978). 

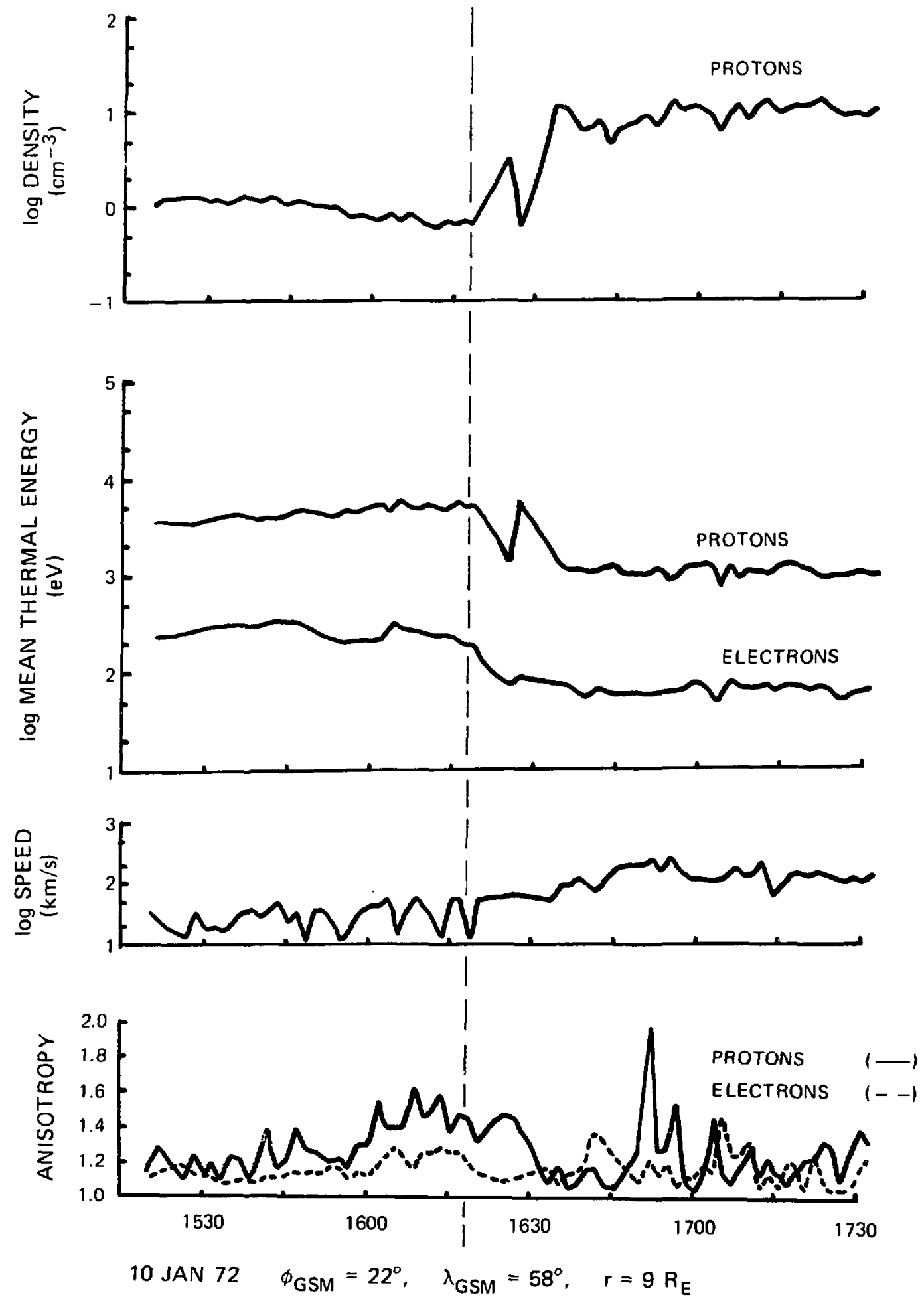


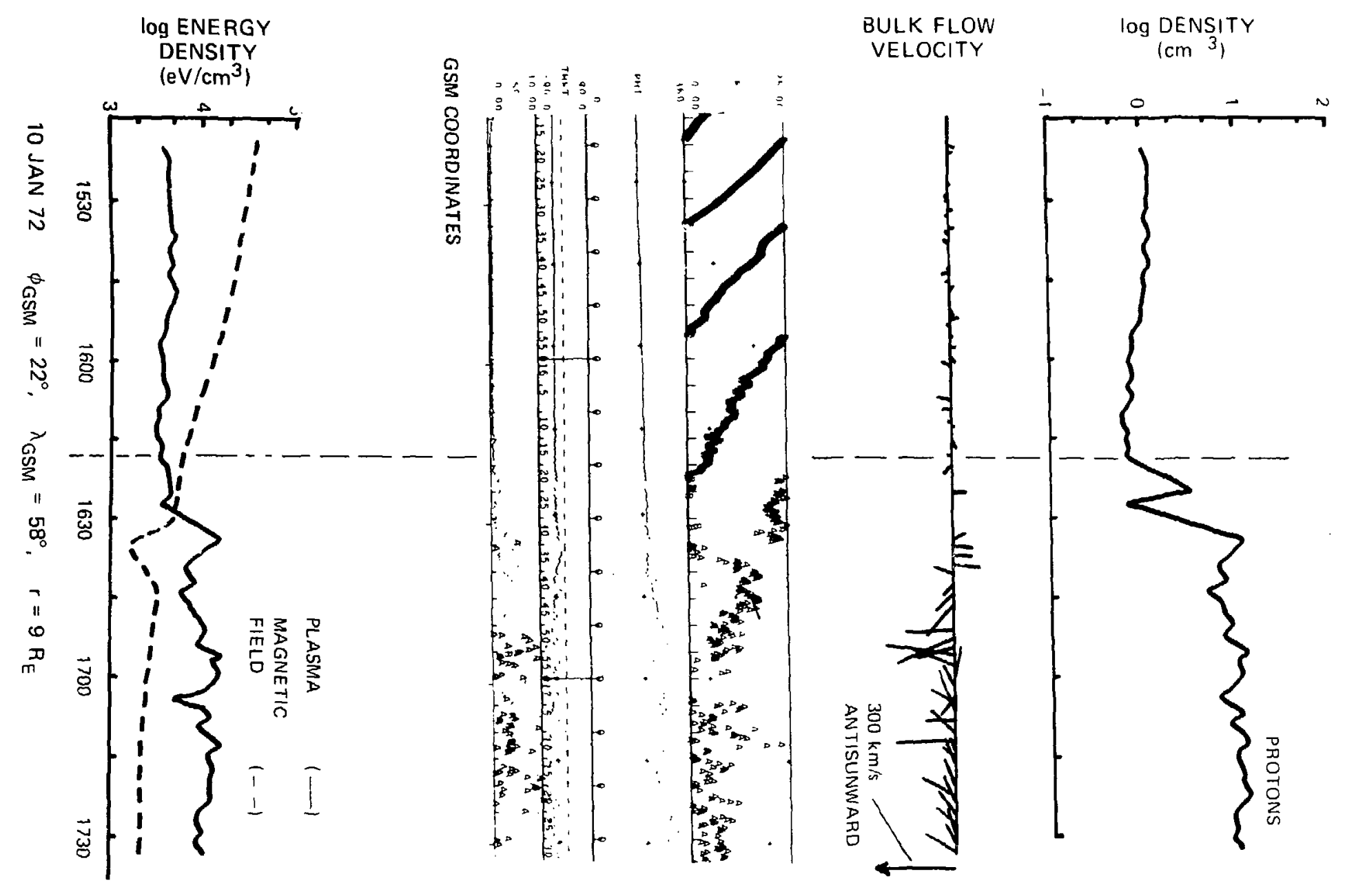




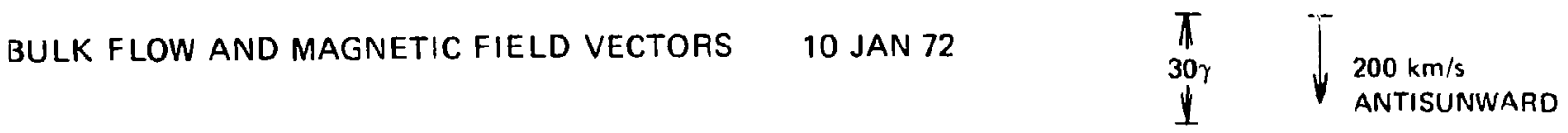

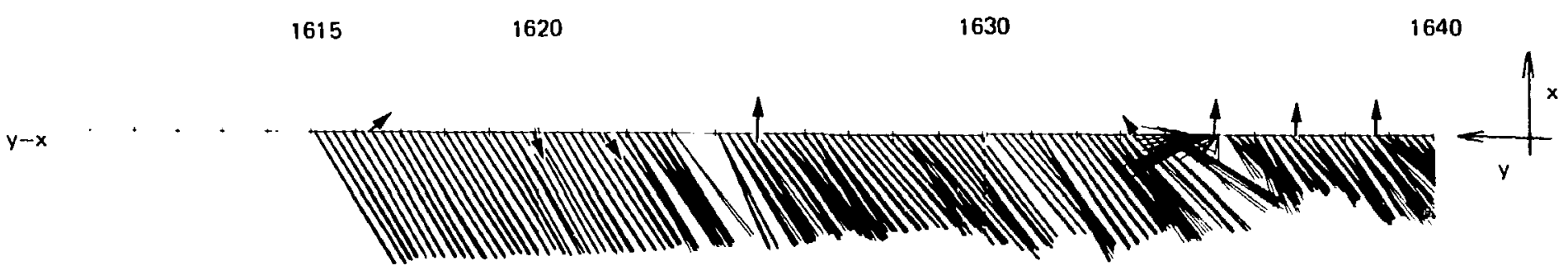

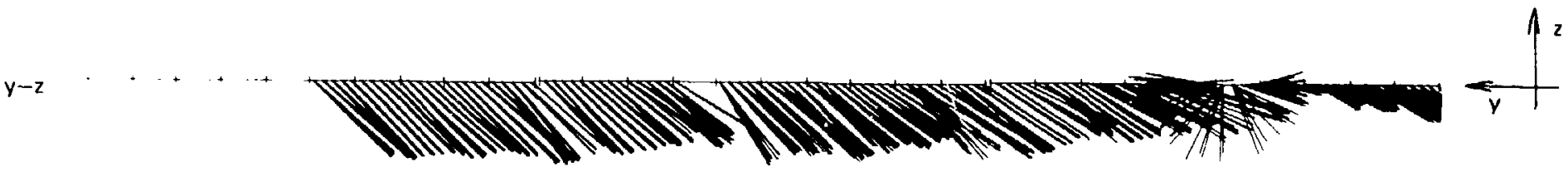


4 February 1972 IMP 6 Crossing

This low-latitude dusk-side crossing shows a field rotation in $\phi_{B}$ at 0052 UT earthward of a clear change in $\lambda_{B}$ at $n 0044$ UT. In either case, the PBL is clear with significant cross field flow and tailward field draping. Bulk plasma flow in the PBL has a larger angle with respect to the $\mathrm{X}_{\mathrm{GSM}}$-axis than the nearby magnetosheath flow. This shift in bulk plasma flow is a common feature of the PBL as shown by most of the examples presented in this thesis. Based on observations from a single satellite it is difficult to determine whether this directional shift corresponds to a net component of flow normal to the magnetopause. It. can be caused partly by a shift in flow direction in the plane tangent to the magnetopause which is then effectively projected onto the ecliptic plane by the $\pm 45^{\circ}$ sampling aperture of the electrostatic analyzer (see Appendix A). 


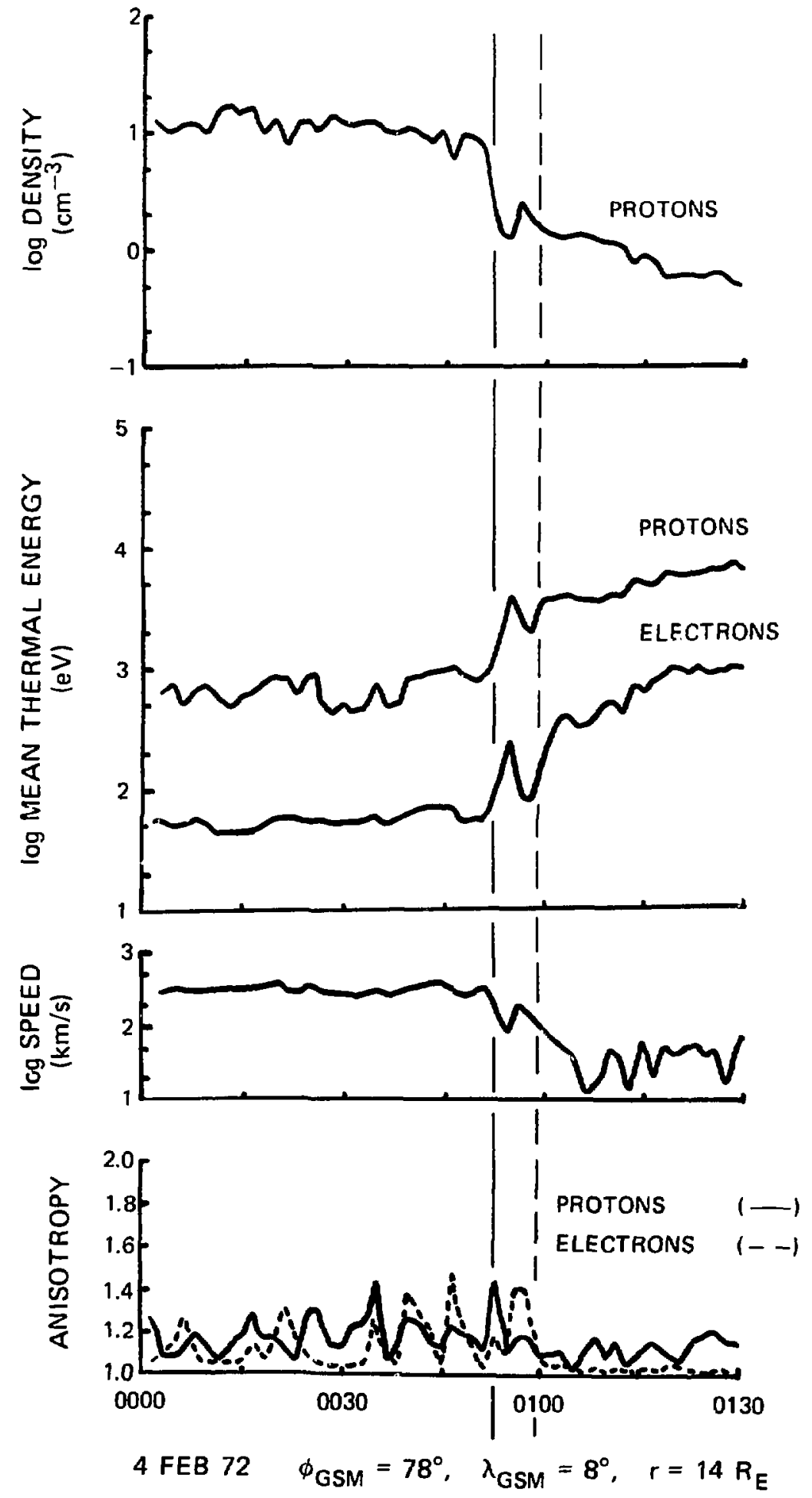



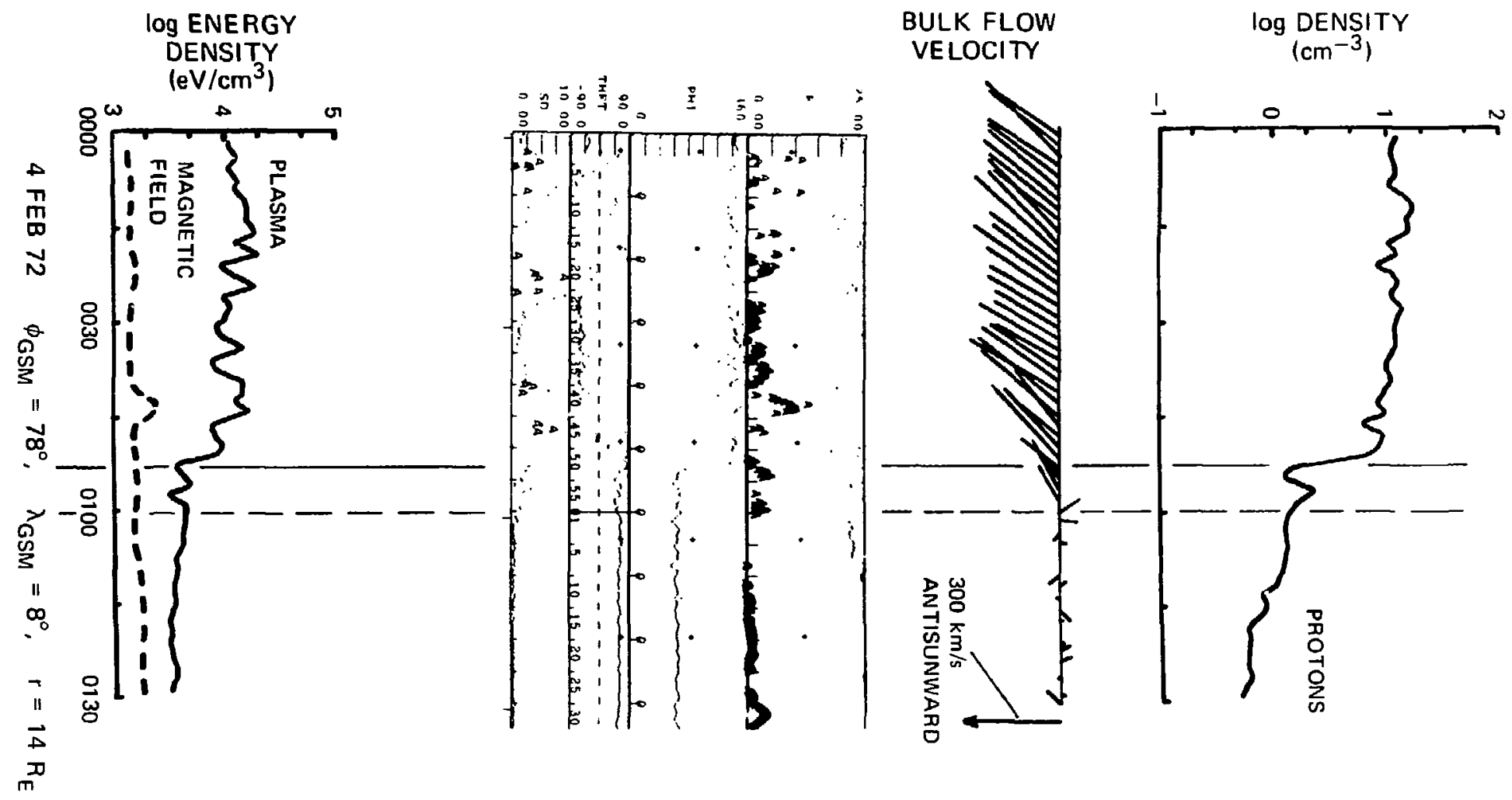


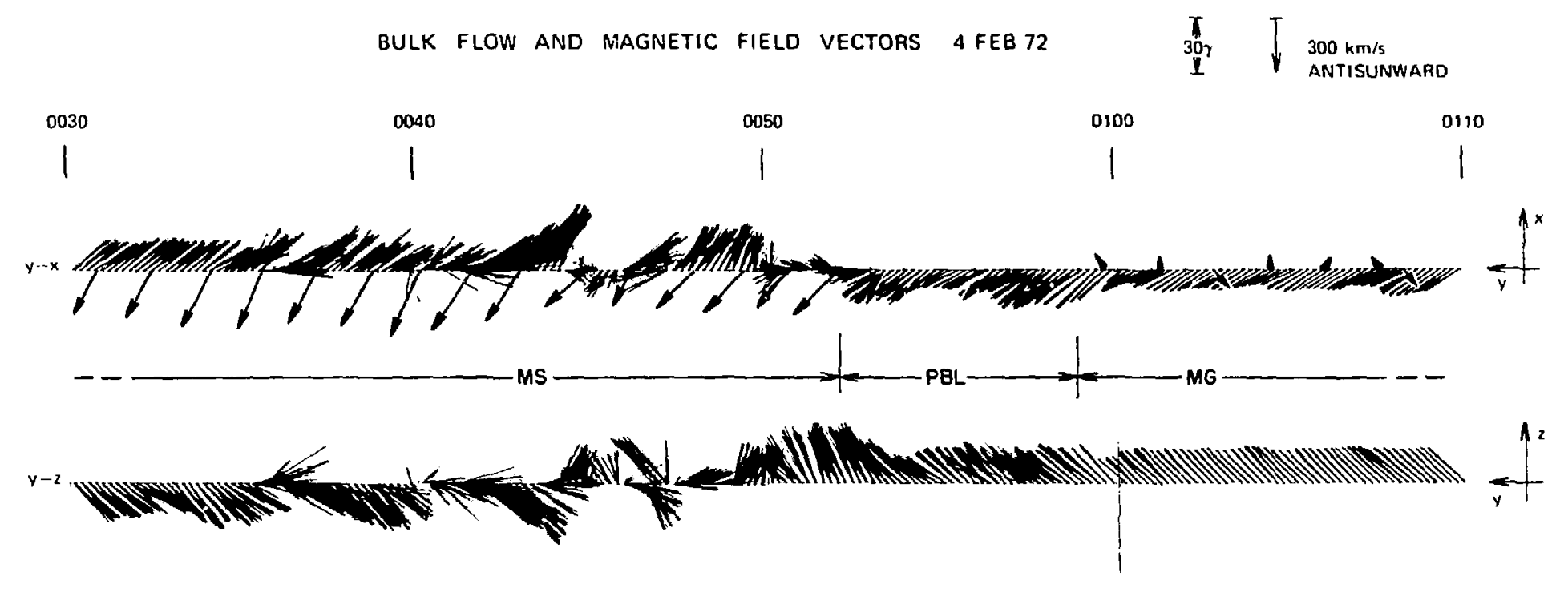


28 February 1972 IMP 6 crossing

This low-latitude dusk-side crossing is highly variable in both plasma and field characteristics although the MPL and PBL locations are clear. Significant cross-field flow as well as tailward field draping is present. Highly variable PBL signatures are frequently observed and reflect the presence of substantial space and/or time variations in the MPL-PBL region. One high-density high-field-magnitude region is located at $\backsim 2236 \mathrm{UT}$. It has a magnetospheric direction for $\lambda_{B}$ and otherwise has magnetosheath-like characteristics. 


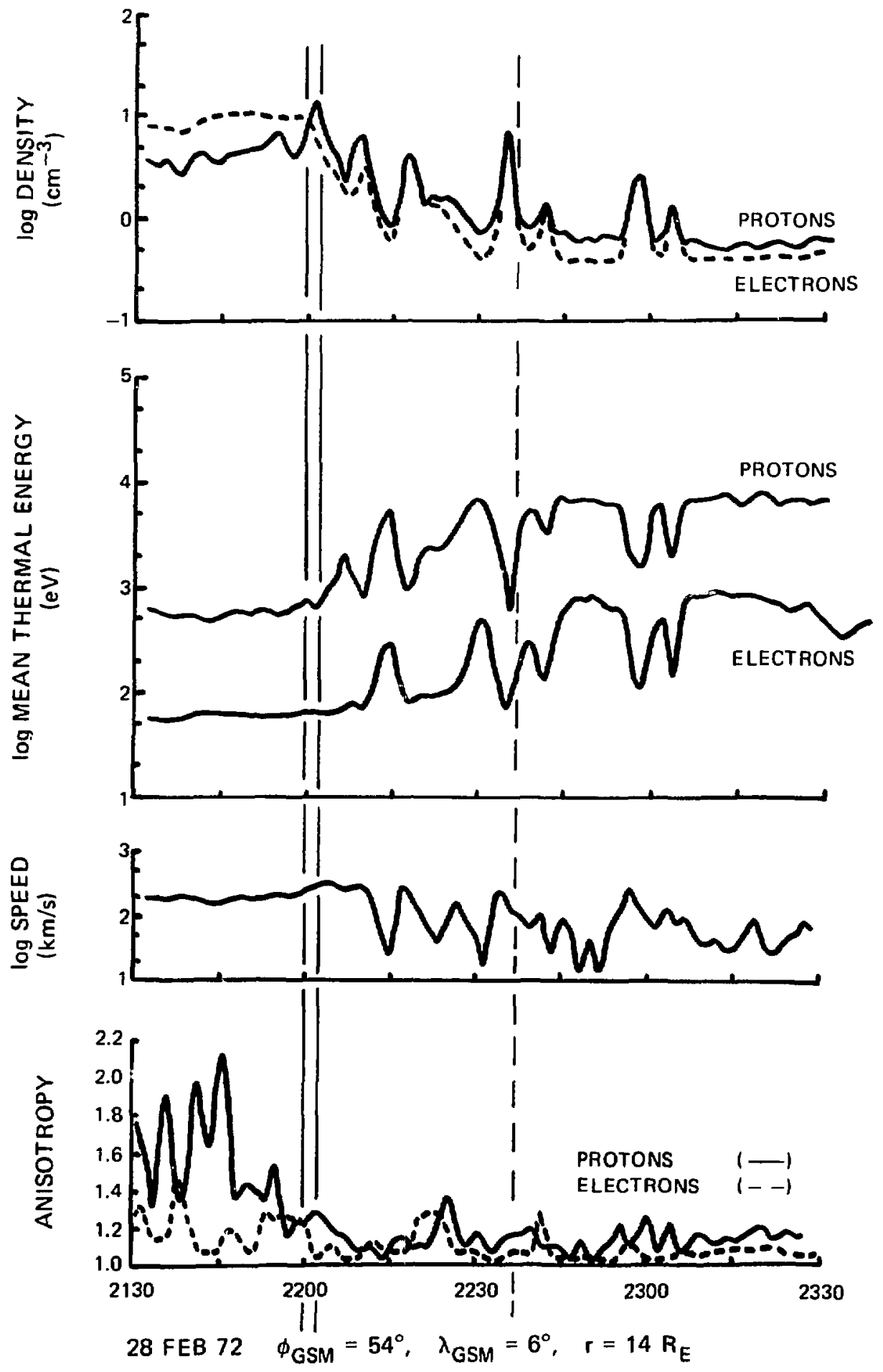



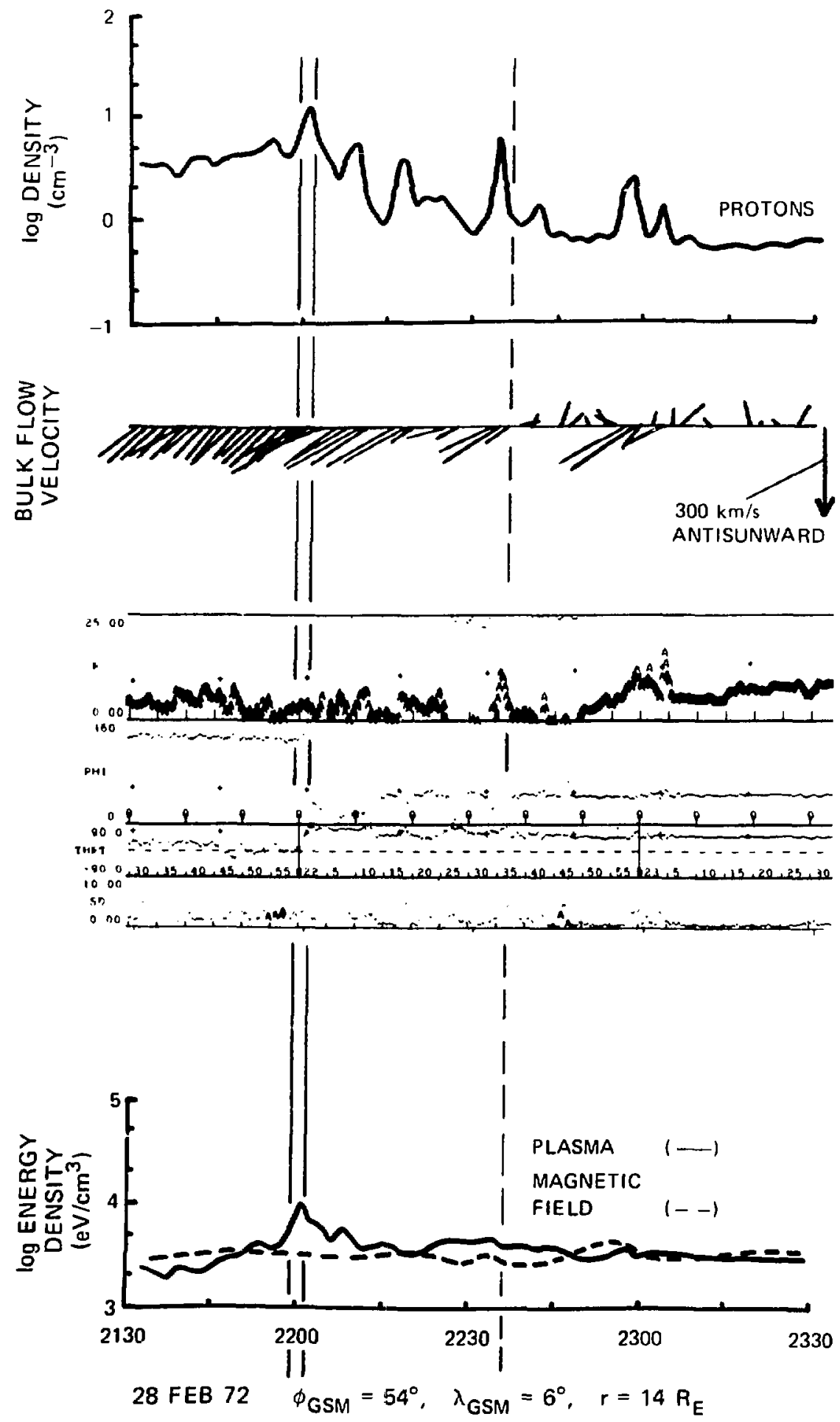


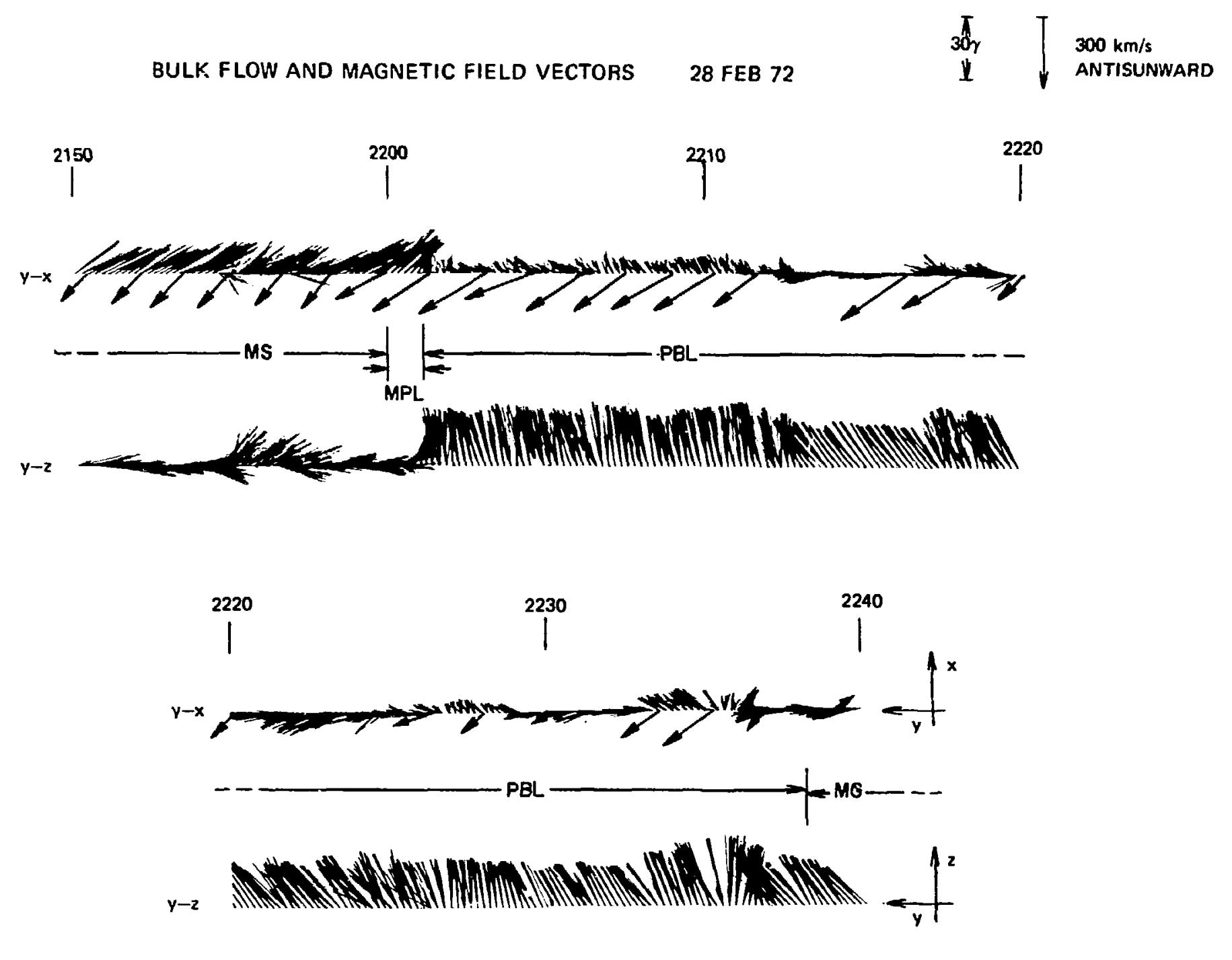


8 March 1972 IMP 6 Crossing

This low-latitude dusk-side crossing has no clear MPL. Significant cross-field bulk plasma flow is present along with tailward field draping. Ion anisotropy is large in the nearby magnetosheath and is associated with substantial density depletion (Crooker et al., 1978). Identification of the PBL is this case depends on a comparison of all plasma and field parameters as shown. IMP 6 crossings on 7 July 1971 and 16 June 1973 (section 2.3.4) al so show no clear MPL. 


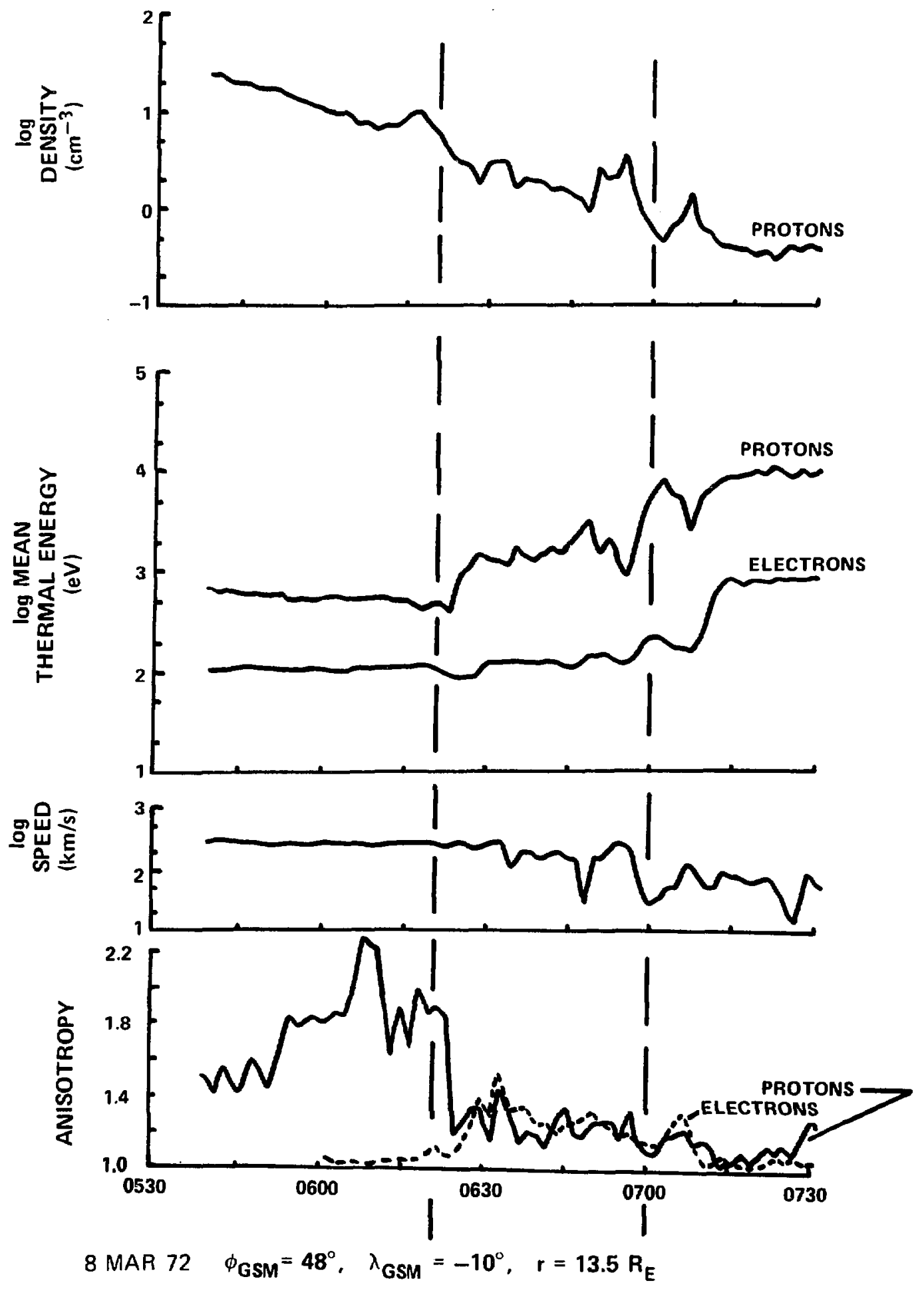




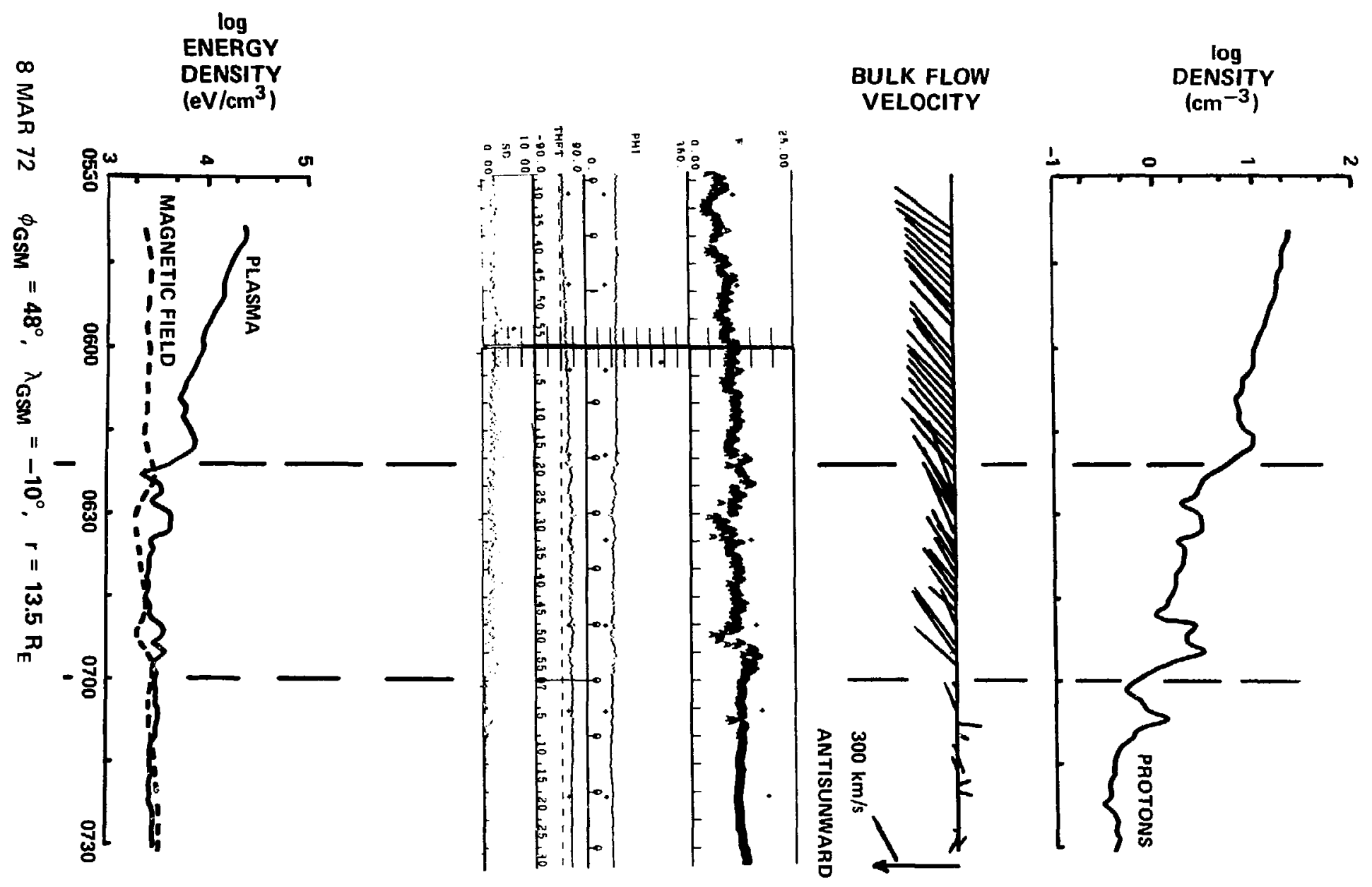




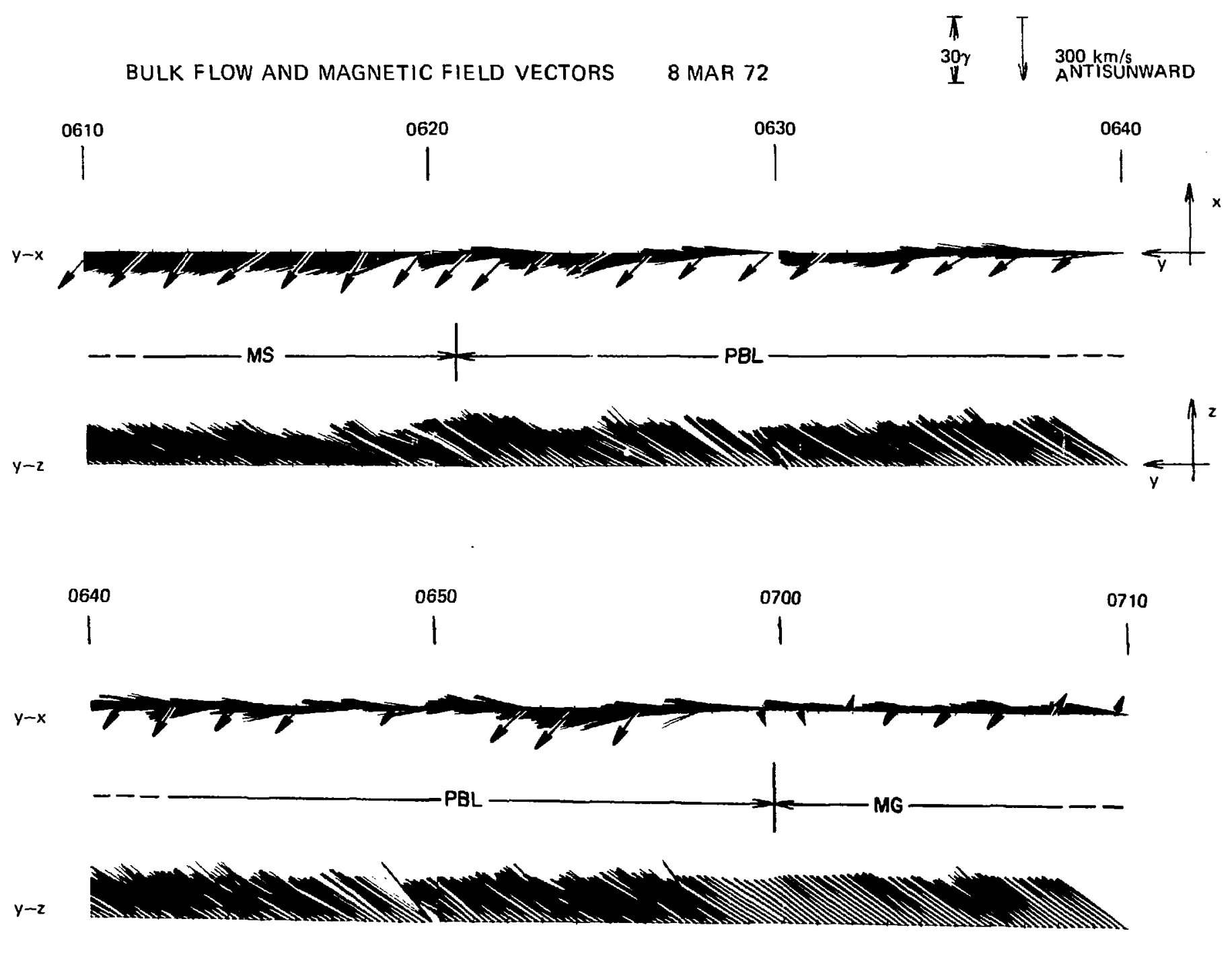


24 Karch 1972 IMP 6 Crossing

This low-latitude dusk-side crossing shows a clear shift in $\lambda_{B}$ at $\$ 2151$ UT with an added rotation occurring later at $\$ 2205$ UT, similar to the 4 February 1972 crossing. Plasma parameters are at magnetosheath levels through the MPL and up to near the $\phi_{B}$ transition. The field magnitude has a gradient scale length comparable to the PBL thickness. This is a common feature and supports the PBL identification given for this case.

Plasma flow in the nearby magnetosphere is often difficult to measure because of low densities and poor counting statistics. For this crossing, however, there appears to be a significant net sunward component of bulk flow, which is consistent with the convective flow pattern envisioned by Axford and Hines (1961) (section 3.2.1). 


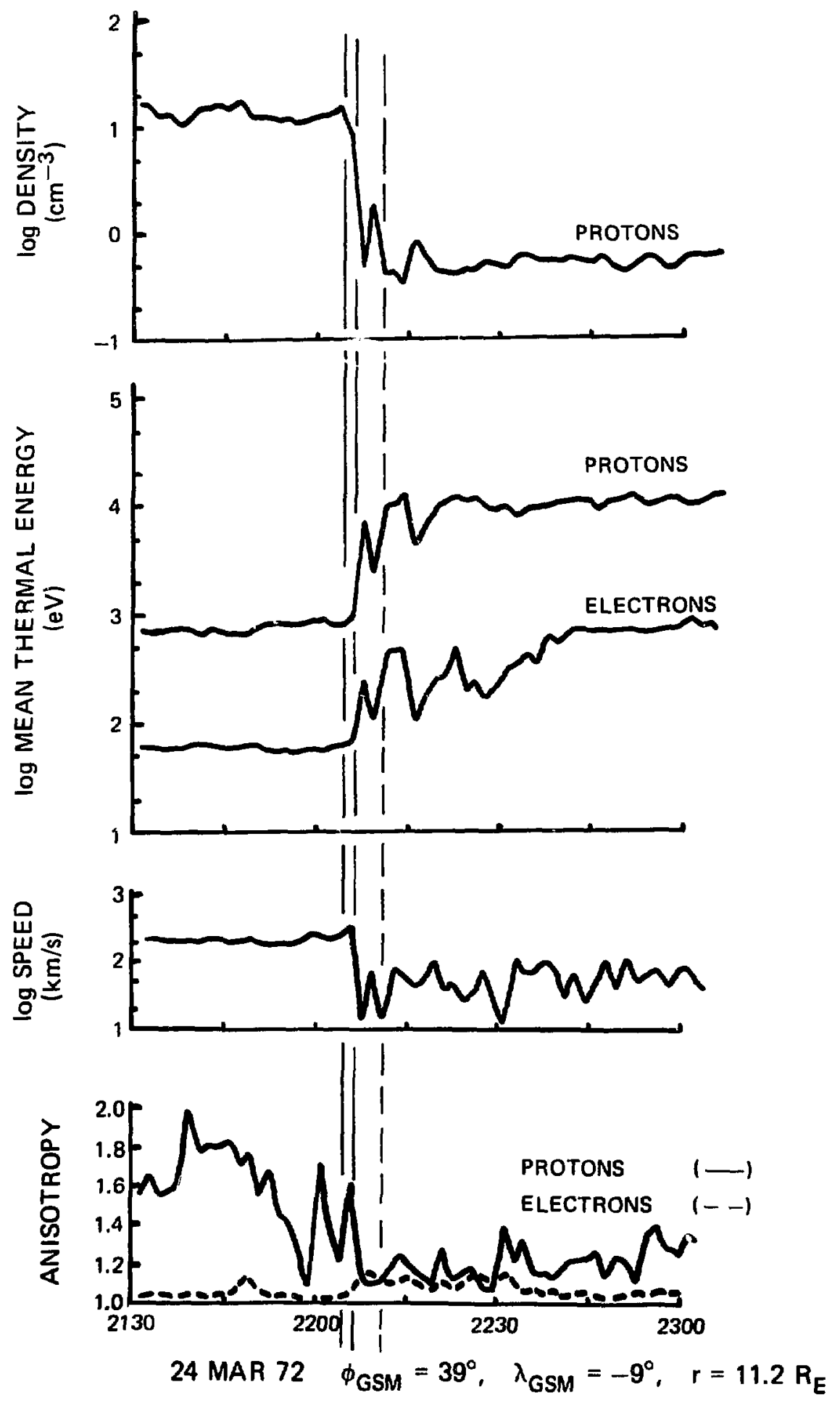




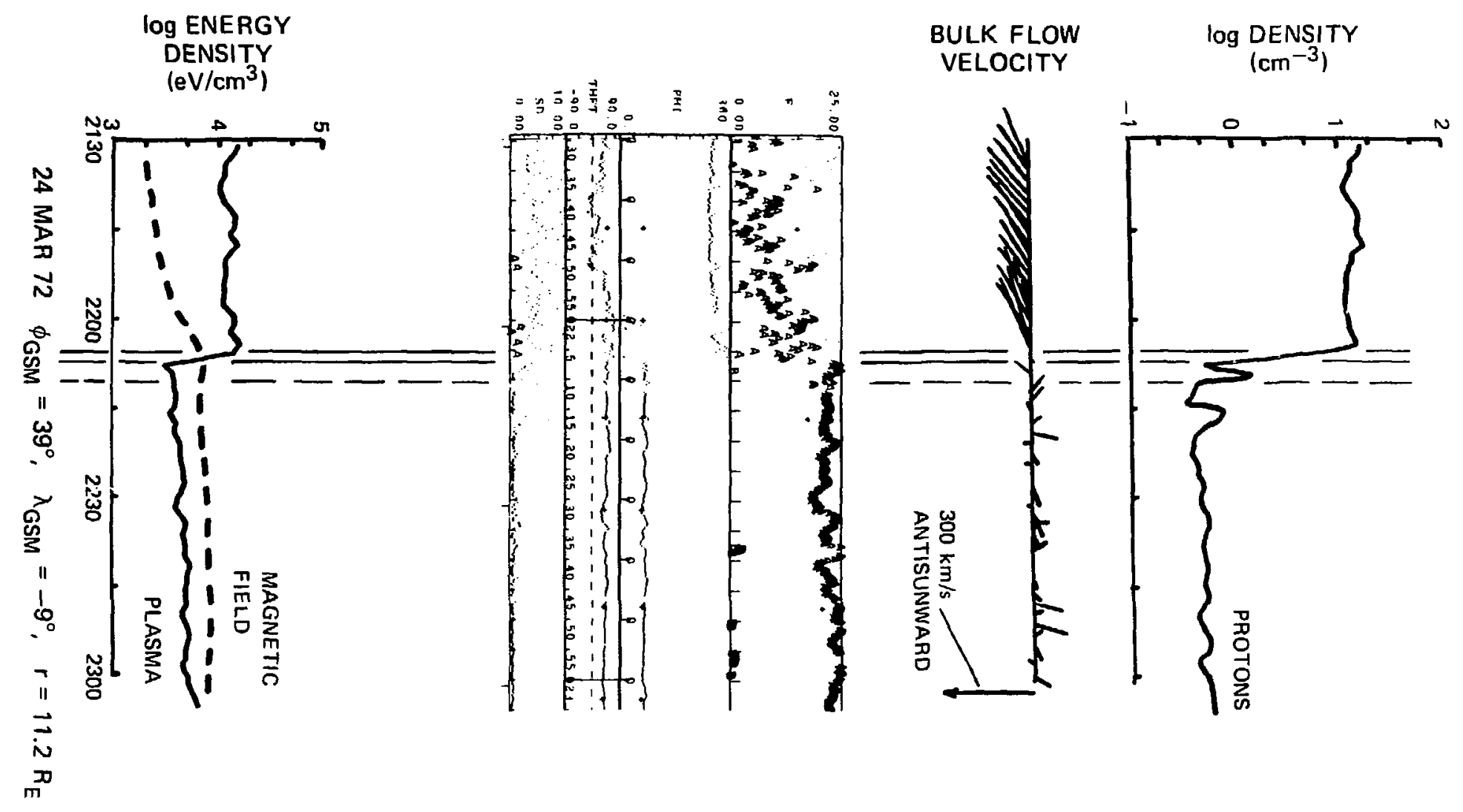


BULK FLOW AND MAGNETIC FIELD VECTORS 24 MAR $72 \quad \begin{gathered}\pi \\ 30 \gamma \\ \mathbf{L}\end{gathered} \quad\left[\begin{array}{l}300 \mathrm{~km} / \mathrm{s} \\ \text { ANTISUNWARD }\end{array}\right.$

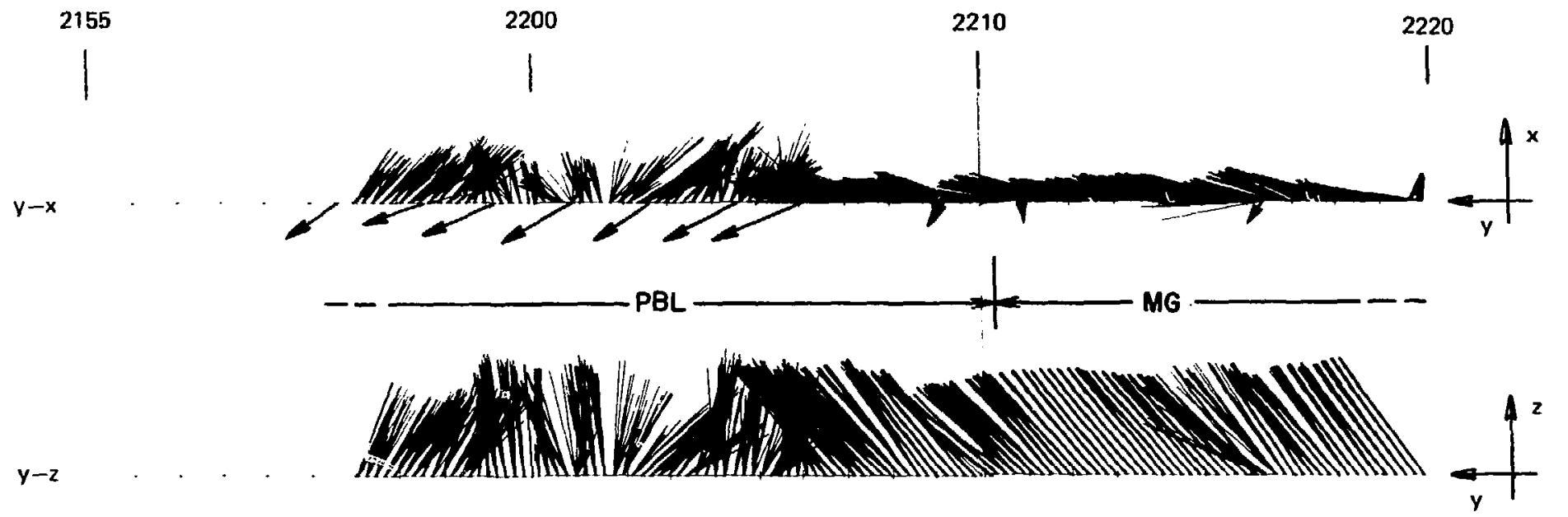


29 March 1972 IMP 6 Crossing

This high-latitude crossing is located on the dawn side of the outer Busp region. Similar to the 16 December 1971 and 10 January 1972 crossings, the flow field is highly variable in the cusp region PBL (called "entry layer" by Haerendel et al., 1978). The MPL is not well defined since $\phi_{B}$ returns to its magnetospheric values after $1400 \mathrm{UT}$. Enhanced field fluctuations appear near the MPL and large ion anisotropy values occur in the PBL. Some of the plasma flow in the inner part of the $\mathrm{PBL}$ is consistent with flow into the cusp, whereas plasma flow during the anisotropy peak could be directed roughly antisunward and tangent to the magnetopause. Significant cross-field bulk plasma flow is present throughout this crossing except for the isolated sunward component flow seen at $\backsim 1328 \mathrm{UT}$.

Energetic electron (47- to 350- keV) pitch-angle distributions for this crossing show an isotroplc distribution in the nearby magnetosheath and a pancake-shaped distribution in the PBL. Combined with the magnetospheric orientation of $\mathrm{PBL}$ field lines, these observations indicate that the PBL is located on closed field lines. 


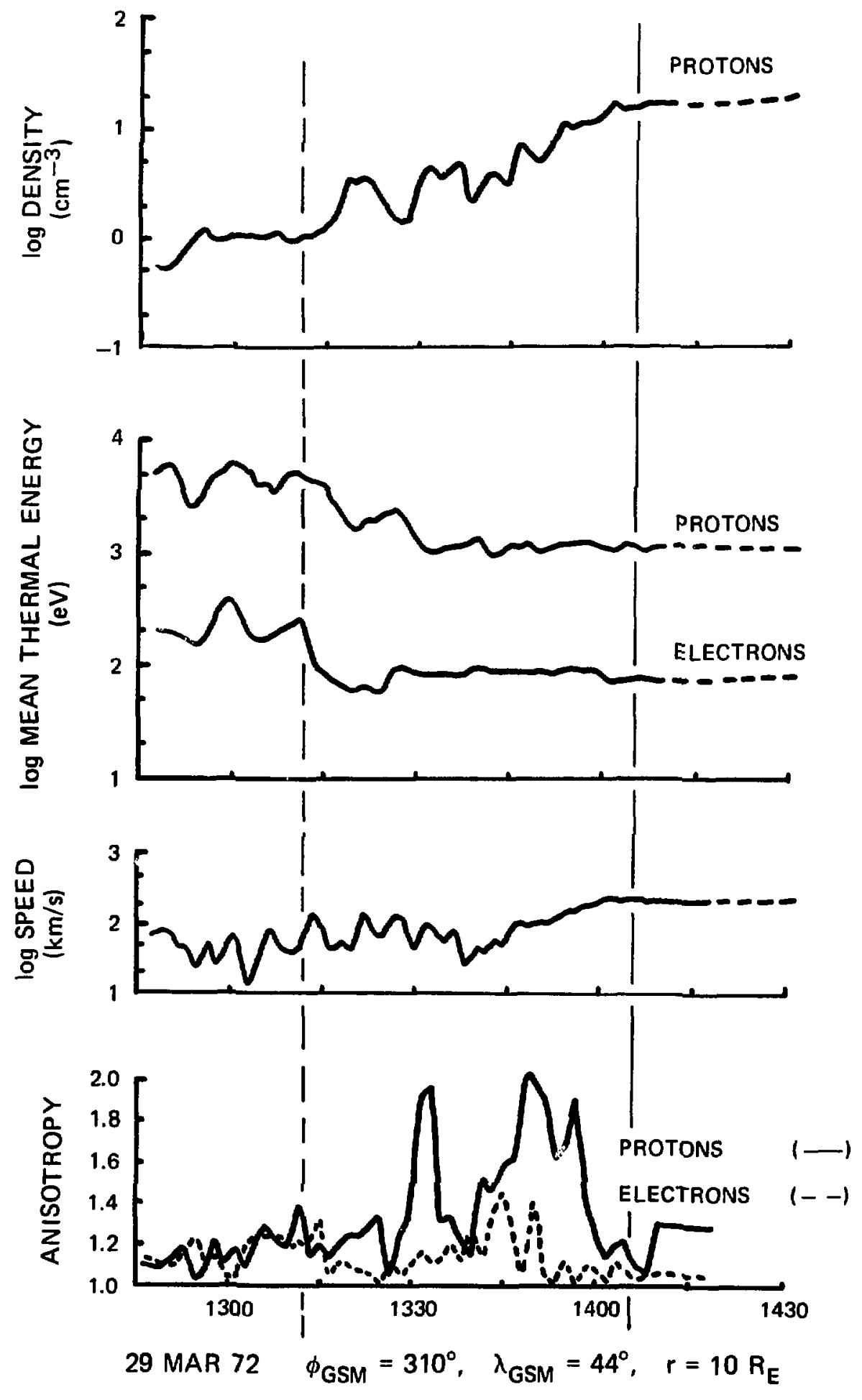




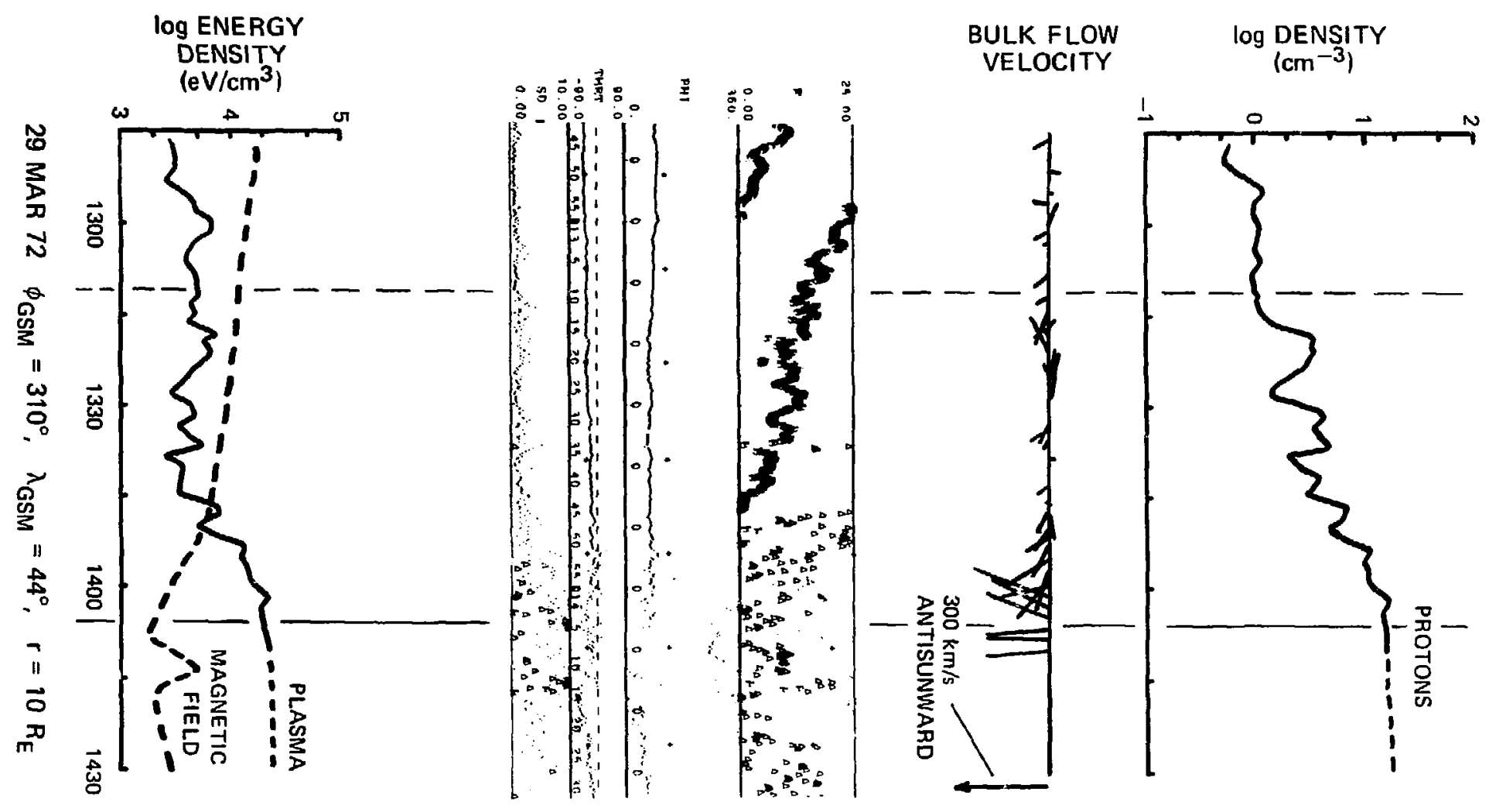


BULK FLOW AND MAGNETIC FIELD VECTORS

29 MAR 72

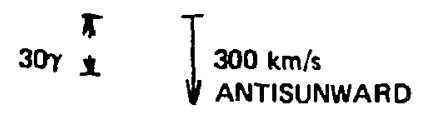

1330
1340
1310

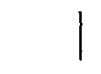

1320

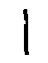

1
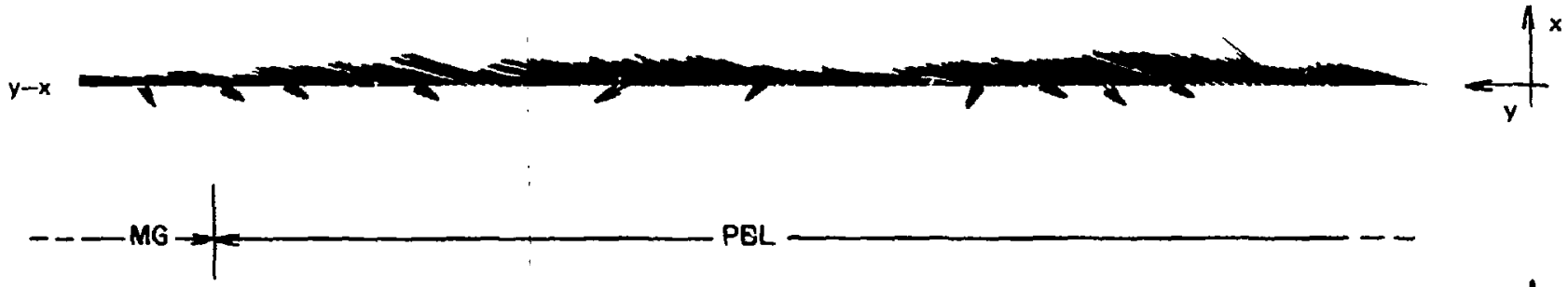

$y-2$

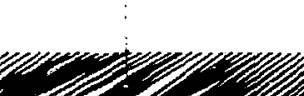

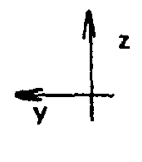

1340

1350

$y-x$

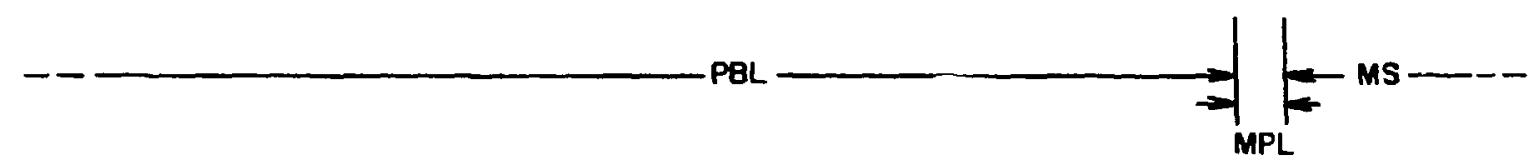

$y-2$

$\stackrel{\infty}{\infty}$

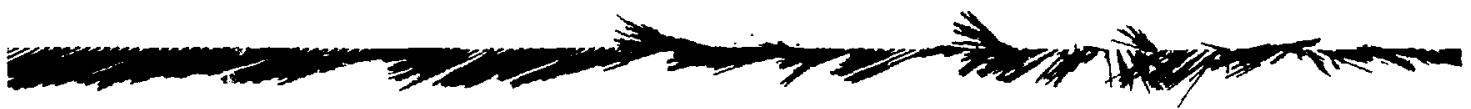


ENERGETIC ELECTRON (47- to 350-keV)

PITCH-ANGLE DISTRIBUTIONS, 29 MARCH 1972

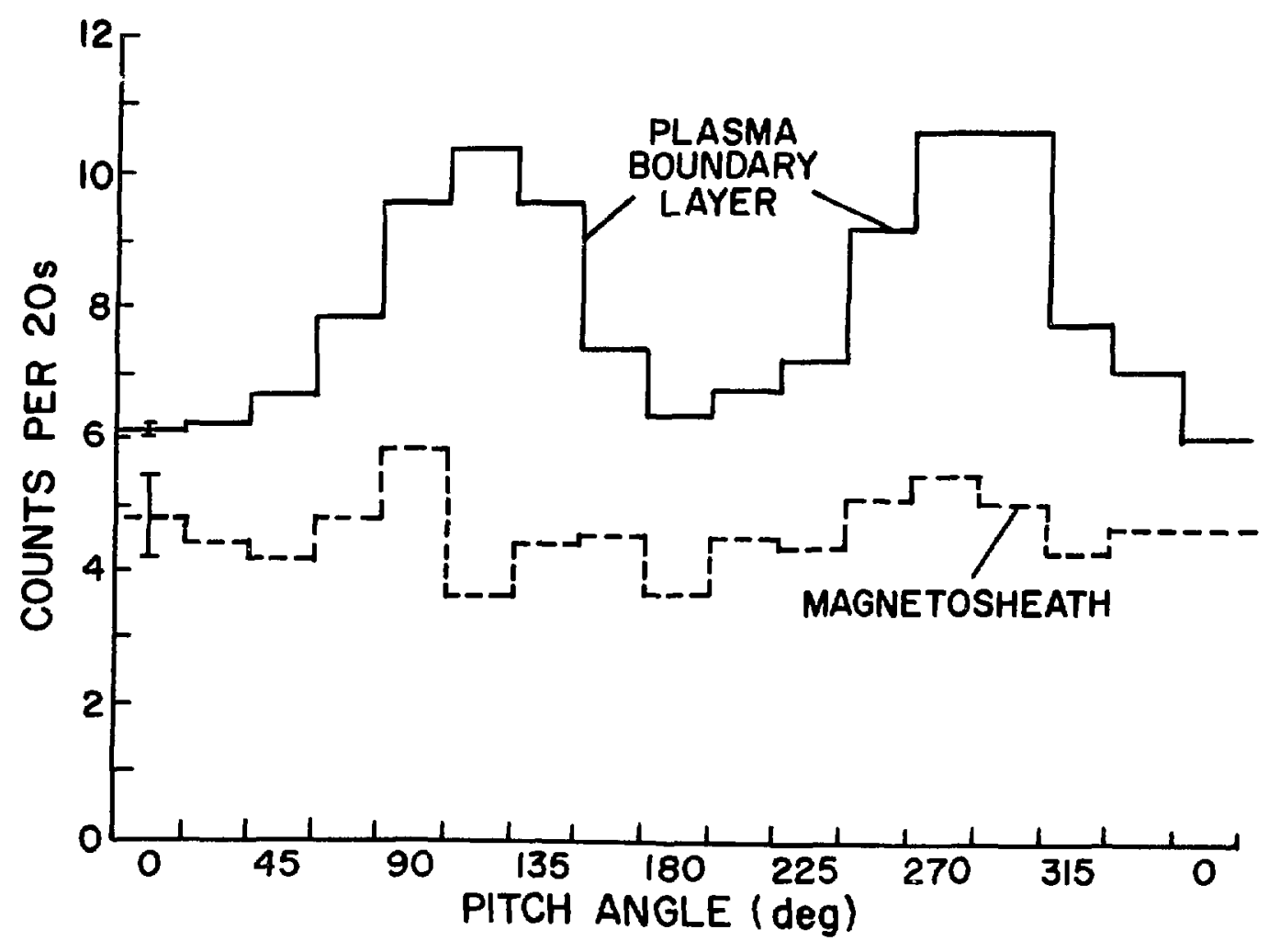


1 May 1972 IMP 6 Crossing

This low-latitude dusk-side crossing has distinct changes in $\lambda_{B}$ at s0821 UT with a $\phi_{B}$ rotation located farther earthward (c.f., 4 February 1972 and 24 March 1972). Most of the PBL is distinctly magnetosheathlike with substantial cross-field flow components and tailward field draping. an isolated region of antisunward flow appears at 0909 UT. The flow direction suggests that this is not caused bj a simple re-entry of the PBL. 


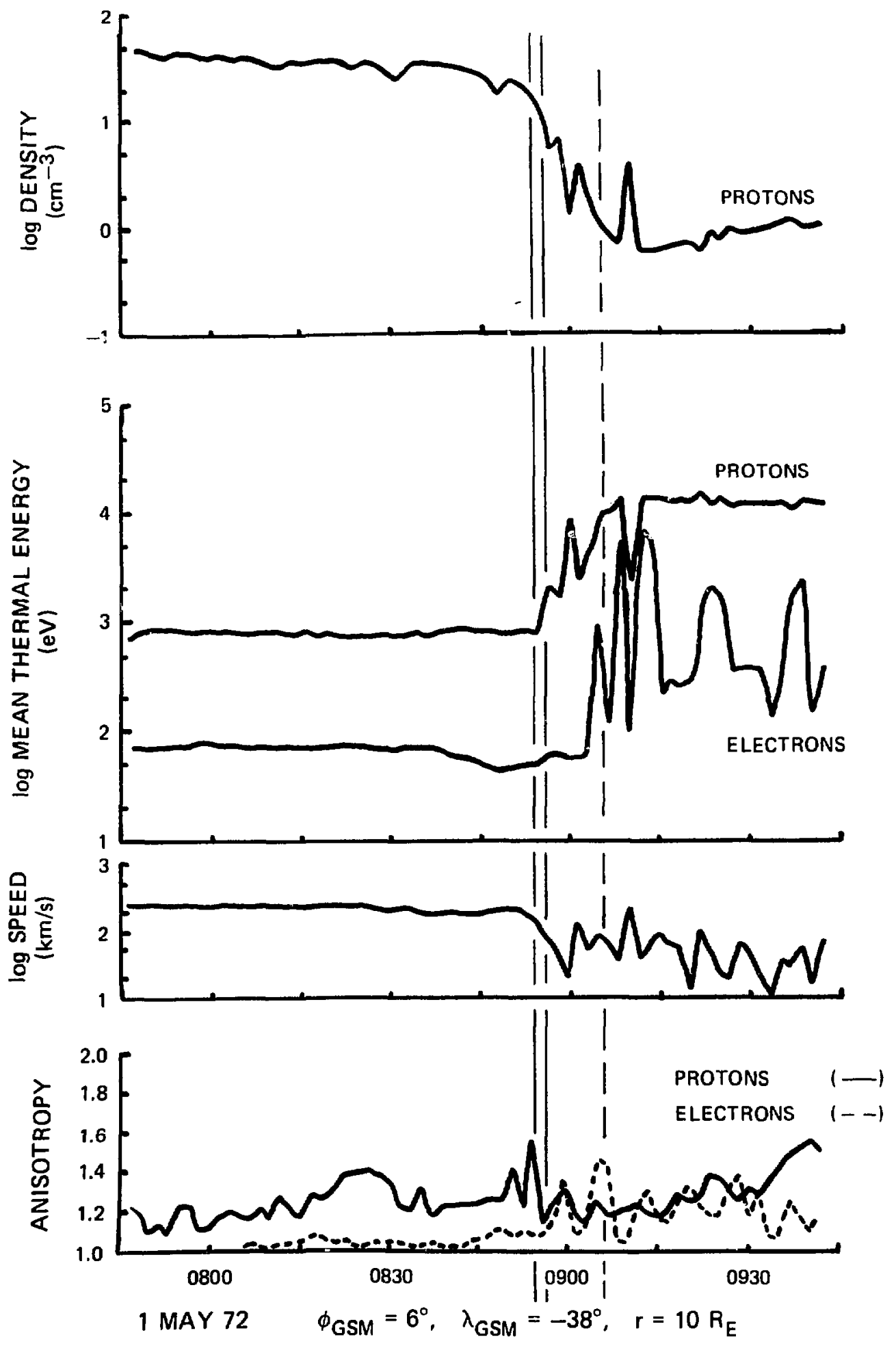



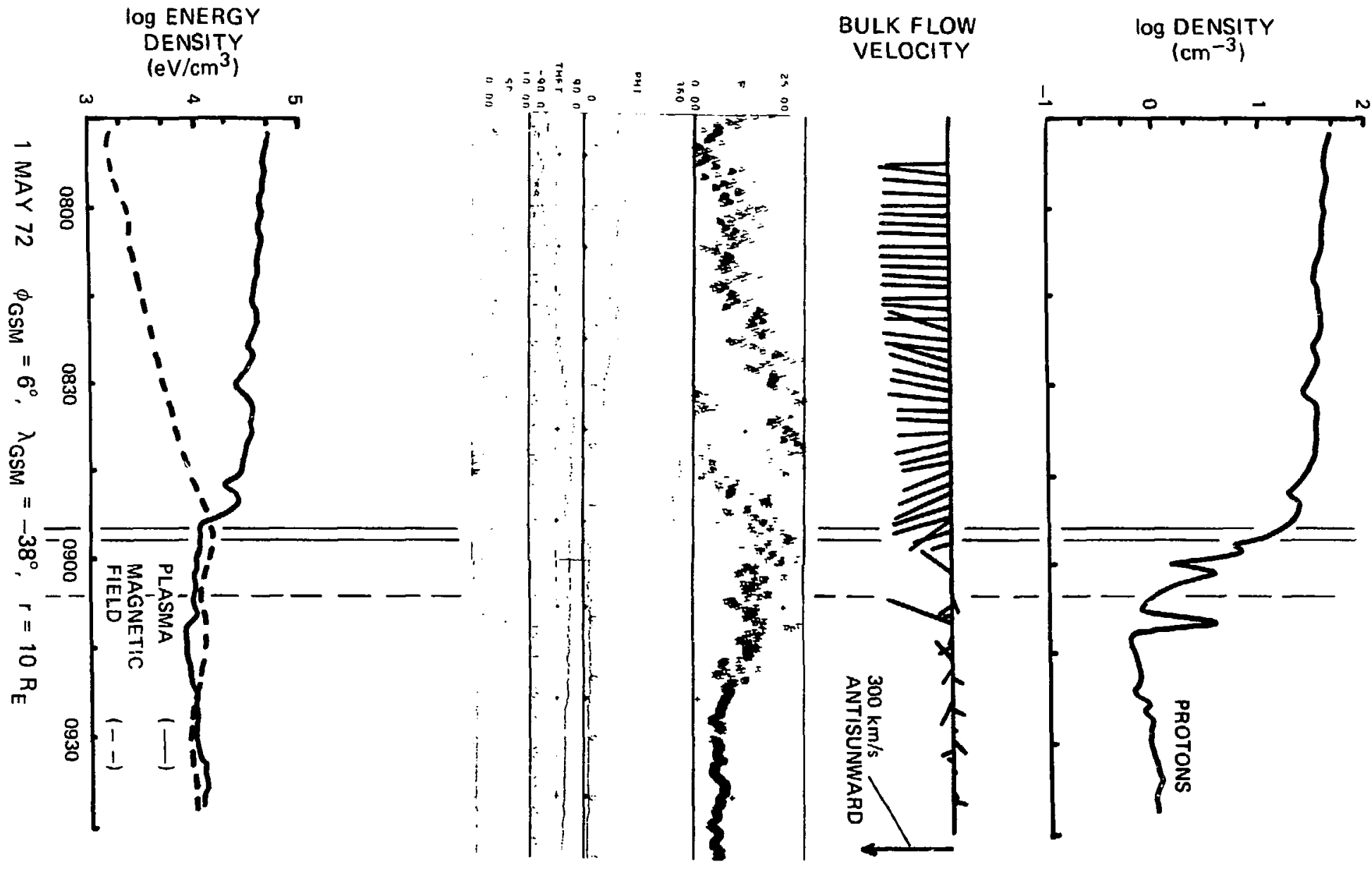


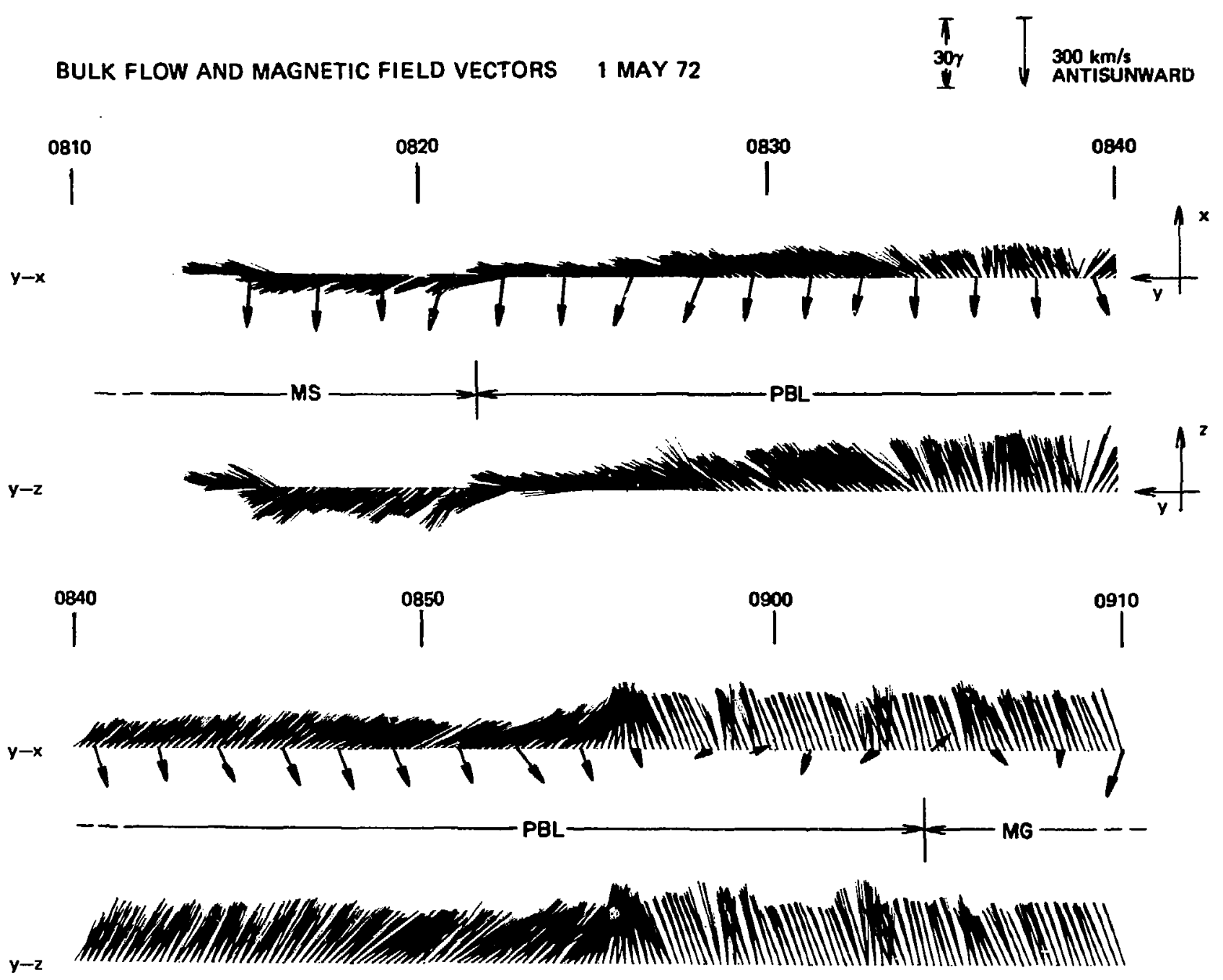


5 May 1972 IMP 6 Crossing

This low-latitude dawn-side crossing has an indistinct field change near 2344 UT. Cross-field flow components are substantial al though tailward field draping is not clear. In the inner part of the PBL, the density fluctuates near $5 \mathrm{~cm}^{-3}$ concurrent with enhanced variations in flow direction. 


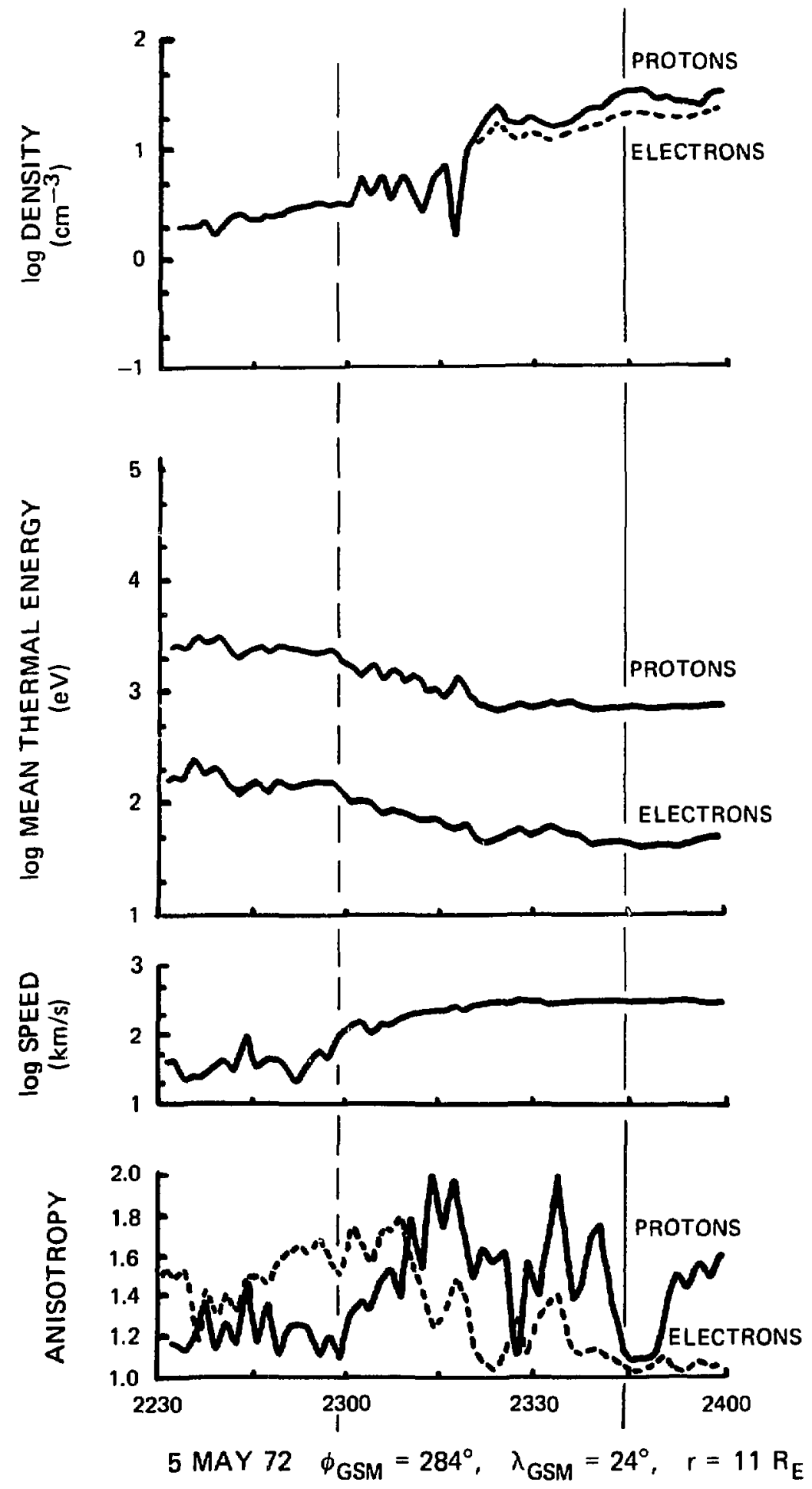



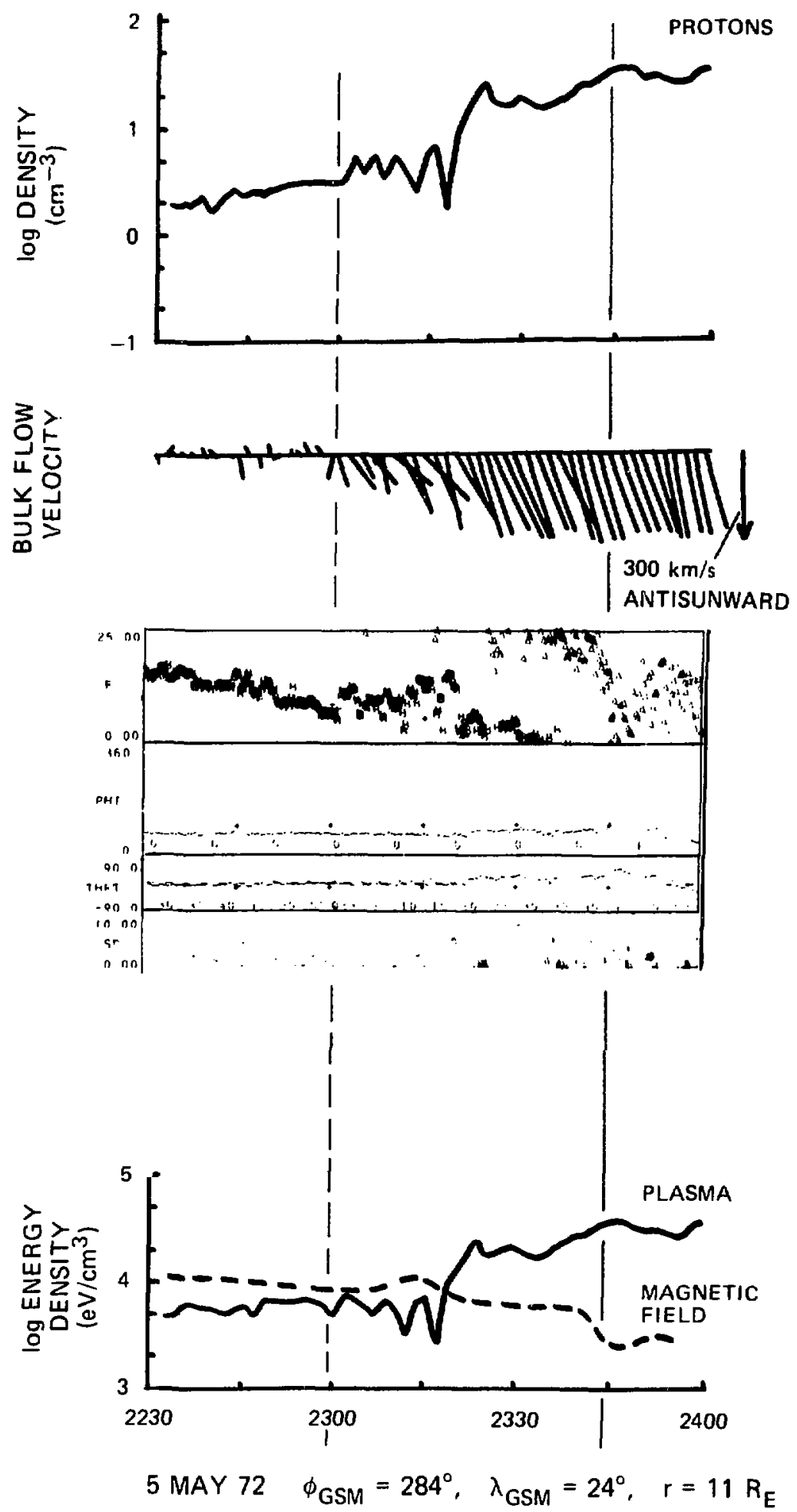

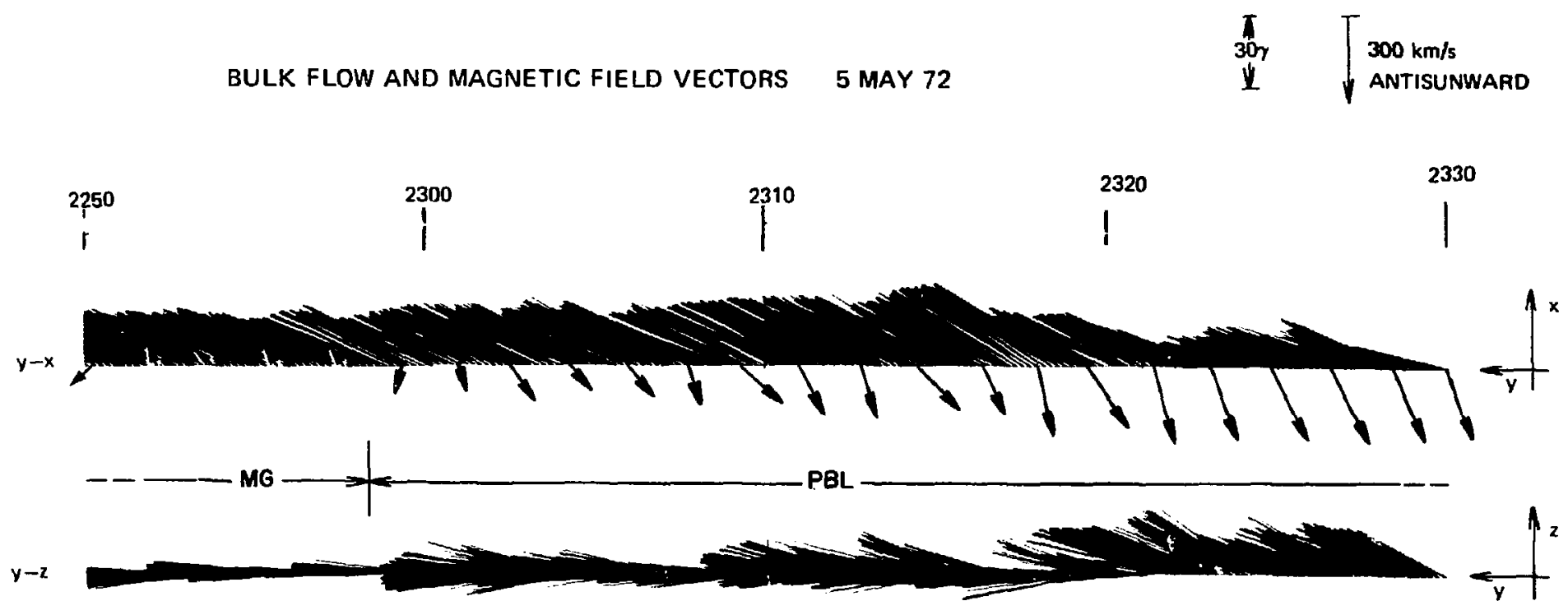
9 May 1972 IMP 6 Crossing

This low-latitude crossing is located on the dawn side of the outer cusp region. As with other crossings of the outer cusp region, the flow field is highly variable. Flow vectors in the PBL that have a sunward flow component are directed into the cusp. High ion anisotropies and density depletion are present in the nearby magnetosheath. Two isolated plasma regions with high density and field magnitude values are located in the nearby magnetosphere. their relationship to ths PBL is not clear because of the limitations of single satellite measurements. 


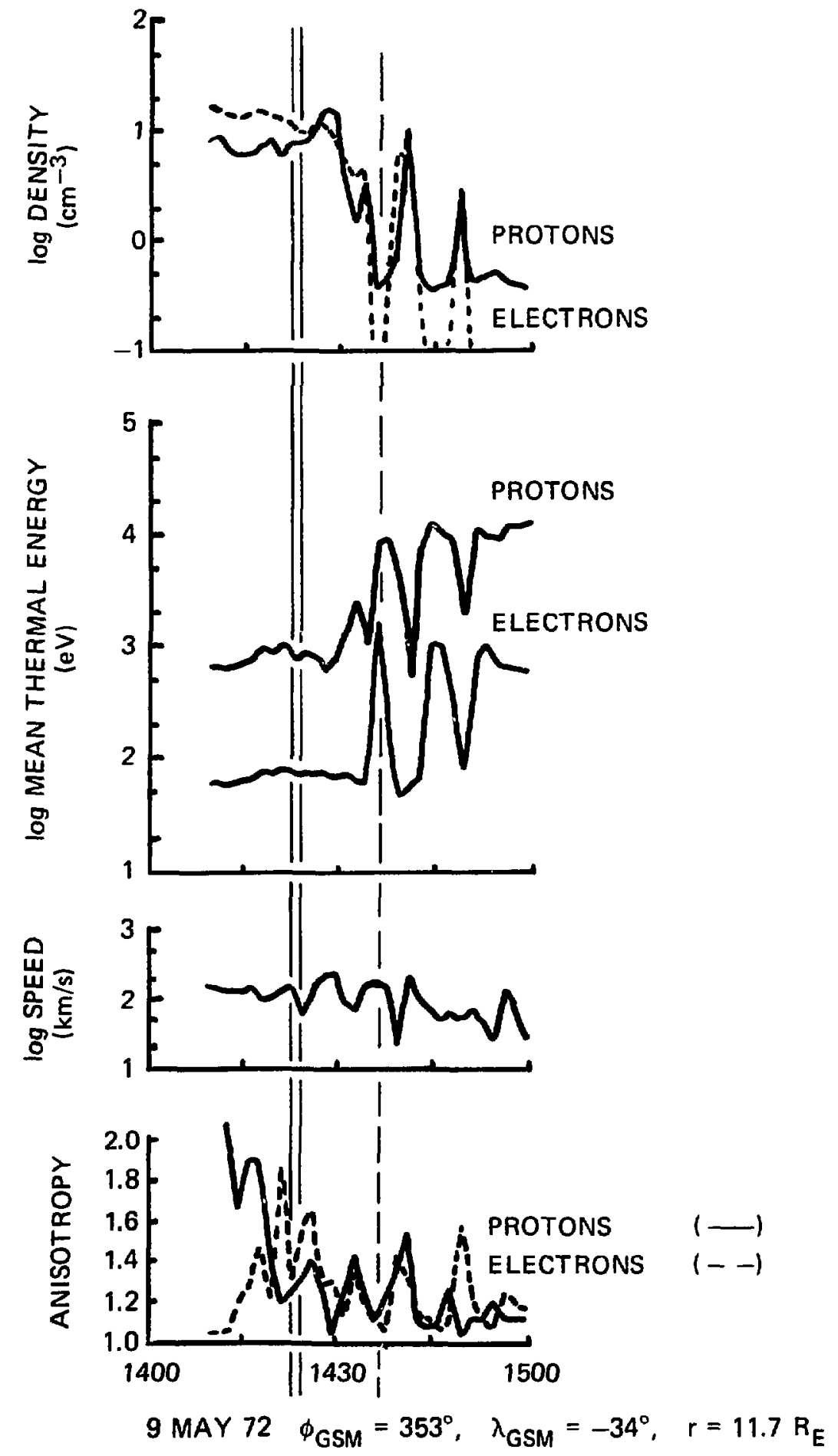




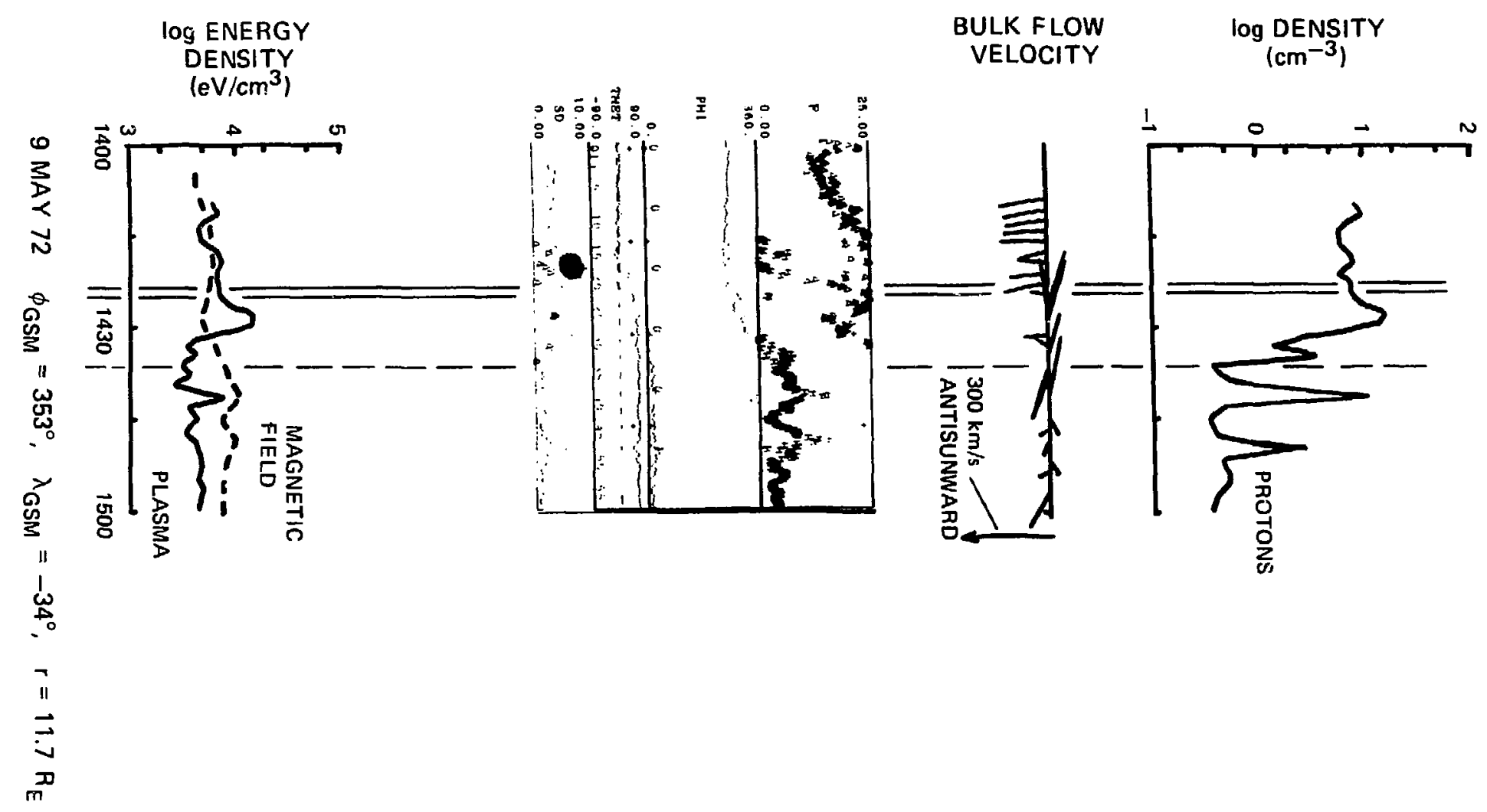




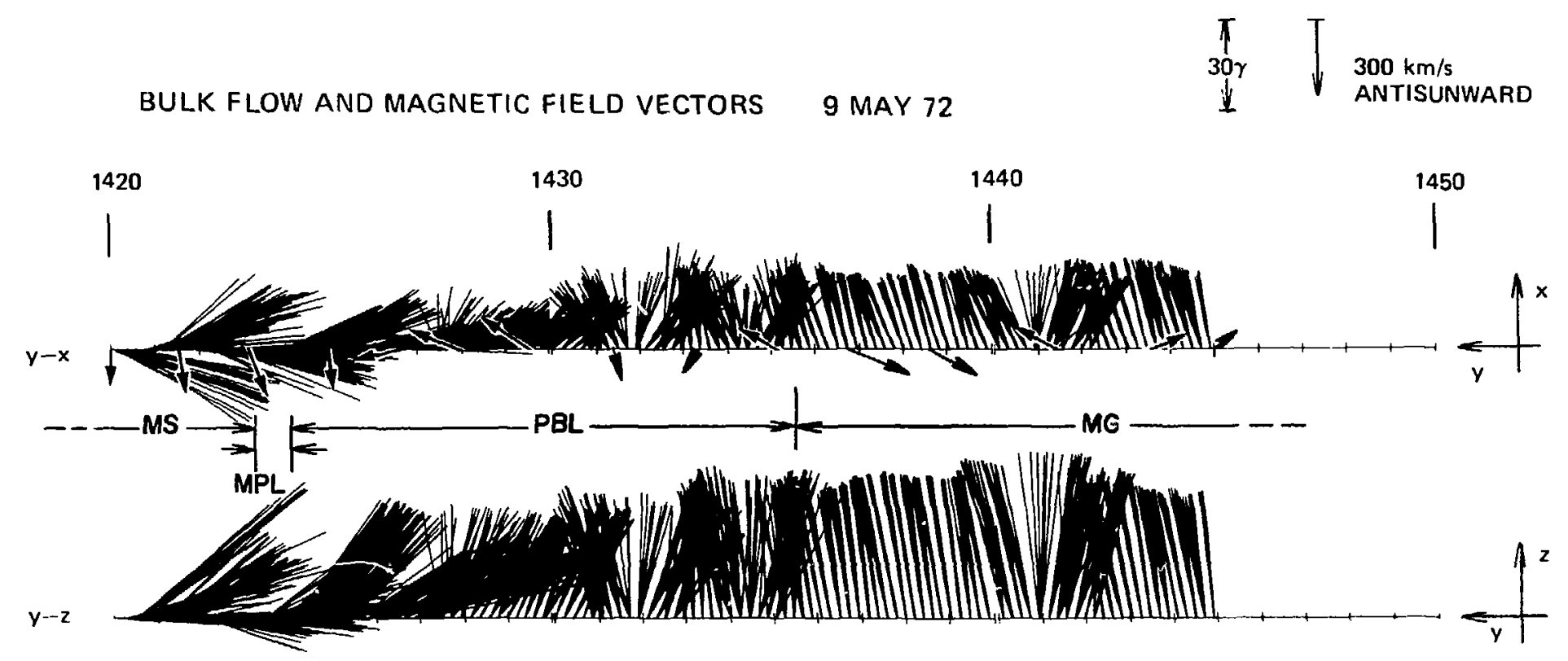


22 January 1973 IMP Crossing

This dusk-side high-latitude crossing does not show a single distinct MPL, al though the first possible position is marked on the figures. Significant cross-field plasma flow is present in the PBL with some noticeable taliward field draping. In comparison with crossings of the outer cusp region, the plasma flow field in this crossing is steadier and has a purely antisunward component. Field fluctuations are enhanced near the MPL similar to many other MPL-PBL crossings. 


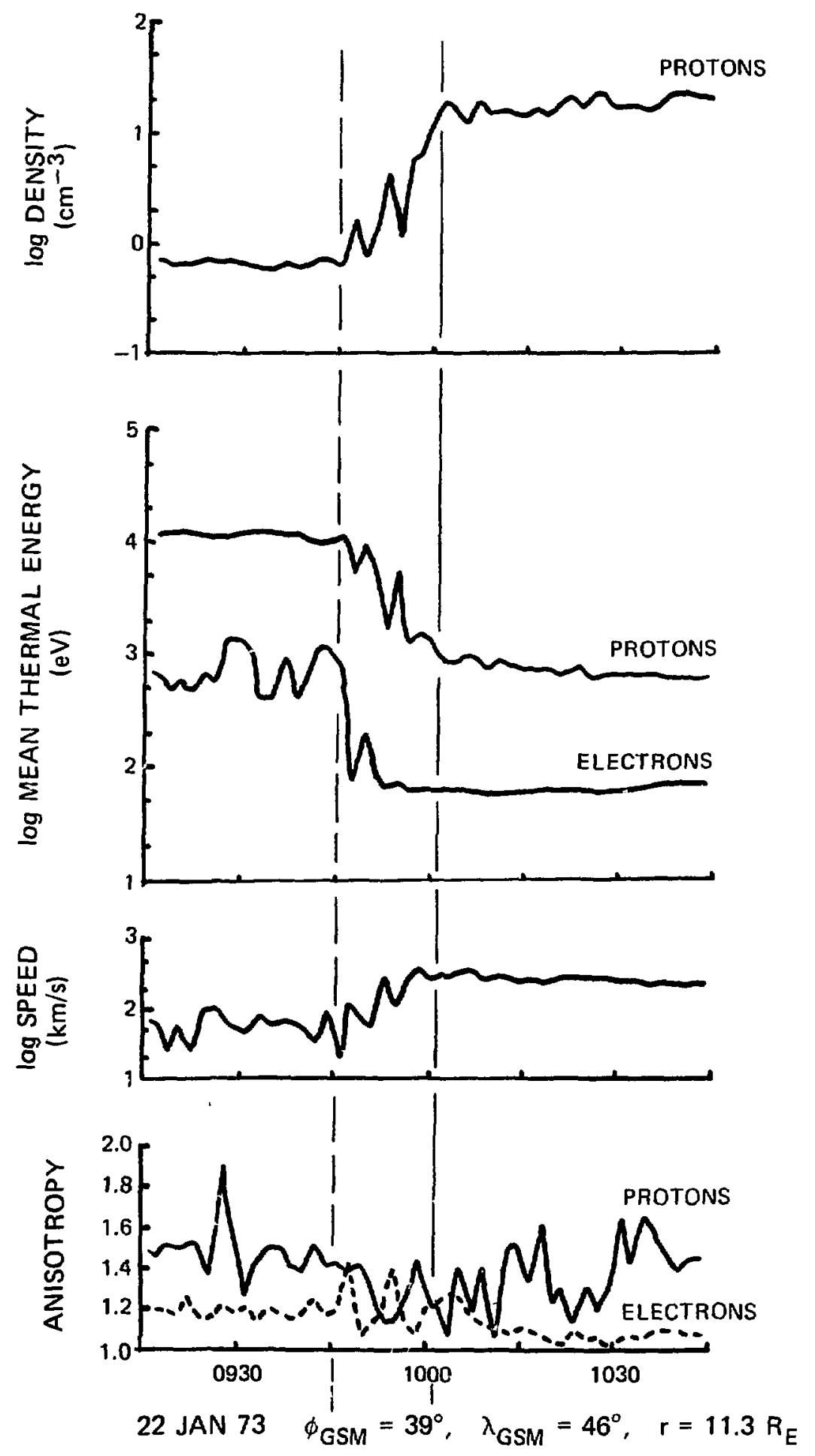




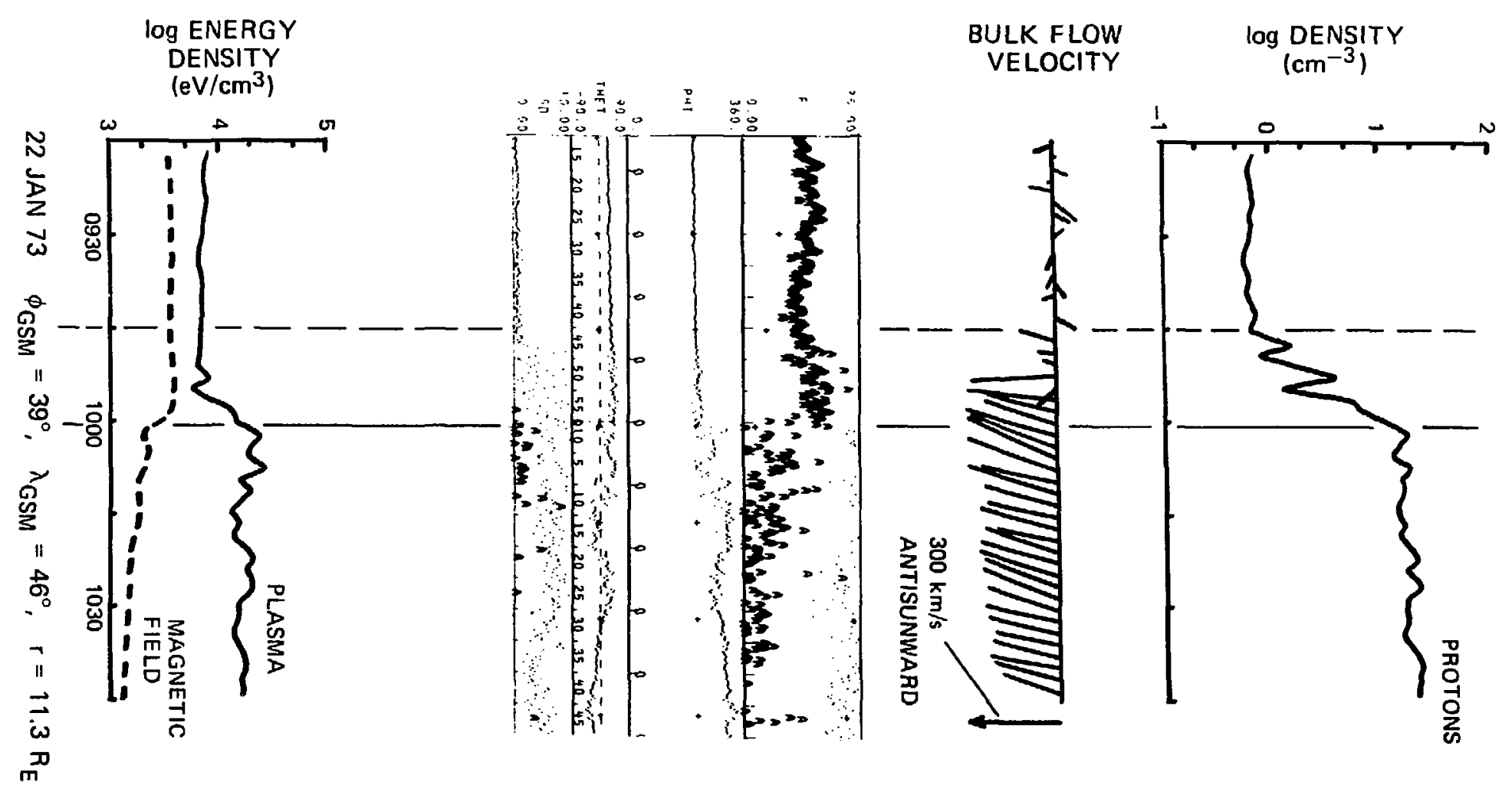

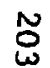



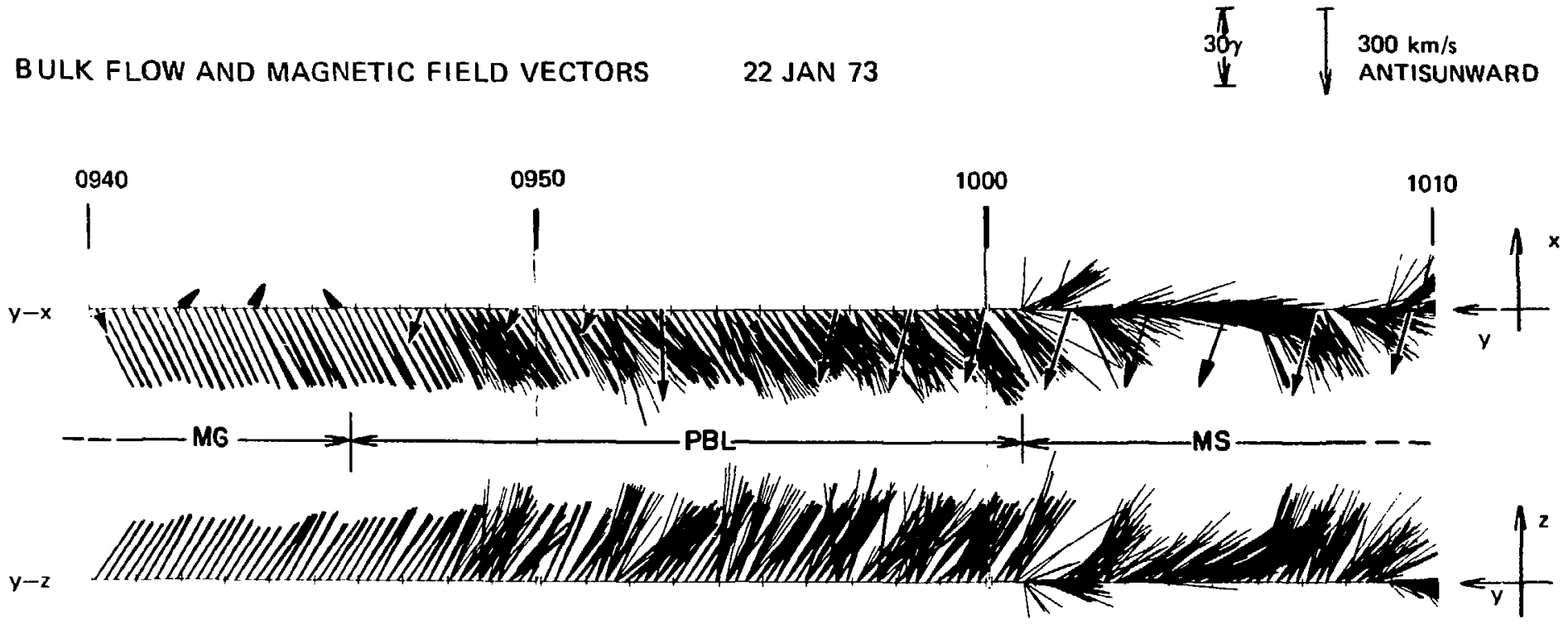


\section{February 1973 IMP 6 Crossing}

This long dusk-side crossing of the MPL and PBL shows a field discontinuity at $\backsim 1331$ UT which we tentatively identify as the MPL. The plasma density shows no distinctive change near the MPL and does not begin to drop toward the magnetospheric level until $\sim 10$ minutes later, when the other plasma parameters al so begin their primary changes. Plasma and field values do not mark a clear inner PBL location. This lack of a sharp inner boundary is relatively common in the IMP 6 data. An indistinct inner boundary is consistent with intermixing of magnetospheric and magnetosheath-like plasma.

Plots of the plasma bulk flow velocity and magnetic field show that a significant cross-field flow component of up to $\Omega 45^{\circ}$ is present in the PBL. The energetic electron (47- to $350-\mathrm{keV}$ ) intensity is similar in the PBL and the nearby magnetosphere and the pitch-angle distributions are also similar. In the dusk side outer magnetosphere the energetic electron pitch angle distributions are not expected to retain a pancake-shaped distribution due to the intersection of drift paths with the magnetopause (West, 1973). The marked similarity of magnetospheric and PBL pitch angle distributions suggest that the PBL is on closed field lines. In contrast, the nearby magnetosheath shows an antisunward streaming of the energetic electrons. 

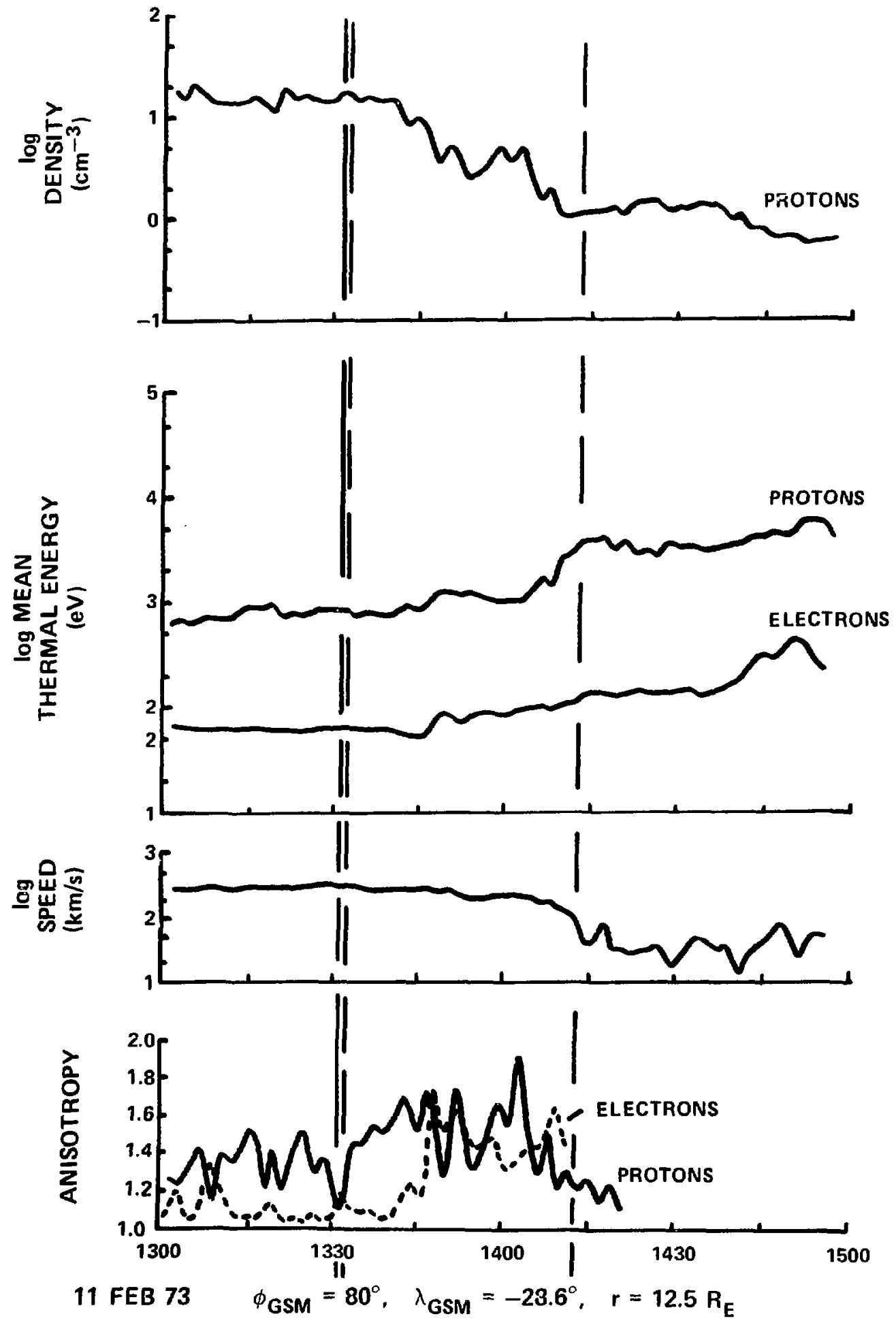


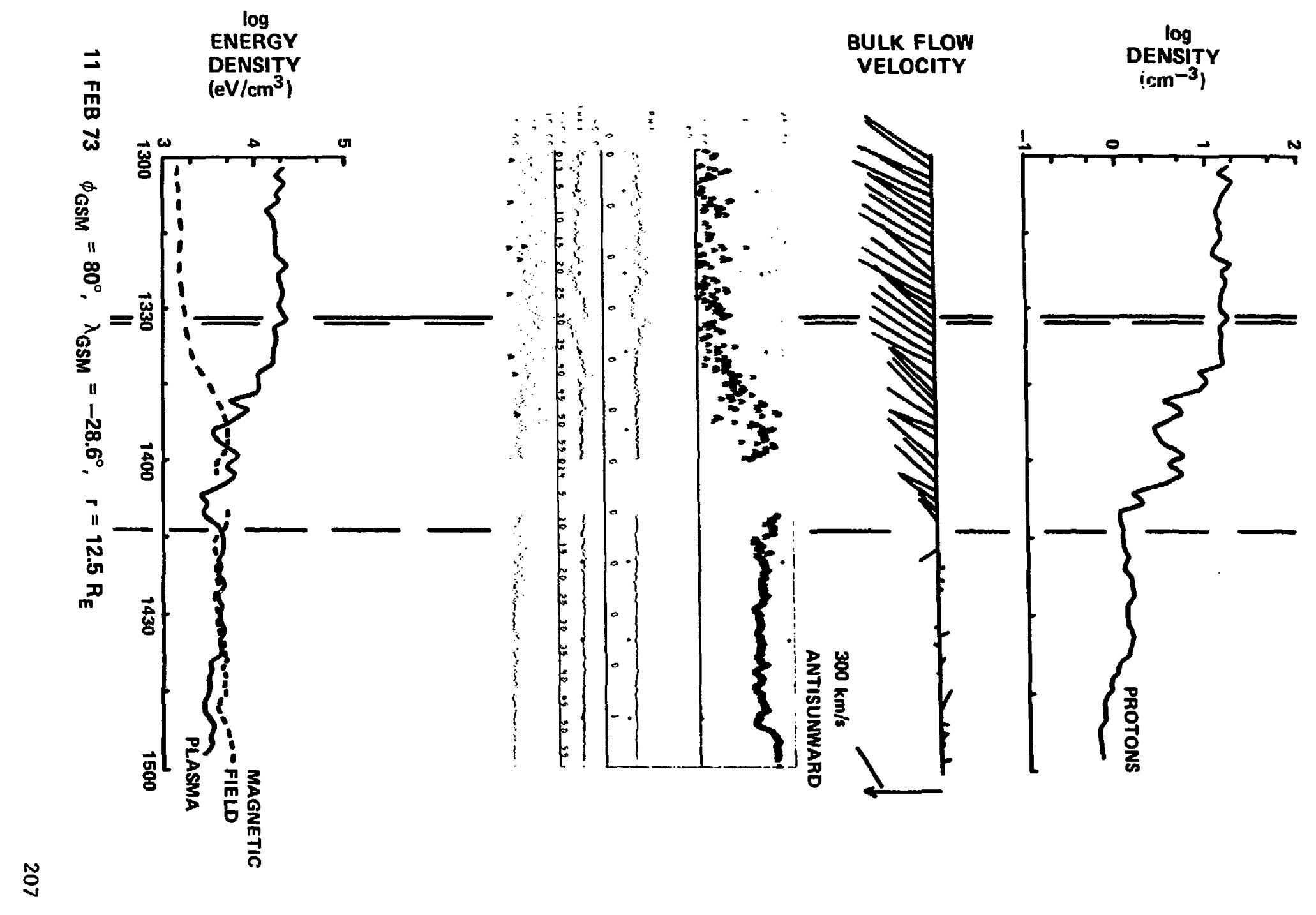


BULK FLOW AND MAGNETIC FIELD VECTORS 11 FEB $73 \quad \begin{array}{cc}\pi & 30 \gamma \\ 1 & 300 \mathrm{~km} / \mathrm{s}\end{array}$
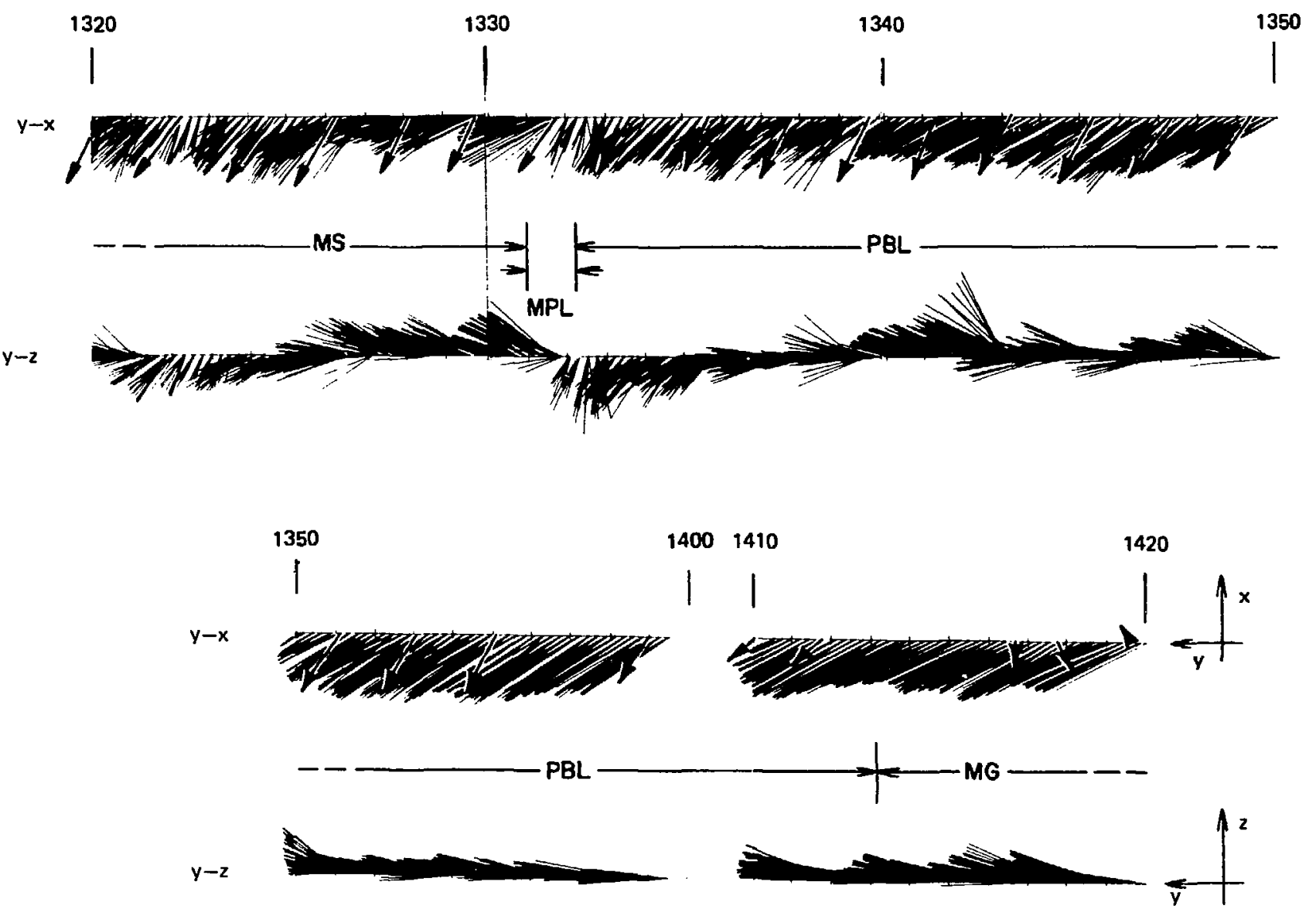
ENERGETIC ELECTRON (47- to $350-\mathrm{keV}$ )

PITCH-ANGLE DISTRJBUTIONS, 11 FEBRUARY 1973

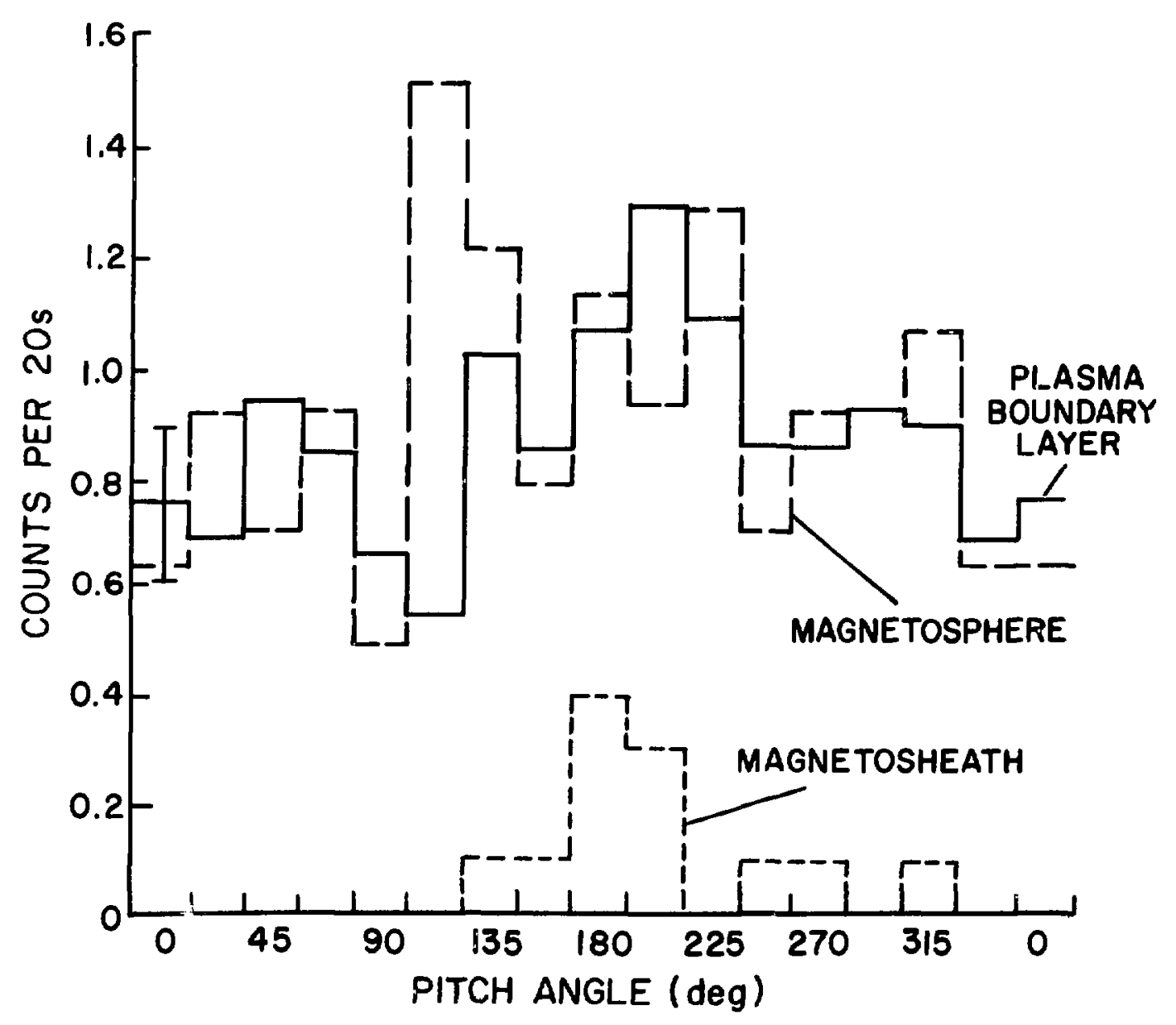


19 February 1973 iMP ó crossing

This low-latitude dusk-sije crossing shows signifiant cross-field bulk plasma flow in the PBL. A high-density plasma region near 2215 UT Includes one flow vector with a sunward somponent. The relationship of this region to the PBL cannot be evaluated adequately based on these single satellite measurements. A tailward streaning distribution of energetic electron is observed in the nearby magnetosheatn. Pitch-angle distributions for the $\mathrm{PBC}$ and nearby magnetosphere are both essentially isotropic as expected at low latitudes near the dusk-side magnetospheris boundary.

ENERGETIC. ELECTRON (47- to $350-\mathrm{keV}$ )

PITCH-ANCLE DISTRIBUTIONS, 19 FEBRUARY 1973

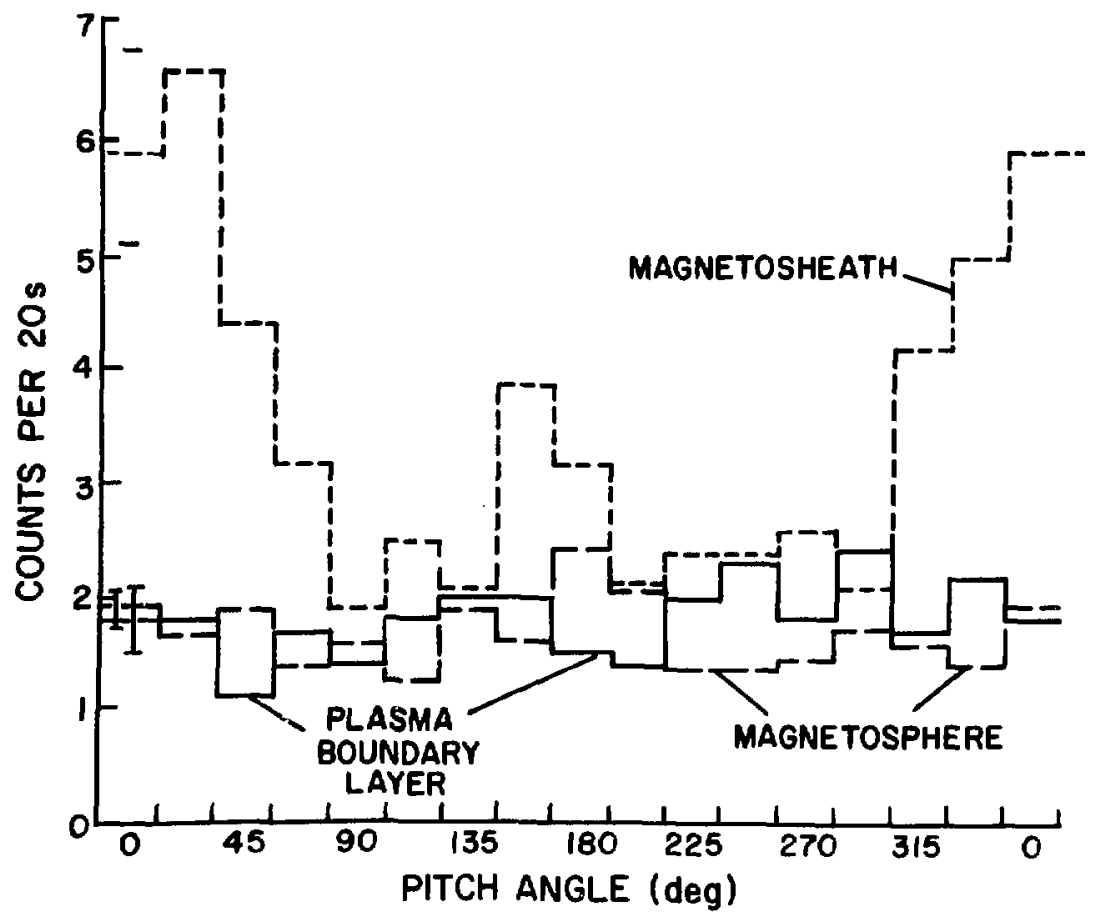




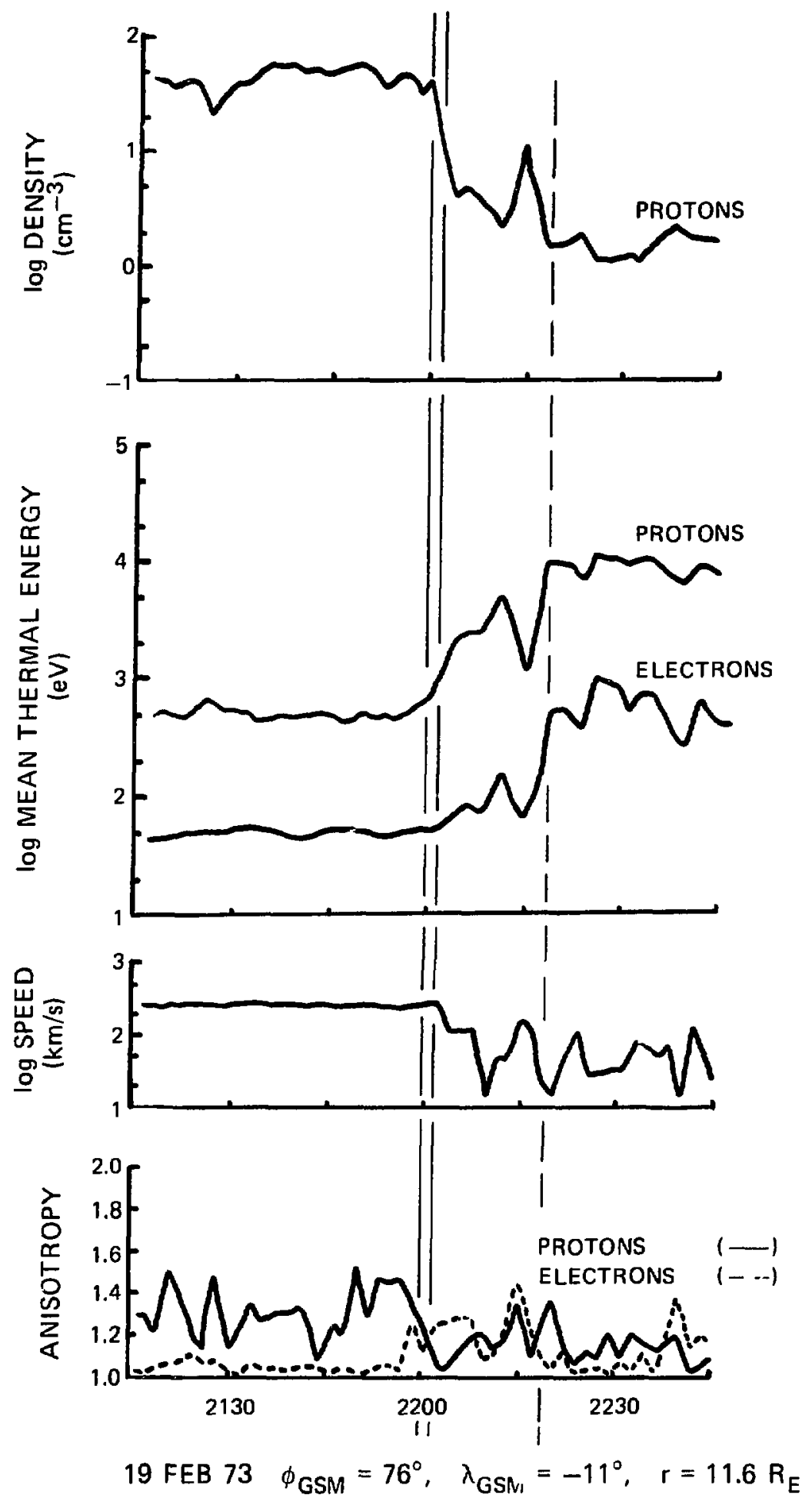




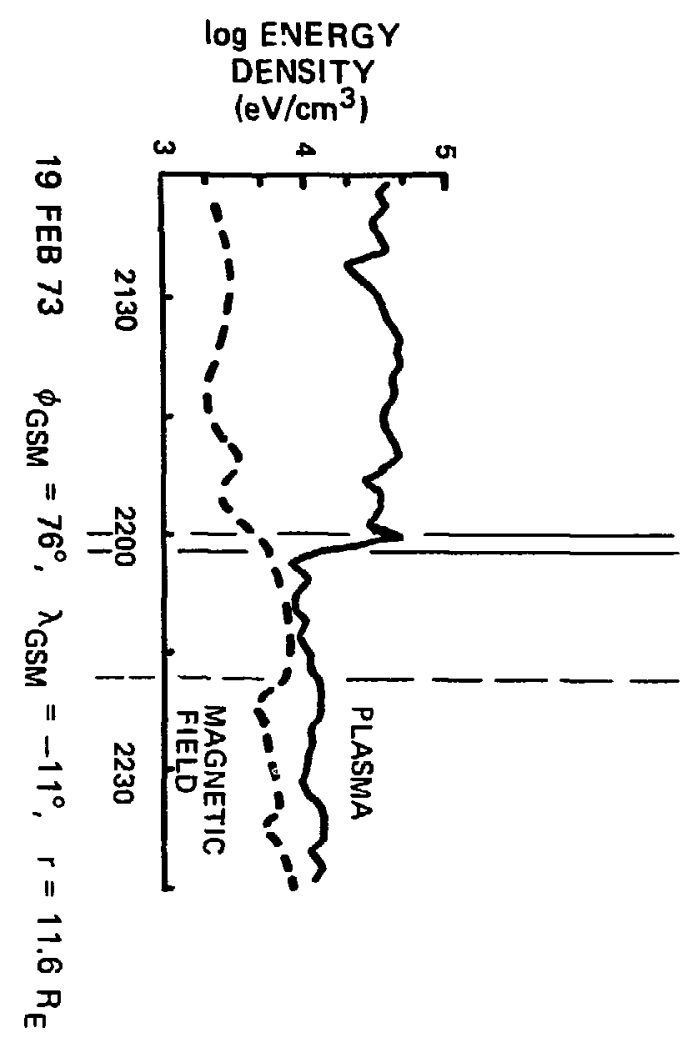

BULK FLOW
VELOCITY

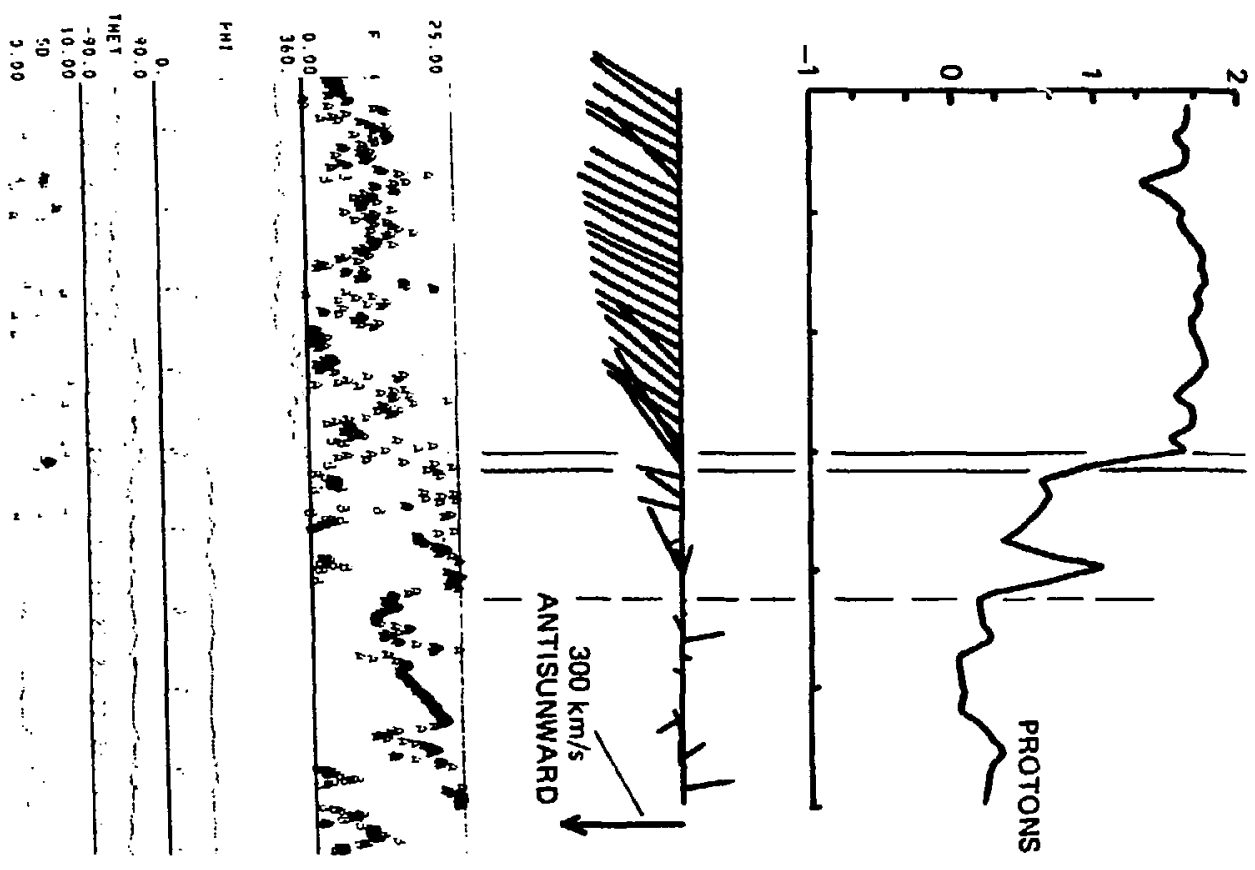


BULK FLOW AND MAGNETIC FIELD VECTORS 19 FEB 73

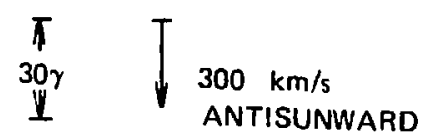

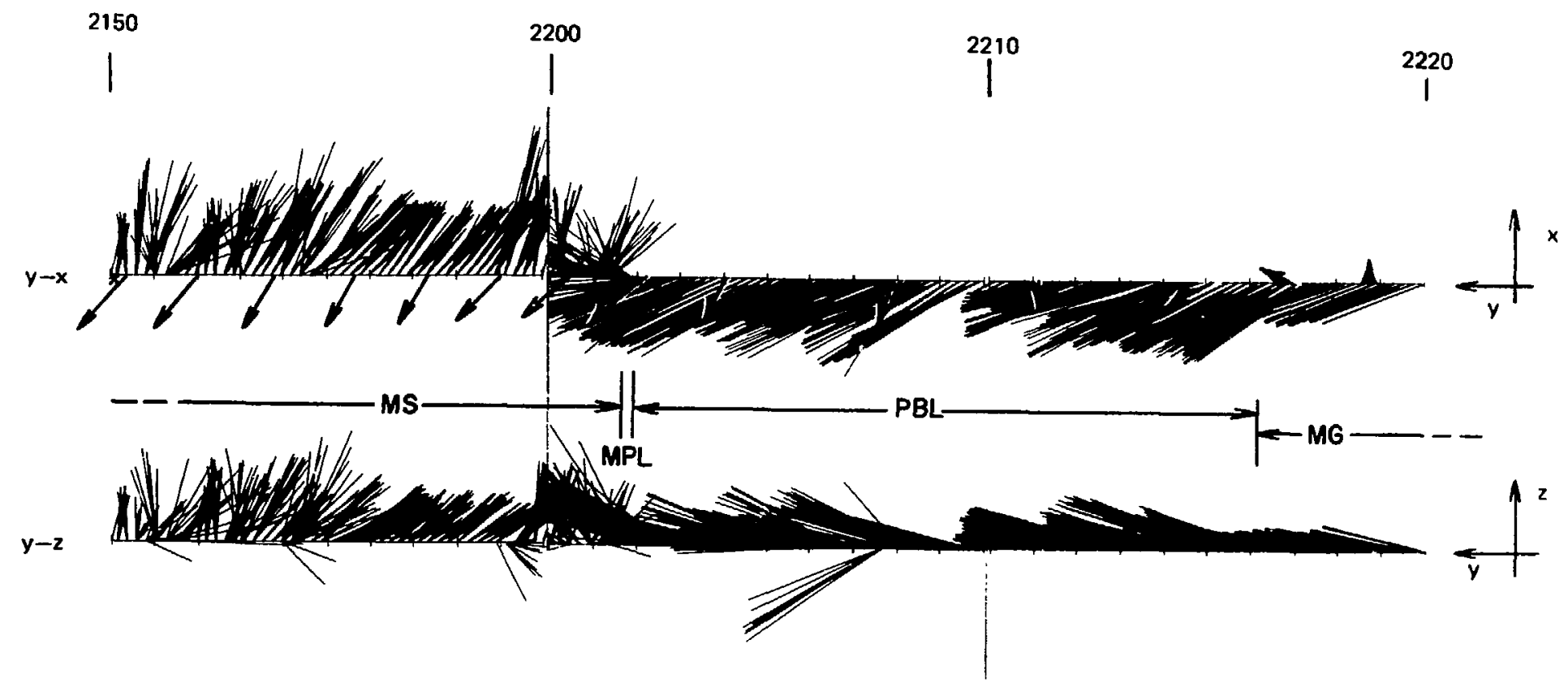


8 March 1973 IMP 6 Crossing

This low-latitude dusk-side crossing has variable bulk plasma flow with significant cross-field components at times. The region of reduced density near 1245 UT may be a reentry of the PBL. Near the MPL, the shift in flow direction is larger than usual. Except for the flow shift, the MPL is only marked by a change in direction of the magnetic field. 


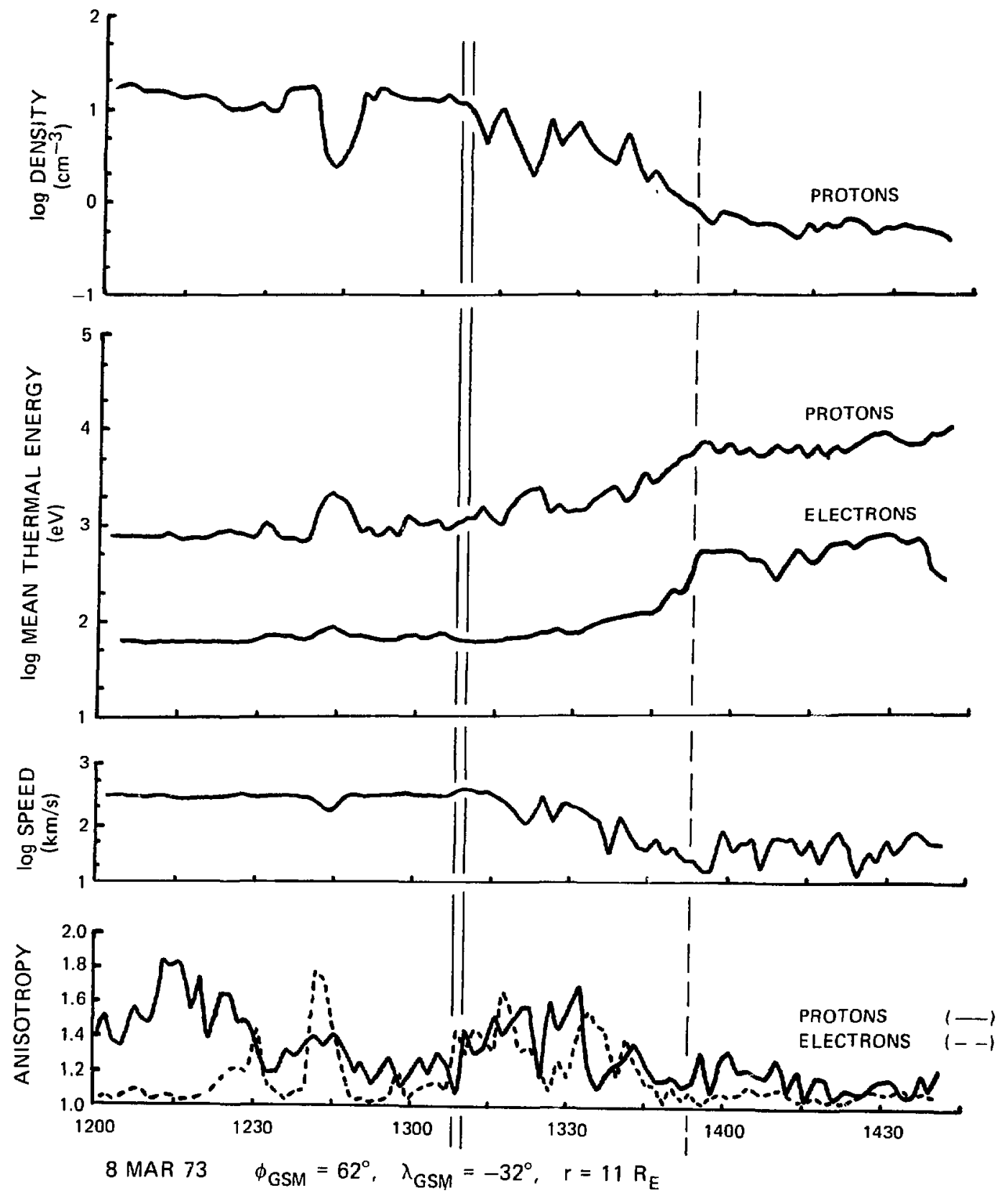



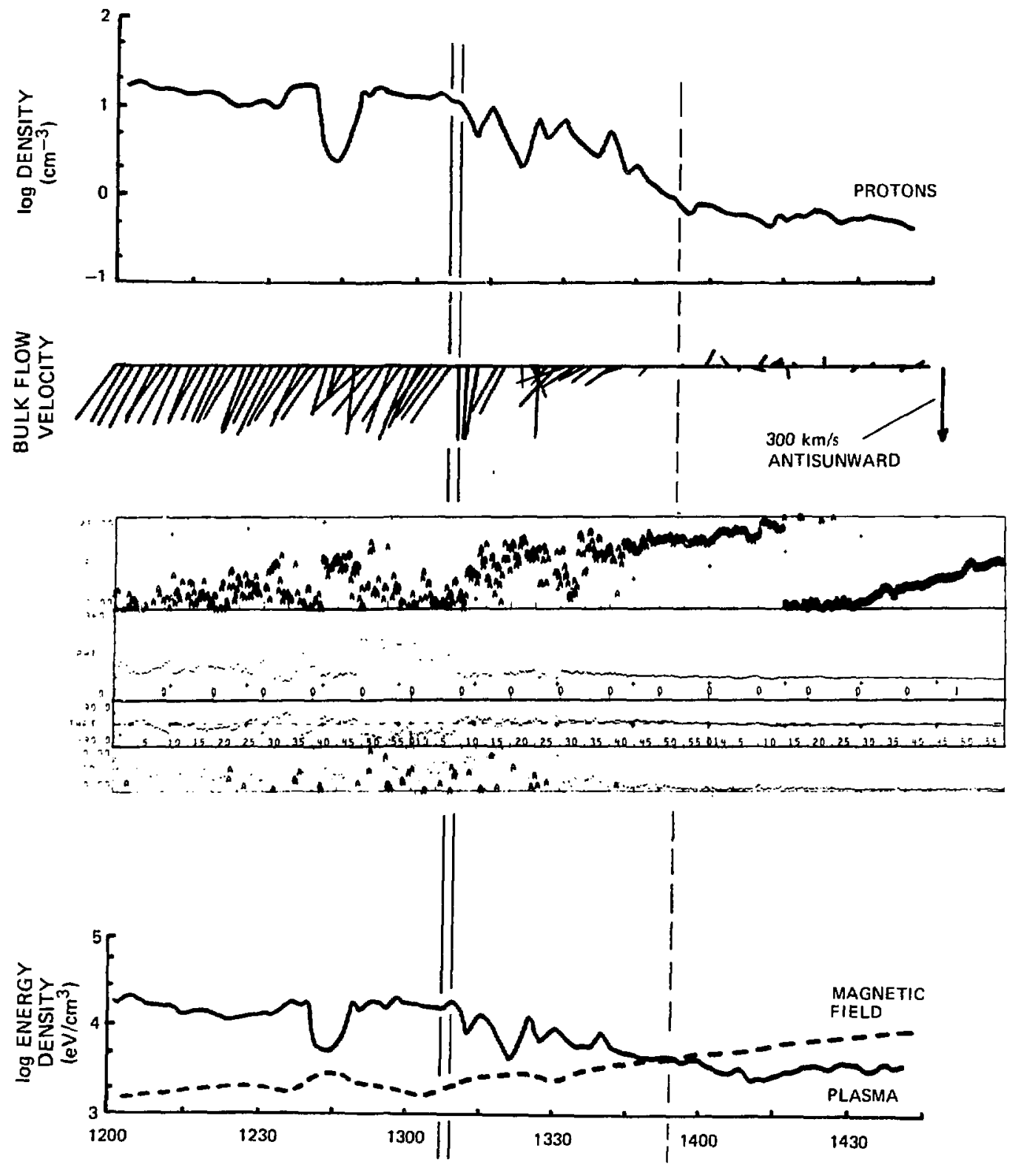

8 MAR $73 \phi_{\mathrm{GSM}}=62^{\circ}, \quad \lambda_{\mathrm{GSM}}=-32^{\circ}, \quad r=11 \mathrm{R}_{\mathrm{E}}$ 

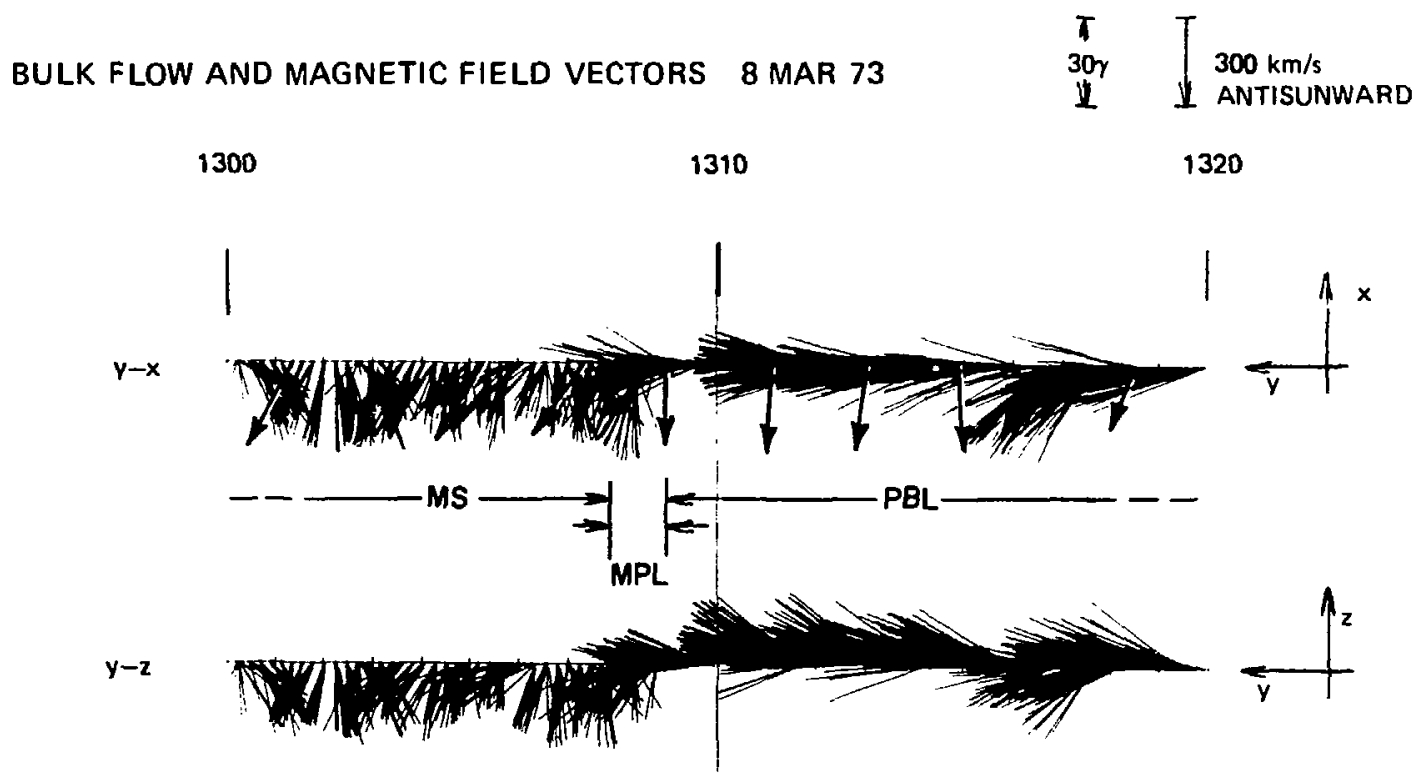

1320

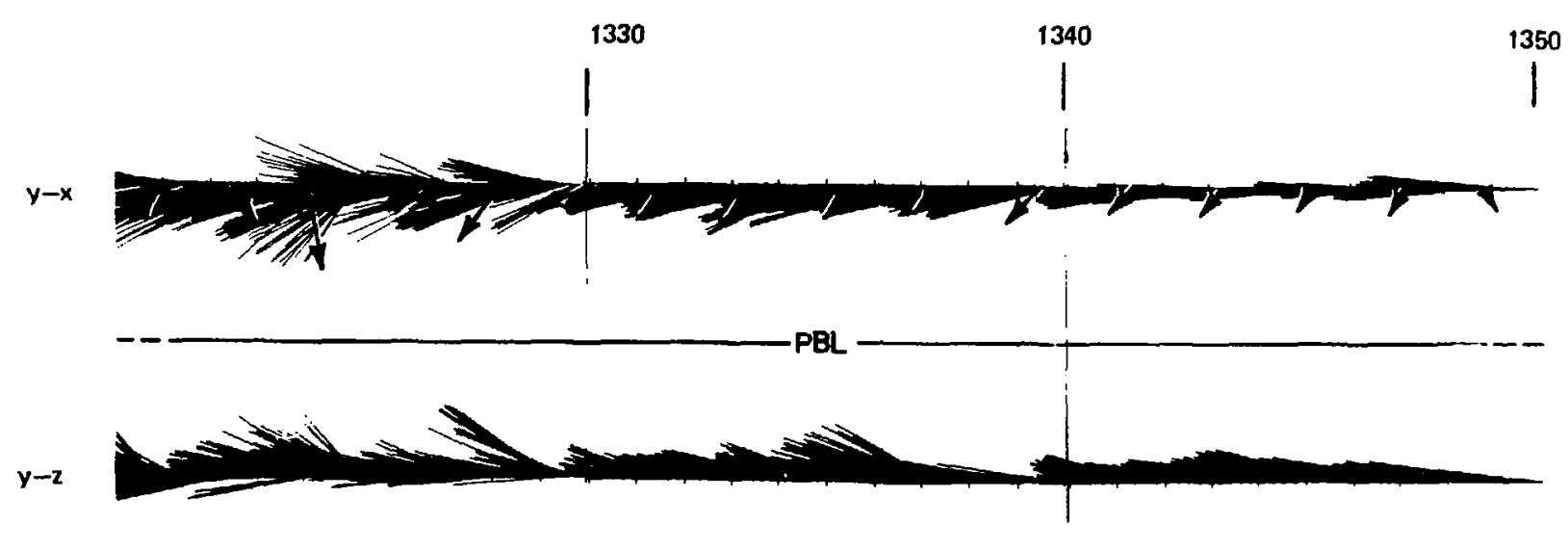


25 March 1973 IMP 6 Crossing

This low-latitude dusk-side crossing has a very unusual sunward component flow vector located near the MPL. Another crossing of the MPL occurs before $0400 \mathrm{UT}$. The PBI flow field is variable but not unusually so for this location. A detached plasma region convected sunward from the magnetotall may account for this sunward flow component (c.f. the 19 Feb 1973 crossing). The bulk plasna flow field is generally fieldal Igned in the PBL, at least more so than is often observed. 


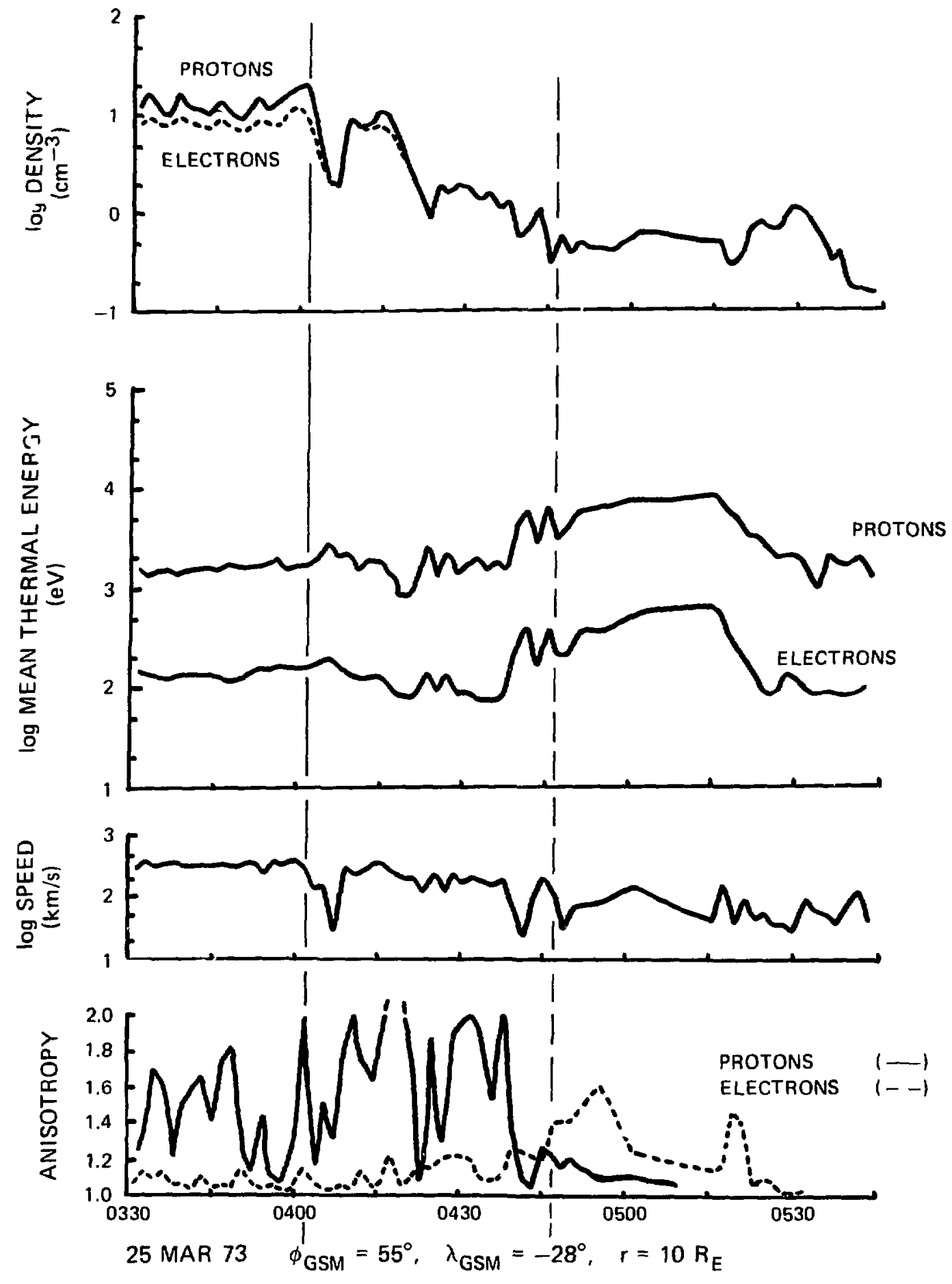




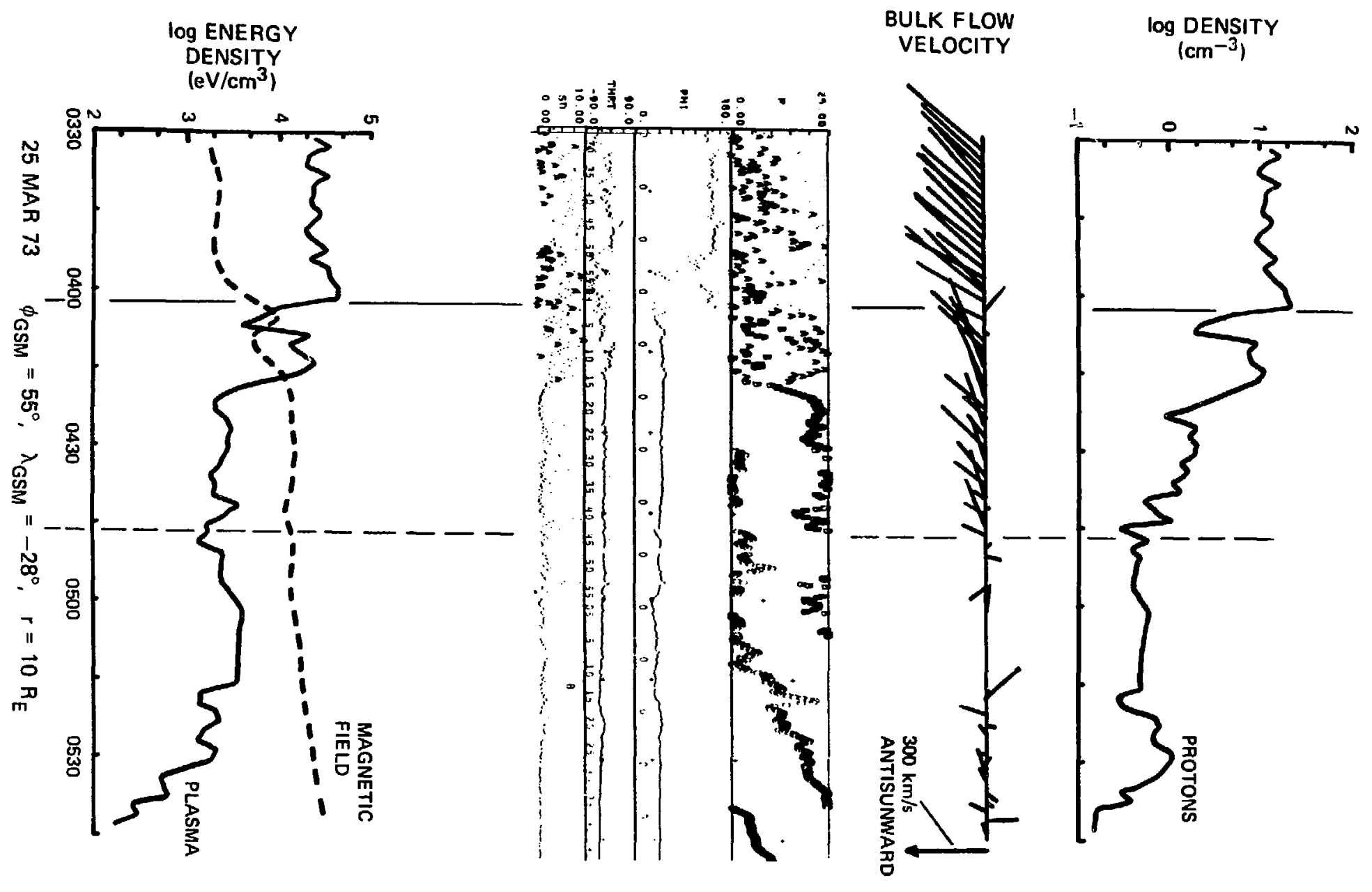



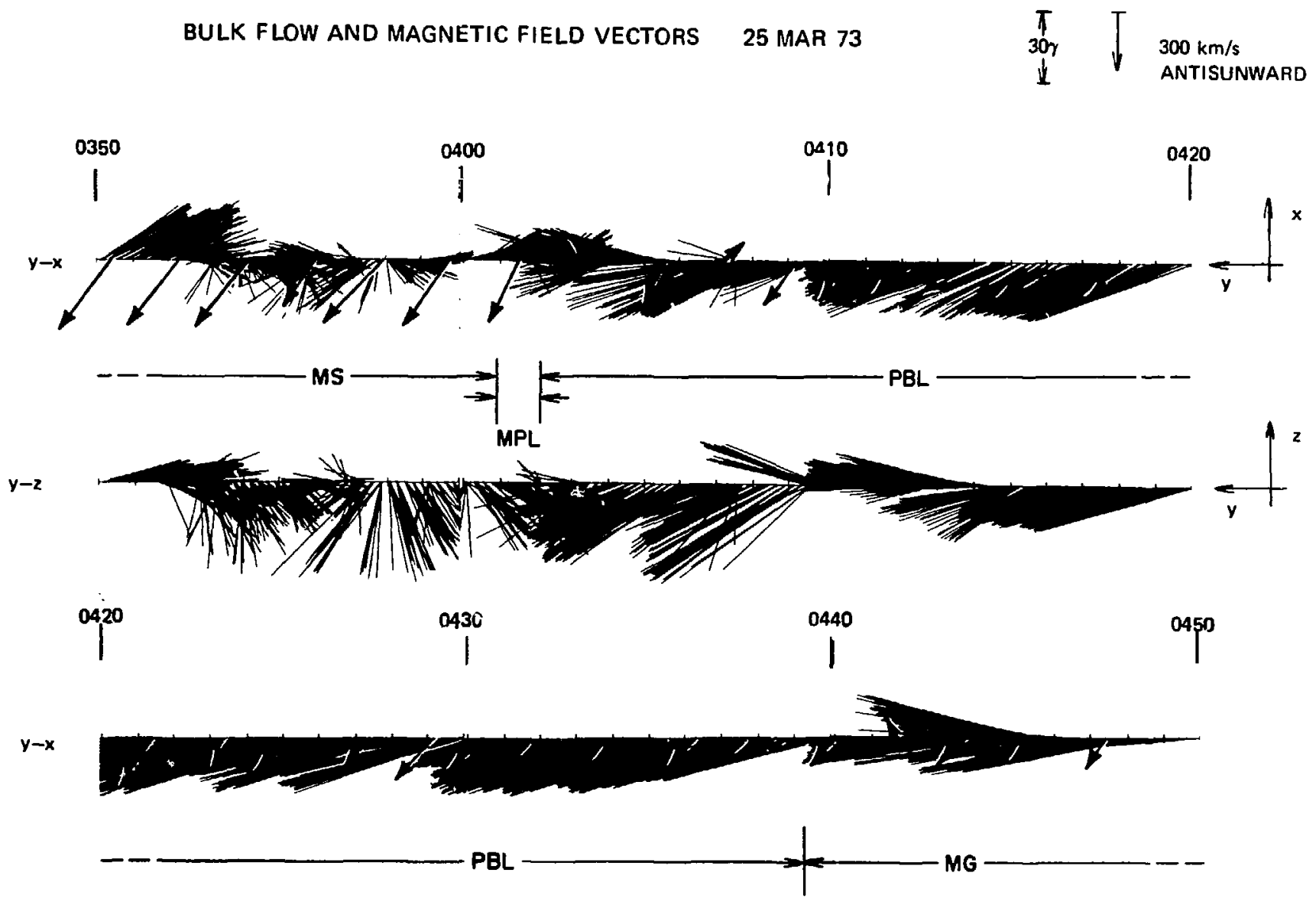

$y-2$

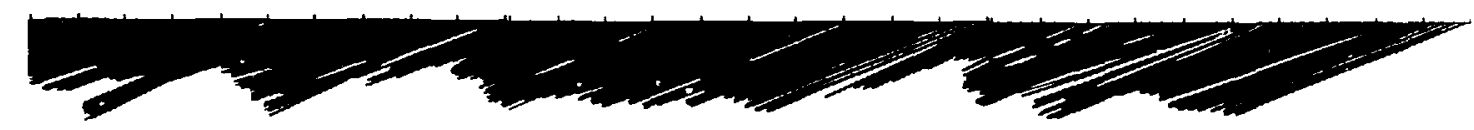


11 April 1973 IMP 6 Crossing

This low-latitude dawn-side crossing has a very sharp MPL with significant cross-field flow components in the PBL. Energetic electron pitch-angle distributions in the PBL and nearby magnetosphere are pancake-shaped al though the nearby magnetosheath distribution is nearly isotropic. A notable sunward flow somponent appears in the nearby magnetosphere. The density decrease within the PBL is unusually high and measured flow vectors near this point are affected by this rapid transition.

ENERGETIC ELECTRON (47- to 350-keV)

PITCH-ANGLE DISTRIBUTIONS, 11 APRIL 1973

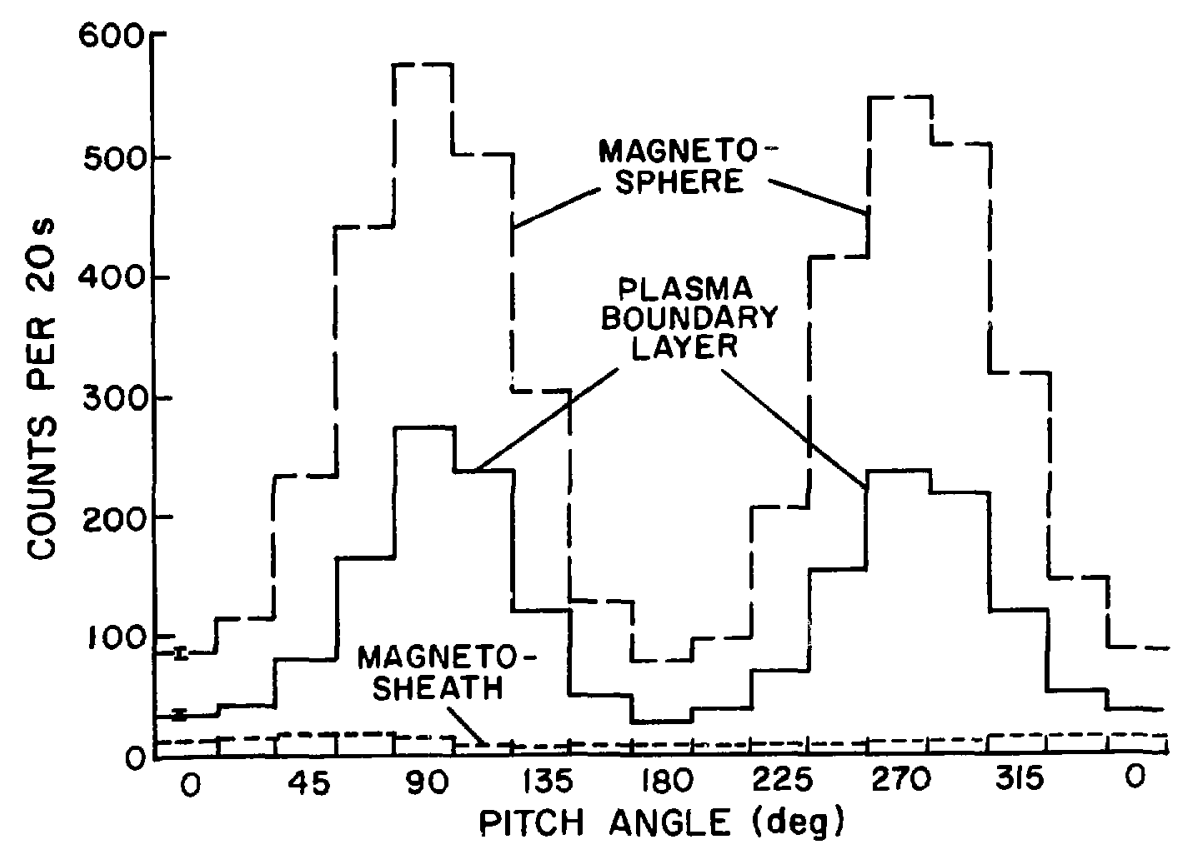




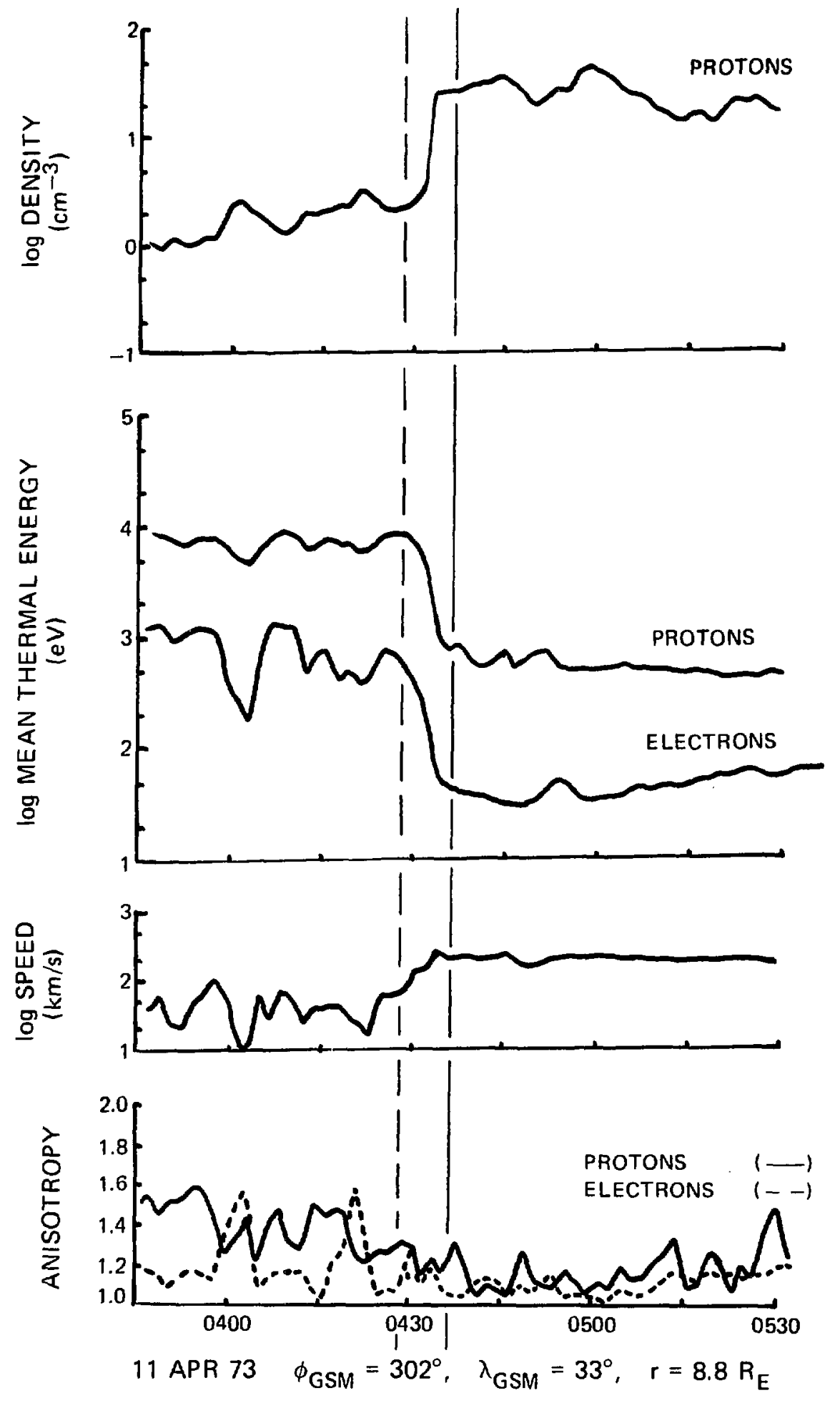




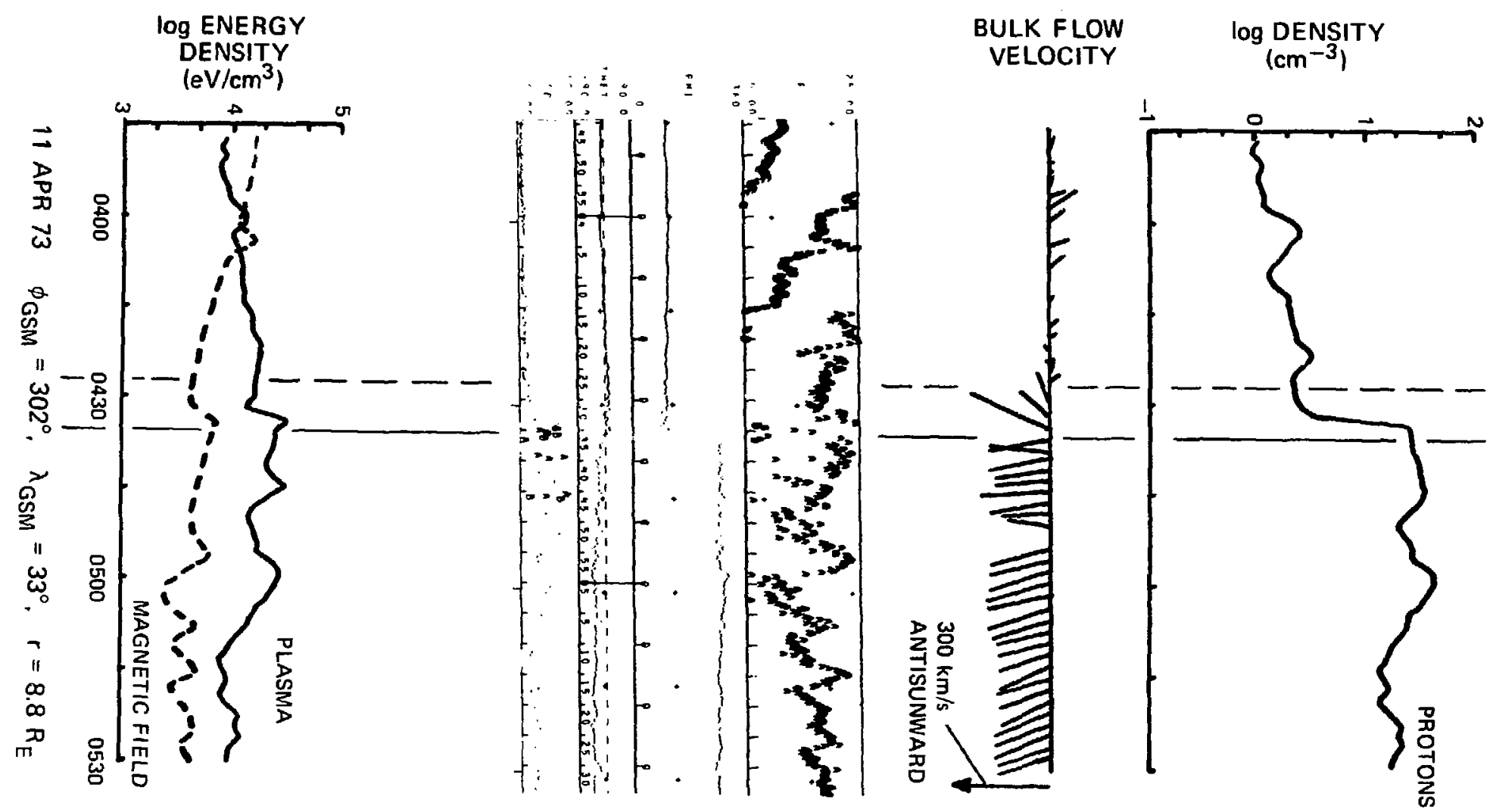



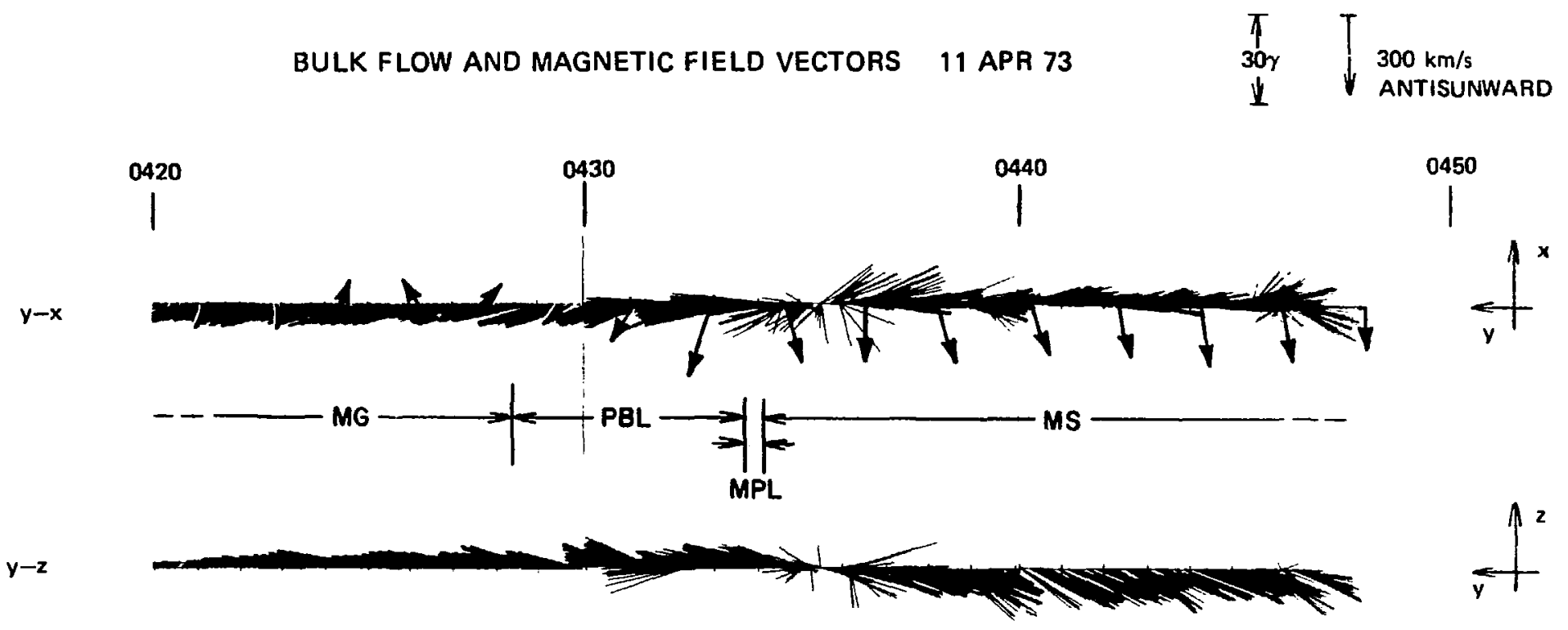
14 April 1973 IMP 6 Crossing

This low-latitude dusk-side crossing does not have any clear MPL location. Substantial cross-field flow components are present in the PBL. Plasma density and magnetic field magnitude show significant local enhancements within the PBL. 


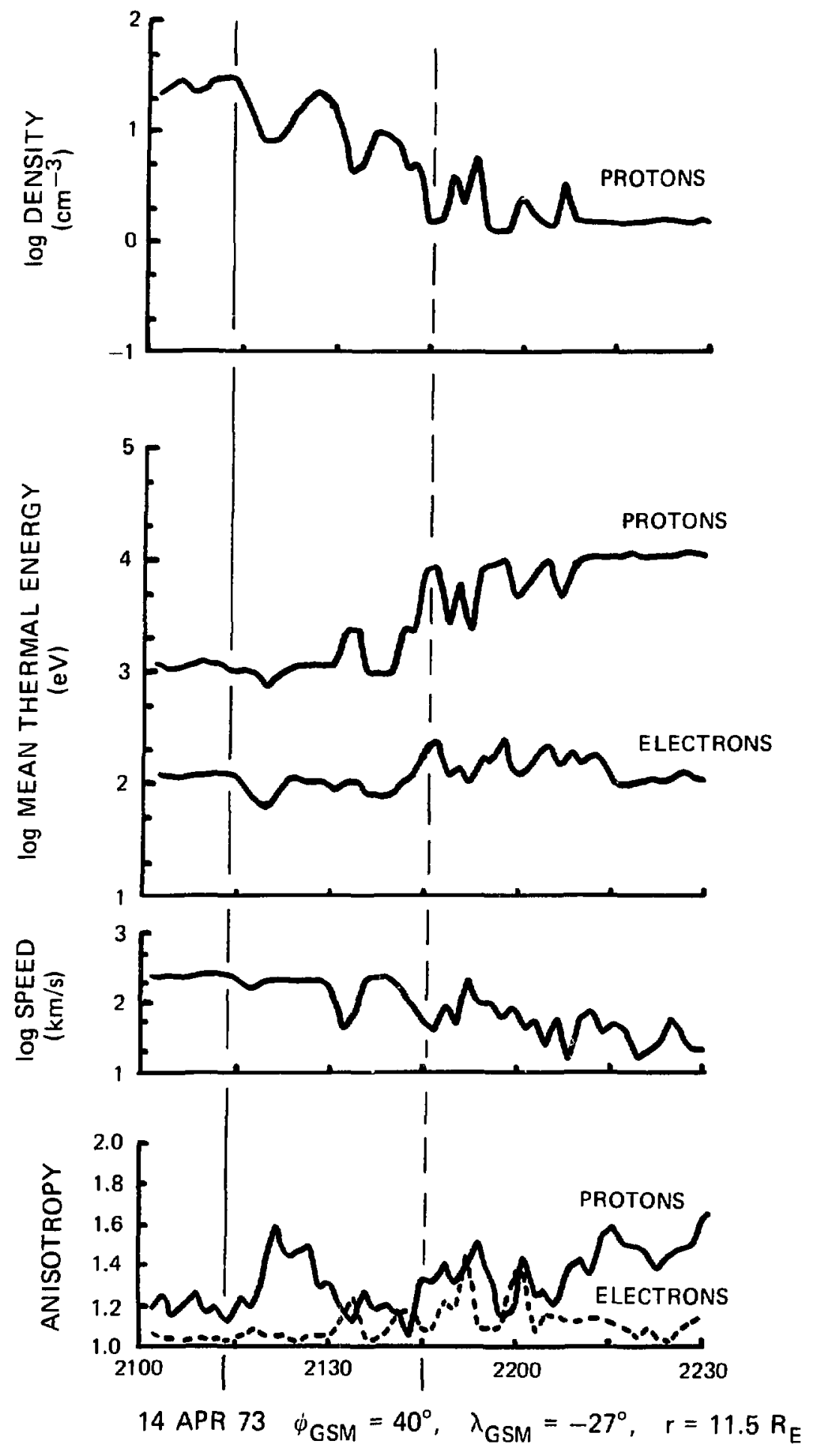




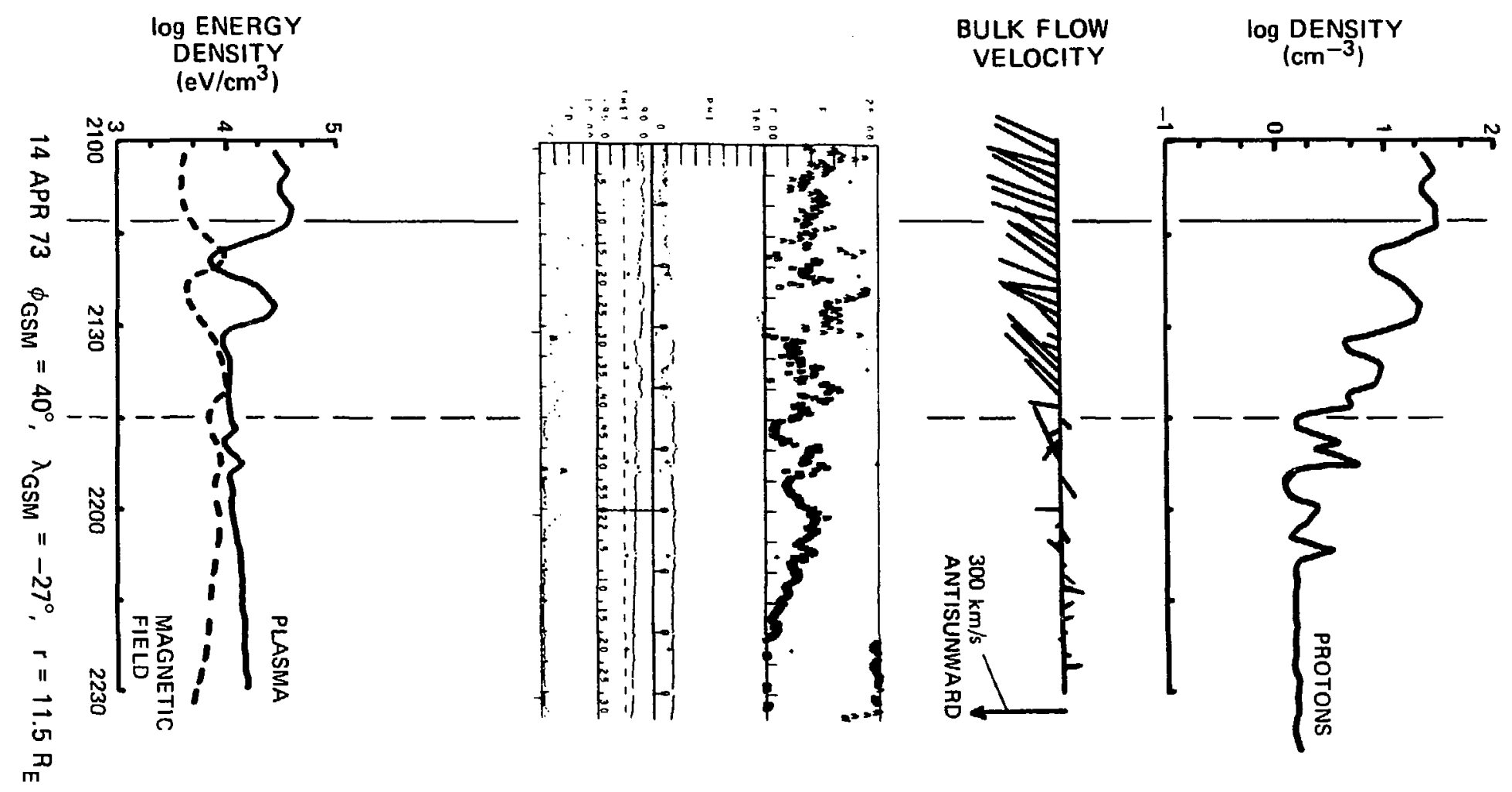




$$
\text { BULK FLOW AND MAGNETIC FIELD VECTORS } 14 \text { APR } 73 \quad \begin{gathered}
\pi \\
30 \gamma \\
\Downarrow
\end{gathered} \quad\left[\begin{array}{l}
300 \mathrm{~km} / \mathrm{s} \\
\text { ANTISUNWARD }
\end{array}\right.
$$

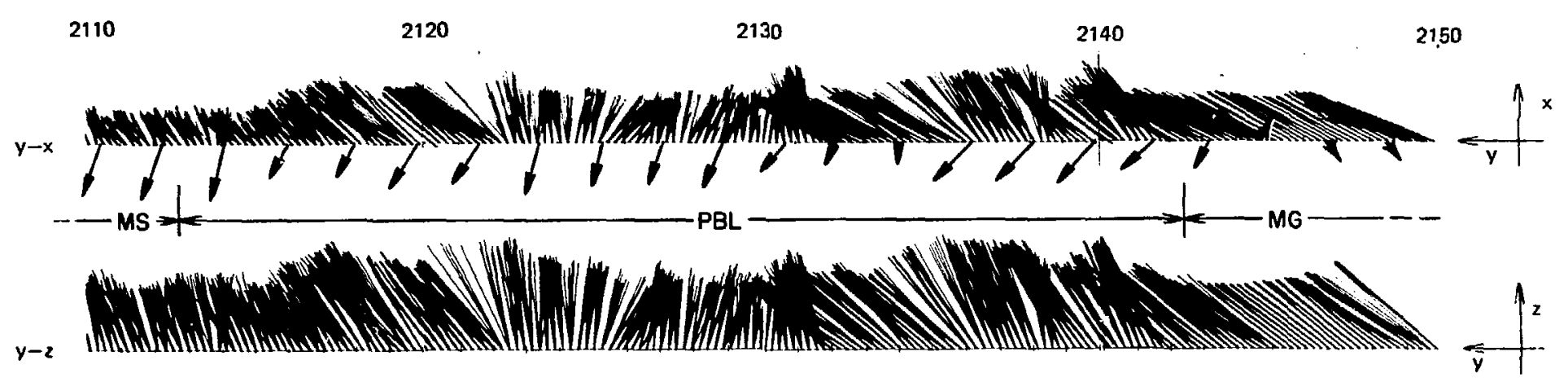


16 December 1973 IMP 6 Crossing

This dusk-side crossing is located near the equator at the dawn-dusk meridian. The bulk plasma flow is generally field-aligned in the PBL. A high-density isolated plasma region located at 0934 UT may represent only a reentry of the PBL al though a comparison of mean thermal energy and velocity values suggests that it may be a distinct plasma region. Most other plasma and field signatures for this crossing are very clear. 


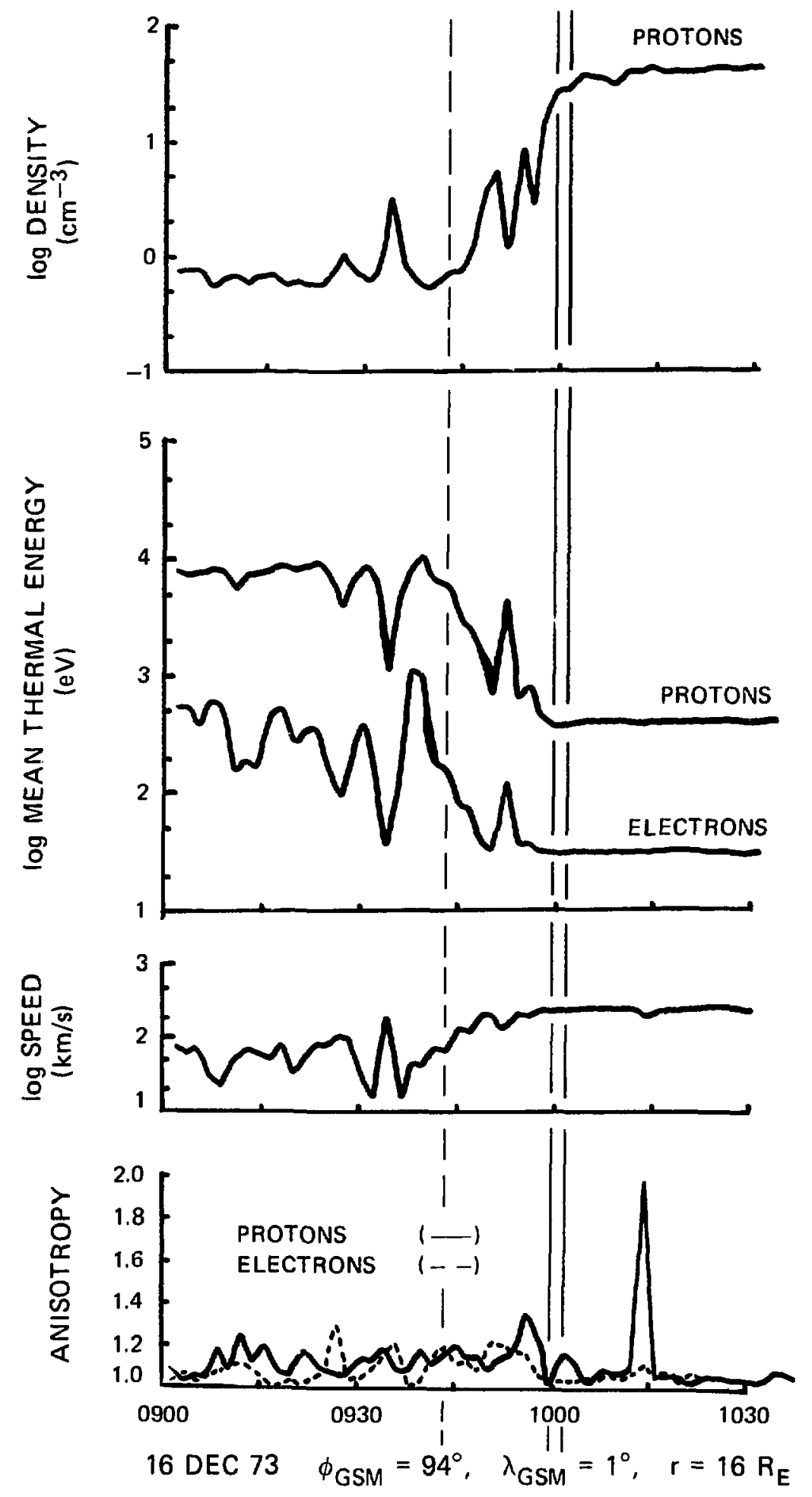




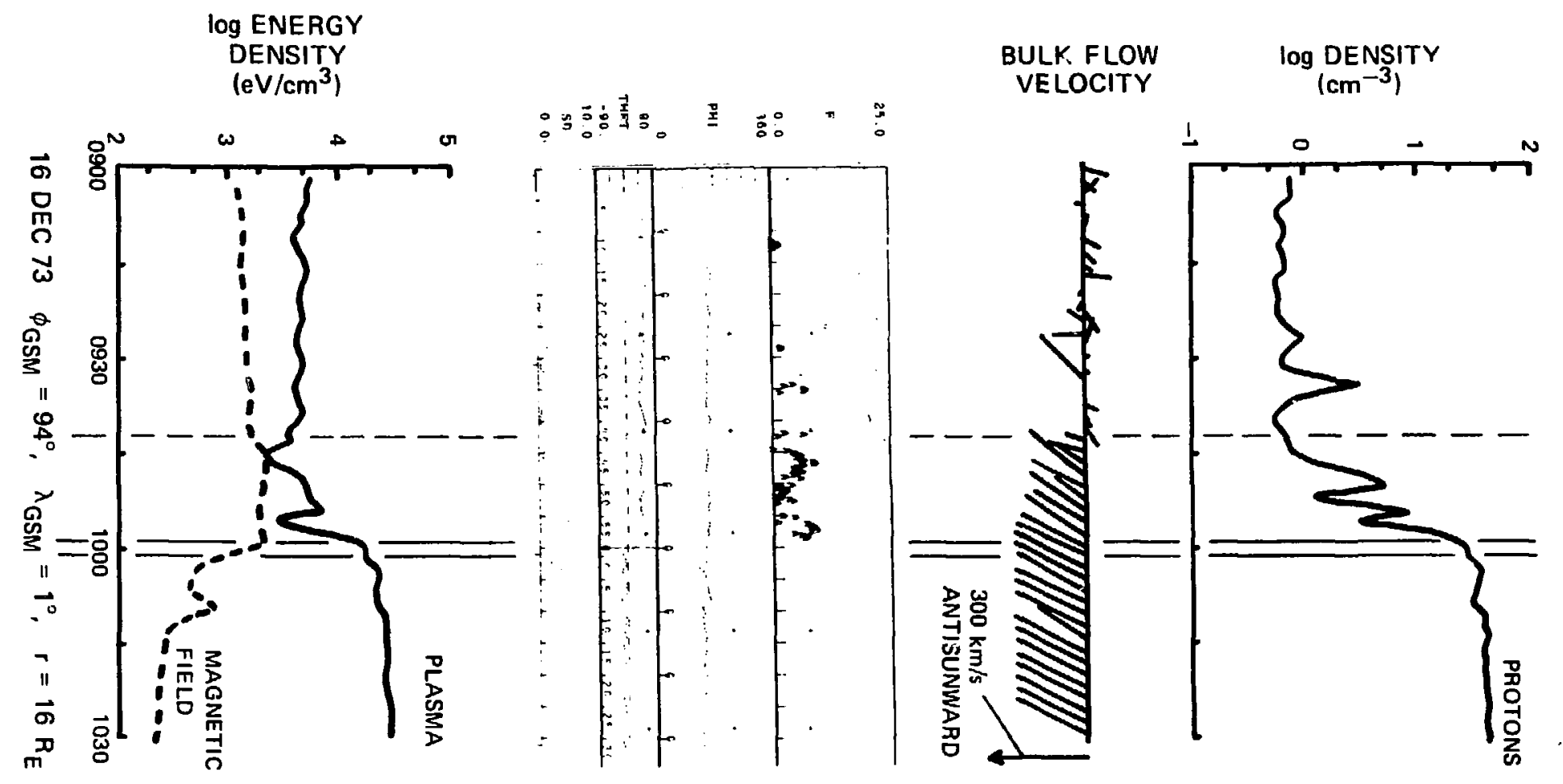



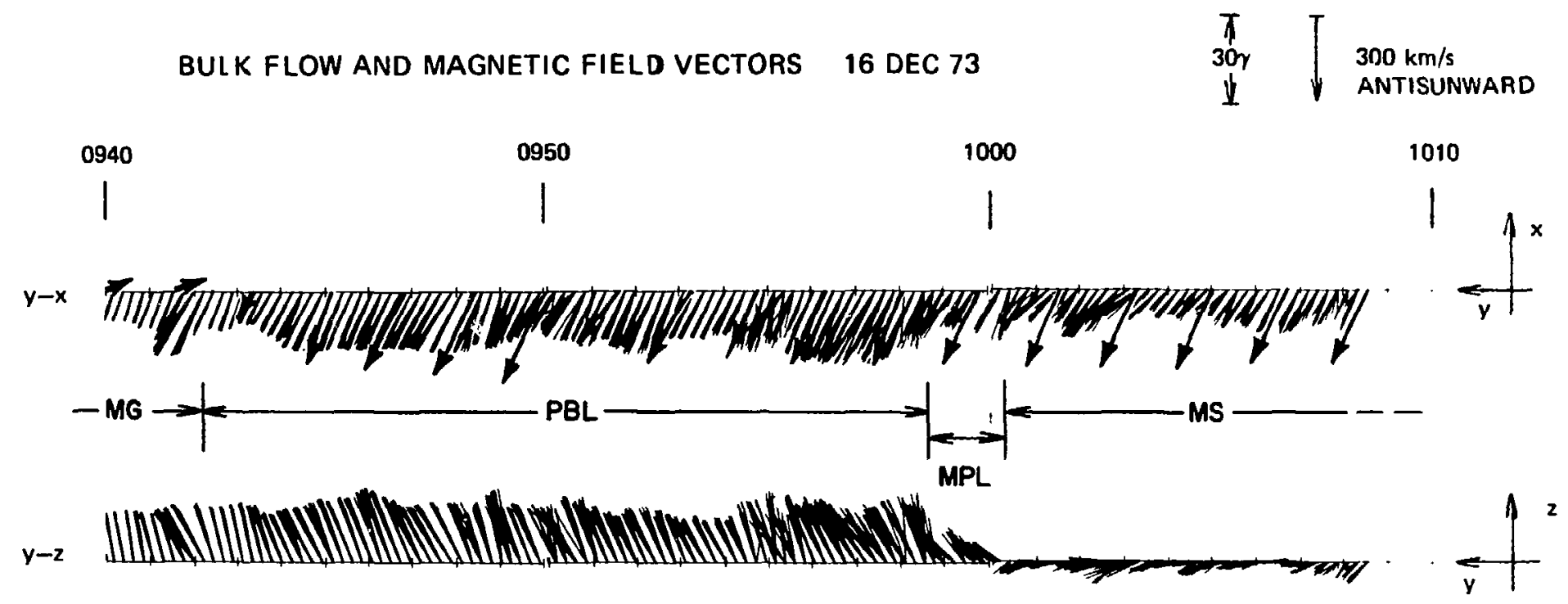


\section{APPENDIX C}

\section{MINIMUM VARIANCE ANALYSIS}

The minimum variance method was developed to provide a natural coordinate system for viewing field variations across a field discontinuity or current layer (Sonnerup and Cahill, Jr., 1967; Sonnerup and Ledley, 1974; Sonnerup, 1976). An orthogonal coordinate system is formed by the principal axes of the variance ellipsoid of the magnetic field data set sampled across the discontinuity. These principal axes are obtained by evaluating the eigenvector associated with the minimum eigenvalue of the matrix

$$
M_{i j}=\left\langle B_{i} B_{j}\right\rangle-\left\langle B_{i}\right\rangle\left\langle B_{j}\right\rangle
$$

where $\langle$ denotes an average of data points sampled across the discontinuity. This eigenvector represents a local estimate of the directio: normal to the magnetopause.

If we denote the largest, intermediate, and smallest eigenvalues of $M_{i j}$ with the subscripts 1,2 , and 3 , respectively, then the unit eigenvectors $\left(\hat{n}_{1}, \hat{n}_{2}, \hat{n}_{3}\right)$ can form an orthogonal right-hand coordinate system. In general, $\mathrm{n}_{3}$ (the normal component) is positive away from the earth, whereas $n_{1}$ and $n_{2}$ are directed roughly north and west, respectively, as shown in Figure $\mathrm{C} 1$.

The minimum variance technique assumes that
(1) the magnetopause structure is one dimensional,
(2) the magnetopause structure is stationary during the sampling period, and 


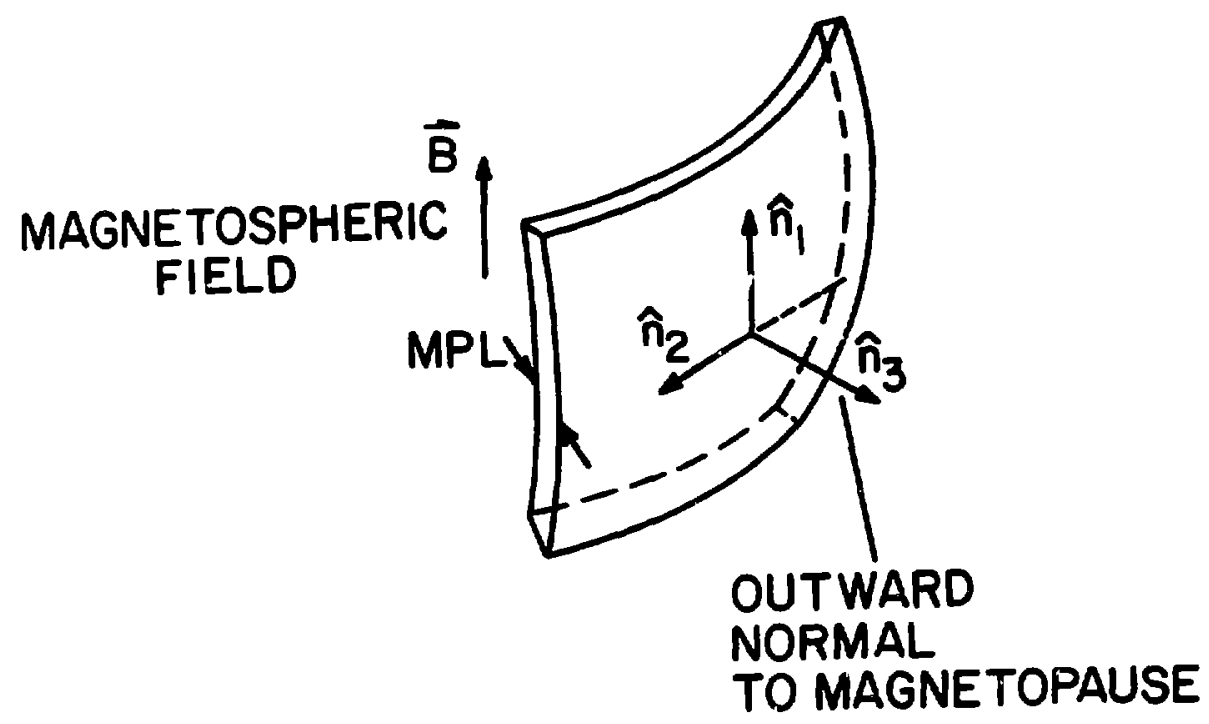

Figure $\mathrm{C} 1$ The coordinate system obtained by the minimum variance technique. 
(3) the current within the layer is not unidirectional (Sonnerup, 1975).

Magnetic field hodograms show magnetic field variations across the discontinuity displayed in the minimum variance coordinate system.

Field components $\left(B_{1}, B_{2}, B_{3}\right)$ are taken along the eigenvector directions $\left(\hat{\mathrm{B}}_{1}, \hat{\mathrm{n}}_{2}, \hat{\mathrm{n}}_{3}\right)$. A (B1, B2) plot displays variations of the field components tangent to the magnetopause and a (B1, B3) plot displays variations in a plane normal to the magnetopause.

The utility of magnetic field hodograms must be considered on a case-by-case basis. Waves traveling along the magnetopause have been inferred on the basis of variations between normal directions obtained for successive MPL crossings during multiple MPL crossings (e.g., Kaufmann and Cahill, 1977). The effects of MHD wave transmission and production near the magnetopause is superimposed on the general characteristics of the MPL-PBL region described in this thesis (see Wolf and Kaufmann, 1975). 


\section{REFERENCES}

Akasofu, S.-I., E. W. Hones, Jr., S. J. Bame, J. F. Asbridge, and A. T. Y. Lui, Magnetotail and boundary layer plasmas at geocentric distance of $18 \mathrm{R}_{\mathrm{E}}$ : Vela 5 and 6 observations, J. (6ephys. Res. 78 , $7257,1973$.

Axford, W. I. and C. O. Hines, A unifying theory of hi, n-latitude geophysical phenomena and geomagnetic storms, Can. J. Phys., 39, $1433,1961$.

Biermann, L., Kometenschweife und solare korpuskularstrahlung, $\mathrm{Z}$. Astrophys. 29, 274, 1951.

Cahill, L. J., Jr., and P. G. Amazeen, The boundary of the geomagnetic field, J. Geophys. Res., 68, 1835, 1963.

Caponi, M. Z, and N. A. Krall, Anomalous heat conduction along the field lines for turbulently heated plasmas, Phys. Fluids, 18, 699, 1975. Chapman, S., The motion of a neutral ionized stream in the earth's magnetic field, Proc. Cambridge Phil. Soc., 21, 577, 1923.

Chapman, S. and V. C. A. Ferraro, A new theory of magnetic storms, Terr. Mag. Atmos. Elect., 36, 77-97, 171-186, 1931.

Cole, K. D., Outline of a theory of solar wind interaction with the magnetosphere,Planet. Space Sci., 22, 1075, 1974.

Coleman, P. J., Jr., A model of the geomagnetic cavity, Radie Science, $6,321,1971$.

Crooker, N. U., Explorer 33 entry layer observations, J. Geophys. Res., $\underline{82}, 515,1977$.

Crooker, N. U., Dayside merging and cusp geometry, in press, J. Geophys. Res. , 1978. 
Crooker, N. U., T. E. Eastman and G. S. Stiles, Observations of plasma depletion in the magnetosheath at the dayside magnetopause, in press, J. Geophys. Res., 1978.

Davidson, R. C. and N. T. Gladd, Anomalous transport properties associated with the lower-hybrid-drift instability, Phys. Fluids, $18,1327,1975$.

Davidson, R. C., N. T. Gladd, C. S. Wu and J. D. Huba, Effects of finite plasma beta on the lower-hybrid-drift instability, Phys. Fluids, 20, 301,1977 .

Davidson, R. C., N. T. Gladd and Y. Goren, Influence of magnetic shear on the lower-hybrid-drift instability in toroidal reversed-field pinches, Phys. Fluids, 21, 992, 1978.

Dubinin, E. M., I. M. Podgorny and Yu. N. Potanin, Convection in the magnetosphere (laboratory experiment data), Academy of Sciences of the USSR Space Research Institute, Report No. 285, June, 1976.

Dungey, J. W., Interplanetary magnetic fields and the auroral zones, Phys. Rev. Letters, $6,47,1961$.

Eastman, T. E., E. W. Hones, Jr., S. J. Bame, and J. R. Asbridge, The magnetospheric boundary layer: Site of plasma, moment um and energy transfer from the magnetosheath into the magnetosphere," Geophys. Res. Lett., 3, 685, 1976.

Eastman, T.E. and E. W. Hones, Jr., Characteristics of the magnetospheric boundary laver and magnetopause layer as observed by IMP 6, in press, J. Geophys. Res., 1978. 
Eastman, T. E. and E. W. Hones, Jr., The magnetopause layer and plasma boundary layer of the magnetosphere, in press, Quantitative Modeling of Magnetospheric Processes, American Geophysical Union, Geophysical Monograph No. 21, June, 1979.

Eviatar, A, and R. A. Wolf, Transfer processes in the magnetopause, J. Geophys. Res., 73, 5561, 1968.

Fairfield, D. H., K. W. Behannon, R. P. Lepping, and N. F. Ness, Trajectories of Explorers 33, 35, 41, 43 and 47 May 1969 - December 1972, Goddard Space Flight Center report No. X-692-73-291, October, 1973.

Fairfield, D. H., Magnetic fields of the magnetosheath, Rev. Geophys. Space Phys., 14, 117, 1976.

Fairfield, D. H., Waves in the vicinity of the magnetopause, in Magnetospheric Particles and Fields, B. M. McCormack, ed., D. Reidel, Dordrect, Netherlands, 1976.

Fairfield, D. H., and E. W. Hones, Jr., IMP 6 measurements in the distant polar cusp during substorms, J. Geophys. Res., 83, 4273, 1978 .

Ferraro, V. C. A., On the theory of the first phase of a geomagnetic storm: A new illustration calculation based on an idealized (plane not cylindrical) model field distribution, J. Geophys. Res., 27, $155,1952$.

Forslund, D. W., J. M. Kindel, and M. A. Stroscio, Current driven electromagnetic ion cyclotron instability, submitted to J. Plasma Phys., Los Alamos Scientific Laboratory Report, LA-UR-78-780, March 1978. 
Freeman, J. W., Jr., C. S. Warren, and J. J. Maguire, Plasma flow directions at the magnetopause on January 13 and $14,1967, \mathrm{~J}$. Geophys. Res., 73, 5719, 1968.

Gary, S. P., R. A. Gerwin and D. H. Forslund, Electromagnetic current Instabilities, Phys. Fluids, 19, 579, 1976.

Gary, S. P. and J. J. Sanderson, Electrostatic temperature gradient drift instabilites, in press, Phys. Fluids, 1979.

Gary, S. P., Second order theory for electrostatic instabilities, submitted to Nuclear Fusion, Los Alamos Scientific Report, LA-UR-79-682, March, 1979.

Gurnett, D. A., R. R. Anderson, B. T. Tsuratani, E. J. Smith, G. Paschmann, G. Haerendel, S. J. Bame, and C. T. Russell, Plasma wave turbulence at the magnetopause: Observations from ISEE 1 and 2, submitted to J. Geophys. Res., February, 1979 (D. A. Gurnett, Department of Physics and Astronomy, University of Iowa, Iowa City, IA 52242).

Haerendel, G., Microscopic plasma processes related to reconnection, J. of Atm. Terr. Phys., 40, 343, 1978.

Haerendel, G., G. Paschmann, N. Sckopke, H. Rosenbauer, and P. C. Hedgecock, The frontside boundary layer of the magnetosphere and the problem of reconnection, J. Geophys. Res., 83, 3195, 1978a.

Haerendel, G., G. Paschmann, E. G. Shelley, and W. K. Peterson, On the origin of the magnetospheric boundary layer plasma, in press, $J$. Geophys. Res., 1978 b.

Hasegawa, A., K. Mima, Anomalous transport produced by kinetic Alfvén wave turbulence, J. Geophys. Res., 83, 1117, 1978. 
Hedgecock, P. C. and B. T. Thomas, HEOS observations of the configuration of the magnetosphere, Geophys. J. Roy. Astr. Scc., 41, $391,1975$.

Heikkila, W. J., Electric field topology near the dayside magnetopause, J. Geophys. Res., 8ㄱ, $1071,1978$.

Hess, W. N., The Radiation Belt and Magnetosphere, Blaisdell Publishing Co., Waltham, MA, USA, 1968.

Hill, T. W., Origin of the plasma sheet, Rev. Geophys. Space Phys., 12, $379,1974$.

Hill, T. W. and R. A. Wolf, Solar-wind interactions, in the The Upoer Atmosphere and Magnetosphere, National Academy of Sciences, 1977. Hones, E. W., Jr., J. R. Asbridge, S. J. Bame, M. D. Montgomery, S. Singer, and S.-I. Akasofu, Measurements of magnetotail plasma flcw made with VELA 4B, J. Geophys. Res., 77, 5503, 1972.

Howe, H. C., Jr., and J. H. Binsack, "Explorer 33 and 35 plasma observations of magnetosheath flow," J. Geophys. Res., 77, 3334, 1972.

Hudson, P. D., Rotational discontinuities in an anisotropic plasma, Planet. Space Sci., 19, 1693, 1971.

Ivanov, K. G., Magnetospheric surface flow with strong discontinuities, Astrophys. Space Sci., 31, 287, 1974.

Johnson, F. H., The driving force for magnetospheric convection, Rev. Geophys. Space Phys., 16, 161, 1978.

Kaufmann, R. L. and L. J. Cahill, Jr., The magnetopause at $5.2 R_{E}$ on August 4, 1972: Magnetopause shape and structure, J. Geophys. Res., 82, 1573, 1977. 
Kaufmann, R. L. and J. T. Horng, Physical structure of hydromagnetic disturbances in the inner magnetosheath, J. Geophys. Res., 76, 8189, 1971.

King, J. H., Interplanetary Medium Data Book - Appendix NSSDS/WDS-A-R\&S 77-04a, NASA/GSFC, September, 1977.

Krall, N. A. and A. W. Trivelpiece, Principles of Plasma Physics, McGraw-Hill, New York, 1973.

Landau, L, D. and E. M. Lifshitz, Electrodynamics of continuous media, Pergamon Press, Oxford, 1960.

Lemaire, J., Impulsive penetration of filamentary plasma elements into the magnetospheres of the Earth and Jupiter, Planet. Space Soi., 25, $887,1977$.

Lemaire, J., and M. Roth, Penetration of solar wind plasma elements into the magnetopshere, J. Atm. and Terr. Phys., 40, 331, 1978.

Lemaire, J., M. J. Rycroft and M. Roth, Control of impulsive penetration of solar wind irregularities into the magnetosphere by the interplanetary magnetic field direction, Aeronomica Acta, No. 193, Belgian Space Aeronomy Institute, 1978.

Lemaire, J., The magnetospheric boundary layer: A stopper region for a gusty solar wind, in press, Quantitative Modeiing of Magnetospheric Processes, American Geophysical Union, Geophysical Monograph No. 21, 1979.

Lemons. D. S. and S. P. Gary, Electromagnetic effects on the modified two-stream instability, J. Geophys. Res., 82, 2337, 1977.

Lemons, D. S. and S. P. Gary, Current-driven instabilities in a laminar perpendicular shock, J. Geophys, Res., 8 i3, 1625, 1978. 
Liewer, P. C. and R. C. Davidson, Sheath broadening by the lower-hybrid-drift instability in post-implosion theta pinches, Nuclear Fusion, 17, 85, 1977.

Levy, R. H., H. E. Petschek and G. I. Siscoe, Aerodynamic aspects of magnetospheric flow, AIAA J., 2, 2065, 1964.

Lindemann, F. A., Note on the theory of magnetic storms, Phil. Mag. 38 , $669,1919$.

Lynn, Y. M., Discontinuities in an anisotropic plasma, Phys. Fluids, 10, 2278,1967 .

Nishida, A., Geomagnetic diagnosis of the magnetosphere, SpringerVerlag, Berlin, 1978.

Palmer, I. D., and E. W. Hones, Jr., Characteristics of energetic electrons in the vicinity of the magnetospheric boundary layer at Vela orbit, J. Geophys. Res., 83, 2584, 1978.

Parker, E. N., Small-scale nonequilibrium of the magnetopause and its consequences, J. Geophys. Res., 72 4365, 1967.

Paschmann, G., G. Haerendel, N. Sckopke, H. Rosenbauer, and P. D. Hedgecock, "Plasma and magnetic field characteristics of the distance polar cusp near local noon: The entry layer," J. Geophys. Res., 81, 2883, 1976.

Paschmann, G., N. Sckopke, G. Haerendel, J. Papamastorakis, S. J. Bame, J. R. Asbridge, J. T. Gosling, E. W. Hones, Jr. and E. R. Tech, ISEE plasma observations near the subsolar magnetopause, in press, Space Science Reviews, July, 1978. 
Podgorny, I. M., E. M. Dubinin, Yu. N. Potanin, The magnetic field on the magnetopsheric boundary from laboratory simulation data, Geophys. Res. Lett., 4, 207, 1978a.

Podgorny, I. M., E. M. Dubinin, Yu. N. Potanin, New interpretation of changes in plasma parameters along trajectories of earth's satellites, Kosmicheskie Issledovaniya, 16, 522, 1978.

Roederer, J. G., Global problems in magnetospheric plasma physics and prospects for their solution, Space Science Reviews, 21, 23, 1977. Rosenbauer, H., H. Grunwaldt, M. D. Montgomery, G. Paschmann, and N. Sckopke, HEOS 2 plasma observations in the distant polar magnetosphere: The plasma mantle, J. Geophys. Res., 뭉, 2723, 1975.

Rossi, B. and S. Olbert, Introduction to the physics of space, McGraw Hill, New York, 1970.

Russell, C. T. and R. C. Elphic, Initial ISEE magnetometer results: Magnetopause observations, in press, Space Science Reviews, August, 1978.

Scarf, F. L., L. A. Frank, and R. P. Lepping, Magnetosphere boundary observations along the IMP 7 orbit 1 , boundary locations and wave Jevel variations, J. Geophys. Res., 르, 5171, 1977.

Schindler, $K_{.}$, On the role of irregularities in plasma entry into the magnetosphere, submitted to J. Geophys. Res., Los Alamos Scientific Laboratory Report, LA-UR-97-456, February 1979.

Sckopke, N., G. Paschmann, H. Rosenbauer and D. H. Fairfield, Influence of the interplanetary magnetic field on the occurrence and thickness of the plasma mantle, J. Geophys. Res., 81, 2687, 1976. 
Sckopke, N. and G. Paschmann, The plasma mantle: A survey of magnetotail boundary layer observations, J. of Atm. and Terr. Phys., 40, 261, 1978.

Siscoe, G. L., L. Davis, Jr., P. J. Coleman, Jr., E. J. Smich and D. E. Jones, Power spectra and discontinuities of the interplanetary magnetic field: Mariner 4, J. Geophys. Res., 73, 61, 1968.

Sonnerup, B. U. Ö. and B. G. Ledley, Magnetopause rotational forms, J. Geophys. Res., 79, 4309, 1974.

Sonnerup, B. U. Ö., Magnetopause and boundary layer, in Physics of solar Planetary Environments Vol. II, D. J. Williams, ed., American Geophysical Union, 1976.

Sonnerup, B. U. Ö. and L. J. Cahill, Jr., Magnetopause structure and altitude from Explorer 12 observations, J. Geophys. Res., 72, 171, 1967.

Sonnerup, B. U. Ö., Magnetopause structure, paper presented at the ISEE Workshop at the Space Sciences Laboratory, University of California, Berkeley, December, 1977 (B. U. O. Sonnerup, Radiophysics Laboratory, Dartmouth College, Hanover, NH 03755).

Sonnerup, B. U. Ö., Transport mechanisms at the magnetopause, invited paper presented at the AGU Chapman Conference on Magnetospheric Substorms and Related Plasma Processes, Los Alamos, NM, October, 1978.

Spitzer, L., Jr., Phusics of fully ionized gases, Interscience, New York, 1962.

Stiles, G. S., Notes on the IMP magnetotail plasma analysis, Los Alamos Scientific Laboratory internal report, August, 1975 (E. W. Hones, 
Jr., University of California, Los Alamos Scientific Laboratory, P. 0. Box 1663, Los Alamos, NM 87545).

Stiles, G. S., E. W. Hones, Jr., S. J. Bame, and J. R. Asbridge, Plasma sheet pressure anisotropies, J. Geophys. Res., 83, 3166, 1978.

Theodoridis, G. C. and F. R. Paolini, The angular response of spherical plate electrostatic analyzers, Rev. Sci. Instrum., 4Q, 621, 1969. Vasyliunas, V. M., Deep space plasma measurements, in Methods of Experimental Physies, Vol. IX, edited by H. R. Grien and R. H. Lovberg, Academic Press, New York, 1969.

Vasyliunas, V. M., Theoretical models of magnetic field line merging, 1 , Rev. Geophys. Space Phys., 13, 303, 1975. West, H. I., Jr., R. M. Buck, and J, R. Walton, Electron pitch angle distributions throughout the magnetosphere as observed on 0G0-5, J. Geophys. Res., 78, 1054, 1973.

Williams, D. J., Magnetopause characteristics inferred from threedimensional energetic particle distributions, submitted to $\mathrm{J}$. Geophys. Res., June, 1978 (D. J. Williams, NOAA/ERL/Space Environment Laboratory, Boulder, CO 80302). Willis, D. M., Structure of the magnetopause, Rev. Geophys. Sp. Phys., 2, 953,1971 .

Wolfe, J. H., R. W. Silva, and M. A. Myers, Obserations of the solar wind during the flight of IMP 1, J. Geophys. Res., 71, 1319, 1966. Wolfe, A. and R. L. Kaufmann, MHD wave transmission and production near the magnetopause, J. Geophys. Res., 80, 1764, 1975.

Yang, C.-K. and B. U. Ö. Sonnerup, Compressible magnetopause reconnection, J. Geophys. Res., 82, 299, 1977. 